\title{
TOWARDS SYSTEMATIC CONTROLLER DESIGN IN REHABILITATION ROBOTS
}

ZHANG JUANJUAN

School of Electrical and Electronic Engineering

\begin{abstract}
A thesis submitted to the Nanyang Technological University in partial fulfillment of the requirements for the degree of Doctor of Philosophy
\end{abstract}





\section{Acknowledgments}

First of all, I would like to express my sincere gratitude to my advisor Prof. Cheah Chien Chern and my co-advisor Prof. Steven H. Collins for their continuous guidance and motivation that have boosted my academic and personal growth, and the patience they have extended on me along the way.

I thank Prof. Hartmut Geyer and his group for kindly sharing their laboratory space and equipment.

I thank my fellow colleagues in the Intelligent Robotics Lab of Nanyang Technological University and Experimental Biomechatronics Lab of Carnegie Mellon University for the assistance they have given to my work, the stimulating discussions we had together and the personal caring they have given to me in time of difficulties.

I thank my friends who have shared my joys and sorrows during my $\mathrm{PhD}$ studies, the support of whom has helped me go through a long journey.

I thank the Singapore Economic Development Board and National Science Foundation of the U.S. and for funding my research.

Finally, I would like to extend my gratitude to my family. I thank my parents and 

my sister for supporting me spiritually. I especially thank my husband, Heming Song, for his tremendous patience, continuous support and wonderful love that have given me the strength of conquering various difficulties during my studies. 



\section{Contents}

ACKNOWLEDGMENTS i

$\begin{array}{lll}\text { ABSTRACT } & \text { ix }\end{array}$

LIST OF TABLES Xiv

LIST OF FIGURES $\quad$ xxvi

1 Introduction 1

1.1 Motivation ...................... 1

1.2 Upper-Limb Rehabilitation Robots Control Challenges . . . . . . . . . 2

1.3 Lower-Limb Rehabilitation and Assistive Robots Control Challenges . . 3

1.4 Main Contributions . . . . . . . . . . . . . . . . . . . 4

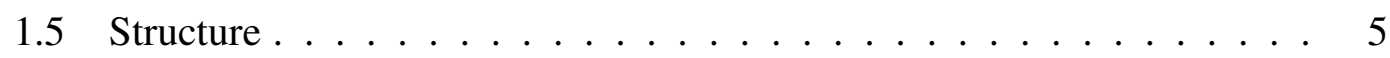

2 Background 9 
2.1 Introduction . . . . . . . . . . . . . . . 9

2.2 Motor Control Rehabilitation . . . . . . . . . . . . . . . . . . 9

2.2.1 Upper-Limb Rehabilitation Robots . . . . . . . . . . . . . . 11

2.2.2 Lower-limb Rehabilitation Robots . . . . . . . . . . . . . 11

2.3 Interaction Control . . . . . . . . . . . . . . . 11

2.3.1 Rehabilitation Robot Control . . . . . . . . . . . . . . 13

2.3.2 Low-level Physical Human-Robot Interaction Control . . . . . 14

2.4 Key Control Algorithms _. . . . . . . . . . . . . . . 15

2.4.1 Passivity-based Control . . . . . . . . . . . . . 15

2.4 .2 Iterative learning $\ldots \ldots \ldots \ldots$

2.4.3 Covariance Matrix Adaptation Evolution Strategy . . . . . . . . 17

3 Upper-limb Rehabilitation Robots Control

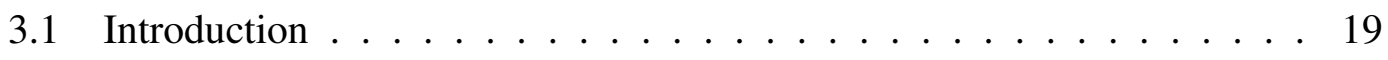

3.2 Upper-limb Rehabilitative Robotics Dynamics . . . . . . . . . . . . . . 22

3.3 Human-Robot Interaction Involved Control . . . . . . . . . . . . 23

3.3.1 Motion-related Controller . . . . . . . . . . . 26

3.3.2 Interaction-related Controller . . . . . . . . . . . 35

3.4 Passivity and Stability of the System . . . . . . . . . . . . . . . 39 
3.4.1 System Passivity . . . . . . . . . . . . . . . . 39

3.4 .2 Convergence .................... . . 43

3.5 Simulation and Experiments . . . . . . . . . . . . . 46

3.5 .1 Simulation .................... 47

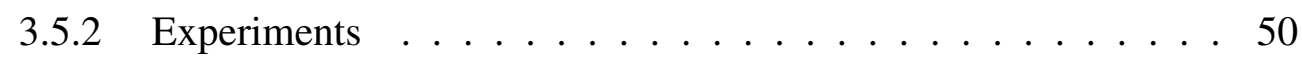

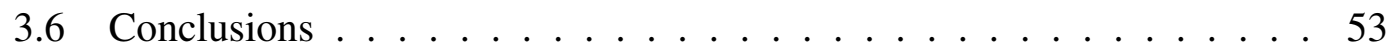

4 Lower-limb Exoskeletons Torque Control 55

4.1 Introduction . . . . . . . . . . . . . . 55

4.2 Methods ............................. 62

4.2.1 Exoskeleton system . . . . . . . . . . . . . . . . 62

4.2 .2 System Modeling . . . . . . . . . . . . . . . . . . . 64

4.2 .3 Control Issues $\ldots \ldots 67$

4.2 .4 Controllers ................... 70

4.2.5 Experimental Methods . . . . . . . . . . . . 88

4.3 Results.............................. 90

4.4 Discussion . . . . . . . . . . . . . . . . . . 92

4.4.1 Proportional-learning-damping control ... . . . . . . 94

4.4.2 Benefits of additional control elements . . . . . . . . . . 98 
4.4 .3 Factors Limiting Interpretation . . . . . . . . . . . . . . . 102

4.4.4 Implications for control of future systems . . . . . . . . . . 105

4.5 Conclusions . . . . . . . . . . . . . . . . . . 105

4.6 Supporting Materials . . . . . . . . . . . . . . 106

5 Iterative Learning Gain Optimization $\quad 117$

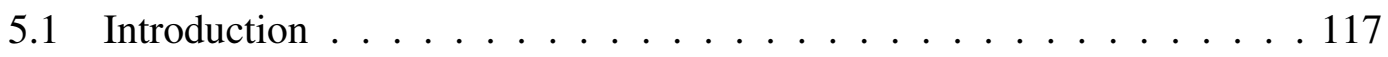

5.2 Methods ............................. 119

5.2.1 Simplified System Model . . . . . . . . . . . . . . . . . . 120

5.2 .2 Controllers . . . . . . . . . . . . . . . 122

5.2.3 Theoretical Analysis on Optimal Iterative Learning Gain . . . . 124

5.2.4 Testbed System Configurations . . . . . . . . . . . . . . . . 128

5.2.5 Experimental Methods . . . . . . . . . . . . . . . 129

5.3 Results . . . . . . . . . . . . . . . . . . . . . . 134

5.4 Discussion . . . . . . . . . . . . . . . . . . 136

5.5 Conclusions . . . . . . . . . . . . . . . . 137

6 Passive Series Stiffness Optimization 139

6.1 Introduction . . . . . . . . . . . . . . . . . . . 139 


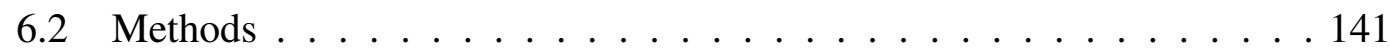

6.2.1 Exoskeleton system and simplified model . . . . . . . . . . . 142

6.2 .2 Controllers . . . . . . . . . . . . . . . . . 142

6.2.3 Theoretical Analysis and Hypotheses . . . . . . . . . . . . . 144

6.2.4 Dynamic Complications not Featured . . . . . . . . . . 155

6.2.5 Testbed System Configurations . . . . . . . . . . . . . 156

6.2.6 Experimental Methods . . . . . . . . . . . . 156

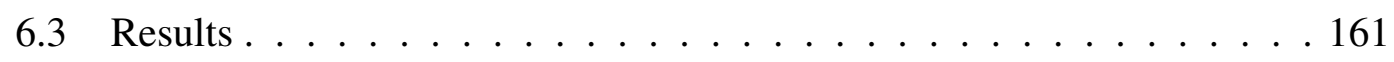

6.4 Discussion . . . . . . . . . . . . . . . . . . 164

6.5 Conclusions . . . . . . . . . . . . . . . . . . 168

6.6 Acknowledgment . . . . . . . . . . . . . . . . . 169

7 High Level Control Online Optimization $\quad 171$

7.1 Introduction . . . . . . . . . . . . . . . 171

7.2 Methods . . . . . . . . . . . . . . . . 173

7.2.1 Exoskeleton Testbed . . . . . . . . . . . . 173

7.2.2 Parameters to Be Optimized: High Level Controller . . . . . . . 176

7.2.3 Low Level Torque Controller: Proportional Control + Damping Injection + Iterative Learning $\ldots$. . . . . . . . . 177 
7.2.4 Estimation of Metabolic Rates from Respiratory Measurements 178

7.2.5 Optimization Algorithms . . . . . . . . . . . . . . . 180

7.2.6 Experimental Methods . . . . . . . . . . . . . . . 181

7.3 Results . . . . . . . . . . . . . . . . . 183

7.4 Discussion . . . . . . . . . . . . . . . . . . . . . . 184

8 Conclusions and Future Work 189

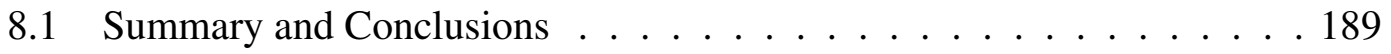

8.2 Future Work . . . . . . . . . . . . . . . . . . . . . . . 194

APPENDIX A . . . . . . . . . . . . . . . . 197

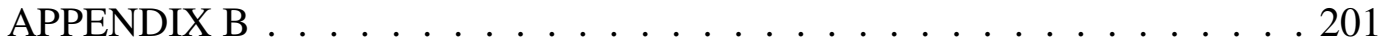

APPENDIX C . . . . . . . . . . . . . . . . . . 204

PUBLICATIONS 


\section{Abstract}

Robotic devices have been used in post-stroke or trauma neurorehabilitation process for years. Due to the uncertain, complex, and changing physical human-robot-interaction dynamics involved and task-specific objectives for rehabilitation, control of this type of devices remains a vast area of research. This thesis seeks systematicness in rehabilitation robot control design by developing control frameworks for upper- and low-limb rehabilitation devices that can be generally applied on multiple devices, using theoretical and experimental methodologies.

The thesis examines the control difficulties and provides solutions for two specific groups of rehabilitation robots that are widely used: the upper-limb rehabilitation robot for reaching and tracking tasks that consists of a robot arm linked to human arm in series and the powered ankle exoskeleton for human gait training. To address systematic control for these two groups of robots, the thesis investigates several separate problems.

The stability of human-robot interaction in upper-limb rehabilitation robots for reaching and tracking tasks training is solved in this thesis in a systematic way by means of nonlinear control theory. In addition to stability, the control framework developed ensures human safety and realizes different operations modes during training: humandominant mode in which human is partially paralyzed and given relative larger freedom to exercise to enhance mobility, robot-dominant mode in which human movements are less reliable and more robot assistance is needed and system-stop mode in which the ac- 
tual human movements drift too far from the desired trajectories and should be stopped to avoid human injuries. This passivity-based, provably stable control framework can be extended to various upper-limb rehabilitation devices as a control base. Functionality of the controller was tested and confirmed effective through computer simulation and hardware experiments.

This thesis also develops a robust torque control structure for tethered ankle orthoses in human walking driven by series elastic actuators in face of the complicated and time-varying system dynamics. Major challenges of this control problem lie in the complicated and changing human-robot interactive dynamics, state-dependent rapidly varying torque objectives, and complex friction dynamics in Bowden cable transmission. This thesis designs, implements and tests a series of prominent torque controllers for powered lower-limb exoskeleton during human walking, including the combination and variation of model-free, model-based, feedback and feed-forward controllers. According to the experimental results, model-based control elements have little or even negative benefits on control performance due to the changing dynamics while requiring great efforts on system identification and high computation power. Continuous integral actions are not effective either due to the nonlinear and changing system dynamics. It is shown that the combination of model-free, integration-free feedback control and iterative learning is best suited for this type of devices used in cyclic applications like locomotion. The results support this controller in various robotic systems with properties similar to ankle exoskeletons such as nonlinear, complicated and changing dynamics, and cyclic operations, e.g., legged robots, lower-limb prostheses.

With suitable torque control architecture identified for ankle exoskeletons during human walking, this thesis also investigates by theory and experiments the interactions of actuator compliance, desired torque profile and control gains for the sake of further improving the real-time torque tracking performance. The theoretical analysis and large amount of experimental results show that for a desired quasi-stiffness generated by re- 
lating torque and ankle angle, the optimal actuator passive stiffness for control is the one that matches the desired one. For a desired passive actuator stiffness, the optimal iterative learning gain that benefits real-time tracking performance matches the inverse of passive stiffness. These results provide guidance for ankle exoskeleton system elasticity with a fixed control objective, and for iterative controller gain with a combination of fixed control objective and system elasticity.

With low-level torque control solved at a satisfactory extent, this thesis also develops an online optimization system for multivariate high level controllers in lower-limb exoskeletons from human metabolic measurements. This system enables fast and customized automation of the identification of assistance conditions that benefit human energy efficiency. Four searching algorithms, including both gradient-based and nongradient methods, were tested in simulation. Two of them, Nelder-Mead method, and Covariance Matrix Adaptation Evolution Strategy, were selected for hardware implementation due to their suitability for high dimension, high noise searching problems revealed by simulations. This system was implemented and tested on an ankle exoskeleton. Experiments demonstrated the effectiveness of both methods, especially CMA-ES, on the optimization of multi-parameter high level controller using real-time human respiratory measurements. Total evaluation time used to optimize multivariate high level controllers in human-robot interactions is greatly reduced while human metabolic rate is reduced significantly. The system can be easily extended to other human-robot interactive systems that aim for human metabolic benefits, and can be scaled to higher dimensional parameter optimization without heavily increasing evaluation time. This on-line optimization process is expected to be conducted at the beginning of each long experimental or training sessions for all subjects, involvement of which is expected to improve the corresponding operation efficiency.

In summary, this thesis systematically solved 
$\diamond$ the stability and automated training provision of upper-limb rehabilitation robots,

$\diamond$ the robust and accurate control of robotic legged locomotion systems such as lower-limb exoskeletons, prosthesis and walking robots with the development of a robust control architecture, the optimization of control gains, and the optimization of series passive stiffness in actuation,

$\diamond$ and the automation of fast and customized identification of optimal energy efficient multivariate assistance conditions in human-robot interactive systems with an online optimization system using CMA-ES and Nelder-Mead. 


\section{List of Tables}

3.1 RMS values for region error, $e_{r}=\max \left(\|\Delta x\|-a_{h}, 0\right)$, and velocity tracking error, which is defined as $e_{v}=\left\|\dot{x}-\dot{x}_{d e s}\right\|$, in 2D experiments. . 51

4.1 Angle-based control parameter values . . . . . . . . . . . . 85

4.2 NMM parameter values . . . . . . . . . . . . . . . . . 87

4.3 EMG parameter values . . . . . . . . . . . . . . . . . . 87

4.4 Low-level torque control parameter values (Note: $I_{3}$ denotes a $3 \times 3$ identity matrix $\ldots \ldots \ldots \ldots \ldots \ldots \ldots$

4.5 Tracking errors with $\mathrm{PD}^{*}+\Delta \mathrm{LRN}$ torque control . . . . . . . . . . . . 92

5.1 List of desired stiffness tested in experiments with assigned ID . . . . . 130

5.2 List of springs used in experiments with assigned ID . . . . . . . 131

5.3 List of passive stiffness values . . . . . . . . . . . . . . . 134

6.1 Linear unit curve parameter values . . . . . . . . . . . . . . . 157 
6.2 Piece-wise linear unit curve parameter values . . . . . . . . . . . 157

6.3 List of springs used in experiments with assigned ID . . . . . . 158

6.4 List of measured stabilized passive stiffness values . . . . . . . . . . 162

1 Muscle model parameter values used in NMM . . . . . . . . . . . 203 


\section{List of Figures}

3.1 Operation regions that move along the desired trajectory $x_{d e s}(t)$ determined by real-time position error $\|\Delta x\|$. From inside to outside, there are three main regions, namely human-dominant region (H-DR, $\left.\|\Delta x\| \in\left[0, a_{h}\right]\right)$, robot-dominant region (R-DR, $\left.\|\Delta x\| \in\left(a_{h}, a_{r}\right]\right)$ and safety-stop region (S-SR, $\left.\|\Delta x\| \in\left(a_{r},+\infty\right)\right)$. The increasing (IR-DR, $\left.\|\Delta x\| \in\left(a_{h}, a_{i}\right]\right)$ and decreasing (DR-DR, $\left.\|\Delta x\| \in\left(a_{i}, a_{r}\right]\right)$ sub-regions of R-DR are identified by increasing and decreasing stiffness (Section III.A. $\dagger 2$ ). The transitional sub-region of S-SR (TS-SR, $\|\Delta x\| \in$ $\left.\left(a_{r}, a_{t}\right]\right)$ exists to ensure smooth transition from R-DR to stable S-SR (SS-SR, $\|\Delta x\| \in\left(a_{t},+\infty\right)$. See Section III.A. $\left.\dagger 1\right) . \ldots \ldots \ldots$

3.2 An illustration of the weight factor $w$ versus $\Delta x$ for $m=2$, with $a_{r}=$ 2, $a_{t}=4.5 . w=1$ when $\|\Delta x\| \in\left[0, a_{r}\right]$ in the human-dominant region (H-DR) and robot-dominant region (R-DR); $w=0$ when $\|\Delta x\| \epsilon$ $\left(a_{t},+\infty\right)$ in the stable safety-stop region (SS-SR). $w$ smoothly transits from 1 to 0 when $\|\Delta x\| \in\left(a_{r}, a_{t}\right]$ in the transitional safety-stop region (TS-SR). 
3.3 An illustration of the position-dependent stiffness $k_{p}$ versus $\delta x$ for $m=2$, with $a_{h}=1, a=0.3, k_{1}=2$. (a): A three-dimensional view; (b): A two-dimensional view: intersection of $k_{p}$ and surface $\delta x_{2}=0$. When $\|\delta x\| \in\left[0, a_{h}\right] \cup\left(a_{r},+\infty\right)$ in human-dominant region (H-DR) and safety-stop region (S-SR), $k_{p}=0$; When $\|\delta x\| \in\left(a_{h}, a_{r}\right]$ in robotdominant region (R-DR), $k_{p}>0$. Increasing robot-dominant region (IR-DR) denotes the sub-region of R-DR in which $\partial k_{p} / \partial\|\delta x\|>0$, i.e., $k_{p}$ increases as $\|\delta x\|$ increases. Decreasing robot-dominant region (DR-DR) denotes the sub-region with $\partial k_{p} / \partial\|\delta x\|<0 . \ldots 33$

3.4 Interaction force handling regions determined by the magnitude of directional difference, $\gamma$, between interaction force $f_{h}$ and the opposite of task space sliding vector $s_{x}$. When $\gamma \leq \beta, \beta \in[0, \pi / 2]$, system is in Retaining Region (RR); when $\gamma \in(\beta, \beta+\sigma], \sigma \in[0, \pi / 2]$, system is in Projecting Region (PR); otherwise, system is in Canceling Region (CR). 37

3.5 An illustration of the pseudo-energy versus the weighted position error $\delta x$ for $m=2: a_{h}=1, a=0.3, k_{1}=2$. For $\|\delta x\| \in\left[0, a_{h}\right]$, i.e., humandominant region, $P_{t}(\delta x)=0$; For $\|\delta x\| \in\left(a_{r},+\infty\right)$, i.e., safety-stop region, $P_{t}(\delta x)$ is saturated. For $\|\delta x\| \in\left(a_{h}, a_{r}\right]$ in the robot-dominant region, $P_{t}(\delta x)$ smoothly changes from 0 to its maximum. . . . . . . . 42 
3.6 Three-dimensional RRR-robot simulation results. (a): The desired and simulated trajectories in space; (b): The desired and simulated position trajectories of all three axes in time domain; (c): The instant distance of robot end-effector and desired central trajectory $\Delta x$ in time. From (a) and (b), it is seen that the system end-effector started at a location far away from the initial desired position $x_{d e s}(0)$ by design, which resulted in controller running safety-stop (S-S) mode at the beginning. As the desired trajectory $x_{d e s}$ approached the end-effector later (intentionally by trajectory planning), controller entered robot-dominant (R-D) mode at $t_{r}$. At $t_{h}$, the system first entered human-dominant region and started H-D Mode. R-D mode occasionally was still activated in this period to ensure that the system stayed in human-dominant region. . . . . . . . . 46

3.7 Experimental testbed: An upper-limb rehabilitative robotics composed of a 2-DOF planar robot, force sensor and a 2-DOF passive arm support. 48

3.8 Experimental results for assistive performance test, i.e., $a_{h}=0$. (a): Desired and actual trajectories in space; (b): Position tracking errors of both $x$ and $y$ directions in time domain; (c): Desired and actual velocities of both directions in time domain, in which $v_{1}$ denotes velocity on $x_{1}$ axis and $v_{2}$ is velocity on $x_{2}$ axis. . . . . . . . . .

3.9 Experimental results for rehabilitative performance test with $a_{h}=0.05 \mathrm{~m}$. (a): Desired and actual trajectories in space; (b): Region errors $e_{r}=$ $\max \left(\|\Delta x\|-a_{h}, 0\right)$ versus time; (c): Desired and actual velocities in time domain.

3.10 Experimental results for safety-stop performance test with $a_{h}=0.05 \mathrm{~m}$ and $a_{r}=0.0703 \mathrm{~m}$. (a): The force spike applied at the handle by the subject in time domain; (b): The desired and actual spatial trajectories. . 51 
4.1 Experimental Testbed: A) High speed stand-alone real-time controller reads sensory information, computes desired torques using high-level controller, computes desired motor velocity using low level controller, and outputs desired motor velocity to the motor drive. B) Dedicated motor drive. C) Powerful off-board geared motor and pulley. D) Bowden cable transmission. E) Lightweight instrumented ankle exoskeleton. F) Enlarged schematic of the exoskeleton. G) Photograph of the exoskeleton. . . . . . . . . . . . . . . . . . 60

4.2 Flowchart of the control system. High Level Control and Low Level Control are the two blocks to be varied in this study. . . . . . . . . . . 64

4.3 Complex torque versus exoskeleton ankle joint angle relationship when motor position was fixed and the exoskeleton was passively flexed for one hundred strides. . . . . . . . . . . . . . . . . . . 68

4.4 Variability in exoskeleton ankle joint angle trajectory during one hundred strides of walking with the motor fixed and the exoskeleton passively flexing. . . . . . . . . . . . . . . . . . . . 69

4.5 High-level control based on a trajectory in time . . . . . . . . . 84

4.6 High-level control based on ankle joint angle . . . . . . . . . . 85

4.7 Neuromuscular model control schematic . . . . . . . . . . . . . . . 86

4.8 Proportional electromyography control schematic . . . . . . . . . . 87

4.9 Root-mean-squared torque error calculated for all strides (RMS-E) and for an average stride (RMS-E AVG) across all high- and low-level control combinations. . . . . . . . . . . . . . . . . 91 
4.10 Time trajectories of desired torque (pink dots) and measured torque (gray dots) for 100 strides of walking, and average-stride desired torque (red line) and measured torque (gray line), for all combinations of con-

4.11 The contributions of each component of the $\mathrm{PD}^{*}+\Delta \mathrm{LRN}$ controller at steps 1-10, steps 51-60 and steps 101-110. In the first step proportional control dominates, and at steady state the learned component dominates. Data shown are from the Time-based high-level controller. Plots for all high-level conditions are available as Supplementary Materials (Fig. S5).

4.12 A block diagram of controller $L 9, \mathrm{PD}^{*}+\Delta \mathrm{LRN} \ldots \ldots 96$

5.1 Tethered ankle exoskeleton system. (A) A schematic of the system that consists of an off-board motor system, a Bowden cable transmission ended with a series spring and an exoskeleton frame. For each desired quasi-stiffness, passive stiffness of the system was varied by switching the series spring and walking experiments were conducted to identify the optimal passive stiffness value. (B) A photo of the device worn by a subject standing on treadmill. . . . . . . . . . . . . . . . . 12

5.2 The ankle angle based high-level desired torque curve imposed in experiments to realize different desired quasi-stiffness profiles. It commands desired torque that is linearly proportional to exoskeleton joint angle $\theta_{e}$ defined by anchor point $\left[\theta_{e, 0} 0\right]$ and desired quasi-stiffness

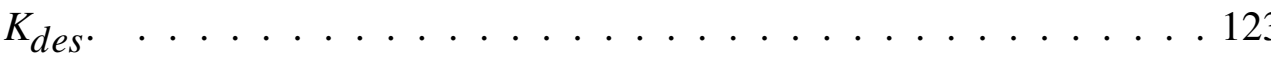


5.3 All three tested linear desired torque versus ankle angle curves used in the form of Eq. (6.2) with $\theta_{e, 0}=-2(\mathrm{deg})$ and $K_{d e s}$ values listed in Table 5.1. . . . . . . . . . . . . . . . . . . 130

5.4 Instantaneous passive stiffness values of passive walking sessions are plotted against the measured torques for various spring configurations, one session for each. One experiment session consists of one-hundred steps with motor position fixed. The stablized passive stiffness value for one session is defined as the median of the values over a stabilized region. The effective passive stiffness of the one stiffness configuration is defined as the mean of stabilized stiffness values across multiple sessions. . . . . . . . . . . . . . . . . . . 132

5.5 Centering process of index-wise ankle positions. a): Ankle position array of the one hundred steps investigated for an example time stamp within steps. b): Ankle position array as shown in a) zero-phase filtered with a 1/20 cut-off frequency butter-worth filter. c): Centered ankle position array achieved by subtracting array in b) from that in a). . . 133

5.6 Mean of step-wise root-mean-squared torque tracking errors of all combinations of learning gain, desired stiffness and passive stiffness and the relative errors to their peak desired torques are plotted against the natural logarithms of the learning relative to the predicted optimum $\ln \left(K_{l} / K_{l, o p t}\right.$. A: Values computed with raw torque errors. B: Values computed by normalizing raw torque errors by position-wise ankle position variance. . . . . . . . . . . . . . . . . . . 135 
6.1 The two types of ankle angle based high-level desired torque curves imposed in experiments to realize different desired quasi-stiffness profiles. (A) High-level controller I commands desired torque that is linearly proportional to exoskeleton joint angle $\theta_{e}$ defined by anchor point $\left[\begin{array}{lll}\theta_{0, l} & \tau_{0, l}\end{array}\right]$ and desired quasi-stiffness $K_{d e s}$. (B) High-level controller II commands desired torque that is piece-wise linearly proportional to joint angle $\theta_{e}$ with four phases defined by anchor points $\left[\theta_{0, p} \tau_{0, p}\right]$, $\left[\theta_{1, p} \tau_{1, p}\right],\left[\theta_{2, p} \tau_{2, p}\right],\left[\theta_{3, p} \tau_{3, p}\right]$ and $\left[\theta_{4, p} \tau_{4, p}\right]$. In both cases, desired torque is lower-bounded at zero. . . . . . . . . . . . . . . . 143

6.2 All eight tested desired torque versus ankle angle curves used to realize different desired quasi-stiffness values. (A) Three linear curves achieved by scaling unit curve defined by Table 6.1 with scaling factors of $0.4,1$ and 1.7. (B) Five piece-wise linear curves by achieved by scaling unit curve defined by Table 6.2 with scaling factors of $0.4,0.7$,

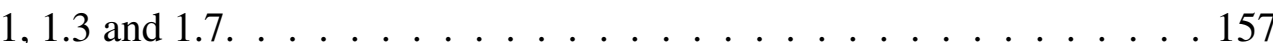

6.3 Instantaneous passive stiffness values of passive walking sessions are plotted against the measured torques for various spring configurations, one session for each. One experiment session consists of one-hundred steps with motor position fixed. The stablized passive stiffness value for one session is defined as the median of the values over a stabilized region. The effective passive stiffness of the one stiffness configuration is defined as the mean of stabilized stiffness values across multiple sessions. . . . . . . . . . . . . . . . . . 158 
6.4 Demonstration of the experimental procedure for one example combination of desired and passive stiffness. For stiffness combination, the first experiment session starts with a moderate set of proportional and damping injection gains. For the subsequent sessions, each of which includes at least one-hundred steady strides, the proportional gain was firstly increased until oscillations became noticeable. Then, damping injection gain was increased until oscillations were reduced to be imperceptible. Proportional and damping injection were then increased alternatively until perceptible oscillations seen with maximum damping injection gain. The root-mean-squared torque tracking errors of each session was then calculated and compared against one another. The best-performed session was identified with the lowest error and its tracking error and control gains are then registered as the estimates of optimal tracking performance and optimal control gains for this specific stiffness combination. The level of oscillation for each session is also displayed. It is seen that with the increase of control gains, torque tracking errors first drop, but later increase due to increasing oscillations. 160

6.5 Demonstration of signal level of oscillation definition. A signal within one stance period in time domain, $s(t)$, is firstly high-pass filtered at $10 \mathrm{~Hz}$. The filtered signal, $x(t)$, is converted to frequency domain using Fast Fourier Transform. The resulting signal in frequency domain, $X(f)$, is used to construct the energy spectral density as $X(f)^{2} \cdot T_{S}^{2}$, in which $T_{S}$ is the sampling period of the signal. The total energy of oscillation of signal $s(t)$ is then the integral of the energy spectral density. The level of oscillation of a signal in one experiment session is then achieved by averaging that of every stride. . . . . . . . . . . . 162 
6.6 Estimates of optimal torque tracking errors, i.e., those of the trials with minimum errors, for all combinations of passive stiffnesses and desired torque curves. (A) Torque errors for linear curves. (B) Torque errors for piece-wise linear curves with four phases presented separately. Row one presents the absolute errors. Row two presents the percentage of absolute errors relative to the peak desired torque of the corresponding experiment session. For both curve types, experimental estimates of minimum torque tracking errors show fairly strong linear correlation with the absolute values of the difference between the passive stiffness and the desired stiffness, $\left\|K_{t}-K_{d e s}\right\|$, which agrees with Hypothesis 1 and Hypothesis 2 . . . . . . . . . . . . . . . . . . . . 164

6.7 Values for the estimates of optimal proportional gain, $K_{p}$, i.e., those of the trials with minimum errors, of various passive stiffness configurations show fairly strong inverse proportional correlation with the respective passive stiffness values for all desired curves $\left(R^{2} \geq 0.565\right)$, which agrees with Hypothesis 3. . . . . . . . . . . . . . 165

6.8 Values of gain-stiffness inverse correlation coefficient, $\sigma$, which were achieved by curve fitting in Fig. 6.7, are inversely proportional to the desired stiffness values of various curves. This agrees with Hypothesis 4. The effective desired stiffness of a piece-wise linear curve is defined the mean of desired stiffness values across four phases averaged over all the six best-performed data sets, one for each passive stiffness

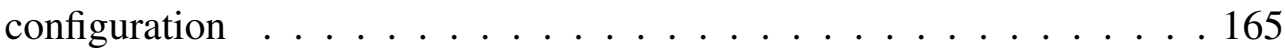


7.1 The ankle exoskeleton testbed system used in experiments of this study. A) A real-time high-speed controller DS1103 (DSpace Inc.) that reads sensory readings, generates control objectives using high level controllers and generates motor velocity commands using low level controllers. B) Motor drive. C) High speed motor. D) Bowden cable transmission. E,F) The ankle exoskeleton used. G) Base unit of Oxycon mobile that receives and processes flow rates measurements. H) Computer used to display, screen read and transmit respiratory measurements.174

7.2 The high-level controller used in experiments of this study: a desired

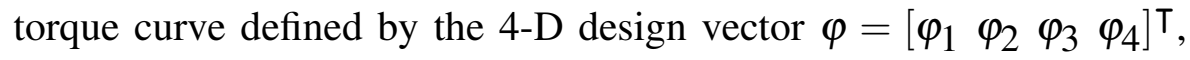
elements of which represent peak desired torque, \%stride time of peak torque happening, \%stride time of the peak torque rise time, and \%stride time of the peak torque drop time, or 'drop time' as called. In between $\left(\varphi_{2}-\varphi_{3}\right) \%$ and $\left(\varphi_{2}+\varphi_{4}\right) \%$ of stride time, the desired torque is a cubic spline that passes anchor points $\left[\varphi_{2}-\varphi_{3}, 2\right],\left[\varphi_{2}, \varphi_{1}\right]$ and $\left[\varphi_{2}+\varphi_{4}, 2\right]$. Within $\left[0 \varphi_{2}-\varphi_{3}\right] \%$ and $\left[0 \varphi_{2}+\varphi_{3} 0\right] \%$ of stride time, the desired torque is linearly interpolated between 0 and $2 \mathrm{Nm}$. . . . . . . . 177

7.3 Estimation of a single actual metabolic rate [171] from multiple raw measurements within a peroid by inversing a first order dynamics with a time constant of $42 \mathrm{~s} . \ldots \ldots$. . . . . . . . . . . . . 180

7.4 Total CMA-ES optimization evaluation time per parameter with four generations of evaluations and evaluation sample length of 2 minutes. .183 
7.5 Online optimization process using CMA-ES. Four generations, thirty two conditions, sixty-four minutes of walking were automatically generated and evaluated. The optimized high level condition is defined by the parameter means of the fifth generation which were produced at the end of the fourth one. . . . . . . . . . . . . 18

7.6 Online optimization process using Nelder-Mead method. Thirty two conditions were automatically generated and evaluated. The optimized high level condition is defined the means of the last four conditions evaluated and the new condition generated at the end of condition thirty two.

7.7 Results of online optimization validation process. A) Metabolic rates of various validation experiment sessions are presented in the sequence of test sequence. Four conditions were tested: Zero impedance mode where exoskeleton joint follows human ankle movements without exerting torques; Control condition B which was hand tuned to achieve metabolic efficiency in a prior study [172]; Optimization process identified by CMA-ES and Nelder-Mead methods. The seven experiment sessions forms two testing cycles of the four conditions to eliminate possible drifting of metabolic rates along time. For both cycles, same trends of metabolic rates were observed as zero impedance $>$ control condition B $>$ Nelder-Mead $>$ CMA-ES. B) Metabolic rates of four conditions averaged over different sessions. There is a $26.9 \%$ drop from zero impedance condition for CMA-ES and $20.5 \%$ for NelderMead condition, both outperformed control condition B which showed $16.4 \%$ drop of metabolic rate from the zero impedance condition. . . . . 187 
1 The Hill-type muscle-tendon-unit model includes a contractile element (CE), a parallel elastic element (PE) and series elastic element (SE). . . 201 


\section{Chapter 1}

\section{Introduction}

\subsection{Motivation}

Millions of people become victims of stroke or traumatic brain injuries each year. Many survivors suffer from severe neurological damage which leads to full or partial paralysis, causing losses of motion control. Clinical one-on-one rehabilitative therapy has been introduced from around 1950s to help the patients on motion recovery after stroke. However, traditional neurorehabilitation practice is inefficient due to its great labor-intensity [1]. It totally relies on therapy and evaluation process conducted by one therapist attending only one single patient at a time. The duration and frequency of these therapies are also greatly limited by the strength of practitioners. Lab-intensive activities stand for one of the key application areas for robotics.

Use of robotic devices for performance restoration or enhancement has been an area of active research for multiple decades. The history of utilizing robotics for motion assistance on humans with functional damage can be traced back to the year 1935, when a spring-driven active orthosis [2] patented in the U.S. Then in 1942, another patent 
demonstrated the first controllable human-assistive device, a hydraulically actuated orthosis [3]. Before that, the earliest human-robot cooperative work was an exoskeletonlike spring drive device aiming at lower-limb movement augmentation [4] in 1890. Lots of rehabilitation robots have been developed and tested along the way, several types of which have gradually shown their effectiveness and established their position as fundamental frameworks for devices of similar purposes. One of these devices is an upper-limb therapeutic device composed of a robot manipulator and connected to human arm in series for tracking and reaching tasks training [5]. Another example is a torque controlled powered ankle orthosis installed in parallel with human ankle for gait training $[6,7]$. However, it remains unclear in the literature what kind of controllers are feasible and effective for these devices. Many systems use ad-hoc controllers with limited understanding of the human-robot interactive system, which leads to repeated research efforts and unsatisfactory control performance.

Motivated by that, this thesis aims to identify and provide solutions to the fundamental issues in rehabilitation robot control, in particular the two groups of devices exemplified, using both theoretical and experimental methods.

\subsection{Upper-Limb Rehabilitation Robots Control Chal- lenges}

In the area of anthropomorphic robotics, upper-limb devices are more developed in terms of both theory and application. Normal robotic manipulators widely used in industry can actually be considered imitations of human arms, with or without extended degree-of-freedom. Human arm movements are mostly free motion with constrained joint angles. However, stability of the closed-loop human-robot iterative system with uncertain and changing non-linear dynamics has not been solved by the existing con- 
trollers; conflicts between human and robot movements were not handled; safety, customizability, and adjustability were not realized systematically.

\subsection{Lower-Limb Rehabilitation and Assistive Robots Con- trol Challenges}

The most important task of human legs are standing, walking and running, which involve torso-supporting, body-balancing, gait-accomplishing compromised of alternate stance and swing phases of both legs and also reactions and constraints brought over by the ground. These complexities together have contributed to the slower advance of lower-limb robotic devices. The mechanics in human gaits are not fully understood until recent years with the pioneer research of passive dynamic walking by McGeer [8]. Increasing research on details of human walking mechanism recently has boosted great interests in human lower-extremity robotic devices.

For high-energy-input low-limb exoskeletons and prostheses used for gait training and assistance, torque control is widely used. However, due to the involvement of complicated, hard-to-model, changing human-robot interactive dynamics and the timesensitive characteristic of the operation, the tracking performance of the torque controllers proposed previously was far from being optimal or satisfactory in actual human walking. Therefore, there is always motivation to improve torque tracking in these devices.

The assistance conditions from existing exoskeletons and prostheses to human bodies are mostly imitations of average human behaviors from previous studies. However, due to the complexity of human body chemical and mechanical dynamics, the suitable, beneficial or comfortable conditions vary among different people, and even at different 
times for the same person due to change of his/her physical condition or adaptation to the assistive device. It is therefore necessary to be able to rapidly identify the optimal assistance conditions from exoskeletons and prostheses for each subject at the beginning of a rather long training, assistance or experiment session.

\subsection{Main Contributions}

With theoretical, simulation and experimental work, this thesis develops low and high level control architectures for upper-limb rehabilitation robots and lower-limb assistive devices like exoskeletons and prostheses.

The main contributions of this thesis are:

- A passivity-based control architecture for upper-limb rehabilitation robots for reaching and tracking tasks made of a series connection of a robot manipulator and a human arm was developed. Through this architecture, the stability of human-robot interaction in similar systems was solved rigorously in mathematics, safety of the human body in context is automatically ensured, rehabilitation training can be automatically provided in which assistance or encouragement to human motions are given depending on how well the patient is doing, and customization can be done easily. This controller can be used on multiple devices of similar structures and purpose.

- A effective and robust control architecture for robotic legged locomotion systems was identified as model-free, integral-action-free feedback combined with iterative learning, which demonstrate tracking error as low as $1 \%$ of the peak desired torque.

- The optimal motor position iterative learning gain for torque tracking was identi- 
fied through theory and experiments as the inverse of the passive stiffness of the actuator of robotic legged locomotion systems.

- The optimal passive stiffness of series elastic actuators in robotic legged locomotion systems was identified to be equal to the desired quasi-stiffness of the operation, which is defined as the slope of the desired torque-joint angle relationship.

- An online optimization system for multivariate high level controllers of assistive robots using human metabolic measurements was developed using two algorithms: Covariance Matrix Adaptation - Evolutionary Strategies (CMA-ES) and Nelder-Mead Method. A four-parameter high level torque controller of an ankle exoskeleton was tested in experiments and showed a $26.9 \%$ of metabolic cost drop for zero impedance walking, in which the exoskeleton follows human ankle while applying zero torque.

\subsection{Structure}

Chapter 2 provides literature background for the control of upper-limb and lower-limb rehabilitation robots. It also introduces the concepts and methods used in this thesis.

Chapter 3 reviews existing control strategies of human-robot interaction in upperlimb rehabilitation robots and presents the mathematical model of the system. With this model, the chapter develops a passivity-based control structure of the system with addresses the control of motion and interaction separately and divides the work space into multiple regions with different operations based on the robot end-effector position and interaction direction. The passivity, stability and variable convergence of the humanrobot interactive system is solved and presented in this chapter. The resulting control architecture provides automatic rehabilitation training with safety, customizability, and 
generalizability to different devices. Functionality of it was tested in three-dimensional simulations and two-dimensional experiments and presented in this chapter.

Chapter 4 develops a group of torque tracking methods made of variations and combinations of model-free, model-based, feedback and feed-forward controllers were developed for robotic legged locomotion systems and presents the walking experiments on an ankle exoskeleton testbed used to compare them. The combination of modelfree, integral-action-free feedback and iterative learning was demonstrated to be the most effective and robust method from experimental results. Discussion of reasons of the effectiveness of ineffectiveness of various control methods were also provided in this chapter.

Chapter 5 further improves torque tracking in robotic legged locomotion systems on top of the robust control architecture by identifying the optimal iterative learning gain for motor position command. A theoretical analysis of the control dynamics after stabilization of the learning process is provided in this chapter, which predicts the optimal learning gain is the inverse of the passive stiffness of series elastic actuator of the device. Walking experiments involves combinations of different passive stiffness values, different desired torque profiles and different learning gains were presented and the results confirms the hypothesis.

Chapter 6 provides another attempt to further improve torque tracking in robotic legged locomotion systems by tuning the passive stiffness of the system. Theoretical conjectures and analysis are presented in the chapter, which identifies the optimal passive stiffness to be equal to the desired stiffness. The hypotheses resulted from a simple model of the system also confirms the conjecture of a constant property of a maximum change rate of torque command which results in inverse correlations between the optimal proportional gain, passive stiffness and desired stiffness of the system. Walking experiments on an ankle exoskeleton with different passive stiffness, desired quasi- 
stiffness and control gains are presented. The results confirmed the hypotheses.

Chapter 4 to 6 together presents the best control architecture, control gains and passive stiffness in robotic legged locomotion systems driven by series elastic actuators for torque tracking.

Chapter 7 develops a system for online optimization of multivariate high level controllers in human-robot interactive systems using CMA-ES and Nelder-Mead methods. Experiments with $\mathrm{N}=1$ subject on an ankle exoskeleton with a time based desired torque curve based on four parameters are presented. Results of optimization and validation processes are presented. The system is shown to be effective with a $26.9 \%$ drop of metabolic cost.

Chapter 8 summaries the work of the thesis and its conclusions, discusses potential improvements and future work. 


\section{Chapter 2}

\section{Background}

\subsection{Introduction}

To facilitate comprehensive discussions in the following chapters, this chapter provides background knowledge for the control of upper-limb and lower-limb rehabilitation robots. It also introduces the key concepts and methods used in this thesis.

\subsection{Motor Control Rehabilitation}

Motor control is crucial for human and animals in interacting with the world by realizing various actions, regulating their body balance and maintaining stability. The process of successful motor control involves the cooperation between the central nervous system and the musculoskeletal system [9]. However, tens of thousands of people lose their motor control and become paralyzed each year due to stroke or traumatic brain injury. The mechanism of their motor control loss are mostly breakage of neurological path or damage of brain motor cortex. These victims are in need of efficient and 
effective rehabilitation training to restore their lost or impaired mobility.

Modern human limb rehabilitation procedures are mostly based on a series of similar concepts referred as "neural plasticity", "brain plasticity" and "synaptic plasticity", which been proposed for over a century $[10,11]$. Synaptic plasticity was later abstracted as "neurons that fire together wire together" [12], which means that the simultaneous activation of certain neurons leads to increase in synaptic strength between them. Brain plasticity suggests that human brains have the ability to reorganize themselves. Multiple studies have demonstrated chemical and anatomic plasticity in the neural systems of animals to support these theories [13]. According to these principles, one's motor control related neurological paths and cortical maps are continuously remodeled by his/her experience, and the learning of motor skills does not happen only in one's childhood but all through his/her lifetime. This thus provides the possibility and methods for motion rehabilitation, which involves large amount of practices in order to boost the reconnection of broken neurological paths and the reorganization of brains. This process which includes repeated and concurrent happening of human intentions to move and limb movement has been introduced into therapist-assisted rehabilitation since the 1950s. However, human-based rehabilitation practice suffers from great labor intensity [1] and bad repeatability. Its effectiveness highly replies on the ability of the single therapist to conduct and evaluation the process. Its duration and frequency are also limited by the strength of the practitioners. Meanwhile, both lab-intensive and repeatitive activities stand are within the key application areas of robots. Therefore, when the field of robotics became fairly mature, robot-assisted human limb rehabilitation appeared rather naturally. In 1990s, the MIT-MANUS upper-limb therapeutic training robot was developed as a pioneer in the field [5]. 


\subsubsection{Upper-Limb Rehabilitation Robots}

Human upper-limbs have complicated and diverse movement patterns. With the cooperation of shoulder, elbow, wrist and finger joints and various muscles, upper-limbs can accomplish actions like reaching, tracking, grasping, lifting, pull, push and etc. Within these actions, reaching and tracking are most basic and fundamental. They are the first upper-limb motor skills gained by infants and usually the first ones that can be relearned after motor control loss due to relatively low requirements on muscle strength and moving accuracy. Therefore, in traditional human-based rehabilitative training, therapist-guided reaching and tracking practices were most popular at the initial stage of rehabilitation training. Imitation of this process leads to the popular configuration of stationary upper-limb rehabilitation robots which involve the serial connection of a robot manipulator and a human arm [1, 14-17].

\subsubsection{Lower-limb Rehabilitation Robots}

Human lower-limbs bear less responsibilities, which include supporting the torso, body balancing and, most importantly, locomotion. The human locomotion requires large amount of energy input, of which the majority portion is generated by ankle planterflexsors, and transmitted upwards through the ankle joints [18, 19]. Therefore, powered ankle foot orthoses, or ankle exoskeletons that provides ankle planterflexion assistance, are the most popular among lower-limb rehabilitation and assistance robots $[20,7]$.

\subsection{Interaction Control}

Rehabilitation robots are designed to assist the restoration of human motor control, and thus requires the cooperation of the robot and human body in the loop. Therefore, the 
way interaction is handled through control is crucial.

Conventional controllers treat robotics as isolated systems, and work well for motion control of unconstrained systems or those with minor bounded disturbances. However, mechanical interaction with surrounding environment is a very common feature for robotic devices. For example, robotic manipulators work properly not only by moving along the desired trajectory, but also by grasping, lifting and maybe rotating the objects, which involve interactions between the manipulators and the objects. More significantly, for devices such as assisting and rehabilitative robotics as discussed in this thesis, which require close cooperation with humans, interactions are actually a key aspect to consider in the design and control of the systems.

With control law designed while assuming the isolation of a robot from environment contacts, the addition of interaction torques can be seen as distortions to the control variables, which will affect the performance or even disrupt the stability of the closed loop system. Thus, interactions must be handled delicately.

Traditional control theories offer certain ways to deal with interactions. Firstly, it is intuitive to treat environmental interactions as disturbance and handle it by disturbance rejection method. However, the success of this method highly depends on the bounded nature of the disturbance. It fails if the interactions are too large. When robotic systems are supposed to cooperate with the environment instead of rejecting it, e.g., rehabilitative robotics, this method does not work at all. Another possible solution to the interaction problem maybe modeling uncertainty method [21], i.e., treating interactions as model uncertainty and controlling the system using robust or adaptive control methods. The first limitation of this method is that it requires that the interactions changes only the parameters but not the structure of the systems. If the structure, e.g., the degree of freedom, is varied, this method doesn't work. Another problem is that how and how much the system should react to the environmental forces can not be properly 
controlled, which makes it inefficient for robot-environmental cooperation systems.

In order to handle the reaction of robotic system to environmental forces, it is desirable to control not only the motion, but also the forces at contact points. However, force control solely was hard to realize due to the requirement of dynamic properties of both interacting systems. Thus, in the 1980s, Hogan et al. firstly brought up the concept of impedance control [22, 23].

The impedance control theory regards the robotic systems' contact points with environment as interaction ports [21]. These ports may appear as an impedance, which accept force or torque inputs and output motion, or an admittance, which accept motion inputs and output force or torques. One remarkable advantage of this method over the traditional force and motion control is that it requires the knowledge about the dynamic properties of the robot system only. Environmental properties are not needed.

\subsubsection{Rehabilitation Robot Control}

Many researchers working on rehabilitative robotic systems focus on improving the functionalities of them from the structural level by means of mechanical design. Existing controllers of the systems are mostly based on specific models generated from the devices designed in the researcher's facilities, a majority of which are directly or indirectly derived from position and impedance control theories. Marchal-Crespo and Reinkensmeyer [24] classify control strategies for performance-complementing robotic systems as assistive controllers and challenge-based controllers based on their therapeutic philosophies.

The primary control paradigm in robotic therapy development, just like physician based one-on-one therapies, is the active assist exercise. In this type of control, the robot user or the patient initiates a movement, or a predetermined desired trajectory is 
known to the robotic device. Then the device will help the user or patient to move the limbs and fulfil the task.

\subsubsection{Low-level Physical Human-Robot Interaction Control}

Traditionally, actuation impedance was made as high as possible to achieve high control bandwidth and position control strategies were most popular. However, when human body is physically interacting with robots, high actuation impedance become unsafe and uncomfortable due to the unresponsiveness of robots to human action [25, 26]. Therefore, multiple methods have been developed to reduce interface impedance and increase the reactivenss of robots in interactions with human bodies using software and hardware. Firstly, software wise, position only control strategies have been gradually replaced by hybrid control of position and force, impedance control [22] and direct torque/force control $[26,27]$. Torque control also provides a simple means of manipulating the flow of energy from the exoskeleton to the human $[28,6,29,30,172]$, which is especially useful for high power human limb movements like walking.

Hardware wise, passive compliance has been introduced in actuators to directly reduce human-robot interface impedance. Of these configurations, series elastic actuators which add series elasticity at the end effectors of the robots, are most popular [31]. The passive elasticity in the actuator decouples motor inertia from the end effector[31], physically reducing interface impedance and resulting in smaller torques when human and exoskeleton motions unexpectedly diverge. Series elastic actuation can thereby provide improved human safety [32]. and improve torque tracking performance in the face of complex, dynamic user movements [33]. 


\subsection{Key Control Algorithms}

There are some key methods used in the control of upper-limb and lower-limb rehabilitation robots in this thesis: passivity-based control, iterative learning control and Covariance Matrix Adaptation Evolution Strategy (CMA-ES), which we will briefly introduce in this section.

\subsubsection{Passivity-based Control}

One popular approach in control theory used to address the stability problem of the nonlinear systems is based on the concept of passivity. Instead of a series of states, this concept views a system as a device that transforms inputs to outputs. A passive system means one that cannot store energy more than that supplied by some "source", and the difference between stored energy and supplied energy is the dissipated energy [34-36], i.e.,

$$
V(x(t))+d(t)=\int u^{T}(\varsigma) y(\varsigma) d \varsigma
$$

in which $u \in \mathfrak{R}^{m}$ and $y \in \mathfrak{R}^{m}$ are the inputs and outputs respectively of the system. $x \in \mathfrak{R}^{m}$ is the state vector and $V(x)$ is the energy stored in the system. $d(t)$ is a nonnegative function that stands for the dissipated energy. Lyapunov theory states that the system is stable iff there is a positive definite Lyapunov function of system states such that the time deviation of it is always negative. Here in $(2.1), V(x)$ is a feasible Lyapunov candidate, and the equation can be rewritten in to

$$
\dot{V}(x(t))+\dot{d}(t)=u^{T}(t) y(t)
$$

without energy supply, i.e., $u^{T}(t) y(t)=0$, the stored energy should always be decreasing for a system to be stable in the sense of Lyapunov. In other words, $\dot{V}(x)$ should 
be negative definite for all $x$. Thus, the remaining part $\dot{d}(t)$ are expected to be always positive definite. The philosophy of passity-based control is to construct and update control low $u(t)$ in a way such that the expression $u^{T}(t) y(t)$ can be split into two parts: one is the time derivation of a Lyapunov candidate $V(x)$, and the remaining portion is negative definite.

One important property of passivity is that it is invariant under negative feedback interconnection. Therefore, the feedback interconnection of two passive systems is still a passive system, and passivity based controller can involve interconnections [35].

This method is used in this thesis to solve the stability of upper-limb rehabilitation robots used for reaching and tracking tasks.

\subsubsection{Iterative learning}

Iterative learning control is a control strategy that functions based on the repeatability of robot actions [37]. It has been widely used on industrial robots which works in highly repetitive modes. A simple control law can be expressed as

$$
u_{n+1}=u_{n}+K \cdot e_{n}
$$

in which $u_{n}$ and $u_{n+1}$ are the control inputs at the $n^{\text {th }}$ and $n+1^{\text {th }}$ periods, $K$ is a learning gain and $e_{n}$ denotes the measurement error at period $n$.

This method is use in this thesis to track the nominal torque profiles of robotic legged locomotion systems which work in cyclic mode. 


\subsubsection{Covariance Matrix Adaptation Evolution Strategy}

In chapter 7 of this thesis, we aimed to optimize multivariate high level controllers of human-robot interactive systems on line using real-time human respiratory measurements of metabolic energy consumption. This problem suffers from highly delayed and noisy measurements and expensive evaluation costs. For this reason, a gradientfree stochastic optimization method, Covariance Matrix Adaptation Evolution Strategy (CMA-ES) [38, 39], was selected to the key algorithm.

Evolution strategies are use for non-linear or non-convex continuous optimization problems. They are one group of the class of evolutionary algorithms, which are based on the principle of biological evolution: in each iteration, which is also referred as generation in this algorithm, a new population group, denoted as $x$, are generated in a stochastic way, of the current parental individuals. Then, some better performed individuals are selected to become the parents in the next generation based on their cost function value $f(x)$. Along the process, samples with better and better cost functions are generated. 


\section{Chapter 3}

\section{Upper-limb Rehabilitation Robots}

\section{Control}

\subsection{Introduction}

Neural damage in the form of stroke or traumatic brain injuries produces millions of new patients of paralysis each year, which creates great need of therapeutic training for lost or impaired motion control restoration. The theory of synaptic plasticity [10] which was later summarized as "neurons that fire together, wire together" [12] has been proposed for more than a century. According to this principle, simultaneous activation of neurons leads to increase in synaptic strength between them and therefore relearning of human movements, through "reconnection" of broken neurological path [42]

Materials in this chapter appeared in [40] : J. Zhang, C. C. Cheah, and S. H. Collins, "Stable humanrobot interaction control for upper-limb rehabilitation robotics," in Proceedings of IEEE International Conference on Robotics and Automation, Karlsruhe, Germany, May 2013, pp. 2201-2206. and [41] : J. Zhang and C. C. Cheah, "Passivity and stability of human-robot interaction control for upper-limb rehabilitation robots," IEEE Transactions on Robotics vol. 31, pp. 233- 245, 2015. 
in case of broken-neurological-path-paralysis or "reorganization" of brain [43] in case of damaged-brain-motor-cortex-paralysis. Therefore, repeated and concurrent happening of human intentions to move and limb movements is the key to recovery. Clinical one-on-one rehabilitative therapies have been introduced from the 1950s to realize this process. However, traditional human-based practice is inefficient due to its great labor intensity [1] and bad repeatability. It totally relies on therapy and evaluation process conducted by one therapist attending one single patient at a time. The duration and frequency of these therapies are also limited by the strength of practitioners. Lab-intensive activities stand for one key application area for robotics. To overcome the limitations of human-based practice, research on rehabilitative robotics has started since early 1990s, among which the MIT-MANUS upper-limb therapeutic training robot was a pioneer [5].

Robot systems can be classified into two general groups, isolated robotics that have no environmental contacts other than base support and non-isolated systems which interact with the environment besides the base support, such as rehabilitation robotics. Control theories for the former with systematic stability analysis have already been established in the literature $[44,45,35,46]$. Control of rehabilitative systems, on the contrary, is different from the traditional robotic control in the sense that it is not always a servo-mechanism [47]. Besides stability, there are two main objectives of a rehabilitative controller according to the training principle: encouraging human intentions to move and ensuring correct human movements. At early stage, patients are either fully paralyzed or generating untrustworthy signals, the training should be robot-assisting, i.e., rehabilitative robots work just like the assistive ones by treating all signals and interactions from the human as disturbances and traditional control methods are applicable. However, when motion-related neurological connection is restored but weak, the training should be robot-complementing, i.e., human intentions and movements should be encouraged and complemented rather than rejected [47]. In this case, traditional control methods are not suitable. 
The primary control paradigm in robotic therapy development is the active assist exercise [24] that emulates physician based therapies. In this type of controller, a human patient initiates a movement, or a predetermined desired trajectory is known to the robotic device. Then the device will assist the user to move the limbs and fulfill the task. Existing assistive controllers of rehabilitative systems are mostly directly or indirectly derived from motion and impedance manipulation [23]. Some are model-based controllers with force feedback [21, 48] or without force feedback [47]. Others are nonmodel-based methods with less predictable real-time interactions between patients and devices and thus less control of the training progress [49]. More early devices employ motion-only-based control and handle interactions by disturbance rejection or uncertain modeling. This method is proper at the early stage of therapy for some severely paralyzed patients when functionality loss is almost total and patients' ability to move their limbs can be ignored. Among these controllers, simple PD control [1,21] started with a dominant role. Later devices, for both upper limb [50] and lower limb [51], use similar strategies, but instead of asserting torques linearly related to position errors, more sophisticated force fields are used to build a virtual moving wall in space along the desired trajectory. This demonstrates one form of the "assist-as-needed" paradigm, which is proved to be more effective in [52].

However, in these existing designs, stability of the systems is not systematically solved with non-linearity, uncertainty, changing human dynamics or interaction forces [53, 54]. Due to the fact that rehabilitative robotics work in close contact with impaired human, safety is also a critical concern during the operation. Various causes can incur injuries to human in interaction with a robot [55] when the robot velocity, acceleration or torque exceeds certain values. Possible injuries include bone damage, joint damage and soft tissue fracture, severity of which may range from minor to fatal [55]. The situation is especially serious in case when robots work with motion-control-challenged human as in rehabilitation applications, when paralysis or spasticity causes high stiff- 
ness of human joint and inability to react fast. To maintain human safety throughout the operation, stability of the control systems must be ensured. Furthermore, the conflicting movements between human and robot should also be resolved in a stable manner to avoid large control forces and instability.

This chapter proposes an adaptive control framework for the upper-limb rehabilitative robotic systems that can easily realize training decisions made by therapists. The proposed method handles human-robot interaction in such a way that "correct" human movements are encouraged while incorrect movements are compensated to ensure the efficiency of the training process. Position-dependent stiffness and desired trajectory are proposed to resolve the possible conflicts in motions between patients and robots. The main contributions of this study include: (a) A theoretical framework which establishes system passivity and allows rigorous stability analysis of human-robot interaction in rehabilitative robotic system is presented. (b) The assist-as-needed policy is realized, while allowing customization of the controller based on different patients and their different stages to cope with the "patient cooperative training" [56]. (c) The conflicting movements between human and robot are reduced and contained in a stable manner to align with rehabilitation philosophy.

\subsection{Upper-limb Rehabilitative Robotics Dynamics}

The dynamics of an upper-limb rehabilitative robotic system consisting of a $n$-DOF robot manipulator connected to a human arm at the end can be expressed as

$$
M(q) \ddot{q}+\left[\frac{1}{2} \dot{M}(q)+S(q, \dot{q})\right] \dot{q}+g(q)=\tau+J^{\top}(q) f_{h},
$$

where $q \in \mathfrak{R}^{n}$ is the joint displacement vector and $\dot{q}=d q / d t \in \mathfrak{R}^{n}$ is the joint velocity vector; $M(q) \in \mathfrak{R}^{n \times n}$ is the manipulator inertia matrix, which is symmetric and posi- 
tive definite; $\left[\frac{1}{2} \dot{M}(q)+S(q, \dot{q})\right] \dot{q} \in \mathfrak{R}^{n}$ is the centripetal and Coriolis torques, in which $S(q, \dot{q}) \in \mathfrak{R}^{n \times n}$ is skew-symmetric; $g(q) \in \mathfrak{R}^{n}$ is the vector of gravitational torques; $\tau \in \mathfrak{R}^{n}$ stands for the control input torques applied at the manipulator joints; $f_{h} \in \mathfrak{R}^{m}$ is the force applied to robot end-effector by human and $J(q) \in \mathfrak{R}^{m \times n}$ denotes the Jacobian matrix relating joint space and task space.

\subsection{Human-Robot Interaction Involved Control}

In this section, we first identify three basic operation modes of the rehabilitative robotics based on the required capabilities. A controller consisting of a motion-related part (Section III.A) and an interaction-related part (Section III.B) is then introduced to realize the operation modes while dealing with system uncertainties and changing human forces.

Based on the required capabilities of the rehabilitative robotics, i.e., customizability, adjustability and safety, the three basic operation modes of the controller are identified as 1) a human-dominant mode in which the patient movements are trusted, encouraged thus only slightly interfered by the robot system, 2) a robot-dominant mode in which patient motion control is not reliable and desired movements are accomplished mostly by the assistance of the robot system, and 3) a safety-stop mode in which system endeffector is stopped due to the existence of conflicting human-robots movements and possible injuries to human if the robot stays active.

Human-dominant mode refers to the situation that human motion control has been partially restored. In this case, it is important that the affected human limb gains enough exercise through the therapeutic process, i.e., free limb motion should be allowed to a great extent. Meanwhile, certain level of speed regulation is beneficial to avoid abnormal movements that are too slow and too fast. Both of these two actions above can strengthen the accuracy and repeatability of human motion control in neuro-damaged 
patients' therapeutic process. Robot-dominant mode stands for a scenario that human movements are either unreliable or inefficient. Thus, assistance from the robot system is always necessary to either finish the task or put the human limb back to the correct track, both by moving the system closer to a desired trajectory. Trajectory tracking with regulation on both position and speed of the system is suitable for this stage. Besides the two normal operation modes mentioned above, sometimes, the system may drift to an extent such that the position errors are too big and resulting control torques may incur damage to human body. In this case, the robotic system should be stopped. It is also reasonable that the faster the system moves, the faster it should be stopped in this dangerous situation. Thus, damping control should be employed here.

In practice, it is feasible and important for a normal rehabilitative robot to possess the capability to work in all of the three modes, determined by the system end-effector's instant position. As shown in Fig. 3.1, when the system end-effector is close to the desired trajectory, it is in human-dominant region (H-DR); when it is in the robotdominant region (R-DR), the robot will help to push the whole system back to humandominant region; however, when the end-effector is drifted into the outer safety-stop region (S-SR), the system is stopped.

To fit in the conditions of different patients and their various recovery stages, the widths of human-dominant and robot-dominant regions are expected to be adjustable. At the early stage of the therapeutic process, human movements are minor or totally unreliable. Thus, human-dominant region should be reduced to a very small area so that robot-assistance is constantly present. The rehabilitative robot actually works as an assistive robot in that case. However, when human motion control has been recovered, human region should be made larger to allow more freedom in human movements.

This scheme is also applicable to training of patients with spasticity. Similarly, depending on the severity of the spasticity, the sizes of various regions can be fine- 


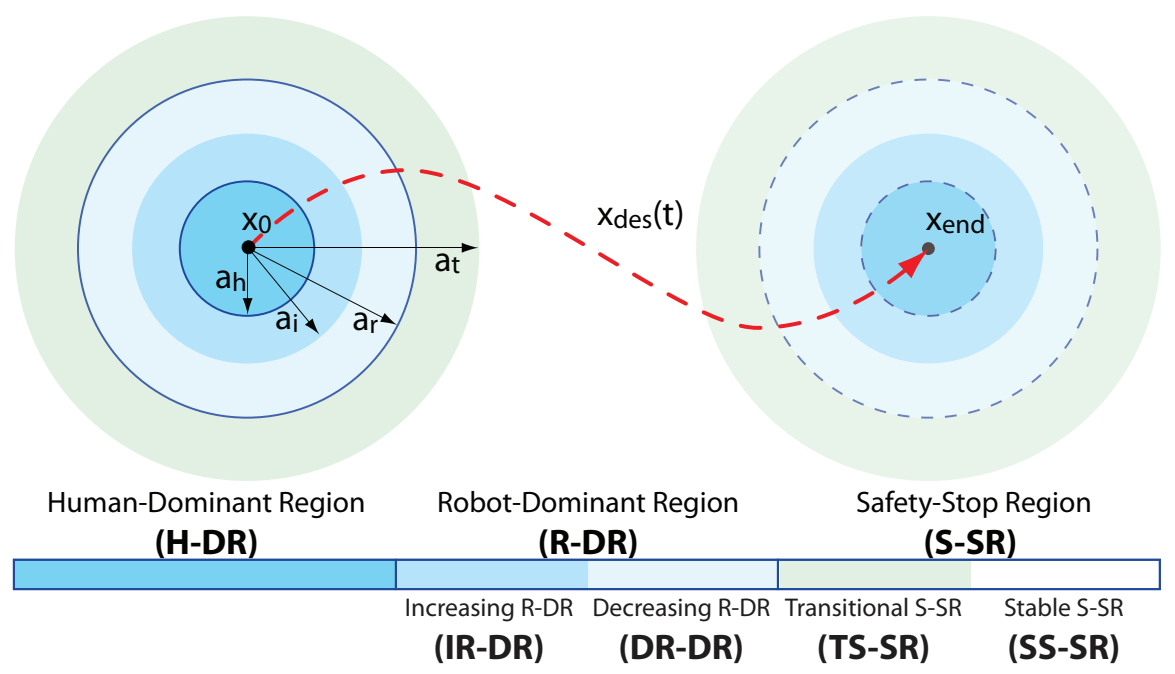

Figure 3.1: Operation regions that move along the desired trajectory $x_{\text {des }}(t)$ determined by realtime position error $\|\Delta x\|$. From inside to outside, there are three main regions, namely humandominant region (H-DR, $\|\Delta x\| \in\left[0, a_{h}\right]$ ), robot-dominant region (R-DR, $\left.\|\Delta x\| \in\left(a_{h}, a_{r}\right]\right)$ and safety-stop region (S-SR, $\|\Delta x\| \in\left(a_{r},+\infty\right)$ ). The increasing (IR-DR, $\left.\|\Delta x\| \in\left(a_{h}, a_{i}\right]\right)$ and decreasing (DR-DR, $\left.\|\Delta x\| \in\left(a_{i}, a_{r}\right]\right)$ sub-regions of R-DR are identified by increasing and decreasing stiffness (Section III.A. $\dagger 2$ ). The transitional sub-region of S-SR (TS-SR, $\|\Delta x\| \in$ $\left.\left(a_{r}, a_{t}\right]\right)$ exists to ensure smooth transition from R-DR to stable S-SR (SS-SR, $\|\Delta x\| \in\left(a_{t},+\infty\right)$. See Section III.A.†1).

tuned during initial trials. Patients with more severe spasticity should use larger robotdominant region so that reasonable amount of training can be received without constant stops in the safety-stop region. Actually, the desired region can be suspended if the patient enters safety-stop region to pause the training. The training can still be resumed by moving the arm back to the suspended region either through self-effort or with help.

To deal with the specific issues related to the rehabilitation robotics described by Eq. (3.1), the proposed controller is divided into two portions, a motion-related controller $\tau_{m}$ and an interaction-related controller $\tau_{i}$, i.e.,

$$
\tau=\tau_{m}+\tau_{i}
$$

in which $\tau_{m}$ realizes the three main operation modes and deals with modeling uncertainties, and $\tau_{i}$ deals with conflicting human-robot movements. Together, the two also 
ensure the stability of the closed-loop system.

\subsubsection{Motion-related Controller}

The motion-related controller includes a stiffness control (proportional control) term (Eq. (3.3) term $\mathfrak{a}$ ), a sliding control term (Eq. (3.3) term $\mathfrak{b}$ ) and an adaptive term (Eq. (3.3) term c). Stiffness control and sliding control terms together realize the three operation regions illustrated in Fig. 3.1 while the adaptive term deals with system uncertainties. The motion-related controller is expressed as

$$
\begin{aligned}
\tau_{m}= & \underbrace{-\mathfrak{M}^{\top}(x) J^{\top}(q) k_{p}(\delta x) \delta x}_{\text {term } \mathfrak{a}} \underbrace{-K_{S} s}_{\text {term } \mathfrak{b}} \\
& +\underbrace{Y_{d}\left(q, \dot{q}, \dot{q}_{r}, \ddot{q}_{r}\right) \hat{\theta}}_{\text {term } \mathfrak{c}},
\end{aligned}
$$

where $q \in \mathfrak{R}^{n}$ and $J(q) \in \mathfrak{R}^{n}$ are the joint displacement vector and Jacobian matrix respectively as defined in Eq. (3.1); $x=\left[x_{1}, \ldots, x_{m}\right]^{\top} \in \mathfrak{R}^{m}(m \leq 3)$ is the position of the robot end-effector in task space; $\delta x$ is a weighted position error; $\mathfrak{M}(x) \in \mathfrak{R}^{n \times n}$ is a modifier matrix; $k_{p}(\delta x) \in \mathfrak{R}$ is a non-negative position-dependent stiffness; $K_{S} \in \mathfrak{R}^{n \times n}$ is a positive sliding control gain; $s \in \mathfrak{R}^{n}$ is a sliding vector; $q_{r}$ is a virtual reference joint vector; $Y_{d}\left(q, \dot{q}, \dot{q}_{r}, \ddot{q}_{r}\right) \in \mathfrak{R}^{n \times h}$ is a regressor matrix and $\hat{\theta} \in \mathfrak{R}^{h}$ is an estimate of the unknown dynamics parameter vector $\theta \in \mathfrak{R}^{h}$.

Different from classical proportional control, term $\mathfrak{a}$ uses a position-dependent gain $k_{p}(\delta x)$. Besides, instead of the direct position error $\Delta x=x-x_{d e s}$, in which $x_{d e s}=$ $\left[x_{d e s, 1}, \ldots, x_{d e s, m}\right]^{\top}$ denotes the desired end-effector trajectory in task space, a weighted position error $\delta x$ is used, which is defined as

$$
\delta x=x-x_{d},
$$


where $x_{d}$ is a weighted trajectory expressed as

$$
x_{d}=x_{i}+w(\Delta x)\left(x_{d e s}-x_{i}\right)=x_{i}+w(\Delta x) x_{v},
$$

in which $x_{i}$ is a time-invariant reference position; $x_{v}$ is the time-varying part of $x_{d}$; $w(\Delta x)$ is a $\Delta x$-based weight vector. Note that when $w(\Delta x)=1, x_{d}=x_{d e s}$; when $w(\Delta x)=0, x_{d}=x_{i}$ and $\dot{x}_{d}=0$.

The position-dependence of $k_{p}$ and the addition of $w(\Delta x)$ to desired trajectory together realize the three main operation modes. The modifier matrix $\mathfrak{M}(x)$ is added to eliminate the use of $\ddot{x}$ in the controller, which emerges due to the usage of $w(\Delta x)$ in $x_{d}$, for noise reduction in implementation. The modifier matrix is defined as

$$
\mathfrak{M}(x)=\mathbb{I}_{n}-J^{+}(q) A(x) J(q),
$$

where $\mathbb{I}_{n}$ is a $n \times n$ identity matrix; $J^{+}(q)$ is the pseudo-inverse of $J(q) ; A(x) \in \Re^{m \times m}$ is a transition matrix defined as

$$
A(x)=\left[\begin{array}{ccc}
\frac{\partial w}{\partial x_{1}} x_{d e s, 1} & \cdots & \frac{\partial w}{\partial x_{m}} x_{d e s, 1} \\
\vdots & \ddots & \vdots \\
\frac{\partial w}{\partial x_{1}} x_{d e s, m} & \cdots & \frac{\partial w}{\partial x_{m}} x_{d e s, m}
\end{array}\right]
$$

The use of position-dependent stiffness $k_{p}$, weight factor $w(\Delta x)$ and modifier matrix $\mathfrak{M}$, also applies to the sliding vector $s$ in term $\mathfrak{b}$, which is proposed correspondingly as

$$
\begin{aligned}
s & =\dot{q}-\dot{q}_{r} \\
& =\dot{q}-\mathfrak{M}^{-1}(x)\left[J^{+}(q) \dot{x}_{f}-\alpha J^{+}(q) k_{p}(\delta x) \delta x\right]
\end{aligned}
$$

where $\alpha$ is a constant; $q_{r}$ is a virtual joint reference vector; $x_{f}$ is a virtual position reference vector defined as $\dot{x}_{f}=w(\Delta x) \dot{x}_{\text {des }}-A(x) \dot{x}_{\text {des }}$. 
The regressor $Y_{d}\left(q, \dot{q}, \dot{q}_{r}, \ddot{q}_{r}\right)$ in term $\mathfrak{c}$ is defined by

$$
M(q) \ddot{q}_{r}+\left[\frac{1}{2} \dot{M}(q)+S(q, \dot{q})\right] \dot{q}_{r}+g(q)=Y_{d}\left(q, \dot{q}, \dot{q}_{r}, \ddot{q}_{r}\right) \theta
$$

according to the linear-in-parameters property [34] of the system dynamics. By using this property, the vector of unknown system parameters, $\theta$, can be estimated by the update law:

$$
\dot{\hat{\theta}}=-L Y_{d}^{\top}\left(q, \dot{q}, \dot{q}_{r}, \ddot{q}_{r}\right) s
$$

where $L \in \mathfrak{R}^{h \times h}$ is a positive-definite and non-singular square matrix gain.

Note that this controller works with $m \leq 3$. However, for better presentation of the controller characteristics, $m=2$ is used mostly in the graphic illustrations. The threedimensional case will be later discussed and demonstrated by simulation.

At any instant time $t$ when a neuro-damage-affected human limb is in the process of finishing an action with the help of the robotic system, based on the direct position error $\Delta x$ between end-effector position $x(t)$ and desired position $x_{d e s}(t)$, the workspace is divided into three portions: human-dominant region with radius $a_{h}$, robot-dominant region with outer radius $a_{r}$ and safety-stop region where human safety will be compromised if robotic assistance is active. These three regions move along the desired trajectory together with instant $x_{d e s}(t)$ as shown in Fig. 3.1.

To meet these requirements of operation, certain conditions need to be satisfied. For proportional control term $-J^{\top}(q) k_{p}(\delta x) \delta x$ in Eq. (3.3), $k_{p}(\delta x)$ is strictly asserted to zero in human-dominant region and non-zero in robot-dominant region. With the increase of position error, $\delta x$, inside the robot region, it is also reasonable for $k_{p}(\delta x)$ to ramp up to rapidly restore the system back to the previous region. However, the value of $-J^{\top}(q) k_{p}(\delta x) \delta x$ will grow to a point where the control input torque becomes damaging to human body. Similar problem happens to term $-K_{S} s$. These concerns for 
human safety nurture the necessity of the third region, safety-stop region. In this region, $k_{p}(\delta x)$ is again set to zero to cease stiffness control, and damping will be introduced to stop the motion.

These design requirements are realized by the employment of the position-dependent trajectory weight vector $(\dagger 1)$ and the position-dependent stiffness $(\dagger 2)$.

$\dagger 1$ The position-dependent weight vector $w(\Delta x)$ is added to realize velocity tracking in human and robot-dominant regions and damping in safety-stop region by asserting $\dot{x}_{d}(t)=0$, i.e., weight vector $w(\Delta x)$ in Eq. (3.5) meets requirements

$$
w(\Delta x)= \begin{cases}1, & \mathrm{H}-\mathrm{DR} \cup \mathrm{R}-\mathrm{DR}, \\ 0, & \mathrm{~S}-\mathrm{SR} .\end{cases}
$$

in which the symbol $\cup$ denotes union operation.

To ensure smooth transition instead of asserting $w(\Delta x)=0$ in whole safety-stop region, a transitional safety-stop sub-region (TS-SR) is added at the outer edge of robot-dominant region, in which $w(\Delta x)$ smoothly changes from 1 to 0 . The subregion in which $w(\Delta x)=0$ is then named as stable safety-stop region (SS-SR). This smooth definition of $w(\Delta x)$ is

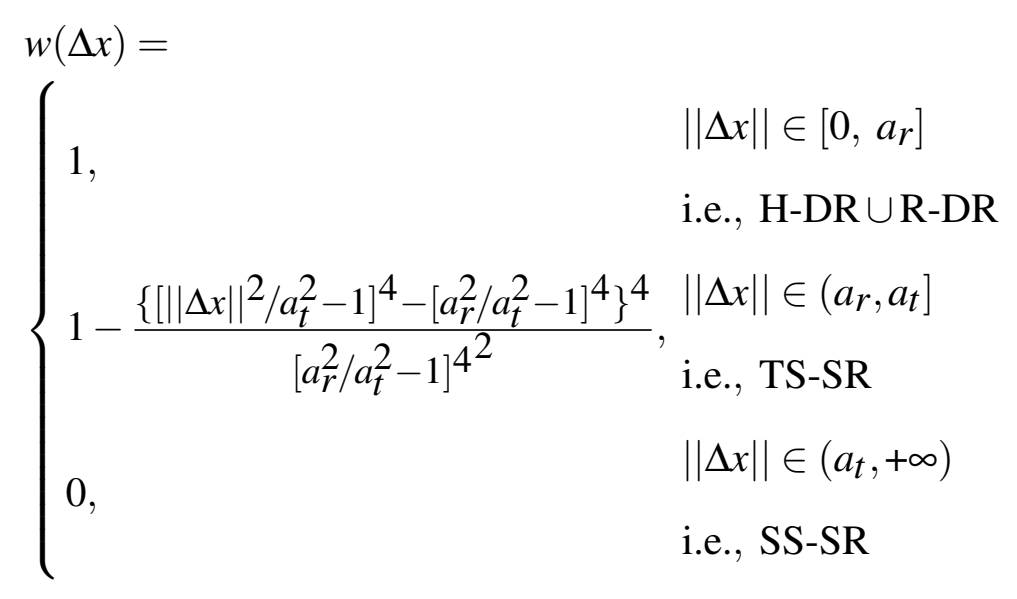

where $a_{r}$ is a positive constant standing for outer radius of robot-dominant region; 


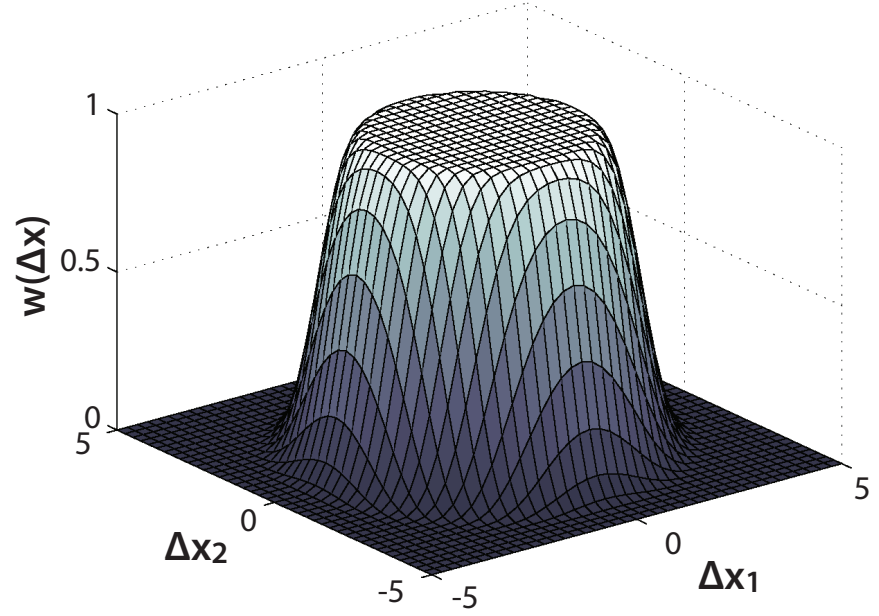

Figure 3.2: An illustration of the weight factor $w$ versus $\Delta x$ for $m=2$, with $a_{r}=2, a_{t}=4.5$. $w=1$ when $\|\Delta x\| \in\left[0, a_{r}\right]$ in the human-dominant region (H-DR) and robot-dominant region (R-DR); $w=0$ when $\|\Delta x\| \in\left(a_{t},+\infty\right)$ in the stable safety-stop region (SS-SR). $w$ smoothly transits from 1 to 0 when $\|\Delta x\| \in\left(a_{r}, a_{t}\right]$ in the transitional safety-stop region (TS-SR).

$a_{t}=a_{r}+h_{t}$ stands for outer radius of transitional safety-stop region, with a small constant of design $h_{t}$ denoting the width of the region. With this definition, an illustration of the shape of $w(\Delta x)$ for $m=2$ is available in Fig. 3.2.

While realizing velocity tracking and damping in different regions, the addition of $w(\Delta x)$ also potentially introduces $\ddot{x}$ into the controller if we define the sliding vector classically as

$$
s_{q}=\dot{q}-J^{+}(q) \dot{x}_{d}+\alpha J^{+}(q) k_{p}(\delta x) \delta x
$$

due to double differentiation of $w(\Delta x)$ in $x_{d}(t) . s_{q}$ here is labeled an intermediate sliding vector. To avoid the use of acceleration in the controller, the modifier matrix $\mathfrak{M}$ defined in Eq. (3.6) is used as detailed below.

Differentiating Eq. (3.5) with respect to time and using (3.7), we have

$$
\begin{aligned}
\dot{x}_{d} & =\dot{w}(\Delta x) x_{d e s}+w(\Delta x) \dot{x}_{d e s} \\
& =A(x) \dot{x}-A(x) \dot{x}_{d e s}+w(\Delta x) \dot{x}_{d e s} \\
& =A(x) \dot{x}+\dot{x}_{f} .
\end{aligned}
$$


From Eq. (3.6) and (3.12), the intermediate vector $s_{q}$ defined in Eq. (3.11) can be rewritten as

$$
\begin{aligned}
s_{q}= & {\left[\mathbb{I}_{n}-J^{+}(q) A(x) J(q)\right] \dot{q}-J^{+}(q) \dot{x}_{f} } \\
& +\alpha J^{+}(q) k_{p}(\delta x) \delta x \\
= & \mathfrak{M} \dot{q}-J^{+}(q) \dot{x}_{f}+\alpha J^{+}(q) k_{p}(\delta x) \delta x .
\end{aligned}
$$

Now, it is clear that the sliding vector that is defined as

$$
s=\mathfrak{M}^{-1}(x) s_{q}
$$

can be rewritten into the format of Eq. (3.8), in which the reference joint variable time derivative, $\dot{q}_{r}=\mathfrak{M}^{-1}(x)\left[J^{+}(q) \dot{x}_{f}-\alpha J^{+}(q) k_{p}(\delta x) \delta x\right]$, includes no $\dot{x}$ and therefore the term $\ddot{x}$ is eliminated in $\ddot{q}_{r}$ and hence control input $\tau$.

With this weighted desired trajectory above, considering the fact that $A(x)=$ $0_{m \times m}(m \times m$ zero matrix $)$ and thus $\mathfrak{M}(x)=\mathbb{I}_{n}$ for $w=0$ or 1 (i.e., in humandominant, robot-dominant and stable safety-stop regions), and the design requirement that $k_{p}(\delta x)=0$ in human-dominant and safety-stop regions, the sliding control portion is

$$
\begin{aligned}
& -K_{S} s=-K_{S} \mathfrak{M}^{-1}(x)\left[\dot{q}-J^{+}(q) \dot{x}_{d}+\alpha J^{+}(q) k_{p}(\delta x) \delta x\right] \\
& = \begin{cases}-K_{S}\left[\dot{q}-J^{+}(q) \dot{x}_{d e s}\right], & \|\Delta x\| \in\left[0, a_{h}\right] \\
-K_{S}\left[\dot{q}-J^{+}(q) \dot{x}_{d e s}\right. & \text { i.e., H-DR } \\
\left.+\alpha J^{+}(q) k_{p}(\delta x) \delta x\right], & \text { i.e., R-DR } \\
-K_{S} \mathfrak{M}^{-1}(x)\left[\dot{q}-J^{+}(q) \dot{x}_{d}\right], & \|\Delta x\| \in\left(a_{h}, a_{r}\right] \\
& \text { i.e., } \left.a_{t}\right] \\
-K_{S} \dot{q}, & \|\Delta x\| \in\left(a_{t},+\infty\right) \\
& \text { i.e., SS-SR }\end{cases}
\end{aligned}
$$


which implements speed control in human-dominant region and transitional safetystop region, trajectory tracking control in robot-dominant region and damping control in stable safety-stop region.

$\dagger 2$ The position-dependent stiffness $k_{p}(\delta x)$ is used instead of a constant one so that proportional control is only used in robot-dominant region, i.e.,

$$
k_{p}(\boldsymbol{\delta} x)= \begin{cases}0, & \mathrm{H}-\mathrm{DR} \\ \text { positive value, } & \mathrm{R}-\mathrm{DR} \\ 0, & \mathrm{~S}-\mathrm{SR}\end{cases}
$$

With the addition of $w(\Delta x)$, to facilitate the system passivity and stability, weighted error $\delta x$ is used for proportional control portion instead of director error $\Delta x$.

In order to obtain a continuous and differentiable stiffness that meets the requirements above, an auxiliary function is firstly defined as

$$
f_{p}(\delta x)=1-\exp \left[-a\left(\|\delta x\|^{2}-a_{h}^{2}\right)\right]
$$

and the position-dependent stiffness is described accordingly as

$$
k_{p}(\delta x)=k_{1}\left[\max \left(0, f_{p}(\delta x)\right)\right]^{2} \exp \left[-a\left(\|\delta x\|^{2}-a_{h}^{2}\right)\right]
$$

in which $a$ and $k_{1}$ are positive constants of design, and $a_{h}$ is the radius of humandominant region as mentioned earlier. An illustration of the shape of this stiffness function is available in Fig. 3.3. Note that there are two sub-regions for robotdominant region, an increasing one (IR-DR) and a decreasing one (DR-DR). The outer radius of the former, $a_{i}$, is adjustable by $a$ and $a_{h}$ according to

$$
a_{i}=\left\{\|\delta x\|: \frac{\partial k_{p}(\delta x)}{\partial \delta x}=0,\|\delta x\|>a_{h}\right\}
$$

The outer radius of the decreasing region as well as the whole robot-dominant 
(a)

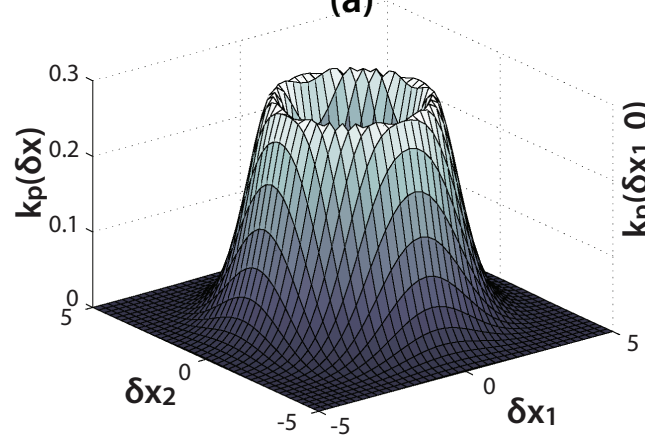

(b)

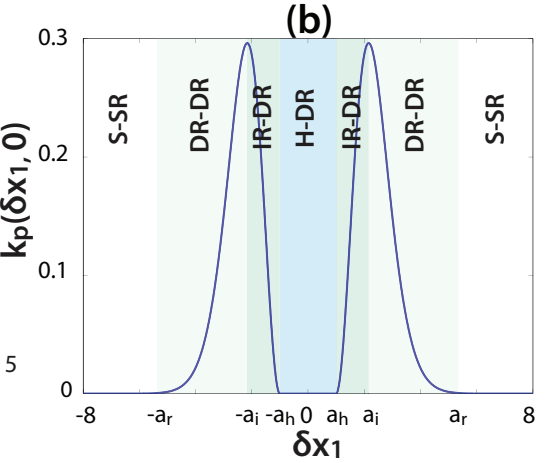

Figure 3.3: An illustration of the position-dependent stiffness $k_{p}$ versus $\delta x$ for $m=2$, with $a_{h}=$ 1, $a=0.3, k_{1}=2$. (a): A three-dimensional view; (b): A two-dimensional view: intersection of $k_{p}$ and surface $\delta x_{2}=0$. When $\|\delta x\| \in\left[0, a_{h}\right] \cup\left(a_{r},+\infty\right)$ in human-dominant region (H-DR) and safety-stop region (S-SR), $k_{p}=0$; When $\|\delta x\| \in\left(a_{h}, a_{r}\right]$ in robot-dominant region (R-DR), $k_{p}>0$. Increasing robot-dominant region (IR-DR) denotes the sub-region of R-DR in which $\partial k_{p} / \partial\|\delta x\|>0$, i.e., $k_{p}$ increases as $\|\delta x\|$ increases. Decreasing robot-dominant region (DR-DR) denotes the sub-region with $\partial k_{p} / \partial\|\delta x\|<0$.

region, $a_{r}$, is also controllable by $k_{1}, a$ and $a_{h}$. Supposing the controller processor has a minimum distinguishable signal size or quantization step size $p$, i.e., a scalar signal $y \equiv 0$ if $|y|<p$, then $a_{r}$ is determined by $a_{r}=\left\{\|\delta x\|: k_{p}(\delta x)=\right.$ $\left.p,\|\delta x\|>a_{i}\right\}$.

Note that human-dominant region provides the patients with opportunity to exercise and robot-dominant region guarantees human action correctness and reinforces human motion control. Thus, the existence of both regions is a key feature to the rehabilitative process and the relative sizes of them should reflect the recovery extent of human motion control. When human limb movement is less reliable, $a_{h}$ should be set smaller to ensure less human free motion and more robotic assistance; similarly, when human gains better motion control, $a_{h}$ should be set larger to allow more human exercise and little robot help as needed to further boost patients' recovery. When $a_{h}$ is set to zero, the system is robot-dominant, which is useful at the beginning, i.e., robot-assisting stage of the therapy. The general width of robot-dominant region can also be easily controlled by $a$. As $a$ increases, the width of robot-dominant region drops rapidly.

The fixed reference position $x_{i}$ of the weighted trajectory $x_{d}$ is chosen to be suffi- 
ciently far away from the desired path $x_{d e s}$, so that $\|\Delta x\|>a_{r}$ always guarantees $\|\delta x\|>a_{r}$ in operation, i.e., in this region, $k_{p}(\delta x)=0$.

With the $k_{p}(\delta x)$ and $x_{i}$ defined above, the stiffness control portion is

$$
\begin{aligned}
& -J^{\top}(q) k_{p}(\delta x) \delta x= \\
& \begin{cases}0, & \|\Delta x\| \in\left[0, a_{h}\right] \\
& \text { i.e., } \mathrm{H}-\mathrm{DR} \\
-J^{\top}(q) k_{p}(\delta x) \delta x, & \|\Delta x\| \in\left(a_{h}, a_{r}\right] \\
& \text { i.e., R-DR } \\
0, & \|\Delta x\| \in\left(a_{r},+\infty\right) \\
& \text { i.e., S-SR }\end{cases}
\end{aligned}
$$

in which proportional control with non-zero stiffness is deployed in robot-dominant region only.

With stiffness and desired trajectory defined above, $\tau_{m}$ is proposed as Eq. (3.3). Note that $J^{+}(q)$ and $\mathfrak{M}(x)=\mathbb{I}_{n}-J^{+}(q) A(x) J(q)$ are involved in the controller which potentially causes issues at the singularities of them. For $J^{+}(q)$, it is handled by specifying a desired trajectory $x_{d e s}(t)$ such that all singularities are in stable safety-stop region, in which case $k p(\delta x)$ and $\dot{x}_{d}$ already reduce to zero and $\mathfrak{M}=\mathbb{I}_{n}$, the motion related controller reduces to

$$
\tau_{m}=-K_{s} \dot{q}+Y_{d}(q, \dot{q}, 0,0) \hat{\theta}
$$

thus the singularity issue of the controller due to $J(q)$ is contained. Since matrix $A$ is $0_{m \times m}$ in human-dominant, robot-dominant and stable safety-stop regions, $\mathfrak{M}=\mathbb{I}_{n}$ is always invertible in these main operating regions. For the case that $A \neq 0 m \times m$ in the transitional safety-stop region, since $\mathfrak{M}=\mathbb{I}_{n}-J^{+}(q) A(x) J(q)$ is a general matrix that varies according to robot kinematics and tasks, it is difficult to derive a general analytic inverse matrix. The inverse matrix has to be analyzed according to the particu- 
lar robot structure and tasks to contain singularity issue (if any) by trajectory planning or saturating the inverse matrix. Since transitional safety-stop region is a very narrow zone between robot-dominant and stable safety-stop regions that only for the purpose of smoothing the transition, saturating $\mathfrak{M}^{-1}$ will not affect the performance in the three main regions.

\subsubsection{Interaction-related Controller}

The interaction-related controller has two objectives, to ensure the closed-loop system stability together with the motion-related controller, and to reduce the conflicts between human and robot movements. $\tau_{i}$ is proposed as a function of the human interaction force $f_{h}$, the position error $\Delta x$ and the sliding vector $s$ as

$$
\tau_{i}=J^{\top}(q) C_{x}\left(f_{h}, \Delta x, s\right)
$$

in which $C_{x}$ is the task-space interaction controller.

Research work on human science [57] has shown that human central nervous system may use a composite variable [34] consisting of tracking errors and their temporal derivatives in motion control. This composite variable can serve as either an error prediction or a criterion to be minimized. In a limb rehabilitative process assisted by a robot system, when human limb is well functioning and the robot is unactuated, the interaction force received by the robot should be fully responsible for achieving both position and speed objectives set for it, i.e., minimizing the composite variable. For the controller proposed in this article, this composite variable is represented by the task space sliding vector $s_{x}$, a combination of the transformed position and velocity errors 
as below:

$$
\begin{aligned}
s_{x} & =J(q) s=J(q) \mathfrak{M}^{-1}(x) s_{q} \\
& = \begin{cases}\delta \dot{x}, & \|\Delta x\| \in\left[0, a_{h}\right], \\
\delta \dot{x}+\alpha k_{p}(\delta x) \delta x, & \|\Delta x\| \in\left(a_{h}, a_{r}\right], \text { i.e., }, \text { R-DR } \\
J(q) \mathfrak{M}^{-1}(x) s_{q}, & \|\Delta x\| \in\left(a_{r}, a_{t}\right], \text { i.e., TS-SR } \\
\dot{x}, & \|\Delta x\| \in\left(a_{t},+\infty\right), \text { i.e., SS-SR }\end{cases}
\end{aligned}
$$

In this case, the direction of resulting force $C_{x}\left(f_{h}, \Delta x, s\right)+f_{h}$ should be aligned with the opposite of this variable.

In the safety-stop region, since the whole system is supposed to be damped to static status, $f_{h}$ is directly canceled by the controller. In human-dominant region, human movements are considered reliable to certain extent, and the controller is then introduced with three modes based on $\gamma \in[0, \pi]$, which is the magnitude of directional difference between vector $-s_{x}$ and $f_{h}$ as demonstrated in Fig. 3.4, to encourage active human movements. When $f_{h}$ orientates close enough to $-s_{x}$ with $\gamma \leq \beta$, in which $\beta$ is a threshold adjustable in $[0, \pi / 2]$, human motion is considered reliable, and the interaction force is retained. This region is called the retaining region $(R R)$. Otherwise, if $f_{h}$ direction is out of this area but still maintains an angle difference less than $\sigma$ (adjustable in $[0, \pi / 2]$ ) with one retaining region edge (denoted by unit vector either $-s_{x 1}^{\prime}$ or $-s_{x 2}^{\prime}$ ), it is in the projecting region $(P R)$ and is projected to whichever edge that is closer. Other than these two regions, $f_{h}$ is considered wrongly orientated, i.e., human movements are not reliable. Interaction is directly canceled and this area is the canceling region $(C R)$. Finally, robot-dominant region serves as a transition area. With these discussions, the interaction-related controller is developed such that

$$
\begin{aligned}
& C_{x}\left(f_{h}, \Delta x, s\right)+f_{h} \\
& =\left\{\begin{array}{lll}
\mu_{S}(s) c_{x}\left(f_{h}\right), & \|\Delta x\| \in\left[0, a_{h}\right], & \text { i.e., H-DR } \\
\mu_{S}(s) \mu_{x}(\Delta x) c_{x}\left(f_{h}\right), & \|\Delta x\| \in\left(a_{h}, a_{r}\right], & \text { i.e., R-DR } \\
0, & \|\Delta x\| \in\left(a_{r},+\infty\right), & \text { i.e., S-SR }
\end{array}\right.
\end{aligned}
$$




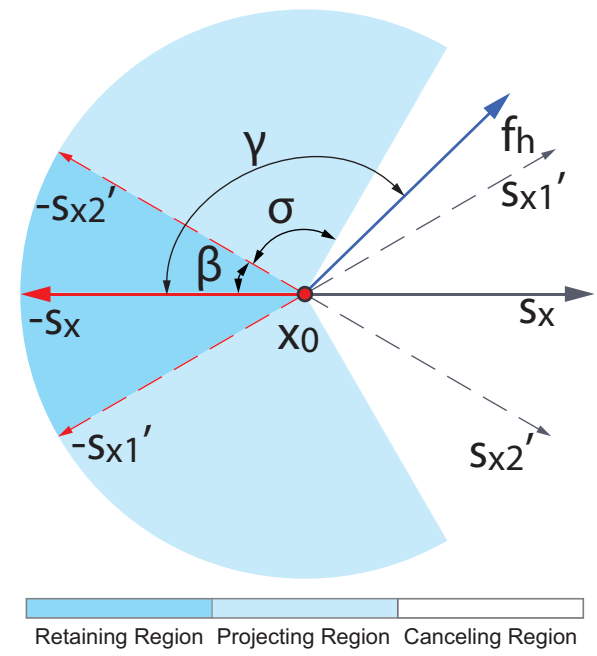

(RR)

(PR)

(CR)

Figure 3.4: Interaction force handling regions determined by the magnitude of directional difference, $\gamma$, between interaction force $f_{h}$ and the opposite of task space sliding vector $s_{x}$. When $\gamma \leq \beta, \beta \in[0, \pi / 2]$, system is in Retaining Region (RR); when $\gamma \in(\beta, \beta+\sigma], \sigma \in[0, \pi / 2]$, system is in Projecting Region (PR); otherwise, system is in Canceling Region (CR).

where

$$
c_{x}\left(f_{h}\right)= \begin{cases}f_{h}, & \gamma \in[0, \beta], \quad \text { i.e., } \mathrm{RR} \\ -s_{x}^{\prime}\left\|f_{h}\right\| \cos ^{2}\left[\frac{(\gamma-\beta) \cdot \pi}{2 \sigma}\right], & \gamma \in(\beta, \beta+\sigma], \text { i.e., } \mathrm{PR} \\ 0, & \gamma \in(\beta+\sigma, \pi], \text { i.e., } \mathrm{CR}\end{cases}
$$

with $s_{x}^{\prime}$ equals to $s_{x 1}^{\prime}$ or $s_{x 2}^{\prime}$, whichever has a closer direction with $f_{h}$. The term

$$
\mu_{x}(\Delta x)=\sin ^{2}\left[\frac{\left(a_{r}-\|\Delta x\|\right) \cdot \pi}{2\left(a_{r}-a_{h}\right)}\right],\|\Delta x\| \in\left(a_{h}, a_{r}\right] \text { only }
$$

is a position-based coefficient to ensure smooth transition between human-dominant and safety-stop regions; the saturation function

$$
\mu_{s}(s)= \begin{cases}1, & \left\|s_{x}\right\| \geq w_{s} \\ \sin ^{2}\left(\frac{\left\|s_{X}\right\| \pi}{2 w_{s}}\right), & \left\|s_{X}\right\|<w_{s}\end{cases}
$$

is added as a second coefficient of $c_{x}\left(f_{h}\right)$ to assert force-dominant controller when $s$ has a norm bigger than a small positive constant $w_{s}$, and sliding-vector-dominant controller otherwise, so that the controller is smoothed at $s=0$. Without $\mu_{S}(s)$, the resulting force 
magnitude $\left\|C_{X}+f_{h}\right\|$ is discontinuous at $s_{X}=0$, while it is smooth and continuous with $\mu_{S}(s)$. Instead of direct projection to $-s_{x}^{\prime}$ direction, a second order projection $\cos ^{2}(\bullet)$ is used in the controller to ensure its differentiability.

With definitions above, the control result $C_{x}\left(f_{h}, \Delta x, s\right)+f_{h}$ is uniformly continuous on $s, \Delta x$ and also $f_{h}$, and the term

$$
\begin{aligned}
& s^{\top} J^{\top}(q)\left[C_{x}\left(f_{h}, \Delta x, s\right)+f_{h}\right]=s_{x}^{\top}\left[C_{x}\left(f_{h}, \Delta x, s\right)+f_{h}\right]= \\
& \begin{cases}\mu_{s}(s) s_{x}^{\top} c_{x}\left(f_{h}\right), & \|\Delta x\| \in\left[0, a_{h}\right], \text { i.e., H-DR } \\
\mu_{S}(s) \mu_{x}(\Delta x) s_{x}^{\top} c_{x}\left(f_{h}\right), & \|\Delta x\| \in\left(a_{h}, a_{r}\right], \text { i.e., R-DR } \\
0, & \|\Delta x\| \in\left(a_{r},+\infty\right), \text { i.e., S-SR }\end{cases}
\end{aligned}
$$

where

$$
\begin{aligned}
& s_{x}^{\top} c_{x}\left(f_{h}\right)= \\
& \begin{cases}-\left\|s_{X}\right\|\left\|f_{S}\right\| \cos (\gamma), & \gamma \in[0, \beta] \\
-\left\|s_{X}\right\|\left\|f_{S}\right\| \cos (\beta) \cos ^{2}\left[\frac{(\gamma-\beta) \cdot \pi}{2 \sigma}\right], & \text { i.e., } \mathrm{RR} \\
& \text { i.e., } \mathrm{PR} \\
0, & \gamma \in(\beta+\beta+\sigma] \\
& \text { i.e., } \mathrm{CR}\end{cases}
\end{aligned}
$$

Since $\beta \leq \pi / 2$, Eq. (3.24) is never greater than zero.

When $m=3$, all mathematical descriptions of the controller still hold while the shapes of various regions change from two to three dimensional. For motion-related controller, human-dominant region changes from a disk to a ball; robot-dominant region changes from a ring to a hollow ball. For interaction-related part, regions change from circular sectors into cones. 


\subsection{Passivity and Stability of the System}

In this section, passivity of the closed-loop system with the proposed controller and convergence of various state variables in different operation modes are analyzed using Lyapunov method. It is shown that system passivity can be ensured and the position of the end-effector either converges to the human-dominant region, or stops in safety-stop region depending on the interactions between the human and robot.

\subsubsection{System Passivity}

By using Eq. (3.8) and substituting Eq. (3.2), (3.3) and (3.19) into Eq. (3.1), the closed-loop system dynamic equation becomes:

$$
\begin{aligned}
& M(q) \dot{s}+\left[\frac{1}{2} \dot{M}(q)+S(q, \dot{q})\right] s+\mathfrak{M}^{\top}(x) J^{\top}(q) k_{p}(\delta x) \delta x \\
& +K_{S} s+Y_{d}\left(q, \dot{q}, \dot{q}_{r}, \ddot{q}_{r}\right) \Delta \theta=J^{\top}(q)\left[C_{x}\left(f_{h}, \Delta x, s\right)+f_{h}\right],
\end{aligned}
$$

where $\Delta \theta=\theta-\hat{\theta}$. With no interaction-related controller asserted, i.e., $C_{x}\left(f_{h}, \Delta x, s\right)=$ 0 , the time integral of the inner product between an output $y=s$ and Eq. (5) yields

$$
\begin{aligned}
& \int_{0}^{t} s^{\top}(\varsigma) J^{\top}(\varsigma) f_{h}(\varsigma) d \varsigma \\
& =\int_{0}^{t}\left\{s^{\top}(\varsigma) M(q(\varsigma)) \dot{s}(\varsigma)+s^{\top}(\varsigma)\left[\frac{1}{2} \dot{M}(\varsigma)+S(\varsigma)\right] s(\varsigma)\right. \\
& \left.+\left[\dot{q}(\varsigma)-J^{+}(\varsigma) \dot{x}_{d}(\varsigma)\right)\right]^{\top}\left[\mathfrak{M}^{-1}(\varsigma)\right]^{\top} \mathfrak{M}^{\top}(\varsigma) J^{\top}(\varsigma) k_{p}(\varsigma) \delta x(\varsigma) \\
& +\left[\mathfrak{M}^{-1}(\varsigma) \alpha J^{+}(\varsigma) k_{p}(\varsigma) \delta x(\varsigma)\right]^{\top} \mathfrak{M}^{\top}(\varsigma) J^{\top}(\varsigma) k_{p}(\varsigma) \delta x(\varsigma) \\
& \left.+s^{\top}(\varsigma) K_{s} s(\varsigma)+s^{\top}(\varsigma) Y_{d}(\varsigma) \Delta \theta(\varsigma)\right\} d \varsigma .
\end{aligned}
$$


By using the skew symmetric property of $S(q, \dot{q})$, the first two terms on the right hand side of Eq. (3.27) can be expressed as

$$
\begin{aligned}
& \int_{0}^{t}\left\{s^{\top}(\varsigma) M(q(\varsigma)) \dot{s}(\varsigma)+s^{\top}(\varsigma)\left[\frac{1}{2} \dot{M}(\varsigma)+S(\varsigma)\right] s(\varsigma)\right\} d \varsigma \\
& \quad=\frac{1}{2} s^{\top}(t) M(q(t)) s(t)-\frac{1}{2} s^{\top}(0) M(q(0)) s(0) .
\end{aligned}
$$

The third term of Eq. (3.27) can be simplified as

$$
\begin{aligned}
& \int_{0}^{t}\left\{\left[\dot{q}(\varsigma)-J^{+}(\varsigma) \dot{x}_{d}(\varsigma)\right)\right]^{\top}\left[\mathfrak{M}^{-1}(\varsigma)\right]^{\top} \\
& \left.\mathfrak{M}^{\top}(\varsigma) J^{\top}(\varsigma) k_{p}(\varsigma) \delta x(\varsigma)\right\} d \varsigma \\
& \quad=\int_{0}^{t}\left\{\left[J(\varsigma)\left(\dot{q}(\varsigma)-J^{+}(\varsigma) \dot{x}_{d}(\varsigma)\right)\right]^{\top} k_{p}(\varsigma) \delta x(\varsigma)\right\} d \varsigma \\
& =\int_{0}^{t}\left\{\left[\dot{x}(\varsigma)-\dot{x}_{d}(\varsigma)\right]^{\top} k_{p}(\varsigma) \delta x(\varsigma)\right\} d \varsigma \\
& \quad=\int_{0}^{t}\left\{\delta \dot{x}^{\top}(\varsigma) k_{p}(\varsigma) \delta x(\varsigma)\right\} d \varsigma \\
& =\left.\int_{0}^{\delta x}\left\{d\left(\delta x^{\top}\right) k_{p}(\delta x) \delta x\right\}\right|_{0} ^{t} \\
& \quad=P_{t}(\delta x(t))-P_{t}(\delta x(0)),
\end{aligned}
$$

where the pseudo-potential function $P_{t}(\delta x)$ is expressed as

$$
P_{t}(\delta x)=\frac{1}{6 a} k_{1}\left[\max \left(0, f_{p}(\delta x)\right)\right]^{3}
$$

The last step of Eq. (3.29) comes from the fact that

$$
\begin{aligned}
& \frac{\partial P_{t}(\delta x)}{\partial \delta x} \\
& =\frac{1}{6 a} \cdot k_{1} \cdot 3 \cdot\left[\max \left(0, f_{p}(\delta x)\right)\right]^{2} \exp \left[-a\left(\|\delta x\|^{2}-a_{h}^{2}\right)\right] a \cdot 2 \cdot \delta x \\
& =k_{p}(\delta x) \delta x
\end{aligned}
$$

and thus

$$
\int_{0}^{\delta x}\left\{d\left(\delta x^{\top}\right) k_{p}(\delta x) \delta x\right\}=P_{t}(\delta x)
$$


Next, the fourth term can be written as

$$
\begin{aligned}
& \int_{0}^{t}\left\{\left[\mathfrak{M}^{-1}(\varsigma) \alpha J^{+}(\varsigma) k_{p}(\varsigma) \delta x(\varsigma)\right]^{\top}\right. \\
& \left.\mathfrak{M}^{\top}(\varsigma) J^{\top}(\varsigma) k_{p}(\varsigma) \delta x(\varsigma)\right\} d \varsigma \\
& \quad=\int_{0}^{t} \alpha[\delta x(\varsigma)]^{\top} k_{p}(\varsigma)^{2} \delta x(\varsigma) d \varsigma .
\end{aligned}
$$

Then, based on the system parameter estimate update law Eq. (4.20), the final term of Eq. (3.27) can be expressed as

$$
\begin{aligned}
& \int_{0}^{t}\left\{s^{\top}(\varsigma) Y_{d}(\varsigma) \Delta \theta(\varsigma)\right\} d \varsigma \\
& \quad=\int_{0}^{t}\left\{\Delta \dot{\theta}(\varsigma) L^{-1} \Delta \theta(\varsigma)\right\} d \varsigma \\
& \quad=\frac{1}{2} \Delta \theta(t)^{\top} L^{-1} \Delta \theta(t)-\frac{1}{2} \Delta \theta(0)^{\top} L^{-1} \Delta \theta(0)
\end{aligned}
$$

Combining Eq. (3.28), (3.29), (3.30) and (3.31), we have

$$
\begin{aligned}
& \int_{0}^{t} s^{\top}(\varsigma) J^{\top}(\varsigma) f_{h}(\varsigma) d \varsigma \\
& =\underbrace{V(t)-V(0)}_{\text {Stored Energy }}+\underbrace{\int_{0}^{t} W(\varsigma) d \varsigma}_{\text {Dissipated Energy }}
\end{aligned}
$$

where

$$
V=\frac{1}{2} S^{\top} M(q) s+P_{t}(\delta x)+\frac{1}{2} \Delta \theta L^{-1} \Delta \theta
$$

and

$$
W=s^{\top} K_{S} s+\alpha \delta x^{\top} k_{p}^{2}(\delta x) \delta x
$$

The pseudo-potential $P_{t}(\delta x)$, as illustrated in Fig. 3.5, has no local minimum and only global minimum inside human-dominant region, and has

$$
P_{t}(\delta x) \geq 0
$$




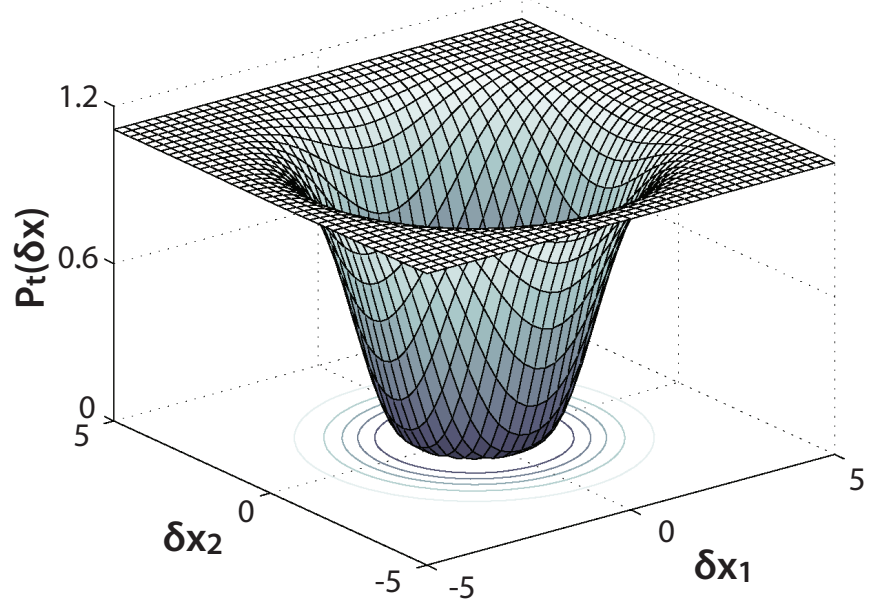

Figure 3.5: An illustration of the pseudo-energy versus the weighted position error $\delta x$ for $m=2: a_{h}=1, a=0.3, k_{1}=2$. For $\|\delta x\| \in\left[0, a_{h}\right]$, i.e., human-dominant region, $P_{t}(\delta x)=0$; For $\|\delta x\| \in\left(a_{r},+\infty\right)$, i.e., safety-stop region, $P_{t}(\delta x)$ is saturated. For $\|\delta x\| \in\left(a_{h}, a_{r}\right]$ in the robot-dominant region, $P_{t}(\delta x)$ smoothly changes from 0 to its maximum.

Then, since instant stored energy $V(t)$ in the system is composed of quadratic forms $\frac{1}{2} \Delta \theta L^{-1} \Delta \theta, s^{\top} M(q) s$ and the non-negative $P_{t}(\delta x)$, it is thus clear that

$$
V(t) \geq 0
$$

Next, the energy dissipation rate $W(s, \dot{q})$ is non-negative from Eq. (3.34). Therefore, we can prove the theorem below:

Theorem 1. The input $J^{\top}(q) f_{h}$ and output $y=s$ of the closed loop system described by Eq. (5) and (4.20) satisfy the passivity property.

Proof. Eq. (3.32) can be written as

$$
\begin{aligned}
& \int_{0}^{t} s^{\top}(\varsigma) J^{\top}(\varsigma) f_{h}(\varsigma) d \varsigma \\
& =\underbrace{V(t)-V(0)}_{\text {Stored Energy }}+\underbrace{\int_{0}^{t} W(\varsigma) d \varsigma}_{\text {Dissipated Energy }} \\
& \geq-V(0)=-k_{0}^{2},
\end{aligned}
$$

in which $k_{0}^{2}$ is a non-negative value denoting the initial system energy. Therefore, the 
input $J^{\top}(q) f_{h}$ and output $y=s$ have passive relationship.

In practical rehabilitative robotic systems, due to the existence of human-robot interaction, the term $s^{\top} J^{\top}(q) f_{h}$ will not be zero, which may result in increase of errors and storage energy. This problem is solved by the interaction-related controller $\tau_{i}$ that leads to $s^{\top} J^{\top}(q)\left[C_{x}\left(f_{h}, \Delta x, s\right)+f_{h}\right] \leq 0$ as in Eq. (3.24). Therefore, it is established that

$$
\dot{V}=-W^{\prime}=-W+s^{\top} J^{\top}(q)\left[C_{x}\left(f_{h}, \Delta x, s\right)+f_{h}\right] \leq 0,
$$

in which $W^{\prime}$ is the net energy dissipation rate.

\subsubsection{Convergence}

Different from the traditional control problem in which there are no external forces involved, the human-robot interaction may push the system end-effector into various regions. Therefore, the convergence of the state variables needs to be investigated depending on the robot end-effector position that the system starts in or enters. With the passivity conclusion, we can prove the following theory:

Theorem 2. The closed-loop system described by Eq. (5) and (4.20) guarantees the convergence of the position of end effector to the human-dominant region if it is in human or robot-dominant regions. If position of the end-effector enters the stable safetystop region, then it stops in this region.

Proof. For all cases, the function $V$ represents a Lyapunov-like function, with $V \geq 0$ and $\dot{V}=-W^{\prime} \leq 0$ from Eq. (3.35) and (3.36). Hence, it is clear that $V(t) \leq V(0)$, i.e., it is upper bounded. Since $V$ is positive-definite in $s \& \Delta \theta$, and $\dot{V}$ is negative semi-definite, we have that $s$ and $\Delta \theta$ are bounded. 


\section{$\diamond$ Human-dominant \& Robot-dominant Regions}

When robot end-effector enters or starts in these regions, it is known that

$$
\begin{aligned}
w(\Delta x)= & 1, A(x)=0_{m \times m}, \mathfrak{M}^{-1}(x)=\mathbb{I}_{n}, \\
V= & \frac{1}{2} s^{\top} M(q) s+P_{t}(\delta x)+\Delta \theta^{\top} L^{-1} \Delta \theta, \\
\dot{V}= & -s^{\top} K_{S} s-\alpha \delta x^{\top} k_{p}(\delta x)^{2} \delta x \\
& +s^{\top} J^{\top}(q)\left[C_{x}\left(f_{h}, \Delta x, s\right)+f_{h}\right], \\
s= & {\left[\dot{q}-J^{+}(q) \dot{x}_{\text {des }}+\alpha J^{+}(q) k_{p}(\delta x) \delta x\right], }
\end{aligned}
$$

and the closed-loop dynamics is

$$
\begin{aligned}
& M(q) \dot{s}+\left[\frac{1}{2} \dot{M}(q)+S(q, \dot{q})\right] s+K_{S} s+J^{\top}(q) k_{p}(\delta x) \delta x \\
& +Y_{d}\left(q, \dot{q}, \dot{q}_{r}, \ddot{q}_{r}\right) \Delta \theta-J^{\top}(q)\left[C_{x}\left(f_{h}, \Delta x, s\right)+f_{h}\right]=0 .
\end{aligned}
$$

First, the boundedness of $s$ and $\Delta \theta$ is already established. Next, $k_{p}(\delta x) \delta x$ is bounded by definition. $\dot{q}_{r}=J^{+}(q)\left[\dot{x}_{f}-\alpha k_{p}(\delta x) \delta x\right]$ is bounded if $\dot{x}_{f}=w(\Delta x) \dot{x}_{d e s}-$ $A(x) \dot{x}_{d e s}$ is bounded, which is true if $\dot{x}_{d e s}$ is bounded. Then, according to Eq. (3.8), $\dot{q}$ is also bounded. Since $\dot{q}$ is bounded and $J(q)$ is composed of trigonometric functions of $q, \dot{x}=J(q) \dot{q}$ is bounded. By using $\mathfrak{M}(x), \ddot{q}_{r}$ has been released from the dependence on $\ddot{x}$. Therefore, the boundedness of $\dot{x}, w(\Delta x)$, $\dot{w}(\Delta x), \ddot{x}_{d e s}, \mathfrak{M}(x)$ and $k_{p}(\delta x) \delta x$ suggests the boundedness of $\ddot{q}_{r}$. Thus, regressor $Y_{d}\left(q, \dot{q}, \dot{q}_{r}, \ddot{q}_{r}\right)$ is bounded. Next,

$$
\begin{aligned}
& \ddot{V}=-2 s^{\top} K_{s} \dot{s}-2 \alpha k_{p}^{2}(\delta x) \delta x^{\top} \delta \dot{x}- \\
& 2 \alpha \delta x^{\top} k_{p}(\delta x) \delta x \frac{\partial k_{p}(\delta x)}{\partial \delta x} \delta \dot{x}+\dot{s}^{\top} J^{\top}(q)\left[C_{x}\left(f_{h}, \Delta x, s\right)+f_{h}\right] \\
& +s^{\top} \dot{J}^{\top}(q)\left[C_{x}\left(f_{h}, \Delta x, s\right)+f_{h}\right]+s^{\top} J^{\top}(q)\left[\dot{C}_{x}\left(f_{h}, \Delta x, s\right)+\dot{f}_{h}\right]
\end{aligned}
$$

is bounded due to the boundedness of $\dot{s}, \delta x, \delta \dot{x}, k_{p}(\delta x), \frac{\partial k_{p}(\delta x)}{\partial \delta x}, \dot{C}_{x}\left(f_{h}, \Delta x, s\right)$ which is ensured by the definition of $C_{x}\left(f_{h}, \Delta x, s\right)$, and $\dot{f}_{h}$ due to the human motion capability limitation. According to Barbalat Lemma [34], $\dot{V} \rightarrow 0$ as 
$t \rightarrow \infty$, which implies $s \rightarrow 0$ and $k_{p}(\delta x) \delta x \rightarrow 0$ as $t \rightarrow \infty$. Thus, it is clear that $\dot{q}-J^{+}(q) \dot{x}_{d e s} \rightarrow 0$ as $t \rightarrow \infty$. We then have, $\dot{x} \rightarrow \dot{x}_{d e s}$, and $x \rightarrow$ H-DR.

Therefore, velocities of the robot inside human-dominant or robot-dominant regions converge to the desired ones $\dot{x}_{d e s}(t)$ and its end-effector goes to humandominant region with radius $a_{h}$ unless some strong external interference force it into the safety-stop region, in which case the analysis will be detailed in the next section.

\section{$\diamond$ Safety-stop Region}

If the robot end-effector enters this region accidentally or starts within it, it is known that $C_{x}\left(f_{h}, \Delta x, s\right)+f_{h}=0, k_{p}(\delta x) \delta x=0$, and $P_{t}(\delta x)=P_{t, \text { sat }}$ with $P_{t, \text { sat }}$ representing the $P_{t}(\delta x)$ value in its saturation region, which yields

$$
\begin{aligned}
& s= \\
& \begin{cases}\mathfrak{M}^{-1}(x)\left[\dot{q}-J^{+}(q) \dot{x}_{d}\right], & \|\Delta x\| \in\left(a_{r}, a_{t}\right], \text { i.e., TS-SR } \\
\dot{q}, & \|\Delta x\| \in\left(a_{t},+\infty\right), \text { i.e., SS-SR }\end{cases}
\end{aligned}
$$

and

$$
\begin{aligned}
& \dot{V}= \\
& \begin{cases}-\left[\dot{q}-J^{+}(q) \dot{x}_{d}\right]^{\top} K_{S}\left[\dot{q}-J^{+}(q) \dot{x}_{d}\right], & \|\Delta x\| \in\left(a_{r}, a_{t}\right] \\
-\dot{q}^{\top} K_{S} \dot{q}, & \|\Delta x\| \in\left(a_{t},+\infty\right) .\end{cases}
\end{aligned}
$$

Similar to the analysis of human and robot-dominant regions, with the assumption of bounded human input, it can be proved that $\dot{V} \rightarrow 0$ as $t \rightarrow \infty$ based on the definition of the controller. Therefore, in stable safety-stop region, $\dot{q} \rightarrow 0$ as $t \rightarrow \infty$, i.e., $\dot{x} \rightarrow 0$ as $t \rightarrow \infty$, due to the quadratic property of $\dot{V}$ on $\dot{q}$. It means that damping is realized in stable safety-stop region. In transitional sub-region, as $t \rightarrow \infty,\left[\dot{q}-J^{+}(q) \dot{x}_{d}\right] \rightarrow 0$, i.e., $\dot{x} \rightarrow \dot{x}_{d}$. This means velocity tracking towards $\dot{x}_{d}$ in the transitional region is realized.

Therefore, when the system is inside the stable stop region, the velocities con- 
(a)

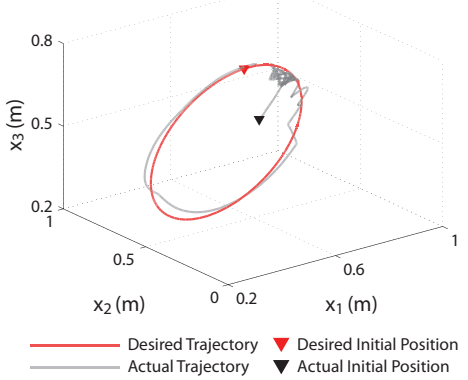

(b)

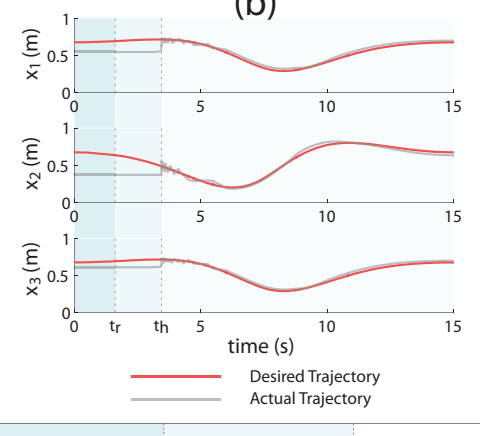

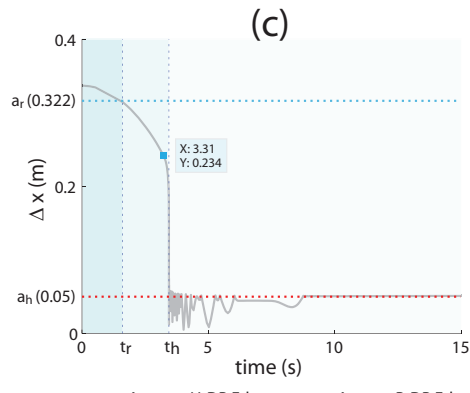

$\Delta x=$ ah, H-DR Edge $\cdots \cdots . \Delta x=$ ar, R-DR Edge

$\Delta x$ Actual Trajectory

Figure 3.6: Three-dimensional RRR-robot simulation results. (a): The desired and simulated trajectories in space; (b): The desired and simulated position trajectories of all three axes in time domain; (c): The instant distance of robot end-effector and desired central trajectory $\Delta x$ in time. From (a) and (b), it is seen that the system end-effector started at a location far away from the initial desired position $x_{\text {des }}(0)$ by design, which resulted in controller running safety-stop (S-S) mode at the beginning. As the desired trajectory $x_{d e s}$ approached the end-effector later (intentionally by trajectory planning), controller entered robot-dominant (R-D) mode at $t_{r}$. At $t_{h}$, the system first entered human-dominant region and started H-D Mode. R-D mode occasionally was still activated in this period to ensure that the system stayed in human-dominant region.

verge to zero rapidly and the system stops inside this region unless it hits the transitional region first. If by any means, system enters the narrow transitional region, the system end-effector velocity converges to the weighted desired velocity, which leads the system into either robot-dominant or stable safety-stop regions. Since $w(\Delta x)$ reduces to zero, the weighted desired velocity also reduces to zero when system approaches the stable stop region.

\subsection{Simulation and Experiments}

Simulation and experiments were performed to demonstrate the functionality and performance of the proposed controller, . Simulation was based on a 3-DOF RRR-robot to demonstrate the controller capability in three-dimensional space, i.e. $m=3$, and on curved trajectory (Section V.A). Experiments were conducted on a system consisting 
of 2-DOF planar robot connected in series with a supported human arm to demonstrate the system capability in two-dimensional space (Section V.B), i.e., $m=2$. Results and discussions are also given.

\subsubsection{Simulation}

A 3-link RRR manipulator with end-effector subjected to external force to emulate human-robot interaction was used for simulation. The three links have lengths $l_{1}=$ $l_{2}=l_{3}=0.5 \mathrm{~m}$, distances between their joints and respective center of masses $l_{c 1}=$ $l_{c 2}=l_{c 3}=0.25 \mathrm{~m}$, moments of inertia $I_{1}=I_{2}=I_{3}=\mathbb{I}_{3} \mathrm{~kg} \cdot \mathrm{m}^{2}$, and masses $m_{1}=$ $m_{2}=m_{3}=2 \mathrm{~kg} . \theta_{1}, \theta_{2}$ and $\theta_{3}$ denote the angular positions of the three joints.

A circular desired trajectory, centered at $\left[\begin{array}{lll}x_{1 c} & x_{2 c} & x_{3 c}\end{array}\right]^{\top}=\left[\begin{array}{lll}0.5 & 0.5 & 0.5\end{array}\right]^{\top} \mathrm{m}$, with orientation vectors $\left[x_{1 a} x_{2 a} x_{3 a}\right]^{\top}=[1 / \sqrt{3} 1 / \sqrt{3} 1 / \sqrt{3}]^{\top},\left[x_{1 b} x_{2 b} x_{3 b}\right]^{\top}=[1 / \sqrt{6}-2 / \sqrt{6} 1 / \sqrt{6}]^{\top}$ and radius $r=0.3 \mathrm{~m}$, as described below was used for simulation:

$$
x_{d e s, i}(t)=x_{i c}+r \cos [2 \pi \xi(t)] x_{i a}+r \sin [2 \pi \xi(t)] x_{i b},(i=1,2,3)
$$

in which the speed distribution function $\xi(t)$ was specified as

$$
\xi(t)= \begin{cases}\frac{2 t^{2}}{T^{2}}, & t \in\left[0, \frac{T}{2}\right] \\ 1-\frac{2(T-t)^{2}}{T^{2}}, & t \in\left(\frac{T}{2}, T\right]\end{cases}
$$

to ensure zero start and end velocities. $T=15 \mathrm{~s}$ is the total simulation duration. The time-invariant trajectory reference point was chosen as $x_{i}=\left[\begin{array}{lll}0 & 0 & 0\end{array}\right]^{\mathrm{T}}$.

The control parameters used were $a_{h}=0.05 \mathrm{~m}, h_{t}=0.01, k_{1}=5 \mathrm{e} 4, a=200$, $\alpha=0.5, K_{s}=50 \cdot \mathbb{I}_{3}, L=0_{4 \times 4}, \beta=0 \mathrm{rad}, \sigma=0.5 \pi \mathrm{rad}$ and $w_{s}=0.01$. A constant force $f_{h}=\left[\begin{array}{lll}10 & 10 & 10\end{array}\right]^{\top} \mathrm{N}$ was applied to demonstrate the controller capability under human interference. The outer radius of robot-dominant region was cal- 
culated as $a_{r} \approx 0.322 \mathrm{~m}$ at $p=0.1$ with the parameters provided. The initial joint position $q_{0}$ was set as $\left[\begin{array}{lll}2.3 & 2.7 & 1.2\end{array}\right]^{\top} \mathrm{rad}$, which meant an initial end-effector position $x_{0}=\left[\begin{array}{lll}0.5430 & 0.3698 & 0.6078\end{array}\right]^{\top} \mathrm{m}$ by forward kinematics. These initial position and desired trajectory were chosen such that the initial position error, $\Delta x_{0}=0.3366 \mathrm{~m}$, was larger than $a_{r}$, i.e., the system started in safety-stop region, as the path later approached $x_{0}$ and the system entered robot-dominant mode. Results of simulation, which include the desired and actual trajectories, velocities and position errors in time, are shown in Fig. 3.6.

At the beginning, being in safety-stop region, the device end-effector did not move (Fig. 3.6.b), i.e., safety-stop control mode functioned correctly. Later, the device entered robot-dominant region. Robot-dominant mode was then activated and the endeffector started to follow the desired human-dominant region. The region error with respect to the human-dominant region defined as $e_{r}(t)=\max \left(\|\Delta x(t)\|-a_{h}, 0\right)$, is available in Fig. 3.6.c.

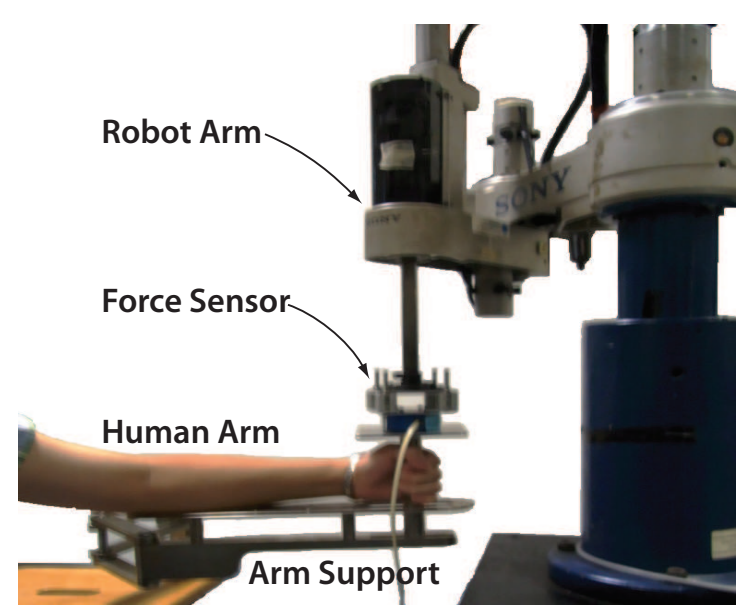

Figure 3.7: Experimental testbed: An upper-limb rehabilitative robotics composed of a 2-DOF planar robot, force sensor and a 2-DOF passive arm support.

From the simulation, the controller is able to work with three dimensional, highlycurved desired trajectory in different operation modes with force interference. Note that due to the smooth transition between different operation regions of the controller, the behavior of the closed-loop system is not hard-switched at boundaries of various 
regions. Therefore, the end-effector did not start to move towards the human-dominant region immediately after it enters the robot-dominant region (Fig. 3.6.c, $t=3.31 \mathrm{~s}$ ). Instead, it started to move when $\Delta x$ is smaller. After the system entered human-dominant region for the first time, $e_{r}$ was not always exactly zero, but sometimes slightly bigger due to force interference, in which cases the system entered robot-dominant mode and was again pulled back to human region. From $t=6.025 \mathrm{~s}$, when the system stabilized in human-dominant mode, the root mean square of region error $e_{r}$ was about $8.86 \mathrm{e}-4 \mathrm{~m}$.
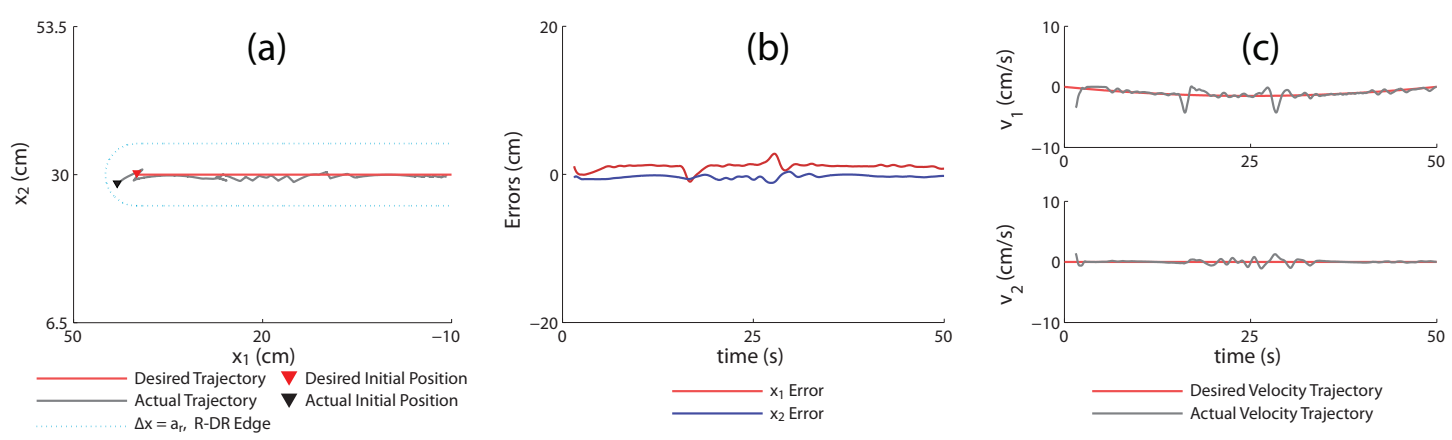

Figure 3.8: Experimental results for assistive performance test, i.e., $a_{h}=0$. (a): Desired and actual trajectories in space; (b): Position tracking errors of both $x$ and $y$ directions in time domain; (c): Desired and actual velocities of both directions in time domain, in which $v_{1}$ denotes velocity on $x_{1}$ axis and $v_{2}$ is velocity on $x_{2}$ axis.
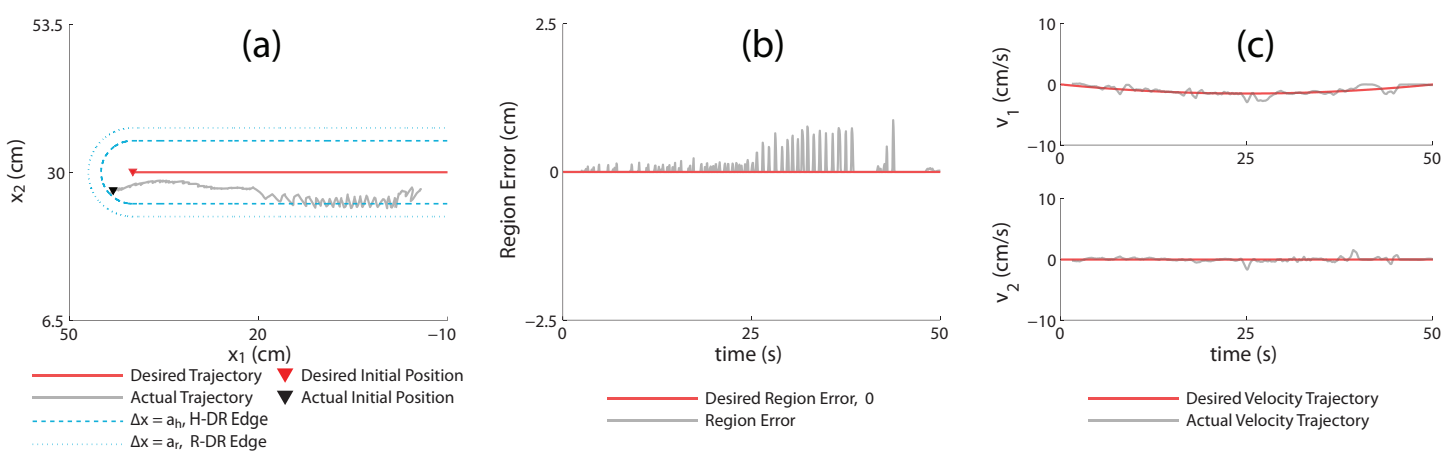

Figure 3.9: Experimental results for rehabilitative performance test with $a_{h}=0.05 \mathrm{~m}$. (a): Desired and actual trajectories in space; (b): Region errors $e_{r}=\max \left(\|\Delta x\|-a_{h}, 0\right)$ versus time; (c): Desired and actual velocities in time domain. 


\subsubsection{Experiments}

The proposed controller was implemented on an upper-limb rehabilitative robot that consisted of a 2-DOF planar robot manipulator connected to an un-actuated planar arm support in series (Fig. 3.7). A 6-axis force sensor was installed between the robot endeffector and support to measure interaction forces.

The purpose of the experiments was to test the functionality of the controller and it was not a clinical or medical trial to test the biological response of patients. Thus only one healthy subject (21 years, $1.72 \mathrm{~m}, 68 \mathrm{~kg}$, male) was involved. Experimental methods employed were approved by the university and corresponding informed consent was provided by the subject.

In all experiments with data reported, the following parameters were used: $k_{1}=$ $0.5, a=0.3, K_{S}=0.5 \cdot \mathbb{I}_{2}, \alpha=1, L=1 e-3 \cdot \mathbb{I}_{3}$, initial system parameter estimate $\hat{\theta}(0)=[0,0,0]^{\top}, p=1 e-3, \beta=\pi / 6 \mathrm{rad}, \sigma=\pi / 2 \mathrm{rad}, w_{s}=0.01$ and $h_{t}=0.01 \mathrm{~m}$. A straight desired trajectory with zero initial and final velocities and accelerations, from $\left[\begin{array}{ll}0.4 & 0.3\end{array}\right] \mathrm{m}$ to $\left[\begin{array}{ll}-0.1 & 0.3\end{array}\right] \mathrm{m}$, between time $t=0 \mathrm{~s}$ and $t=50 \mathrm{~s}$, was set as

$$
\left[\begin{array}{l}
x_{d e s, 1}(t) \\
x_{d e s, 2}(t)
\end{array}\right]=\left[\begin{array}{l}
0.4-4 e-5 \Delta t^{3}+1.2 e-6 \Delta t^{4}-9.6 e-9 \Delta t^{5} \\
0.3
\end{array}\right]
$$

with the fixed trajectory reference $x_{i}=\left[\begin{array}{ll}0 & 0\end{array}\right]^{\top}$ and $\Delta t=t-t_{0}$.

Three sets of tests were conducted. Test I investigated the assistive performance of the controller by its trajectory tracking ability starting within the robot-dominant region when human region radius was set as $a_{h}=0 \mathrm{~m}$. Outer radii of increasing and decreasing robot-dominant regions were computed as $a_{i}=0.0191 \mathrm{~m}$ and $a_{r}=0.0494 \mathrm{~m}$. Test II investigated the rehabilitative performance by human-dominant region following and human action encouragement in human region. The human-dominant region radius 
Table 3.1: RMS values for region error, $e_{r}=\max \left(\| \Delta x||-a_{h}, 0\right)$, and velocity tracking error, which is defined as $e_{v}=\left\|\dot{x}-\dot{x}_{d e s}\right\|$, in $2 \mathrm{D}$ experiments.

\begin{tabular}{l||c|c}
\hline & Test I & Test II \\
\hline RMS $e_{r}$ & $0.01305 \mathrm{~m}$ & $0.00147 \mathrm{~m}$ \\
\hline RMS $e_{v}$ & $0.0102 \mathrm{~m} / \mathrm{s}$ & $0.0035 \mathrm{~m} / \mathrm{s}$ \\
\hline
\end{tabular}

was set as $a_{h}=0.05 \mathrm{~m}$, which meant $a_{i}=0.0525 \mathrm{~m}$ and $a_{r} \approx 0.0703 \mathrm{~m}$ accordingly. The system started within human-dominant region and was intentionally pushed by the subject towards $-x_{2}$ direction (sideways) in this test. Trajectories, positional errors and velocities of Test I and II are shown in Fig. 3.8 and Fig. 3.9 respectively. The root-mean-squared region and velocity errors are given in Table 4.5. In both cases, the actual trajectories tracked the desired trajectory (I) or human-dominant region (II) closely. Region and velocity tracking errors were both smaller in Test II compared to I. Test III demonstrated the safety-stop capability of the controller using $a_{h}=0.05 \mathrm{~m}$. The end-effector started in human-dominant region and was intentionally pushed into the safety-stop region by a large human force. The system stopped in the safety-stop region after forced into this area (Fig 3.10).
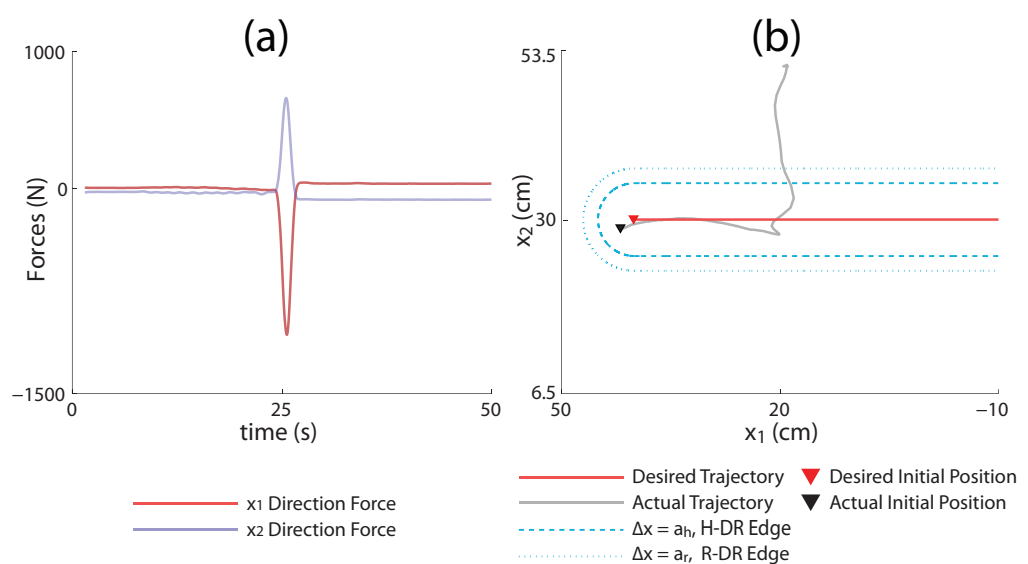

Figure 3.10: Experimental results for safety-stop performance test with $a_{h}=0.05 \mathrm{~m}$ and $a_{r}=$ $0.0703 \mathrm{~m}$. (a): The force spike applied at the handle by the subject in time domain; (b): The desired and actual spatial trajectories.

High oscillations and errors in the data were due to slow response and high frictions of the testbed, which had a control delay of $0.05 \mathrm{~s}$. Part of the velocity errors also came from the differentiation of position data. Test I \& II demonstrated the system capa- 
bilities of trajectory-tracking and region-recovery in assistive and rehabilitative modes and showed that the system possessed the ability to interact with the human in a stable manner. Test III confirmed the safety-stop capability of the controller. In Test I, velocity errors were relatively larger since the system operated in robot-dominant region where velocity tracking was less dominant. In contrast, due to the existence of humandominant region and large amount of time in it, where velocity control was dominant, Test II had smaller velocity errors. System performance is expected to improve using a faster hardware with less sensory and actuation delays.

In actual rehabilitation, one therapy does not fit the conditions of different patients at different stages. The system should thus provide flexibility for therapists to adjust and fine tune. The proposed controller, while possessing all the properties described, allows parameter adjustment. When implementing this controller on a specific device, the parameter tuning process should start with the therapist's assessment of the patient's conditions. If the patient is at early stage of the therapy and has minimal motion control, the radius of human-dominant region, $a_{h}$, should be set very small to ensure maximum robot assistance. Then, along the training process, with the improvement of patient's motion control ability, $a_{h}$ should be progressively set larger to encourage patient's active movements. With $a_{h}$ fixed, the size of robot-dominant region, denoted by $a_{r}$, should be determined to avoid injuries from large input torque or excessive human joint movements. Similarly, in interaction-related controller, $\beta$ and $\sigma$ should be made smaller when the patient has less motion control and then adjusted to a larger value when the patient improves. Based on the region sizes determined by the therapist above, location of the end-effector and direction of the human interaction then define the "correct" and "incorrect" movements. For example, an end-effector position in human-dominant region is considered correct and deviation from it is considered incorrect; an interaction force directed to $-s_{x}$ is correct while a force opposite to $-s_{x}$ is incorrect. These clinically chosen parameters are then fed into the controller system to be realized by our control framework. 
Other parameters, $k_{1}, K_{S}, L, \alpha, h_{t}, w_{s}$, are control performance related that should be tuned by control engineers before the start-up of the device and then fixed or limited to a small fine-tuning zone for the clinical training. Choice of $k_{1}$ should be balanced between maximum human bearable torque and the region tracking performance. Then $K_{S}$ and $\alpha$ are fine tuned together to achieve desired region-tracking, velocity-tracking and steady-state error performance of the controller. Short region restoration rise time can be achieved by tuning $k_{1}$ up and better velocity tracking can be done by tuning $K_{S}$ up. Smaller steady-state error can be achieved by higher $\alpha$. Finally, the parameter adaptation gain $L$ should start with very small values and be tuned up when faster adaptation is desired.

The capabilities of adjustability, customizability, safety and stability of the controller provide a dependable control platform for clinical upper-limb rehabilitative training using robotic systems. This controller is motion-dominant with the compensation of interaction-handling. Motion-dominant robot-assisted rehabilitation training is an intuitive imitation of traditional therapies, which is still used in a lot of upper-limb rehabilitative devices $[5,1,58]$. It has been shown to be an effective substitute for traditional therapist-based training [59] with comparable training results and reduced human labor-intensity. Other alternative methods start to show promises in rehabilitation, for example the manipulation of joint energy input [29], which is especially popular for lower-limb applications [6] in stance phase of walking due to the high-power-density nature of human locomotion.

\subsection{Conclusions}

In this chapter, a controller infrastructure for upper-limb rehabilitative robotics used in reaching and tracking training, which realizes three control modes, namely humandominant mode, robot-dominant mode and safety-stop mode, has been developed. With 
the employment of position-dependent stiffness and position-dependent desired trajectory, the proposed controller possesses the capability of automatic smooth transition between different operation modes to realize "assist-as-needed" strategy. By this controller, the passivity of upper-limb rehabilitative robotic system has been established; the stability of the closed-loop system has also been solved with system non-linearities, uncertainties and varying human-robot interactions; the conflicts between the movements of robot and human user have also been reduced by the proposed controller in a stable manner. With this controller, the human-robot interactive rehabilitative system moves mostly inside human-dominant region to ensure human exercise of proper amount and will be pulled back if entering robot-dominant region. Built-in safety-stop functionality is realized and triggered in case of over-sized position errors to avoid damage to human limbs. While guaranteeing all the properties described, the proposed controller allows parameter adjustment, which provides the therapists with the freedom to design patient-specific training processes. Computer simulation and pilot tests on hardware have illustrated the performances of the above-mentioned capabilities of the proposed controller.

Although this study focuses on rehabilitation robotics, the proposed controller with the safety-stop scheme can be applied to robotic devices that involve physical humanrobot interaction to ensure safety. 


\section{Chapter 4}

\section{Lower-limb Exoskeletons Torque}

\section{Control}

\subsection{Introduction}

The development of exoskeletons for performance enhancement $[62,63]$ or restoration $[64,65]$ has been an area of active research for more than fifty years. Most early exoskeletons used kinematic trajectory control, an approach that persists today [6669]. However, position control strategies tend to result in less safe and less comfortable human-robot interactions, since they can cause large forces to develop when human and robot motions differ [25, 26]. Position-controlled exoskeletons can also be less effective in rehabilitation compared to traditional human-based therapies [70].

Materials in this chapter appeared in [60]: J. Zhang, C. C. Cheah, and S. H. Collins, "Experimental comparison of torque control methods on an ankle exoskeleton during human walking," in Proceedings of the IEEE International Conference on Robotics and Automation, Seattle, WA, 2015. and [61]: J. Zhang, C. C. Cheah, and S. H. Colins, "Torque control in legged locomotion," in Book Bio-inspired Legged Locomotion, M. A. Sharbafi and A. Seyfarth, Eds. Elsevier, Aug. 2016. 
Increasingly, exoskeleton control has shifted from kinematic methods to strategies that respond more fluidly to actions of the user. One reason for this shift is the concern for human safety and comfort. Another driver is our improved understanding of the natural dynamics of human motion [71-75], which suggests a more dynamic approach to human-robot interactions than afforded by position control.

One method for improved interaction between humans and exoskeletons is impedance manipulation [22], in which the reaction of a robot to external forces is regulated rather than the resulting position trajectory [47]. Whereas position control strategies typically impose high impedance to improve trajectory tracking performance, this method allows lower impedance at the robot interface and a greater influence of human actions on the resulting motions.

Direct control of interaction forces or torques can also be used to reduce humanrobot interface impedance [26, 27]. Torque control provides a simple means of manipulating the flow of energy from the exoskeleton to the human, which can be useful in biomechanics studies $[28,6,29,30,172]$. Torque control can also be used to exploit passive dynamics or render virtual systems with alternate dynamics in humanoid robots [76], active prostheses [77-79], and exoskeletons [80-82, 7]. In exoskeletons, the quality of torque control is a limiting factor in precision of the applied intervention and can be the limiting factor in human-exoskeleton system performance.

Series elastic actuation can improve torque control in exoskeletons, which often include unknown, changing human-exoskeleton interaction dynamics. Elasticity in the actuator transmission decouples motor inertia from the exoskeleton frame [31], physically reducing interface impedance and resulting in smaller torques when human and exoskeleton motions unexpectedly diverge. Series elastic actuation can thereby provide improved human safety [32] and improve torque tracking performance in the face of complex, dynamic user movements [33]. Unlike direct-drive actuators, torque output 
in a series elastic actuator is usually not directly related to motor torque, but instead to the position of the motor relative to the joint. Motor position is therefore better correlated to load torque, especially in the presence of transmission friction. For these reasons, series elastic actuators with a motor drive running in velocity mode typically have lower actuation impedance and smoother torque tracking with lower error $[83,84]$.

Flexible Bowden cable transmissions are often used in exoskeletons to further reduce physical impedance through drive relocation. Bowden cables allow massive motor and gearbox elements to be placed in more desirable locations than the joint they actuate, resulting in reduced exoskeleton inertia. Motors can be moved proximally on the limb or body $[73,85-87]$ or off the body altogether $[88,79,7]$. Bowden cables are flexible, producing little interference with joint motions [89], but have complex stick-slip transmission dynamics that pose additional torque control challenges [85].

Unidirectional Bowden cables can completely physically isolate the human from motor inertia when desired. The capacity to become transparent, or produce zero impedance, is desirable in exoskeletons, as it is frequently useful to apply precisely zero torque to the human $[28,90-92]$. Uni-directional Bowden cables can be kept slack, preventing any torque from being transmitted regardless of human dynamics $[93,7,172]$. However, allowing the transmission to become slack introduces complex dynamics and uncertainty during re-engagement, as in other systems with intermittent contact, which can make torque control more difficult.

The human ankle produces more than half of the mechanical work of the lower limbs during walking [19] and has been a frequent target for exoskeleton assistance [20]. In fact, ankle joint assistance has led to the first systems that reduce the energy cost of walking for humans [30], including one device that does so passively [94]. Improved torque control at exoskeleton ankle joints would provide immediate benefits for such systems, and could also be beneficial at exoskeleton knee and hip joints. 
Torque control is typically found at a low level in exoskeleton control hierarchies, with higher level controllers determining behaviors and commanding desired torques. In such schemes, desired torque is not a control objective selected in advance, but rather a mid-level signal, often with complex dynamics that reflect interactions with the human user. In this manuscript, we will refer to the class of control elements that generate desired torque as high-level controllers, and to the elements that enforce desired torque, the torque controllers that are the primary focus of the study, as low-level controllers. Since the dynamics of the desired torque signal depend on the high-level control type, we expect interactions with low-level controllers that will affect torque tracking performance.

Many potential low-level control elements have been proposed for tracking torque and position in exoskeletons and series elastic actuators. Prominent categories of torque control include classical feedback, model-based control, adaptive control and iterative learning.

Classical proportional-integral-derivative (PID) feedback control, and simple variations thereon, have been widely employed in exoskeletons due to their simplicity and ease of tuning. Integral control elements are used to reduce steady state errors in series elastic actuators with consistent dynamics and low impedance [83, 84, 95, 96, 92, 97, 82]. Integration-free proportional-derivative (PD) control is often used in highimpedance exoskeletons $[50,98,99]$ and in series elastic actuators with more modeling uncertainties $[90,89]$. In cases where the derivative of the error signal is noisy, damping injection, or negative feedback on a less noisy velocity in the system, can be used instead to provide similar stabilizing effects $[44,100]$. Gain scheduling is sometimes used in the control of robots that interact with humans for improved safety or training efficiency $[52,101,40]$.

Model-based control elements are often used in robots and exoskeletons to improve 
torque-tracking performance. Approaches typically include feed-forward terms that use inverted plant dynamics to shape impedance or torque $[83,47,102,67,92,97]$. This approach works best with an accurate model of the system.

One example of adaptive control that has been applied to human-robot interaction is passivity-based control. These controllers manipulate the energy balance of the system using a system model and adaptive control elements, and can improve tracking performance with provable closed-loop stability [103]. Passivity-based control has been proposed for series elastic actuators [104] and used during human-robot interactions [41].

Variations on iterative learning derived from industrial robots have also been applied to lower-limb exoskeletons during walking $[105,92]$. This approach improves tracking performance by exploiting the cyclic nature of gait; tracking errors from past walking steps are used to predict errors in the ensuing step, and feed-forward corrections are applied. Since corrections are based on an accumulation of past errors, this approach bears some resemblance to classical integral control, with errors integrated over discrete walking steps rather than continuously in time.

High-level controllers intended to assist human walking include schemes that command desired torque based on time, joint angle, neuromuscular models, and electromyographic measurements. Perhaps the simplest way to generate desired torques is as a function of time, which can be used to regulate the relative timing of human and exoskeleton actions [30, 106, 172]. Another common method is to imitate observed relationships between human joint angles and joint torques $[107,77,108]$, which can be especially useful in regulating net joint work [89]. Virtual neuromuscular systems with complex internal dynamics have also been used to generate desired joint torques in assistive devices [109-116]. This method has demonstrated benefits in the control of adaptive prosthetic limbs [117]. Direct neuromuscular interfaces, such as through 


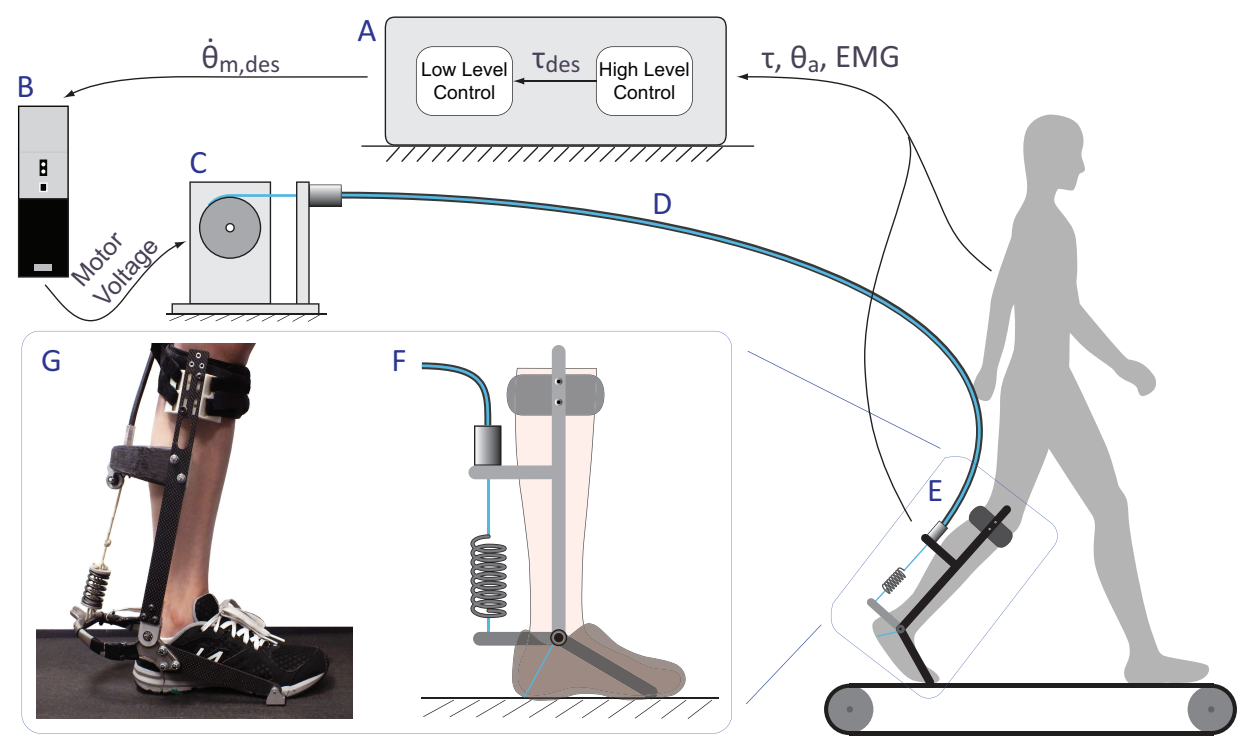

Figure 4.1: Experimental Testbed: A) High speed stand-alone real-time controller reads sensory information, computes desired torques using high-level controller, computes desired motor velocity using low level controller, and outputs desired motor velocity to the motor drive. B) Dedicated motor drive. C) Powerful off-board geared motor and pulley. D) Bowden cable transmission. E) Lightweight instrumented ankle exoskeleton. F) Enlarged schematic of the exoskeleton. G) Photograph of the exoskeleton.

electromyographic measurement of muscle activity, promise more intuitive control of exoskeletons by users $[102,20,80,118-120]$. Each of these high-level control approaches may be advantageous in some assistance paradigm, and each results in desired torque signals with different dynamics.

Many approaches to exoskeleton torque control have been established, but a more complete comparison would be helpful when designing controllers for new lower-limb exoskeleton systems. The classical feedback, model-based, adaptive and iterative learning control approaches reviewed in this section all have strengths for human-robot interaction. Several of these controllers have been tested in lower-limb exoskeletons and shown good performance [121, 88, 122, 91, 92, 82]. Comparisons across studies are made difficult, however, due to differences in protocol, performance metrics, hardware, and high-level controllers. Some results are reported for benchtop tests [123-125], which may provide more positive results than during complex interactions with humans. Some results are not reported quantitatively [121], which makes comparisons 
difficult. In some cases a small number of controllers have been tested on the same hardware [92], but in most cases torque tracking results are provided for a single controller working with a single system. This makes comparisons across studies difficult, since some portion of the differences in performance may be due to differences in the capability of the hardware used. Similarly, comparisons have been performed with different high-level controllers, which could interact with low-level controllers and contribute to differences in performance across studies. Studies comparing a wide range of torque controllers in human-interaction protocols with quantitative performance metrics, consistent hardware setups and consistent high-level controllers would help establish guidelines for selecting and tuning controllers for new lower-limb exoskeletons.

The aim of this study was to compare the tracking performance of prominent torque control methods, with multiple high-level desired torque conditions, in a single lowerlimb exoskeleton platform, during human walking. Promising methods using classical feedback, model-based, adaptive and iterative learning control elements were used. Although it was impractical to test all possible control strategies, the chosen controllers span the set of candidate methods and provide a more comprehensive test than previously available. A diverse sample of high-level controllers were used to test for interactions with low-level control dynamics and provide insights into the generality of tracking results. A single exoskeleton system was used, experimentally controlling for hardware capabilities. It was not practical to test multiple exoskeletons, however, and so results might be specific to hardware systems with similar properties. Tests were conducted while a human wore the exoskeleton and walked on a treadmill, making results relevant to conditions with complex human-robot interactions. We anticipate these results to help guide the selection and tuning of torque control elements, particularly in lower-limb exoskeletons for locomotion assistance. 


\subsection{Methods}

We compared torque tracking performance for nine common torque control methods that used combinations of classical feedback control, model-based control, adaptive control and iterative learning. These included examples of model-free and model-based feedback and feedforward control. Each low-level torque controller was tested with four high-level walking controllers that set desired torque based on time, ankle angle, a neuromuscular model, or electromyographic measurements. All controllers were implemented on a tethered ankle-foot exoskeleton with series-elastic actuation driven by a uni-directional Bowden cable, and each was tuned to minimize error. The exoskeleton was then worn by one subject who walked on a treadmill for one hundred strides at steady state under each condition, and the root mean squared errors between desired and measured torque were calculated for each stride and for an averaged stride.

\subsubsection{Exoskeleton system}

We tested controllers on a tethered ankle exoskeleton comprised of an off-board realtime control module and geared electric motor, a uni-directional Bowden cable transmission with a series spring, and an exoskeleton frame that interfaced with the human foot and shank (Fig. 4.1).

We used a dedicated real-time control system (ACE1103, dSPACE Inc.) to sample sensors at $5000 \mathrm{~Hz}$, filter sensor data at $200 \mathrm{~Hz}$, and generate desired motor velocity commands at $500 \mathrm{~Hz}$. The motor unit was composed of a low-inertia $1.6 \mathrm{~kW}$ AC servo motor and a 5:1 planetary gear, with input voltage regulated by a motor driver running in velocity control mode (BSM90N-175AD, GBSM90-MRP120-5 and MFE460A010B, Baldor Electric Co.). A digital optical encoder (E4, US Digital Corp.) measured motor 
position. As an indication of motor module performance, the $100 \%$ rise time to peak motor velocity was $0.013 \mathrm{~s}$.

A flexible uni-directional Bowden cable transmitted forces from the motor to the exoskeleton frame while minimally restricting leg motions. The cable was composed of a coiled-steel outer conduit (415310-00, Lexco Cable Mfg.) and a $0.003 \mathrm{~m}$ diameter Vectran ${ }^{\circledR}$ inner rope, and was $2 \mathrm{~m}$ in length. A series spring (DWC-148M-12, Diamond Wire Spring Co.) with an effective stiffness of $190 \mathrm{~N} \cdot \mathrm{m} \cdot \mathrm{rad}^{-1}$ (in terms of ankle rotation) was attached at the end of the rope to provide increased compliance.

The exoskeleton frame applied forces on the front of the human shank below the knee, beneath the heel, and beneath the toe, so as to generate an ankle plantarflexion torque in proportion to transmission force. Torque was measured using strain gauges (MMF003129, Micro-Measurements) applied in a full Wheatstone bridge on the heel lever, with $1000 \mathrm{~Hz}$ signal conditioning (CSG110, Futek Inc.). Joint angle was measured using a digital optical encoder (E5, US Digital Corp.). For one of the high-level controllers, we measured gastrocnemius muscle activity using a wired electromyography system (Bagnoli 4 EMG System, Delsys Inc.).

The high-level and low-level controllers, motor, transmission, exoskeleton frame and human interacted as shown in Fig. 4.2. The high-level controller used time, $t$, exoskeleton joint angle, $\theta_{e}$, or electromyography, EMG, to determine desired torque. The low-level controller regulated torque, using desired torque, $\tau_{d e s}$, measured torque, $\tau$, motor angle, $\theta_{m}$, and/or exoskeleton angle to command desired motor velocity, $\dot{\theta}_{m, d e s}$. A hardware motor driver regulated motor velocity. Motor rotations were transmitted through a Bowden cable to one end of a series spring. Together with exoskeleton rotation, this determined spring deflection, which in turn generated exoskeleton torque. Both the human and the series spring exerted torques on the exoskeleton frame, the balance of which led to exoskeleton rotation. 


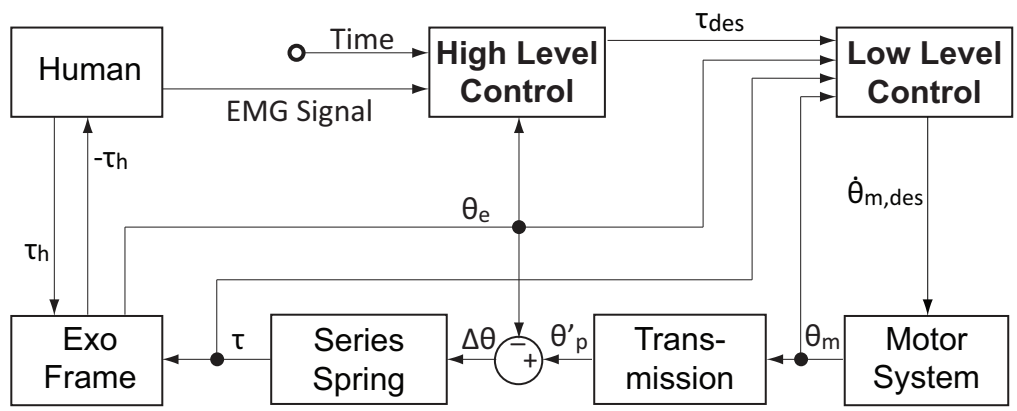

Figure 4.2: Flowchart of the control system. High Level Control and Low Level Control are the two blocks to be varied in this study.

\subsubsection{System Modeling}

We used the following simplified models of system components to aid in our understanding of the system, make reasonable choices for model-free control elements, and design model-based control elements.

$\diamond$ Motor Dynamics

Assuming armature inductance dynamics occur at a substantially higher frequency than rotor dynamics, and therefore have negligible effects, the dynamics of the motor can be written as

$$
\left\{\begin{array}{l}
K_{a} \cdot i_{a}=I_{e} \cdot N \cdot \ddot{\theta}_{p}+f_{e} \cdot N \cdot \dot{\theta}_{p}+\frac{1}{N} \cdot \tau_{o}, \\
V_{a}(t)=R_{a} \cdot i_{a}(t)+K_{b} \cdot N \cdot \dot{\theta}_{p}(t)
\end{array}\right.
$$

in which $K_{a}$ is the motor-torque constant, $i_{a}$ is the armature current, $I_{e}$ is the effective moment of inertia of the motor and gear referred to the motor shaft, $N=\dot{\theta}_{m} / \dot{\theta}_{p}$ is the gear ratio, $\theta_{m}$ is the angular position of the motor shaft, $\theta_{p}$ is the angular position of the gear output shaft, $f_{e}$ is the effective viscous friction coefficient of the combined motor and gear referred to the motor shaft, $\tau_{o}$ is output torque at the gear output pulley, $V_{a}$ is the armature voltage, $R_{a}$ is the armature resistance, and $K_{b}$ is the motor voltage constant. 
$\diamond$ Bowden Cable Model

The pulley transmits load to the Bowden cable as

$$
\tau_{o}=F \cdot r_{p}
$$

in which $r_{p}$ is the radius of the pulley attached to the gear output and $F$ is the tension in the cable on the motor side of the conduit. Making the simplifying assumption that there is no friction in the Bowden cable, the torque at the exoskeleton side of the transmission is

$$
\tau=F \cdot r_{a}
$$

in which $r_{a}$ is the lever arm at the ankle joint. We further assume that the angular excursion of the ankle joint is small, and that the lever arm is therefore approximately constant.

\section{$\diamond$ Force-Position Relationship}

Making the simplifying assumption that the Bowden cable has either spring-like compliance or negligible compliance compared to the series spring, we have

$$
F=K_{c} \cdot\left(r_{p} \cdot \theta_{p}-r_{a} \cdot \theta_{e}\right)
$$

in which $K_{C}$ is the total effective stiffness of the Bowden cable transmission and series spring and $\theta_{p}$ and $\theta_{e}$ are the pulley and exoskeleton joint angles relative to a position at which the Bowden cable begins to go slack.

$\diamond$ Torque-Angle Relationship 
Defining the gear ratio of the transmission, $R$, as

$$
R=\frac{r_{a}}{r_{p}}
$$

the torque applied by the exoskeleton can be written as

$$
\begin{aligned}
\tau & =F \cdot r_{a} \\
& =r_{p} \cdot r_{a} \cdot K_{c}\left[\theta_{p}-\frac{r_{a}}{r_{p}} \theta_{e}\right] \\
& =K_{t}\left(\theta_{p}-\theta_{e} R\right)
\end{aligned}
$$

with transmission stiffness, $K_{t}$, defined as

$$
K_{t}=r_{p} \cdot r_{a} \cdot K_{c}
$$

relating torque at the exoskeleton to the angles of the motor output pulley and exoskeleton joint.

$\diamond$ Device Joint Dynamics

Applying Newton's Second Law to the exoskeleton, we have

$$
\tau-\tau_{h}-B_{e} \cdot \dot{\theta}_{e}=I_{e} \cdot \ddot{\theta}_{e}
$$

where $\tau_{h}$ is the torque applied to the exoskeleton by human body, $B_{e}$ is the exoskeleton joint damping coefficient, and $I_{e}$ is the moment of inertia of the exoskeleton.

$\diamond$ Motor Velocity Control Dynamics

We operated the motor and commercial motor driver in velocity control mode, which tends to result in lower actuation impedance and better torque tracking $[83,84]$ in series elastic actuators. However, we did not have access to the proprietary controller used by the motor driver. The precise relationship between 
desired motor velocity, $\dot{\theta}_{m, d e s}$, and input voltage to the motor, $V_{a}$, was therefore unknown. From Eq. (4.1), we can derive the relationship between input voltage and actual motor velocity as

$$
\begin{aligned}
V_{a} & =\frac{R_{a} I_{e} N}{K_{a}} \ddot{\theta}_{p}+\left(\frac{R_{a} f_{e} N}{K_{a}}+K_{b} N\right) \dot{\theta}_{p}+\frac{R_{a}}{K_{a}} \tau_{o} \\
& =\frac{R_{a} I_{e}}{K_{a}} \ddot{\theta}_{m}+\left(\frac{R_{a} f_{e}}{K_{a}}+K_{b}\right) \dot{\theta}_{m}+\frac{R_{a}}{K_{a}} \tau_{o}
\end{aligned}
$$

When the angular acceleration is zero, this reduces to

$$
V_{a}=\left(\frac{R_{a} f_{e}}{K_{a}}+K_{b}\right) \dot{\theta}_{m}+\frac{R_{a}}{K_{a}} \tau_{o}
$$

For most motors, the contribution of armature resistance to voltage drop is small at moderate speeds. Neglecting this term, we have

$$
V_{a}=\left(\frac{R_{a} f_{e}}{K_{a}}+K_{b}\right) \dot{\theta}_{m}
$$

and input voltage and motor velocity are linearly related at moderate, steady speed.

\subsubsection{Control Issues}

While the dynamic models described by Eq. (4.1)-(4.8) capture the basic properties of the system, they do not address its full complexity. There are still uncertain or changing dynamics that are difficult to model, which contribute to most of the challenges of the control problem we are addressing.

$\diamond$ Bowden Cable Nonlinearities and Stiction

For simplicity, we modeled the Bowden cable as a frictionless linear spring, but its stiffness is actually nonlinear and there are substantial frictional effects. The 
cable is stiffening, exhibiting greater local stiffness at high loads. This can be seen in the torque versus ankle angle curves generated by fixing the motor and passively flexing the exoskeleton during walking (Fig. 6.3). The cable warms over the course of a many strides, which decreases its overall stiffness. It exhibits creep, which increases the slack length. If the cable is allowed to go slack, the state corresponding to re-engagement is uncertain. There is substantial friction in the cable, including dissipation with characteristics of Coloumb friction, viscous damping, and stiction, some of which are visible in Fig. 6.3. The cable heats over the course of many strides, which increases overall friction. Stiction leads to sudden changes in cable force, and propagation of the slipping point along the cable makes these changes unpredictable. These transmission properties are complex, nonlinear and time varying.

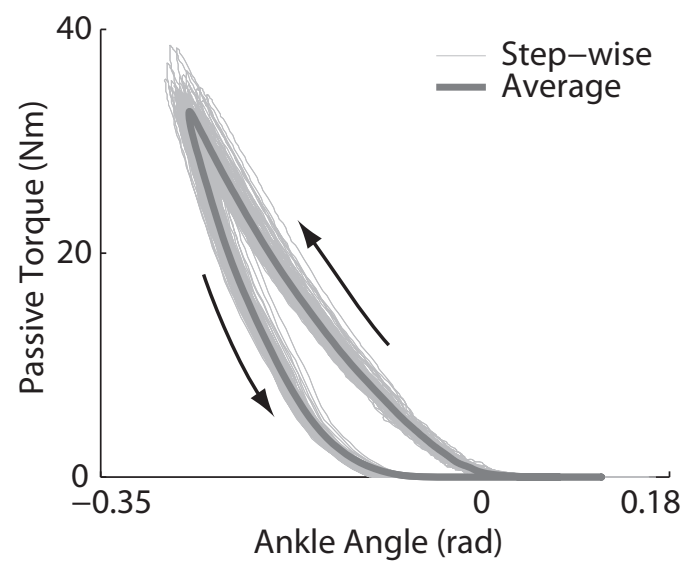

Figure 4.3: Complex torque versus exoskeleton ankle joint angle relationship when motor position was fixed and the exoskeleton was passively flexed for one hundred strides.

$\diamond$ Human-Robot Interaction and Human Adaptation

The exoskeleton frame contacts the soft tissues and muscles of the human body using flexible straps. This interface is complex and nonlinear, with low overall impedance. For example, muscle activity beneath the straps substantially affects stiffness and damping at the interface. Straps may also shift on the limb, altering 


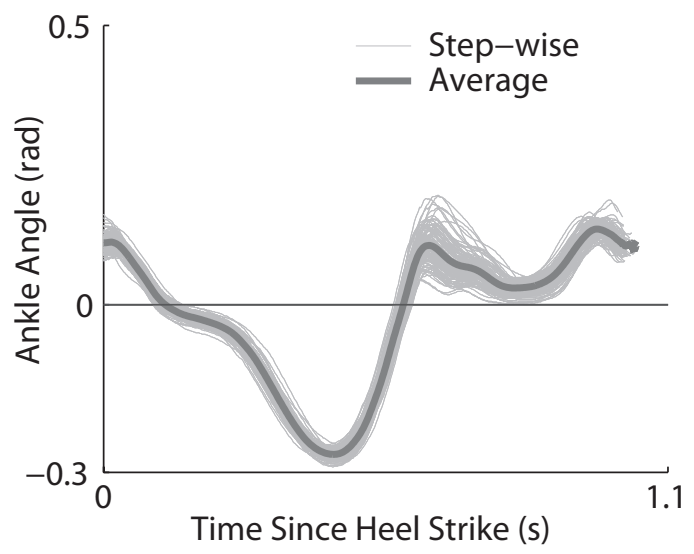

Figure 4.4: Variability in exoskeleton ankle joint angle trajectory during one hundred strides of walking with the motor fixed and the exoskeleton passively flexing.

lever arms and engaging different tissues. Human kinematics, kinetics and underlying neural and muscular activity also vary in time and across steps. This can be seen in the variations in ankle joint angle curves over many steps, even when the motor is fixed (Fig. 4.4).

$\diamond$ Communication and Motor Delays

Delays in generating desired motor position also pose a control challenge. A portion of these delays can come from communication between subsystems. For example, in the hardware used in this study there was a 6 ms closed-loop communication delay. Another effective delay comes from accelerating the motor rotor. For the hardware used in this study the motor velocity rise time was about $7 \mathrm{~ms}$. These delays cause feedback controllers to become unstable as gains are increased, limiting closed-loop performance.

An effective low-level torque controller must accommodate these complex, nonlinear, time-varying system features. 


\subsubsection{Controllers}

We tested torque tracking performance with nine prominent low-level torque control methods. Low-level controllers were selected based on prominence in the literature, expected performance based on system modeling, and the results of pilot testing. They included model-free and model-based feedback and feedforward elements. Desired torque was set with each of four high-level exoskeleton control strategies, chosen based on prominence in the literature. High-level controllers set desired torque based on time, joint angle, a neuromuscular model or electromyography.

\section{Low-Level Torque Controllers}

\section{Motor Velocity Control}

All torque controllers investigated in this study included motor velocity control performed by a dedicated hardware motor controller. Series elastic actuators with a drive running in velocity mode typically have lower actuation impedance and smoother torque tracking with lower error $[83,84]$ than when torque is commanded to the drive. With series elastic actuation, controlling motor velocity is similar to controlling the rate of change of exoskeleton torque, since torque is approximated by the product of series stiffness and the difference between motor angle and exoskeleton joint angle (Eq. 4.6). Desired motor velocity was calculated as:

$$
\begin{aligned}
\dot{\theta}_{m, d e s} & =\frac{1}{T} \cdot \Delta \theta_{m, d e s} \\
& =\frac{N}{T} \cdot \Delta \theta_{p, d e s}
\end{aligned}
$$

where $\dot{\theta}_{m, d e s}$ is commanded motor velocity, $T$ is a gain related to rise time, $\Delta \theta_{m, d e s}$ is desired change in motor position, $N$ is the motor gear ratio, and 
$\Delta \theta_{p, d e s}$ is desired change in pulley position, determined by one of the low-level torque controllers described below. The value of $T$ was tuned so as to minimize motor position rise time without causing oscillations during torque tracking.

\section{Model-Free Feedback Control}

The first group of torque controllers used model-free feedback control, comprising variations on classical proportional-integral-derivative control. Gains were tuned systematically using model-free procedures. Following tuning and pilot testing, four low-level controllers were experimentally compared, ${ }_{L}{ }^{1-} L^{4}$.

\section{$L^{1}$ : Proportional Control with Damping Injection $\left(\mathrm{PD}^{*}\right)$}

This controller was analogous to classical proportional-derivative control of torque, with damping injection $[44,100]$ on motor velocity taking the place of the derivative term:

$$
\Delta \theta_{p, d e s}=-K_{p} \cdot e_{\tau}-K_{d} \cdot \dot{\theta}_{p}
$$

where $K_{p}$ is a proportional gain, $e \tau=\tau-\tau_{d e s}$ is torque error, $\tau$ is measured exoskeleton torque, $\tau_{d e s}$ is desired exoskeleton torque, $K_{d}$ is a damping gain, and $\dot{\theta}_{p}$ is measured velocity of the motor pulley. In pilot testing, we found the damping term more effective than a term with the derivative of torque error; torque was measured with analog strain gauges, which included substantial noise, while pulley position was measured with a digital encoder. Damping was placed on motor pulley velocity alone rather than the relative velocity between the motor pulley and the exoskeleton joint. In pilot tests, using relative velocity was less effective, likely due to the irregular effects of stiction in the Bowden cable transmission on ankle joint velocity.

$L^{2:}$ Proportional Control with Damping Injection and Error-Dependent Gains ( $\left.\mathrm{PD}^{*}+\mathrm{EDG}\right)$ 
This controller was identical to $L^{1}$, with the exception that the proportional gain was error-dependent [40, 41], and increased with torque error:

$$
\begin{aligned}
K_{p}^{*} & =\min \left(\left\lceil\frac{\left|e_{\tau}\right|}{h_{\tau}}\right\rceil \cdot h_{k}, K_{\max }\right) \\
\Delta \theta_{p, \text { des }} & =-K_{p}^{*} \cdot e_{\tau}-K_{d} \cdot \dot{\theta}_{p}
\end{aligned}
$$

where the symbol $\lceil\cdot\rceil$ denotes the ceiling operation, $K_{p}^{*}$ is the error-dependent proportional gain, $h_{\tau}$ and $h_{k}$ are torque error and proportional gain step sizes, and $K_{\max }$ is the maximum allowable gain. This is similar to performing proportional control on the square of the torque error, with a sign and gain adjustment. This type of gain scheduling is expected to result in slower corrections, and fewer oscillations, when torque tracking errors are small.

$L^{3:}$ Proportional Control with Damping Injection and Previous-Error Compensation $\left(\mathrm{PD}^{*}+\mathrm{PEC}\right)$

This controller was identical to $L$, except that desired torque was altered based on torque error from the previous instant in time $[126,127]$ as:

$$
\begin{aligned}
\tau_{d e s}^{\prime} & =\tau_{d e s}-e_{\tau, p r e v} \\
\Delta \theta_{p, d e s} & =-K_{p e c} \cdot\left(\tau-\tau_{d e s}^{\prime}\right)-K_{d} \cdot \dot{\theta}_{p}
\end{aligned}
$$

where $\tau_{d e s}^{\prime}$ is the compensated torque error and $e_{\tau}$,prev is the torque error from the previous time step. $K_{p e c}$ is a proportional gain. This approach is expected to increase the control response to large errors. It bears some similarity to integral control, in that it includes a term on prior error, but differs in that only the prior error at the previous sampling time is used rather than the entire time history. In cases where torque error changes slowly, this approach equates to doubling the proportional gain. 
$L^{4:}$ Proportional-Integral Control with Damping Injection (PID*)

This controller was analogous to classical proportional-integral-derivative control, with damping injection substituted for the derivative term:

$$
\Delta \theta_{p, d e s}=-K_{p} \cdot e_{\tau}-K_{i} \cdot \int_{t_{0}}^{t} e_{\tau} d t-K_{d} \cdot \dot{\theta}_{p}
$$

where $K_{i}$ is the gain on the integral of torque error, $t_{0}$ is the time at which the stride began, and $t$ is the present time. Integral control is expected to eliminate steady-state error by accumulation of control input $[83,84,95]$.

\section{Model-Based Feed-Forward Control}

Many systems with series elastic actuators use an inverse dynamics model of the series spring as a feed-forward control element, typically added to a model-free feedback component. We implemented one such model-based controller in this study, ${ }_{L}^{5}$.

$L^{5:}$ Proportional Control with Damping Injection and Model-based Compensation $\left(\mathrm{PD}^{*}+\mathrm{M}\right)$

This controller included both the classical feedback controller of $L^{1}$ and a modelbased feedforward term, which was intended to anticipate changes in desired motor position due to either changes in exoskeleton joint angle or changes in desired joint torque:

$$
\begin{aligned}
\Delta \theta_{p, d e s} & =-K_{p} \cdot e_{\tau}-K_{d} \cdot \dot{\theta}_{p}+\left(\theta_{m d l}-\theta_{p}\right) \\
\theta_{m d l} & =\theta_{e} \cdot \tilde{R}-\tau_{d e s} \cdot \tilde{K}_{t}^{-1}
\end{aligned}
$$

where $\theta_{m d l}$ is a model-based motor position compensation generated from Eq. (4.6), 
$\theta_{p}$ is measured motor pulley position, $\theta_{e}$ is measured exoskeleton ankle joint angle, $\tilde{R}$ is the estimate of $R$ as defined in Eq. (5.3), or the ratio of the exoskeleton lever arm to the motor pulley radius, and $\tilde{K}_{t}$ is an estimate of $K_{t}$, which is the total stiffness of the tether, series spring, and other structures between the motor pulley and exoskeleton joint as defined by Eq. (5.5). This is an inverse dynamics approach similar to computed torque and feedback linearization in nonlinear control. If desired torque remains constant but the exoskeleton joint moves, we expect the motor to need to move in proportion. If the joint is stationary but desired torque changes, we expect we know how much to move the motor to obtain the desired change in torque.

We also performed pilot tests with a version of this controller in which change in pulley angle, rather than absolute pulley angle, was anticipated based on the rate of change in exoskeleton angle and the rate of change in desired torque. This approach was less stable, owing to the effects of Bowden cable stiction on exoskeleton joint angle and the interplay between user behavior and desired torque through the high-level controller.

\section{Model-Based Feedback Control}

Adaptive control approaches $[45,34]$ using more complete system models have also been applied to exoskeletons. Such regimes have the capacity to exploit additional knowledge of system dynamics and allow theoretical tests of stability and performance. We developed a new adaptive controller for this system using a passivity-based approach, ${ }_{L}^{6}$. 
Combining the dynamics of the subsystems described by Eqs. (4.1)-(4.8) and eliminating $F$ and $i_{a}$, we have following dynamics of the system:

$$
\ddot{\tau}+K_{1} \dot{\tau}+K_{2} \tau+S_{\theta} \dot{\theta}_{e}=K_{V} V_{a}+K_{h} \tau_{h}
$$

in which $\tau$ denotes the torque transmitted to the exoskeleton from the motor, $\theta_{e}$ denotes the exoskeleton joint angle, $V_{a}$ is the voltage applied to the armature of the motor, and $\tau_{h}$ denotes the torque applied to exoskeleton by human body. $K_{V}$, $K_{1}, K_{2}$ and $K_{h}$ are positive gains expressed as

$$
\begin{aligned}
K_{V} & =\frac{r_{a} r_{p} K_{a} K_{c}}{I_{e} N R_{a}} \\
K_{1} & =\frac{1}{I_{e}}\left(\frac{K_{a} K_{b}}{R_{a}}+f_{e}\right) \\
K_{2} & =\frac{r_{p}^{2} K_{c}}{N^{2} I_{e}}+\frac{r_{a}^{2} K_{c}}{I_{e}} \\
K_{h} & =\frac{r_{a}^{2} K_{c}}{I_{e}}
\end{aligned}
$$

and $S_{\theta}$ is a gain expressed as

$$
S_{\theta}=\left[\frac{r_{a}^{2} K_{c}}{I_{e}}\left(\frac{K_{a} K_{b}}{R_{a}}+f_{e}\right)-\frac{r_{a}^{2} K_{c} B_{e}}{I_{e}}\right]
$$

with definitions of constants provided in Section 5.2.1. Based on the system model in Eq. (4.18), we developed a new, provably stable, adaptive controller for the system.

We define a controller as:

$$
\begin{aligned}
V_{a}= & -K_{p} \cdot e_{\tau}-K_{s} \cdot s \\
& +Y_{d}\left(\tau, \dot{\tau}_{r}, \ddot{\tau}_{r}, \dot{\theta}_{e}\right) \cdot \tilde{\Gamma} \\
& -K_{s w} \cdot \operatorname{sign}(s)
\end{aligned}
$$


where $K_{p}$ and $e_{\tau}$ are as defined in ${ }_{L} 1, K_{S}$ is the sliding control gain, $s$ is the sliding vector, defined below, $Y_{d}$ is a regressor, defined below, $\Gamma$ and $\tilde{\Gamma}$ are the system parameter vector and its estimate, defined below, and $K_{S w}$ is the switching term gain. The sliding vector $s$ is defined as:

$$
s=\dot{\tau}-\dot{\tau}_{d e s}+\lambda \cdot e_{\tau}=\dot{\tau}-\dot{\tau}_{r}
$$

where $\lambda$ is a positive scalar and $\tau_{r}$ is a virtual reference torque. The regressor, $Y_{d}$, is defined as:

$$
Y_{d}\left(\tau, \dot{\tau}_{r}, \ddot{\tau}_{r}, \dot{\theta}_{e}\right)=\left[\begin{array}{llll}
\tau & \dot{\tau}_{r} & \ddot{\tau}_{r} & \dot{\theta}_{e}
\end{array}\right]
$$

and is used to express the dynamics as linear combination of system parameters as:

$$
Y_{d} \cdot \Gamma=\frac{1}{K_{v}} \cdot \ddot{\tau}_{r}+\frac{K_{1}}{K_{v}} \cdot \dot{\tau}_{r}+\frac{K_{2}}{K_{v}} \cdot \tau+\frac{S_{\theta}}{K_{v}} \cdot \dot{\theta}_{e}
$$

The system parameter, $\Gamma$, is defined as:

$$
\Gamma=K_{v}^{-1} \cdot\left[\begin{array}{llll}
1 & K_{1} & K_{2} & S_{\theta}
\end{array}\right]^{\top}
$$

With full knowledge of system parameters, or $\tilde{\Gamma}=\Gamma$, Eq. (4.19) describes a model-based computed torque controller. For practical reasons, however, it is difficult to identify the value of $\Gamma$. Therefore, an update law is added to estimate the system parameters, $\tilde{\Gamma}$, as follows:

$$
\dot{\tilde{\Gamma}}=-L Y_{d}^{\top} s
$$

where $\mathrm{L}$ is a symmetric positive definite parameter adaptation gain matrix. This parameter updating process reduces the model-dependency of controller in Eq. (4.19), because only the structure of the dynamic model is used in the construction of controller. 
The closed-loop system with the model-based adaptive controller described by Eqs. (4.19)-(4.20) and dynamics described by Eq. (4.18) is stable and the exoskeleton torque trajectory $\tau$ converges to the desired value of $\tau_{d e s}$, provided that the human input torque, $\tau_{h}$, the desired torque trajectory, $\tau_{d e s}$, and their time derivatives, $\dot{\tau}_{h}, \dot{\tau}_{d e s}$, and $\ddot{\tau}_{d e s}$, are bounded. A proof is provided in Appendix A. In pilot tests, we found that better performance was obtained with this controller by setting the time rate of change in desired torque to zero. In practice, for most high-level controllers, the time derivative of desired torque, $\dot{\tau}_{d e s}$, could not be calculated in advance and contained substantial noise when approximated numerically. Noise on this signal arose from the human measurements used by the high-level controllers to calculate desired torque. We also found that the time derivative of torque error, $\dot{e} \tau$, contained substantial noise, in part due to noise on the analog strain gauge signal and in part due to complex Bowden cable transmission dynamics. Better performance was obtained using motor output pulley velocity, $\dot{\theta}_{p}$, in its place. This substitution is equivalent to assuming that the characteristic time of exoskeleton joint dynamics was much larger than the characteristic time of motor dynamics (Eq. 4.6). It is also analogous to the use of damping injection in place of derivative control in the other controllers tested. The sliding vector and regressor are therefore approximated as:

$$
\begin{aligned}
s & \approx \dot{\theta}_{p}+\lambda \cdot e_{\tau} \\
Y_{d} & \approx\left[\begin{array}{lll}
\tau-\lambda \cdot e_{\tau}-\lambda \cdot \dot{\theta}_{p} & \dot{\theta}_{e}
\end{array}\right]
\end{aligned}
$$

Additionally, in pilot tests we found that it was more effective to operate the dedicated motor drive in velocity control mode, rather than voltage control mode. This difference in performance is likely due to the faster control loop in the motor driver, which allowed voltage to be changed more frequently and with less delay than for the control system as a whole. Motor velocity is strongly related to applied voltage, since the two are linearly related for a given torque at steady state 
(Eq. 4.11). This led to a similar formulation as for all other low-level controllers:

$$
\begin{aligned}
\Delta \theta_{p, d e s}= & -K_{p} \cdot e_{\tau}-K_{S} \cdot s \\
& +Y_{d}\left(\tau, e_{\tau}, \dot{\theta}_{p}, \dot{\theta}_{e}\right) \cdot \tilde{\Gamma} \\
& -K_{s w} \cdot \operatorname{sign}(s)
\end{aligned}
$$

\section{Model-Free Feed-Forward Control}

We also tested a group of controllers that use iterative learning as a feed-forward component, which were expected to improve performance by exploiting the cyclic nature of human gait.

\section{${ }^{7}$ 7: Iterative Learning of Desired Motor Position (LRN)}

This controller used torque error at each instant of one stride to update a feedforward trajectory of desired motor position for each instant of the next stride. This is a variation on iterative learning, which, more generally, exploits the cyclic nature of a task to compensate complex system dynamics without an explicit model [37, 128-130]. While walking is not as consistent as the operations of most industrial robots, it is cyclic, which was expected to afford some improvement in torque errors that occurred consistently from stride to stride.

The feed-forward trajectory of desired motor position, $\theta_{p, d e s}$, was calculated as:

$$
\theta_{p, d e s}(i, n+1)=\theta_{p, d e s}(i, n)-K_{l} \cdot e_{\tau}(i, n)
$$

where $i$ is the time index or number of control cycles elapsed within this stride, $n$ is this stride and $n+1$ is the next stride, and $K_{l}$ is the iterative learning gain. Desired motor position was then enforced as:

$$
\Delta \theta_{p, d e s}(i, n)=\theta_{p, d e s}(i+D, n)-\theta_{p}(i, n)
$$


where $D$ is an estimate of the delay between commanding and achieving a change in motor position. During tuning, both $K_{l}$ and $D$ were adjusted.

Current torque error thereby updates desired motor position for the same time index on the next stride, while commanded motor velocity at this time index is based on a preview of desired motor position later in the same stride. Since the learned trajectory used in the present step has no dependence on the present torque, this method is feed-forward. However, present motor pulley position measurements were used in generating present motor velocity commands. This method can therefore be viewed as a feed-forward iterative learning control of torque combined with proportional feedback control of motor position.

\section{Forgetting during Learning}

To avoid divergence due to excessive accumulation of ripples during the learning process, a 'forgetting' constant was introduced to Eq. (4.22) as:

$$
\theta_{p, d e s}(i, n+1)=\beta \cdot \theta_{p, d e s}(i, n)-K_{l} \cdot e_{\tau}(i, n)
$$

where $\beta \in[0,1]$ is a weight on the learned trajectory. For $\beta=1$, all learning is retained, zero steady-state offset is expected, but ripples can form if the value of $D$ is incorrect. For $\beta<1$, torque errors from strides before the last stride have a reduced effect on controller behavior, reducing likelihood of ripple formation, but leading to some steady-state torque offset. For $\beta=0$, iterative learning is disabled. $L^{7}$ then becomes proportional control based on the torque error delayed by one step, and poor torque tracking performance is expected.

\section{Learning from Filtered Errors}

Noise in the error signal leads to inappropriate updates on the learned trajectory, which can excite unstable ripple formation. This excitation can be reduced by 
filtering the error signal across strides:

$$
e_{f l t}(i, n)=(1-\mu) \cdot e_{f l t}(i, n-1)+\mu \cdot e_{\tau}(i, n)
$$

where $e_{f l t}$ is the filtered torque error trajectory, initially an array of zeros, used in place of $e \tau$ in Eq. (4.22) and (4.24), and $\mu \in[0,1]$ is a weighting term on the learned error. For $\mu=1$, only the error from the last stride is used to update the motor trajectory, resulting in faster convergence but larger effects of sensor noise. For $\mu<1$, errors at this time increment from all prior strides have some effect on the motor trajectory update, resulting in slower but more stable convergence. For $\mu=0$, torque error is not updated, and iterative learning is disabled.

$L^{8:}$ Iterative Learning of Desired Motor Position + Proportional-Damping Compensation $\left(\mathrm{LRN}+\mathrm{PD}^{*}\right)$

This controller combined iterative learning with proportional-damping feedback control to compensate remaining torque errors. It is a direct superposition of controllers $L^{1}$ and $L^{7}$, in which the absolute desired motor position was learned as in $L^{7}$ and feedback control was applied as in $L^{1}$ :

$$
\begin{aligned}
\theta_{p, d e s}^{L R N}(i, n+1)= & \beta \cdot \theta_{p, d e s}^{L R N}(i, n)-K_{l} \cdot e_{f l t}(i, n) \\
\theta_{p, \text { des }}(i, n)= & \theta_{p, d e s}^{L R N}(i+D, n) \\
& -K_{p} \cdot e_{\tau}(i, n)-K_{d} \cdot \dot{\theta}_{p}(i, n) \\
\Delta \theta_{p, \text { des }}(i, n)= & \theta_{p, \text { des }}(i, n)-\theta_{p}(i, n)
\end{aligned}
$$

Combining iterative learning with feedback control is thought to result in improved performance compared to either component used in isolation [92]. Iterative learning is expected to generate a feed-forward trajectory that tracks torque for an average stride with zero stead-state error regardless of the complexity of 
the command signal required, but to be susceptible to step-by-step variability. Proportional-damping control is expected to quickly compensate for small torque errors, but to be susceptible to rapid changes in desired or measured torque.

$L^{9:}$ Proportional Control with Damping Injection + Iterative Learning Compensation $\left(\mathrm{PD}^{*}+\Delta \mathrm{LRN}\right)$

This controller is another combination of proportional-damping feedback and iterative learning feed-forward control elements. Unlike controllers $L^{7}$ and $L^{8}$, the values to be learned are desired changes in motor position instead of absolute desired positions:

$$
\begin{aligned}
\Delta \theta_{p, d e s}^{L R N}(i, n+1)= & \beta \cdot \Delta \theta_{p, d e s}^{L R N}(i, n)-K_{l} \cdot e_{f l t}(i, n) \\
\Delta \theta_{p, d e s}(i, n)= & -K_{p} \cdot e_{\tau}(i, n)-K_{d} \dot{\theta}_{p}(i, n) \\
& +\Delta \theta_{p, d e s}^{L R N}(i+D, n)
\end{aligned}
$$

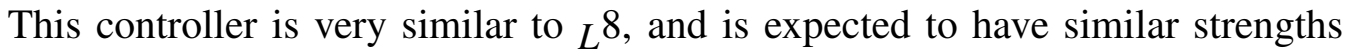
and weaknesses. Differences in motor position are learned rather than absolute positions, however, which eliminates measured motor pulley position, $\theta_{p}$, from the formulation. It is therefore a velocity control approach rather than a position control approach. This may affect stability, drift and the level and source of noise in the learned trajectory, which may in turn affect the allowable gains and speed of convergence. Learning desired changes in position also affects interactions between the feedback and feed-forward elements of the controller in the presence of step-by-step variability; learned changes in position add the same way regardless of present position and error, while the contribution of learned absolute positions depends upon the present motor position. Either approach can oppose feedback contributions under some conditions, but in different ways. A detailed mathematical comparison of these two approaches is provided in Appendix C. 


\section{Additional Feedback Control Terms Piloted}

Several control elements that seemed likely to improve performance in theory did not fare well in pilot tests. This may be due to the unique features of the control problem at hand, in particular the noisy sensory information and the complex, changing dynamics of both the Bowden cable transmission and the human. These approaches were not included in the final data collection.

One such example is the traditional derivative control element

$$
-K_{d}\left(\dot{\tau}-\dot{\tau}_{d e s}\right)
$$

which involves the derivative of torque error. Analog noise in the derivative of measured torque limited the magnitude of the derivative gain that could be applied without causing oscillations. This limited the capacity of the derivative term to stabilize the system, in turn limiting the magnitude of the proportional gains that could be applied.

Using the model described by Eq. (4.6), we next approximated the derivative term as:

$$
-K_{d}\left[\left(\dot{\theta}_{p}-\dot{\theta}_{e} R\right)-\dot{\tau}_{d e s} \cdot \tilde{K}_{t}\right]
$$

where the relative velocity between the motor and exoskeleton was substituted for the noisy measured torque derivative. The derivative of desired torque is also problematic, however, because it generally cannot be calculated in advance and its numerical approximation online is subject to noise from the human measurements used by the high-level controller to calculate desired torque, for example electromyographic measurements.

We next tried using just the relative velocity between the motor pulley and exoskeleton joint:

$$
-K_{d}\left(\dot{\theta}_{p}-\dot{\theta}_{e} R\right)
$$


which is equivalent to making the additional approximation that the derivative of desired torque, $\dot{\tau}_{d e s}$, is negligible. However, this control element was also found to be ineffective in pilot tests due to noise on the derivative of the exoskeleton joint angle, which seems to primarily arise from stiction in the Bowden cable transmission.

Finally, we arrived at the simple damping term

$$
-K_{d} \dot{\theta}_{p}
$$

which relied only upon the derivative of motor pulley position, which had little noise to amplify, and provided sufficient damping to improve stability.

We also pilot tested proportional control without a damping term, which was effective. However, the addition of some damping always allowed for higher proportional gains and improved tracking performance. Therefore, proportional control was always used together with damping injection in our tests.

\section{High-Level Assistance Controllers}

\section{Stance Torque Control}

During the stance period, desired exoskeleton joint torque was set according to one of four high-level assistance controllers, $H^{1-} H^{4}$, described below.

$H^{1:}$ Time Based Desired Torque Trajectory (TIME)

This high-level controller set desired torque as a function of time. Time-based control elements are simple and easily understood, and have been incorporated 


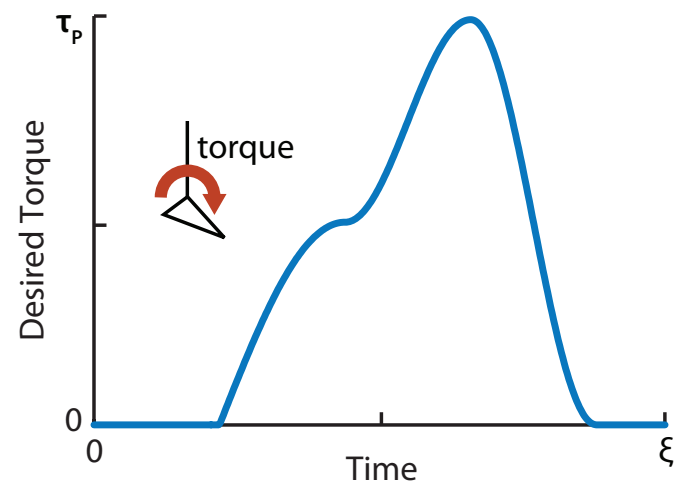

Figure 4.5: High-level control based on a trajectory in time

into many exoskeleton systems $[107,131,30,106]$. We used a curve that resembled a scaled-down version of the human ankle moment during unassisted walking, calculated as:

$$
\begin{aligned}
\tau_{d e s}= & \\
0<t<0.15 \xi: & 0, \\
0.15 \xi<t<0.30 \xi: & \frac{\tau_{p}}{2} \sin \left(\frac{t-0.15 \xi}{0.3 \xi} \pi\right), \\
0.30 \xi<t<0.45 \xi: & \frac{-\tau_{p}}{4} \cos \left(\frac{t-0.3 \xi}{0.15 \xi} \pi\right)+\frac{3 \tau_{p}}{4} \\
0.45 \xi<t<0.60 \xi: & \frac{\tau_{p}}{2} \cos \left(\frac{t-0.45 \xi}{0.15 \xi} \pi\right)+\frac{\tau_{p}}{2}, \\
0.60 \xi \leq t \quad: & 0,
\end{aligned}
$$

where $t$ is the time since the current stride began, $\xi$ is stride period and $\tau_{p}$ is peak torque. We used $\xi=1.1 \mathrm{~s}$ and $\tau_{p}=45 \mathrm{~N} \cdot \mathrm{m}$ in this experiment, which produced the desired torque profile shown in Fig. 4.5.

\section{$H^{2}$ : Joint Angle Based Desired Torque (ANGLE)}

This high-level controller set desired torque as a function of exoskeleton ankle joint angle and phase of the gait cycle. This approach is a subset of impedance control, and is similar to setting desired torque based on a phase variable rather than clock time. Variations have been employed in many assistive devices [132, 


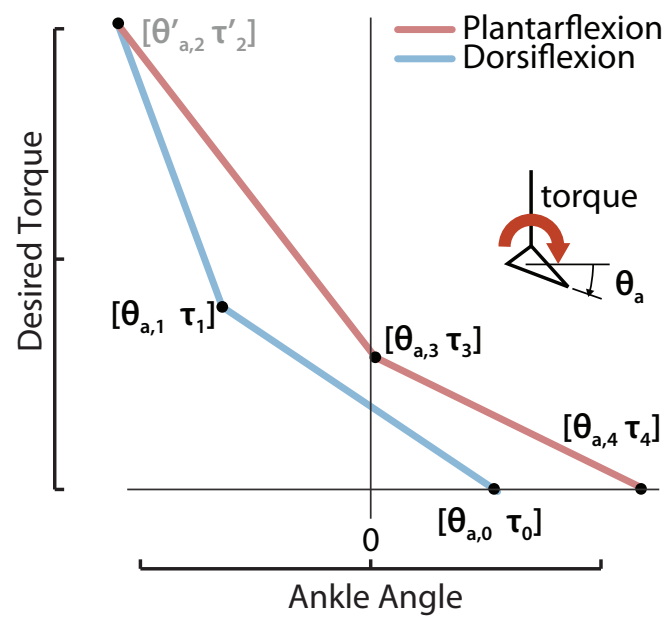

Figure 4.6: High-level control based on ankle joint angle

$78,89]$. We used a piece-wise linear curve that resembled a scaled-down version of the human ankle moment during unassisted walking, calculated as:

$$
\tau_{d e s}=\frac{\tau_{i}-\tau_{i-1}}{\theta_{e, i}-\theta_{e, i-1}}\left(\theta_{e}-\theta_{e, i-1}\right), \quad i=\{1,2,3,4\}
$$

with curve parameter values as listed in Table 6.2.

Table 4.1: Angle-based control parameter values

\begin{tabular}{llll}
\hline Param & Value & Param & Value \\
\hline$\left[\theta_{0}, \tau_{0}\right]$ & {$[0.018,0.00]$} & {$\left[\theta_{3}, \tau_{3}\right]$} & {$[0.00,11.3]$} \\
{$\left[\theta_{1}, \tau_{1}\right]$} & {$[-0.122,18.1]$} & {$\left[\theta_{4}, \tau_{4}\right]$} & {$[0.140,0.00]$} \\
{$\left[\theta_{2}, \tau_{2}\right]$} & {$[-0.209,45.2]$} & & \\
\hline
\end{tabular}

Here, $\left(\theta_{i}, \tau_{i}\right)$ defines a node in torque-angle space (Fig. 6.1). The node $\left(\theta_{2}, \tau_{2}\right)$ marked the transition from the dorsiflexion phase, in which ankle velocity was negative, to the plantarflexion phase, in which ankle velocity was positive. Since the exact transition point varied on each stride, we used the angle and torque at the moment of transition, $\left(\theta_{2}^{\prime}, \tau_{2}^{\prime}\right)$, when calculating desired torque in the first portion of Plantarflexion.

$H^{3:}$ Neuromuscular Model Based Desired Torque (NMM)

This high-level controller set desired exoskeleton torque based on a Hill-type 


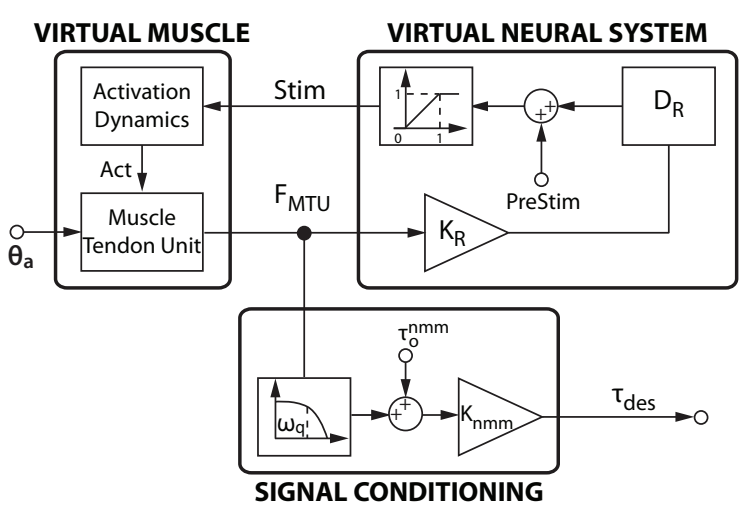

Figure 4.7: Neuromuscular model control schematic

muscle model and a positive force feedback reflex model. The resulting dynamics produce human-like motions and muscle activation patterns in simulation [133] and are thought to interact well with the human neuromuscular system [112-116]. Virtual muscle-tendon-unit length and velocity were set by measured exoskeleton joint angle and angular velocity. Virtual fiber length, velocity and activation were then used to determine muscle-tendon-unit force, $F_{\mathrm{mtu}}$, which, after conditioning, was used to set desired exoskeleton torque. We conditioned the force signal by applying a low-pass filter with frequency $\omega_{q}$, adding a small negative offset of $\tau_{O}^{n m m}$, and applying a gain of $K_{n m m}$. Virtual muscle force was also used to drive a positive force feedback loop in which increased force led to increased muscle activation. The virtual neural system multiplied muscle force by a reflex gain, $K_{\mathrm{R}}$, applied a time delay of $D_{\mathrm{R}}$, added a small positive offset, PreStim, then applied a threshold, yielding the virtual muscle stimulation. Virtual muscle activation was driven by stimulation through first-order dynamics. A high-level schematic is provided in Fig. 4.7, high-level parameters are found in Table 4.2, and a full set of equations and parameters are available in Appendix B.

\section{$H^{4:}$ Electromyography Based Desired Torque (EMG)}

This high-level controller set desired torque in proportion to electromyographic measurements from the human gastrocnemius muscle. This approach gives the 
Table 4.2: NMM parameter values

\begin{tabular}{llll}
\hline Param & Value & Param & Value \\
\hline$K_{\mathrm{R}}$ & 0.002 & $\omega_{q}$ & $50 \mathrm{~Hz}$ \\
$D_{\mathrm{R}}$ & $0.020 \mathrm{~s}$ & $\tau_{\mathrm{O}}^{n m m}$ & -20 \\
PreStim & 0.05 & $K_{n m m}$ & $0.057 \mathrm{~N} \cdot \mathrm{m}$ \\
\hline
\end{tabular}

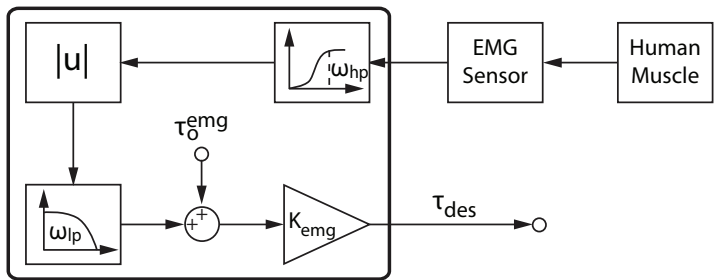

SIGNAL CONDITIONING

Figure 4.8: Proportional electromyography control schematic

user direct neural control of the device, which is intended to make interactions more intuitive $[20,119,120]$, but can result in more complex desired torque dynamics. Electrical activity in the gastrocnemius was measured using surface electrodes and a commercial electromyography system. The signal was then highpass filtered at a frequency of $\omega_{h p}$, rectified, and low-pass filtered at a frequency of $\omega_{l p}$. A small negative offset, $\tau_{o}^{e m g}$, was applied, which prevented desired torque generation at low levels of muscle activity. The signal was then amplified by a gain, $K_{e m g}$, yielding desired torque. A high-level schematic is provided in Fig. 4.8, and the parameters used in this experiment can be found in Table 4.3.

Table 4.3: EMG parameter values

\begin{tabular}{llll}
\hline Param & Value & Param & Value \\
\hline$F_{h p}$ & $20 \mathrm{~Hz}$ & $F_{l p}$ & $6 \mathrm{~Hz}$ \\
$K_{\text {emg }}$ & 283 & $\tau_{o}^{\text {emg }}$ & -0.008 \\
\hline
\end{tabular}

\section{Swing Control}

When the foot was off the ground, motor position control was employed to allow 
free motion of the human ankle and maintain a small amount of slack in the cable:

$$
\begin{aligned}
\theta_{p, d e s} & =\theta_{e} \cdot \tilde{R} \\
\Delta \theta_{p, d e s} & =\theta_{p, d e s}-\theta_{p}
\end{aligned}
$$

where $\theta_{e}$ is exoskeleton joint angle and $\tilde{R}$ is the estimated total gear ratio from motor to exoskeleton joint. Maintaining low slack in the Bowden cable reduced the time required for cable winding at the beginning of stance.

\subsubsection{Experimental Methods}

All experiments were conducted with one $(\mathrm{N}=1)$ healthy adult participant (30 yrs, $56 \mathrm{~kg}, 1.65 \mathrm{~m}$ tall, female). Multiple participants were not warranted in the main experiments of this study, as the main objective was to examine torque tracking performance by the exoskeleton, and most torque controllers were developed independent of the human body dynamics. The participant walked on a treadmill at $1.25 \mathrm{~m} \cdot \mathrm{s}^{-1}$ with a self-selected stride period of $1.08 \pm 0.06 \mathrm{~s}$ while wearing the exoskeleton on one leg. The participant provided written informed consent prior to participation in the study, which was conducted in accordance with a protocol approved by the Carnegie Mellon University Institutional Review Board.

Before collecting data, we tuned parameters for each combination of high- and lowlevel controller as the participant walked with the exoskeleton. High-level control parameters listed in the prior section were selected so as to result in peak instantaneous desired torques of approximately $45 \mathrm{~N} \cdot \mathrm{m}$ during the course of one hundred steps of walking. Low-level control parameters listed in Table 4.4 were systematically tuned with the aim of minimizing torque error. Feedback, model and adaptive control gains in ${ }_{L}^{1-}{ }_{L} 6$ and ${ }_{L} 8-{ }_{L} 9$ were tuned using a variant of the Ziegler-Nichols method [134], in which: 
1. All gains (proportional, damping, integral, model, sliding, and/or adaptive) were set to zero.

2. The proportional gain was increased until significant oscillations were observed.

3. Gain value and oscillation period were then recorded and used to estimate optimal values for proportional and damping gains.

4. Fine tuning of gains for all control elements, other than iterative learning, was then performed by the experimenter.

The iterative learning gain in $L^{7}$ was tuned such that steady state was reached at approximately 10 strides, which led to a value of $K_{l}$ that was about one tenth the tuned value of $K_{p}$. The same gains were used for iterative learning elements in controllers $L^{7}-L^{9}$. During tuning we found very similar optimal low-level control parameters across high-level controllers, and so used identical values within each low-level controller for consistency. Tuning was performed on a separate day from data collection. For model-based compensation, the value of $\tilde{R}$ was based on measurements of the motor output pulley radius, motor gear ratio, and exoskeleton lever arm. $\tilde{K}_{c}$ was estimated based on measurement of the passive relationship between exoskeleton torque and exoskeleton joint angle measured during walking experiments (Fig. 6.3).

Table 4.4: Low-level torque control parameter values (Note: $I_{3}$ denotes a $3 \times 3$ identity matrix)

\begin{tabular}{llllll}
\hline \multicolumn{2}{l}{ Param Value } & \multicolumn{2}{l}{ Param Value } & \multicolumn{2}{c}{ Param Value } \\
\hline$K_{p}$ & 0.093 & $\tilde{R}$ & 2.90 & $K_{l}$ & 0.0077 \\
$K_{d}$ & 0.010 & $\tilde{K}_{c}$ & $195 \mathrm{~N} \cdot \mathrm{m} \cdot \mathrm{rad}^{-1}$ & $D$ & $0.022 \mathrm{~s}$ \\
$K_{\max }$ & 0.15 & $K_{S}$ & 0.005 & $\beta$ & 1 \\
$K_{\text {pec }}$ & 0.046 & $\lambda$ & 0.077 & $\mu$ & 1 \\
$K_{i}$ & $7.7 \mathrm{e}-5$ & $L$ & $1.0 \mathrm{e}-9 I_{3}$ & $T$ & $0.250 \mathrm{~s}$ \\
$h_{\tau}$ & $11.3 \mathrm{~N} \cdot \mathrm{m}$ & $h_{k}$ & 0.039 & $K_{S w}$ & 0 \\
\hline
\end{tabular}

For each high-level controller, all low-level control conditions were tested on the same day, without removal of the exoskeleton between trials. A table of condition 
order is presented in Supporting Materials Table SI.

For each combination of low-level torque control and high-level assistance control, we collected data from 100 steady-state strides. Steady state was verified by visual inspection, and was typically reached after about 20 strides. The subsequent 100 strides were then decomposed into individual strides, each beginning at heel strike as detected by a shoe-embedded switch. Data for an average stride were then calculated by taking the mean across all strides at each instant in time.

For each condition, we calculated torque error both for the set of all steady-state strides and for the average stride. We quantified torque error as the root mean squared error of the difference between measured and desired torque. For the set of all steadystate strides, we calculated root mean squared error for each stride individually, then calculated the mean and standard deviation. For the set of all steady-state strides, we compared means within high-level controllers and across low-level controllers using unpaired t-tests, with a significance level of $\alpha=0.05$.

\subsection{Results}

Means and standard deviations of stride-wise root-mean-squared torque error (RMSE) and average-stride root-mean-squared error (RMS-E AVG) of all low- and highlevel controller combinations are shown in Fig. 4.9. A complete table of $p$ values for statistical comparisons between the RMS-E of all torque controllers are provided as Supporting Materials (Tables SII-SV). Overlapped time trajectories of desired and measured joint torques across all one hundred steady-state strides in each condition are shown in Fig. 4.10. Ankle angle trajectories in time and torque trajectories in ankle angle space are also provided for all conditions as Supporting Materials (Figs. S1S2). Convergence plots for controllers that involved iterative learning are provided as 


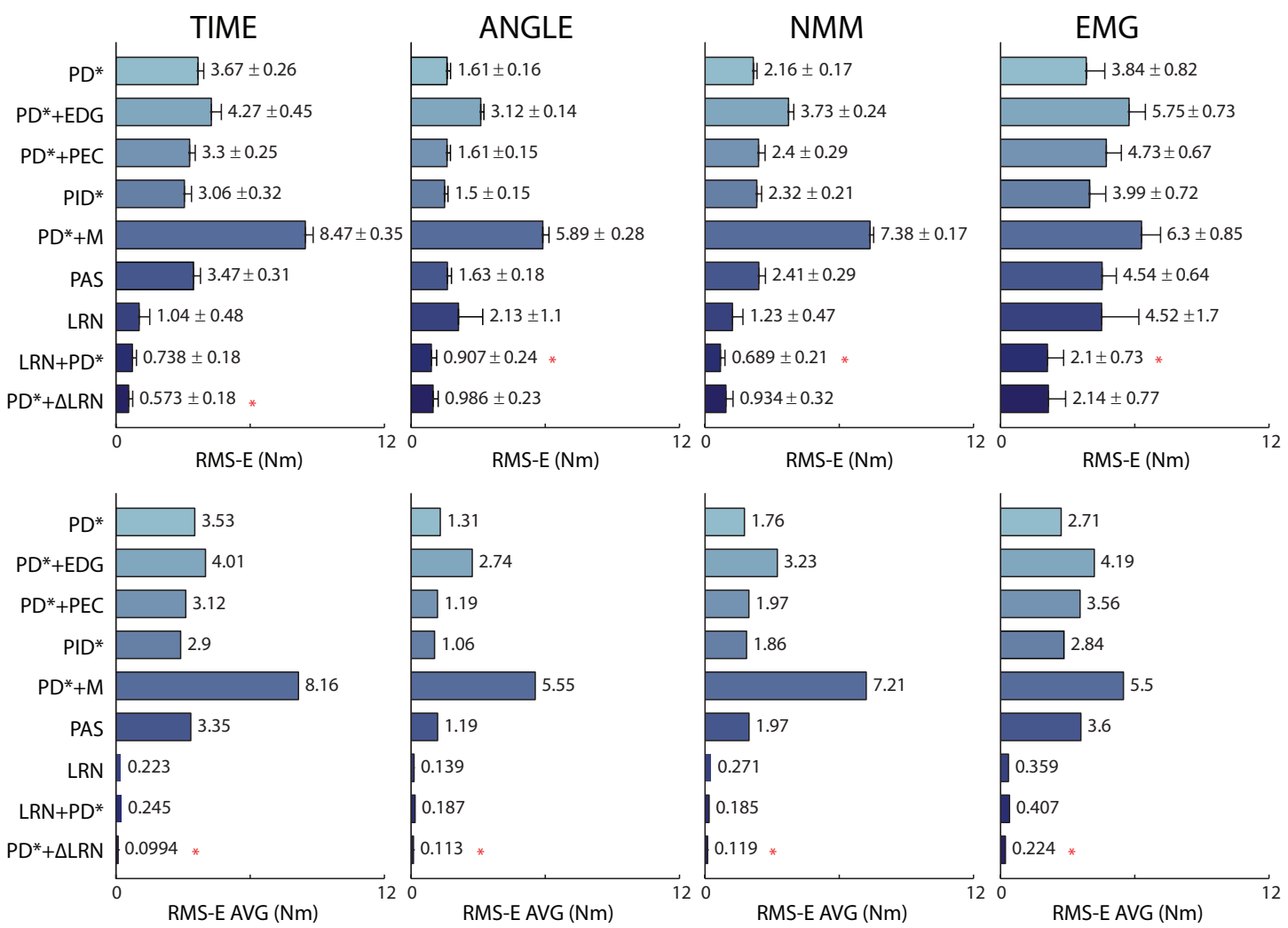

Figure 4.9: Root-mean-squared torque error calculated for all strides (RMS-E) and for an average stride (RMS-E AVG) across all high- and low-level control combinations.

Supporting Materials (Fig. S4).

The combination of proportional control and damping injection with iterative learning $\left(\mathrm{PD}^{*}+\Delta \mathrm{LRN}\right.$ or $\left.\mathrm{LRN}+\mathrm{PD}^{*}\right)$ resulted in the lowest torque tracking errors for all high-level controllers, both in real-time and for average trajectories (Fig. 4.9). Of these two combinations with comparable performance, feedback control with learning compensation $\left(\mathrm{PD}^{*}+\Delta \mathrm{LRN}\right)$ was simpler and converged faster. Stride-wise torque errors with $\mathrm{PD}^{*}+\Delta \mathrm{LRN}$ were between $38 \%$ and $84 \%$ lower than with $\mathrm{PD}^{*}$ alone $\left(\mathrm{p}<1.9 \cdot 10^{-43}\right)$, while average-stride torque errors were between $91 \%$ and $97 \%$ lower, depending on high-level controller. Iterative learning control alone tended to result in low errors for average trajectories, but higher real-time errors than when combined with feedback control. Other additions to feedback control had minor effects on performance, except for model-based compensation, which increased torque error substantially. When desired torque was based on EMG, torque tracking error and variability were higher for 
almost all torque controllers. Values for the $\mathrm{PD}^{*}+\Delta \mathrm{LRN}$ controller, including errors as a percentage of the maximum of the average desired torque, are provided in Table 4.5. The contributions of each component of the $\mathrm{PD}^{*}+\Delta \mathrm{LRN}$ controller to desired motor displacement, and their evolution in time, are depicted in Fig. 4.11.

There were some interactions between high-level control type and low-level torque control performance. With Angle and EMG based high-level controllers, pure feedback control was more effective than pure iterative learning control, while for Time and NMM based controllers this trend was reversed. With Time-based desired torque, all controllers that did not have a learning component had poor tracking at the onset of desired torque, including a delay and overshoot, that comprised a large portion of the total torque error (Fig. 4.10). The addition of iterative learning to $\mathrm{PD}^{*}$ control led to the greatest reductions in torque errors when desired torque was based on Time. An integral term (PID*) provided a small improvement in performance over $\mathrm{PD}^{*}$ control for Time and Angle based controllers. With Time-based high-level controllers, passivity (PAS) and previous-error compensation $\left(\mathrm{PD}^{*}+\mathrm{PEC}\right)$ provided a small benefit as well.

Table 4.5: Tracking errors with $\mathrm{PD}^{*}+\Delta \mathrm{LRN}$ torque control

\begin{tabular}{ccccccc}
\hline & & RMSE & $\% \tau_{\max }$ & & RMSE-A & $\% \tau_{\max }$ \\
\cline { 1 - 3 } \cline { 6 - 7 } Time & & $0.57 \pm 0.18 \mathrm{Nm}$ & $\mathbf{1 . 3 \%}$ & & $0.10 \mathrm{Nm}$ & $\mathbf{0 . 2 \%}$ \\
Angle & & $0.99 \pm 0.23 \mathrm{Nm}$ & $2.5 \%$ & & $0.11 \mathrm{Nm}$ & $0.3 \%$ \\
NMM & & $0.93 \pm 0.32 \mathrm{Nm}$ & $2.3 \%$ & & $0.12 \mathrm{Nm}$ & $0.3 \%$ \\
EMG & $2.14 \pm 0.77 \mathrm{Nm}$ & $5.9 \%$ & & $0.22 \mathrm{Nm}$ & $0.6 \%$ \\
\hline
\end{tabular}

\subsection{Discussion}

In this study, we investigated the effectiveness of several prominent torque control techniques, implemented on a tethered ankle exoskeleton, with unidirectional series-elastic actuation, during human walking, with a variety of high-level assistance controllers. We found that model-free proportional control with damping injection compensated 

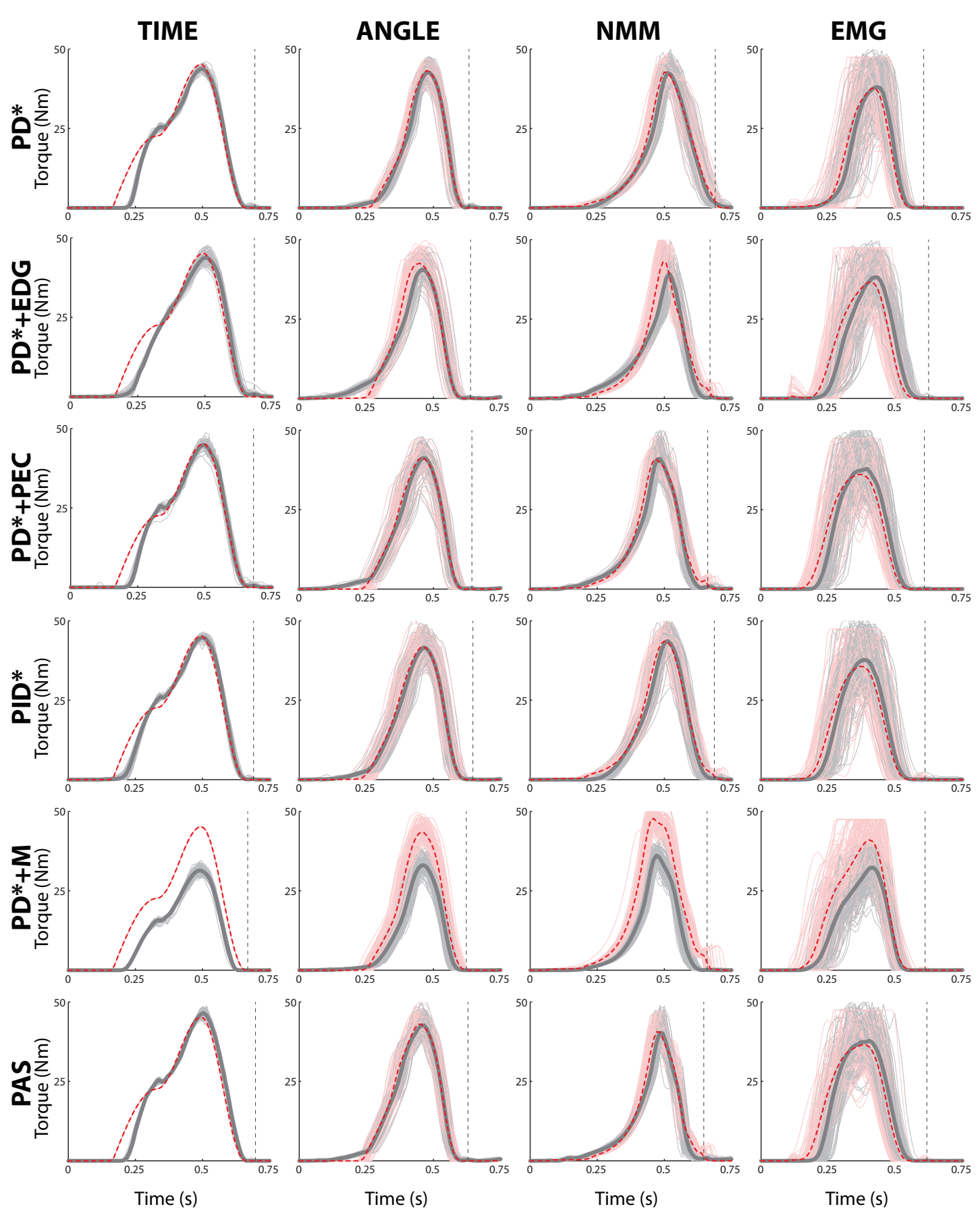

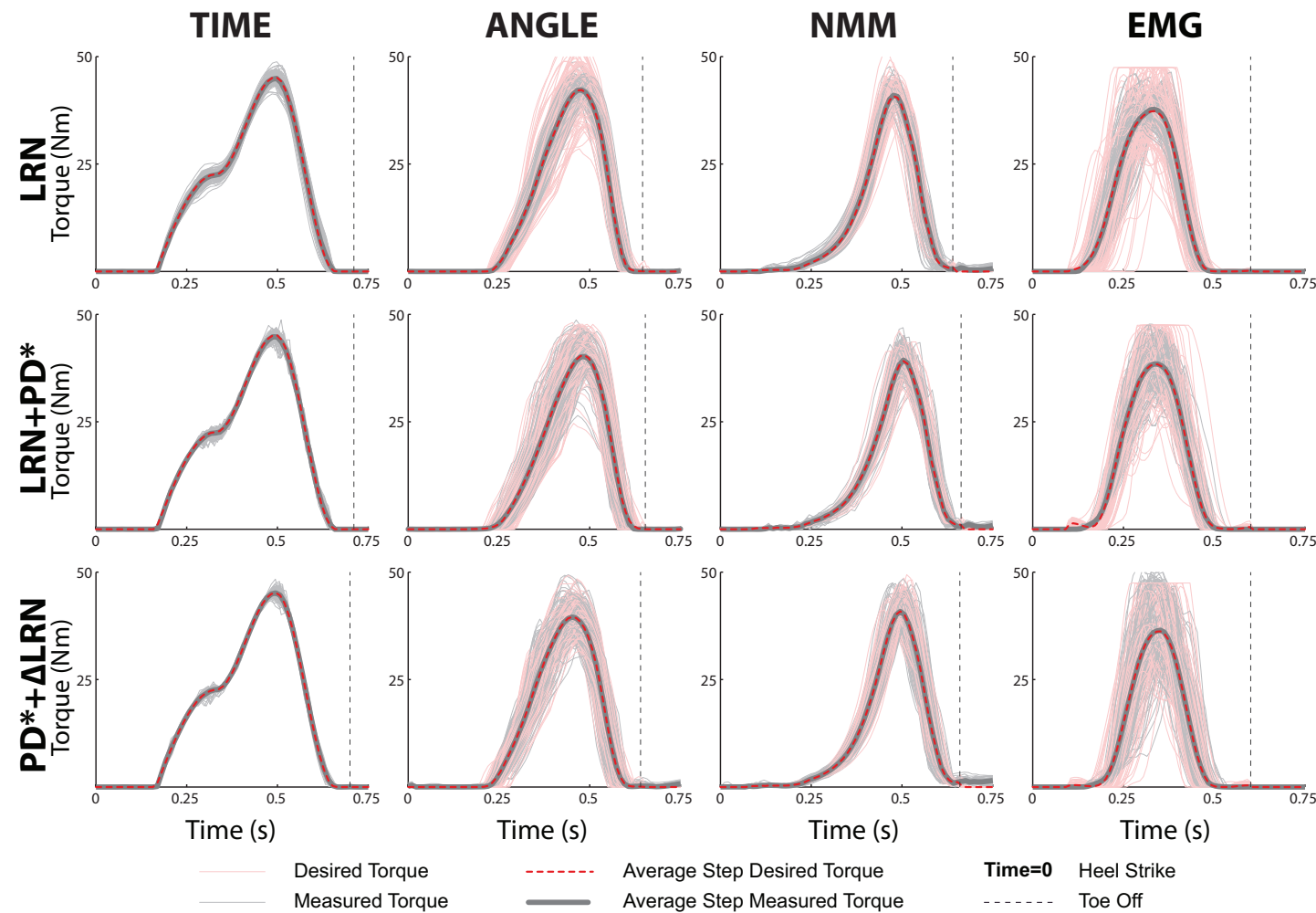

Figure 4.10: Time trajectories of desired torque (pink dots) and measured torque (gray dots) for 100 strides of walking, and average-stride desired torque (red line) and measured torque (gray line), for all combinations of controllers.

by iterative learning (Fig. 4.12) resulted in the lowest torque errors for all high-level controllers, both in real-time and for an averaged trajectory. This controller resulted in improved normalized torque tracking errors compared to prior exoskeleton torque control techniques.

\subsubsection{Proportional-learning-damping control}

The most successful controller identified in this study has features that are analogous to those of classical proportional-integral-derivative control: a proportional term provides tracking during transients, iterative learning eliminates steady-state cyclic errors, and damping injection provides stability. We might therefore label the approach 'proportional-learning-damping' control. 


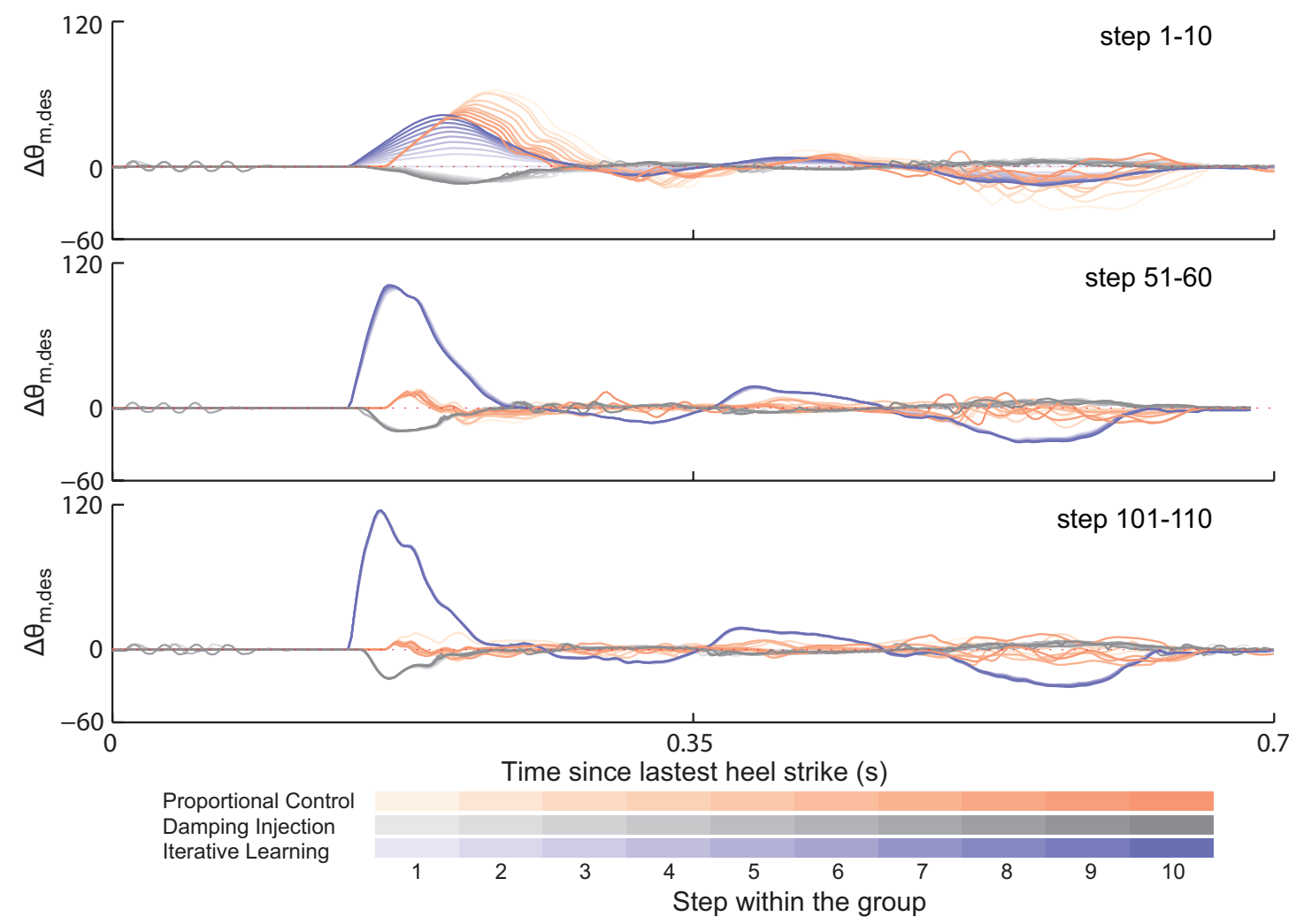

Figure 4.11: The contributions of each component of the PD* $+\Delta \mathrm{LRN}$ controller at steps 110 , steps 51-60 and steps 101-110. In the first step proportional control dominates, and at steady state the learned component dominates. Data shown are from the Time-based high-level controller. Plots for all high-level conditions are available as Supplementary Materials (Fig. S5).

Each component of the proportional-learning-damping controller contributes to overall commands in different ways across the learning process. During the first few walking steps, proportional control is the primary contributor, moderated by damping injection, while the learned trajectory remains near its initial value (Fig. 4.11, steps 1-10). At steady state, inputs are primarily the result of learned trajectories, which anticipate and override damping injection, while proportional control compensates for step-by-step variations in required input (Fig. 4.11, steps 91-100). This results in strong performance during transients and exceptional performance at steady state.

Designing and tuning the proportional-learning-damping controller is straightforward. First, the proportional gain on torque error is slowly increased until some overshoot and oscillations are observed. Next, the damping gain on motor velocity is in- 


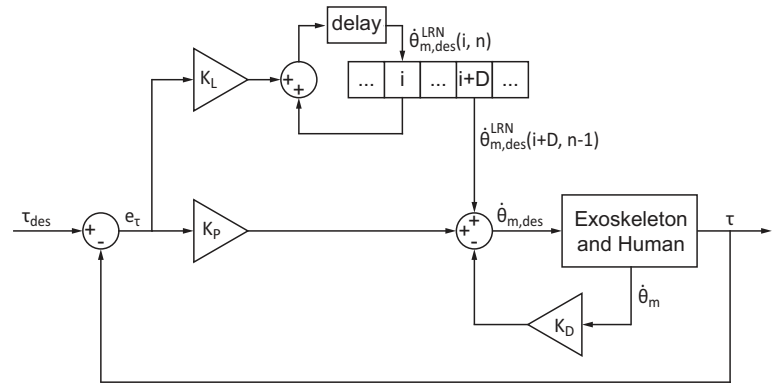

Figure 4.12: A block diagram of controller ${ }_{L} 9, \mathrm{PD}^{*}+\Delta \mathrm{LRN}$

creased until high-frequency motor oscillations are observed, and the gain is backed off of this limit. The proportional gain is then re-tuned such that it is as high as possible without resulting in oscillations in torque error. Next, the learning gain is set to a value of one tenth that of the proportional gain and fine-tuned until convergence occurs within the desired time, in this case ten strides. Finally, a parameter sweep is performed on the delay parameter used to preview learned desired motor position. The effects of learning are sensitive to this choice; without a value very close to optimal, ripples in the learned desired motor position will form and grow. In those cases, non-unity values of the forgetting and error filtering terms are required to stabilize the system. With the correct choice of delay, however, ripples did not form during at least one thousand strides in our experiments, even without forgetting or error filtering terms. We expect that a similar tuning process would be effective for a wide variety of lower-limb exoskeleton systems.

This approach builds on the strengths of torque control techniques implemented in prior lower-limb exoskeletons. Feedback control terms similar to those tested in this study have been used in Bowden-cable driven hip-knee exoskeletons [88], hip-knee exoskeletons with collocated drives [91] and mobile hip exoskeletons [82]. Effective joint position tracking has been achieved in a knee exoskeleton using an iterative learning approach analogous to that tested in this study [105]. Improvements in torque tracking have been achieved in a Bowden-cable driven hip-knee exoskeleton using a lower-dimensional 'kernel-based' version of the iterative learning approach tested in 
this study [92]. The proportional-learning-damping controller identified in this study incorporates the most effective permutations of these previously-identified control concepts for the present system.

Comparisons to prior torque tracking results are complicated by differences in hardware. For example, the present system has higher-power off-board motors than most exoskeletons, and the unidirectional Bowden cable can go slack during the swing phase, eliminating the need for active control to achieve transparency. Some prior exoskeletons have estimated joint torques using simplified system models rather than direct measurement, which can result in the appearance of low-error torque tracking despite substantial unmeasured torque errors. Nevertheless, the proportional-learning-damping controller identified in this study achieved the lowest torque errors as a percentage of desired torque of any exoskeleton to date.

It is likely that iterative learning, of the form used here or in other studies, would improve torque tracking during most human locomotor activities, since even irregular gaits exhibit some degree of repeatability. The case of the EMG-based high-level controller provides an insight into such scenarios, because the EMG signal contains substantial noise and is highly variable from step to step (Fig. 4.10; Fig. S2). Despite these irregularities, the addition of a learning component reduced torque tracking errors by $45 \%$ compared to feedback control alone in the EMG condition.

Further improvements in torque tracking for some high-level controllers might have been possible with alternate phase variables. Iterative learning resulted in the greatest improvements in torque tracking for the Time-based high-level controller, presumably because motor position adjustments were also learned in time. Learning as a function of ankle angle in the Angle condition, for example, might have resulted in greater improvements. However, time provides a unique and monotonically increasing phase variable, with consistent indexing across steps, capable of capturing control inputs with 
very high levels of complexity. This topic merits further exploration.

Since the completion of this study, this approach has been used on multiple devices and applications used in gait assistance [175] which demonstrated the generality of the method.

\subsubsection{Benefits of additional control elements}

There appeared to be some interactions between high-level and low-level control elements, which might provide insights into strategies for circumstances that were not tested in this experiment.

\section{Continuous-time integration:}

When proportional-damping control was augmented by an integral term (PID*), previouserror compensation $\left(\mathrm{PD}^{*}+\mathrm{PEC}\right)$, or passivity-based adaptation (PAS), torque error with Time-based high-level control was slightly improved. However, these continuous-time integral components showed no effect or negative effects in tracking performance for other high-level controllers. We can identify two factors that may explain the ineffectiveness of integral control in these cases. One factor is integral windup; the rapid changes in set point over the course of the stride could lead to either excessive or insufficient error accumulation. Another factor is the nonlinear, changing, delayed dynamics of the system; the Bowden cable has nonlinear stiffness, both the transmission and human change in time, and there are delays in the control loop and in motor actuation. All of these factors are known to limit integral control performance due to the linear accumulation of torque errors that are not linearly comparable [135]. In the case of the Time-based high-level controller, continuous-time integration may have been more effective due to the consistency of the desired torque in time, which may have translated 
into more constant torque errors than with other high-level controllers.

The apparent success of integral terms in other lower-limb exoskeletons with Bowdencable transmissions may be due to differences in torque sensing, cable composition or activities tested. For example, controllers of the Lopes system have typically included an integral term on torque error $[88,95,92]$. Differences might relate to torque sensing. In the present study, torque was measured at the joint using strain gauges. In Lopes, torque has been estimated from relative angle of the motor and joint or from series spring deflection, which might result in a more linear relationship between motor angle input and apparent joint torque. Differences might also relate to hardware. The Bowden cables in Lopes have features that could make them more consistent with a simple spring model, such as stiffer, pre-stretched steel cables and bidirectional drives that do not allow slack. Differences might also relate to the characteristic behavior of the joint being assisted. Lopes assists the hip and knee, which have relatively smooth, continuous patterns of joint torque. In this study, we assisted the ankle joint, which typically involves sharper changes in dynamics. For example, the foot intermittently contacts the ground, discontinuously changing both the impedance of the ankle joint and the magnitude of desired torques. These changes connote rapid changes in set point for the controller, which leads to windup of integral control elements, among other challenges.

\section{Model-based control elements:}

Although model-based control elements show promise in simulation and in theory, these generally worsened or had no effect on tracking performance in our experiment. One might expect that better performance could have been obtained with a more accurate estimate of model parameters in the $\mathrm{PD}^{*}+\mathrm{M}$ controller. However, in exploratory tests we found that the best performance was obtained by driving model-based contributions to zero. One reason for the ineffectiveness of model-based compensation may 
have been the nonlinear stiffness of the Bowden cable, which we modeled as a linear spring, and slow changes in cable stiffness and length due to heating over the course of each trial. Another reason may have been the exclusion of friction and stiction. However, in pilot tests using model-based compensation that included these elements, we found them to make the controller less robust; in each case the effects were highly sensitive to choice of parameter value and torque tracking error was not reduced. Sensitivity to model errors seems to be a fundamental issue in implementing this type of inverse-model control in exoskeletons with Bowden cable transmissions.

The passivity-based controller (PAS) fared slightly better, perhaps due to its adaptive nature, but still did not yield substantial benefits. One factor that may have limited its effectiveness is input mismatch; the controller was designed with motor voltage as input (Eq. 4.18), but implemented using motor velocity commands instead. While these terms are closely related (Eq 4.11), it is possible that an alternate mode of motor control, or an alternate formulation, could have led to improved results. Another factor that may have limited performance was the inclusion of a term akin to continuous-time integration, which is subject to windup as discussed above. The primary limitations likely stem from the reliance on any explicit model, however, since the dynamics of this human-exoskeleton system are highly complex and time-varying.

Some prior exoskeleton controllers have used model-based control elements, apparently to good effect. For example, [91] used continuous-time integration and modelbased terms that were not found to improve performance in the present study. This may be because the Bowden cable transmission used here relocated heavy actuators off of the leg, making gravity compensation unnecessary, but had more unmodeled dynamics than a gear train, making friction compensation less effective. 


\section{Gain scheduling, optimal control, and learning:}

Error-dependent gains $\left(\mathrm{PD}^{*}+\mathrm{EDG}\right)$ did not provide benefits for any high-level controller. Lower gains when torque errors were low seem to have led to larger errors at other times, since the set point changed rapidly and there were substantial execution delays. Gain scheduling methods that instead use optimal control might improve torque tracking for this system, but such feedback control techniques would still be limited by communication and actuation delays. By contrast, iterative learning realizes another form of optimal control, but uses a feed-forward approach to overcome delays. The iterative learning controller developed here is a variation on one-dimensional root finding using Newton's method. The problem is to find the desired motor position ( ${ }_{L}^{7}$ and $L^{8}$ ) or displacement $\left(L^{9}\right)$ for zero torque error, i.e., to solve the function:

$$
e_{\tau}\left(\Delta \theta_{m, d e s}\right)=0
$$

The solutions are approximated in an iterative manner by

$$
\Delta \theta_{m, d e s}(n+1)=\Delta \theta_{m, d e s}(n)-K_{l} \cdot e_{\tau}(n)
$$

which can be rewritten as

$$
\Delta \theta_{m, d e s}(n+1)=\Delta \theta_{m, d e s}(n)-\frac{e_{\tau}\left(\Delta \theta_{m, d e s}(n)\right)}{K_{l}^{-1}}
$$

This demonstrates a variation of Newton's method to solve $e_{\tau}=0$ with the estimate

for the derivative of $e_{\tau}$ fixed as $e_{\tau}^{\prime}=K_{l}^{-1}$. Unlike gain scheduling in feedback control, this optimal control approach addresses control delays through the combination of a 
feed-forward term and a delay-compensating prediction term. Therefore, even with an optimized gain schedule, $\mathrm{PD}^{*}+\mathrm{EDG}$ probably would not out-perform $\mathrm{PD}^{*}+\Delta \mathrm{LRN}$.

\subsubsection{Factors Limiting Interpretation}

\section{High-level controllers:}

It is difficult to make comparisons across high-level controllers for the same low-level torque controller, because tracking difficulty may vary with desired torque pattern. For example, we can imagine a Time-based trajectory with step changes in desired torque for which precise tracking would be infeasible. Similarly, the ease of tracking Anglebased desired torques likely depends on the similarities between the target torque-angle curve and the passive relationship arising from series elasticity. EMG-based desired torques generally seem to be difficult to track, given the unpredictable signals that directly drive desired torque, but increasing filter frequency could make this task easier. We did not test multiple values for high-level parameters in this experiment, which is an area for future work.

\section{Interactions with human response:}

We designed this experiment with the implicit expectation that low-level torque control would not significantly affect human response to high-level assistance modes, but this does not appear to have been the case. Changes in the patterns of desired torque (Fig. 4.10) and joint kinematics (Supporting Materials, Section II) across torque controllers within the same high-level controller reveal an interaction effect. For example, we found more variability in joint kinematics with $\mathrm{PD}^{*}$ torque control than with LRN torque control when desired torque was generated on the basis of Time. In this case, dif- 
ferences seem to be related to the smoothness of the measured torque generated by the two controllers; the subject reported that the PD* controller had uncomfortable oscillations, leading to compensatory activity, while the LRN controller did not. As another example, we found more variability in desired torque with NMM-based assistance than Angle-based assistance using $\mathrm{PD}^{*}$ torque control, but an opposite trend using LRN torque control. This appears to be the result of complex, multi-time-scale, dynamic interactions between continuous behavior of the torque controller, within-stride variations by the human, high-level control responses, and human adaptation over multiple strides. These effects may also be important in selecting and tuning an exoskeleton torque controller.

\section{Hardware dependence:}

Exoskeleton hardware, particularly series compliance, also interacted with the quality of torque tracking. We performed pilot tests with no series spring, other than the Bowden cable, and found significant increases in torque error and subject discomfort for all control combinations. We also tried more compliant series springs, and found small increases in torque tracking error. When series stiffness is too high, we expect small position changes by the human to result in large, undesirable, changes in torque, and when it is too low we expect motor dynamics to limit performance [31]. Interactions between series elasticity, low-level torque control, high-level assistance, and assisted task could be explored in the future.

Some of the control elements found to be ineffective in this system might be more effective in exoskeletons with different hardware or task characteristics. For example, there are many examples of exoskeleton controllers incorporating model-based terms, often accompanied by hardware that lends itself more readily to modeling [e.g. 91]. Experimental comparisons of torque tracking performance with and without model-based 
terms in these systems would lend further insights into their potential contributions to effective torque tracking in exoskeletons, and might provide a useful point of contrast to the present work. Future work could also address the effects of exoskeleton hardware changes as an additional dimension in the space sampled here. Measuring performance for each combination of a set of high-level controllers, low-level controllers and exoskeleton hardware setups would provide the best insights into interactions between these features. As the dynamical contributions of nonlinear time-varying elements, intermittent contact or forceful human interactions increase, continuous-time integral terms, gain scheduling, and model-based compensations are expected to become less effective, as observed with the unidirectional Bowden-cable-driven anklefoot exoskeleton used in this study. The present system therefore represents a more challenging case for torque control, suggesting that the proportional-learning-damping controller identified here would perform even better in a system with simpler, more consistent dynamics.

The unidirectional Bowden cable used in this study made torque control more challenging, but is not the dominant factor in the observed patterns of torque error. The onset of applied torque tended to lag that of desired torque, particularly with feedback controllers and TIME based desired torque. This pattern might suggest that slack in the Bowden cable as the primary cause of torque error, but this is not the case. All high-level controllers set desired torque to zero during the beginning of stance, from about 0 to $0.25 \mathrm{~s}$, consistent with typical human ankle torque patterns. All low-level controllers acted to track desired torque throughout stance, quickly eliminating slack from the previous swing phase early in the period of zero desired torque. The Bowden cable was therefore not slack upon the onset of desired torque. This is evident from the pattern of torque with feedback control and ANGLE based desired torque, which led desired torque beginning at about $0.10 \mathrm{~s}$. Patterns in torque error across controllers are better-explained by a combination of rapid changes in desired torque, rapid movements of the human, and electromechanical delays in applying desired changes in motor po- 
sition. These issues are common to most lower-limb exoskeletons, especially systems using series-elastic actuation.

\subsubsection{Implications for control of future systems}

The insights gained from this study are expected to help guide the design of torque controllers for systems with similar traits, particularly complex, changing dynamics and cyclic motions, such as lower-limb exoskeletons, active lower-limb prostheses and walking robots. Based on the present results, there is reason to expect that the combination of feedback control and feed-forward iterative learning, without continuous-time integration or model-based compensation, will provide strong torque tracking performance in any such system. Other control elements might further improve performance for some systems and control objectives. For example, if system dynamics are relatively constant and easy to identify, model-based compensation might be useful. If the measured and desired torque both change slowly, continuous-time integral control may also lead to some improvements. In any case, proportional-learning-damping control is expected to provide good baseline torque tracking.

\subsection{Conclusions}

We performed a systematic comparison of exoskeleton torque control techniques under realistic operating conditions, and found that proportional control with damping injection compensated by iterative learning resulted in smaller torque errors relative to peak torque than any other approach tested or previously demonstrated. Designing this proportional-learning-damping controller was straightforward, requiring sequential tuning of only four parameters. Our results generally support such an approach for any torque-controlled exoskeleton used during locomotion. The complex interactions be- 
tween exoskeleton hardware, torque control, assistance control, task goals and human behavior remain a rich area for future research.

\subsection{Supporting Materials}

\section{S1. Sequence of experimental conditions}

Experiments were conducted over the course of 3 days, with all data for each highlevel assistance controller collected on the same day. Low-level torque controllers were collected in the same order for each high-level controller, except for a change in the order of the PAS controller for the Time-based desired torque (which was accidentally skipped, then caught at the end of the collection). The exact sequence is shown in Table SI.

\begin{tabular}{|c|c|c|c|c|}
\hline \multirow{3}{*}{ Sequence } & \multirow{2}{*}{$\begin{array}{c}\text { Day } 1 \\
\text { Afternoon }\end{array}$} & \multicolumn{2}{|c|}{ Day 2} & \multirow{2}{*}{$\begin{array}{c}\text { Day } 3 \\
\text { Afternoon }\end{array}$} \\
\hline & & Morning & Afternoon & \\
\hline & TIME & NMM & ANGLE & EMG \\
\hline \multirow{4}{*}{$\downarrow$} & $\mathrm{PD}^{*}$ & $\mathrm{PD}^{*}$ & $\mathrm{PD}^{*}$ & $\mathrm{PD}^{*}$ \\
\hline & $\mathrm{PD}^{*}+\mathrm{M}$ & $\mathrm{PD}^{*}+\mathrm{M}$ & $\mathrm{PD}^{*}+\mathrm{M}$ & $\mathrm{PD}^{*}+\mathrm{M}$ \\
\hline & PID* $^{*}$ & PID* $^{*}$ & PID $^{*}$ & PID* \\
\hline & $\mathrm{PD}^{*}+\mathrm{EDG}$ & $\mathrm{PD}^{*}+\mathrm{EDG}$ & $\mathrm{PD}^{*}+\mathrm{EDG}$ & $\mathrm{PD}^{*}+\mathrm{EDG}$ \\
\hline \multirow[t]{3}{*}{$\downarrow$} & $\mathrm{PD}^{*}+\mathrm{PEC}$ & $\mathrm{PD}^{*}+\mathrm{PEC}$ & $\mathrm{PE}+\mathrm{PEC}$ & $\mathrm{PD}^{*}+\mathrm{PEC}$ \\
\hline & LRN & PAS & PAS & PAS \\
\hline & LRN+PD* & LRN & LRN & LRN \\
\hline \multirow[t]{2}{*}{$\downarrow$} & $\mathrm{PD}^{*}+\Delta \mathrm{LRN}$ & $\mathrm{LRN}^{+} \mathrm{PD}^{*}$ & $\mathrm{LRN}+\mathrm{PD}^{*}$ & $\mathrm{LRN}+\mathrm{PD}^{*}$ \\
\hline & PAS & PR+LRN & $\mathrm{PD}^{*}+\Delta \mathrm{LRN}$ & $\mathrm{PD}^{*}+\Delta \mathrm{LRN}$ \\
\hline
\end{tabular}

Table SI: Data collection timeline

\section{S2. Ankle Angle versus Time}

Fig. S1 shows trajectories of exoskeleton ankle joint angles in time for 100 strides, and a time-averaged trajectory, for each combination of high- and low-level controller. 
With Ankle-based and NMM-based desired torque, trajectories were similar to those of the biological ankle joint during normal human walking. With Time-based and EMGbased desired torque, less ankle excursion occurred on an average step. Variability in joint angle was highest when desired torque was based on Time.

\section{S3. Torque versus Ankle Angle}

Fig. S2 shows ankle torque versus ankle angle for 100 strides, and the time-averaged torque versus time-averaged joint angle, for each combination of high- and low-level controller. With Ankle-based and NMM-based desired torque, trajectories were similar to those of the biological ankle joint during normal human walking. With EMGbased desired torque, torque-angle curves have consistent shape across torque controllers, characterized by very steep slopes in angle space with approximately infinite impedance in places. With Time-based desired torque, torque-angle curves were complex and nonlinear. Changes in torque-angle relationships across low-level torque controllers within the same high-level controller (within the same column) suggest either interactions between torque control mode and human coordination pattern, or subject adaptations over time. Curves tend to move in a clockwise direction, indicating positive work production by the exoskeleton.

Fig. S3 shows the ankle torque versus ankle angle curve for 100 strides, and the timeaveraged torque versus time-averaged joint angle, for the case when motor position was held constant. This passive relationship between joint angle and joint torque seemed to be related to the difficulty of tracking desired torques generated by different high-level controllers; desired curves that were closer to the passive curve seemed easier for, e.g., PD* control, while LRN control made larger improvements when the two curves were dissimilar. 


\section{S4. T-Test Results for Controller RMS-E Values}

Table SII-SV shows the t-test mean comparison $p$ value of every two low level torque controllers within each high level controller. We used a significance level of $\alpha=0.05$. P-values that are beyond the threshold are shown in red. Almost all comparisons were highly statistically significant. Please see main text for effect size information.

\begin{tabular}{|c|c|c|c|c|c|c|c|c|}
\hline & $\mathrm{PD}^{*}$ & $\mathrm{PD}^{*}+\mathrm{EDG}$ & $\mathrm{PD}^{*}+\mathrm{PEC}$ & PID $^{*}$ & $\mathrm{PD}^{*}+\mathrm{M}$ & PAS & LRN & $\mathrm{LRN}+\mathrm{PD}^{*}$ \\
\hline $\mathrm{PD}^{*}+\mathrm{EDG}$ & $1.1 \mathrm{e}-22$ & . & . & . & . & . & . & . \\
\hline $\mathrm{PD}^{*}+\mathrm{PEC}$ & $1.4 \mathrm{e}-20$ & $4.2 \mathrm{e}-42$ & . & . & . & . & . & . \\
\hline PID $^{*}$ & $6.1 \mathrm{e}-34$ & $1.5 \mathrm{e}-52$ & $2.9 \mathrm{e}-08$ & . & . & . & . & . \\
\hline $\mathrm{PD}^{*}+\mathrm{M}$ & $2.1 \mathrm{e}-169$ & $4.2 \mathrm{e}-140$ & $1.2 \mathrm{e}-174$ & $4.0 \mathrm{e}-182$ & . & . & . & . \\
\hline PAS & $1.3 \mathrm{e}-06$ & $3.5 \mathrm{e}-32$ & $2.8 \mathrm{e}-05$ & $7.5 \mathrm{e}-17$ & $1.1 \mathrm{e}-175$ & . & . & . \\
\hline LRN & $1.9 \mathrm{e}-94$ & 7.1e-113 & $1.0 \mathrm{e}-84$ & $8.9 \mathrm{e}-81$ & $4.6 e-178$ & $6.1 \mathrm{e}-93$ & . & . \\
\hline LRN+PD* & $6.3 e-153$ & $2.1 \mathrm{e}-107$ & $2.7 \mathrm{e}-144$ & $5.2 \mathrm{e}-114$ & $7.9 \mathrm{e}-182$ & $7.5 \mathrm{e}-126$ & $3.7 \mathrm{e}-08$ & . \\
\hline $\mathrm{PD}^{*}+\Delta \mathrm{LRN}$ & $8.4 \mathrm{e}-157$ & $9.8 \mathrm{e}-110$ & 8.0e-149 & $2.7 e-118$ & $1.1 \mathrm{e}-182$ & $1.7 \mathrm{e}-129$ & $1.5 \mathrm{e}-15$ & $5.6 \mathrm{e}-10$ \\
\hline
\end{tabular}

Table SII: Time-based desired torque RMS-E t-test $p$ values.

\begin{tabular}{|c||c|c|c|c|c|c|c|c|}
\hline & PD $^{*}$ & PD*+EDG & PD*+PEC & PID* & PD*+M & PAS & LRN & LRN+PD* \\
\hline PD $^{*}+$ EDG & $4.3 \mathrm{e}-143$ & $\cdot$ & $\cdot$ & $\cdot$ & $\cdot$ & $\cdot$ & $\cdot$ & $\cdot$ \\
\hline PD $^{*}+$ PEC & $9.3 \mathrm{e}-01$ & $5.0 \mathrm{e}-145$ & $\cdot$ & $\cdot$ & $\cdot$ & $\cdot$ & $\cdot$ & $\cdot$ \\
\hline PID $^{*}$ & $1.3 \mathrm{e}-06$ & $7.2 \mathrm{e}-151$ & $6.1 \mathrm{e}-07$ & $\cdot$ & $\cdot$ & $\cdot$ & $\cdot$ & $\cdot$ \\
\hline PD $^{*}+\mathrm{M}$ & $3.4 \mathrm{e}-164$ & $6.7 \mathrm{e}-130$ & $2.8 \mathrm{e}-162$ & $6.1 \mathrm{e}-164$ & $\cdot$ & $\cdot$ & $\cdot$ & $\cdot$ \\
\hline PAS & $4.1 \mathrm{e}-01$ & $1.3 \mathrm{e}-129$ & $4.5 \mathrm{e}-01$ & $1.8 \mathrm{e}-07$ & $6.1 \mathrm{e}-173$ & $\cdot$ & $\cdot$ & $\cdot$ \\
\hline LRN & $8.3 \mathrm{e}-06$ & $7.5 \mathrm{e}-15$ & $8.8 \mathrm{e}-06$ & $1.2 \mathrm{e}-07$ & $2.6 \mathrm{e}-60$ & $1.8 \mathrm{e}-05$ & $\cdot$ & $\cdot$ \\
\hline LRN+PD* & $7.2 \mathrm{e}-58$ & $7.0 \mathrm{e}-130$ & $4.9 \mathrm{e}-58$ & $1.0 \mathrm{e}-48$ & $4.3 \mathrm{e}-195$ & $1.0 \mathrm{e}-58$ & $2.8 \mathrm{e}-19$ & $\cdot$ \\
\hline PD $^{*}+\Delta$ LRN & $1.5 \mathrm{e}-52$ & $4.1 \mathrm{e}-130$ & $8.4 \mathrm{e}-53$ & $1.1 \mathrm{e}-42$ & $1.4 \mathrm{e}-193$ & $4.1 \mathrm{e}-53$ & $1.1 \mathrm{e}-17$ & $2.1 \mathrm{e}-02$ \\
\hline
\end{tabular}

Table SIII: Angle-based desired torque RMS-E t-test $p$ values.

\begin{tabular}{|c||c|c|c|c|c|c|c|c|}
\hline & PD $^{*}$ & PD & \\
\hline PD $^{*}+$ EDG & $4.4 \mathrm{e}-113$ & $\cdot$ & $\cdot$ & $\cdot$ & $\cdot$ & $\cdot$ & $\cdot$ & $\cdot$ \\
\hline PD $^{*}+$ PEC & $3.0 \mathrm{e}-11$ & $1.1 \mathrm{e}-86$ & $\cdot$ & $\cdot$ & $\cdot$ & $\cdot$ & $\cdot$ & $\cdot$ \\
\hline PID $^{*}$ & $3.2 \mathrm{e}-08$ & $3.2 \mathrm{e}-104$ & $2.5 \mathrm{e}-02$ & $\cdot$ & $\cdot$ & $\cdot$ & $\cdot$ & $\cdot$ \\
\hline PD $^{*}+\mathrm{M}$ & $1.3 \mathrm{e}-238$ & $3.1 \mathrm{e}-175$ & $6.3 \mathrm{e}-172$ & $2.5 \mathrm{e}-213$ & $\cdot$ & $\cdot$ & $\cdot$ & $\cdot$ \\
\hline PAS & $1.2 \mathrm{e}-11$ & $9.3 \mathrm{e}-85$ & $8.1 \mathrm{e}-01$ & $1.4 \mathrm{e}-02$ & $8.3 \mathrm{e}-169$ & $\cdot$ & $\cdot$ & $\cdot$ \\
\hline LRN & $7.5 \mathrm{e}-38$ & $7.1 \mathrm{e}-91$ & $5.7 \mathrm{e}-49$ & $4.2 \mathrm{e}-45$ & $9.4 \mathrm{e}-131$ & $2.3 \mathrm{e}-49$ & $\cdot$ & $\cdot$ \\
\hline LRN+PD & $2.6 \mathrm{e}-119$ & $1.3 \mathrm{e}-166$ & $1.4 \mathrm{e}-104$ & $2.1 \mathrm{e}-121$ & $1.6 \mathrm{e}-241$ & $4.1 \mathrm{e}-103$ & $4.1 \mathrm{e}-19$ & $\cdot$ \\
\hline PD* $+\Delta$ LRN & $8.2 \mathrm{e}-72$ & $1.5 \mathrm{e}-133$ & $8.9 \mathrm{e}-84$ & $1.2 \mathrm{e}-81$ & $3.0 \mathrm{e}-174$ & $1.0 \mathrm{e}-83$ & $8.4 \mathrm{e}-07$ & $1.3 \mathrm{e}-09$ \\
\hline
\end{tabular}

Table SIV: NMM-based desired torque RMS-E t-test $p$ values. 


\begin{tabular}{|c||c|c|c|c|c|c|c|c|}
\hline & PD $^{*}$ & PD $^{*}+$ EDG & PD $^{*}+$ PEC & PID $^{*}$ & PD $^{*}+\mathrm{M}$ & PAS & LRN & LRN+PD \\
\hline PD $^{*}+$ EDG & $1.9 \mathrm{e}-41$ & $\cdot$ & $\cdot$ & $\cdot$ & $\cdot$ & $\cdot$ & $\cdot$ & $\cdot$ \\
\hline PD $^{*}+$ PEC & $9.9 \mathrm{e}-15$ & $4.5 \mathrm{e}-20$ & $\cdot$ & $\cdot$ & $\cdot$ & $\cdot$ & $\cdot$ & $\cdot$ \\
\hline PID $^{*}$ & $1.7 \mathrm{e}-01$ & $3.0 \mathrm{e}-41$ & $1.4 \mathrm{e}-12$ & $\cdot$ & $\cdot$ & $\cdot$ & $\cdot$ & $\cdot$ \\
\hline PD*+M & $6.9 \mathrm{e}-52$ & $1.4 \mathrm{e}-06$ & $1.2 \mathrm{e}-32$ & $2.2 \mathrm{e}-51$ & $\cdot$ & $\cdot$ & $\cdot$ & $\cdot$ \\
\hline PAS & $1.4 \mathrm{e}-10$ & $1.5 \mathrm{e}-26$ & $4.5 \mathrm{e}-02$ & $2.5 \mathrm{e}-08$ & $1.9 \mathrm{e}-38$ & $\cdot$ & $\cdot$ & $\cdot$ \\
\hline LRN & $3.1 \mathrm{e}-04$ & $3.8 \mathrm{e}-10$ & $2.5 \mathrm{e}-01$ & $3.6 \mathrm{e}-03$ & $4.0 \mathrm{e}-17$ & $9.1 \mathrm{e}-01$ & $\cdot$ & $\cdot$ \\
\hline LRN+PD* & $7.5 \mathrm{e}-37$ & $1.0 \mathrm{e}-87$ & $4.0 \mathrm{e}-67$ & $3.8 \mathrm{e}-45$ & $4.6 \mathrm{e}-91$ & $1.5 \mathrm{e}-63$ & $1.5 \mathrm{e}-26$ & $\cdot$ \\
\hline PD $^{*}+\Delta$ LRN & $1.9 \mathrm{e}-34$ & $2.7 \mathrm{e}-84$ & $2.5 \mathrm{e}-63$ & $4.8 \mathrm{e}-42$ & $5.2 \mathrm{e}-89$ & $1.5 \mathrm{e}-59$ & $7.8 \mathrm{e}-26$ & $6.8 \mathrm{e}-01$ \\
\hline
\end{tabular}

Table SV: EMG-based desired torque RMS-E t-test $p$ values.

\section{S5. Convergence Time for Controllers with Iterative Learn- ing Components}

Fig. S4 shows convergence of RMS-E and RMS-E AVG tracking errors over time for the three torque controllers that involved iterative learning. Each dot denotes the RMSE or RMS-E AVG for a 20-step period starting from the stride number on the $\mathrm{x}$-axis. Solid lines are the exponential fits to the error trends. Convergence was slowest and the reduction in error greatest when desired torque was based on Time. Improvements were more modest with Angle- and NMM-based desired torque. When desired torque was based on EMG, learning resulted in large initial improvements in torque error, but high errors remained at steady state.

\section{S6. Relative contributions of the desired motor displace- ments of various components of $\mathbf{P D}^{*}+\Delta \mathrm{LRN}$ for all four high level controllers}

Fig. S5 shows convergence of RMS-E and RMS-E AVG tracking errors over time for the three torque controllers that involved iterative learning. Each dot denotes the RMSE or RMS-E AVG for a 20-step period starting from the stride number on the x-axis. 
Solid lines are the exponential fits to the error trends. Convergence was slowest and the reduction in error greatest when desired torque was based on Time. Improvements were more modest with Angle- and NMM-based desired torque. When desired torque was based on EMG, learning resulted in large initial improvements in torque error, but high errors remained at steady state. 

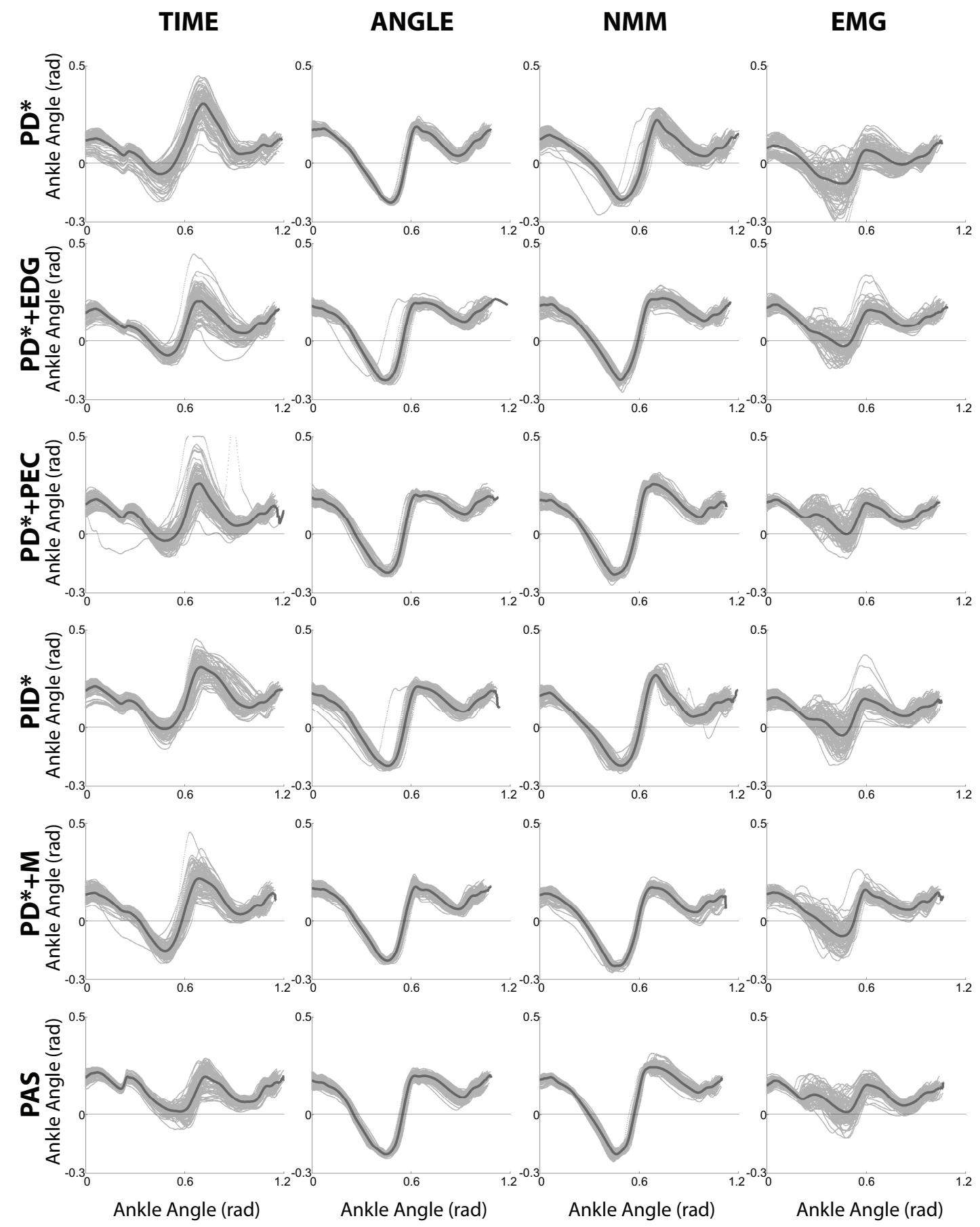

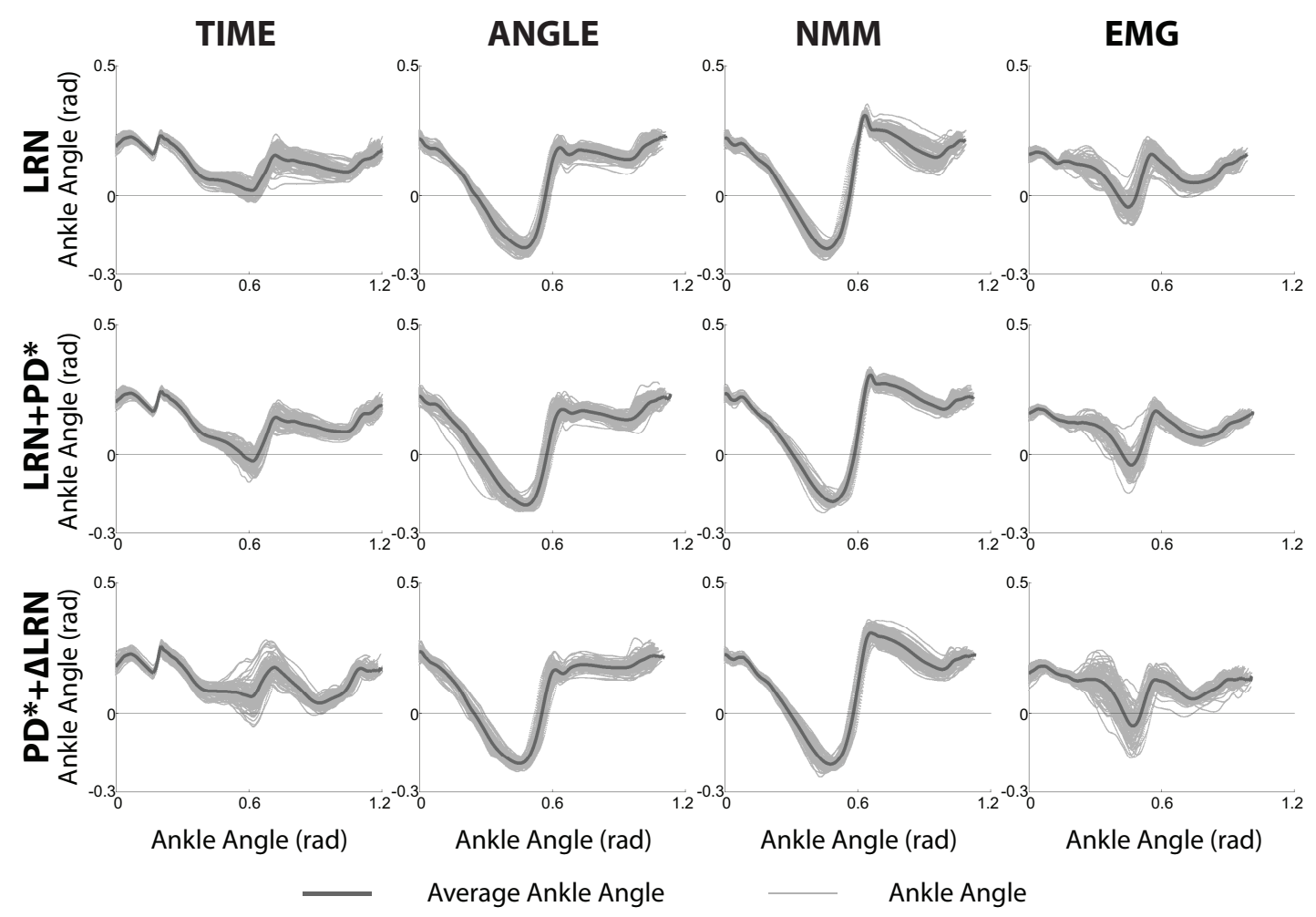

Fig. S1: Overlapped trajectories of measured ankle angle in time for 100 steady-state strides. 

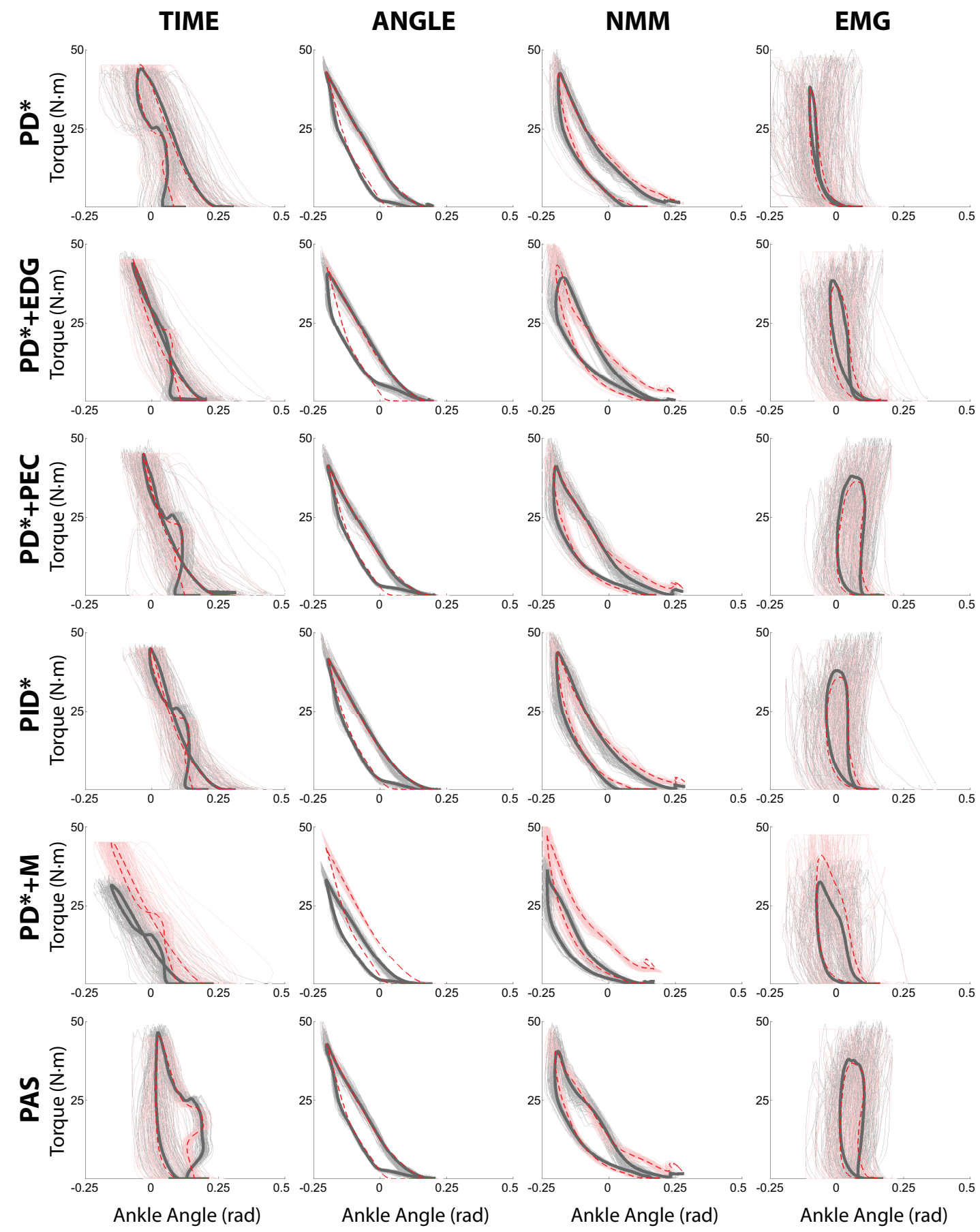

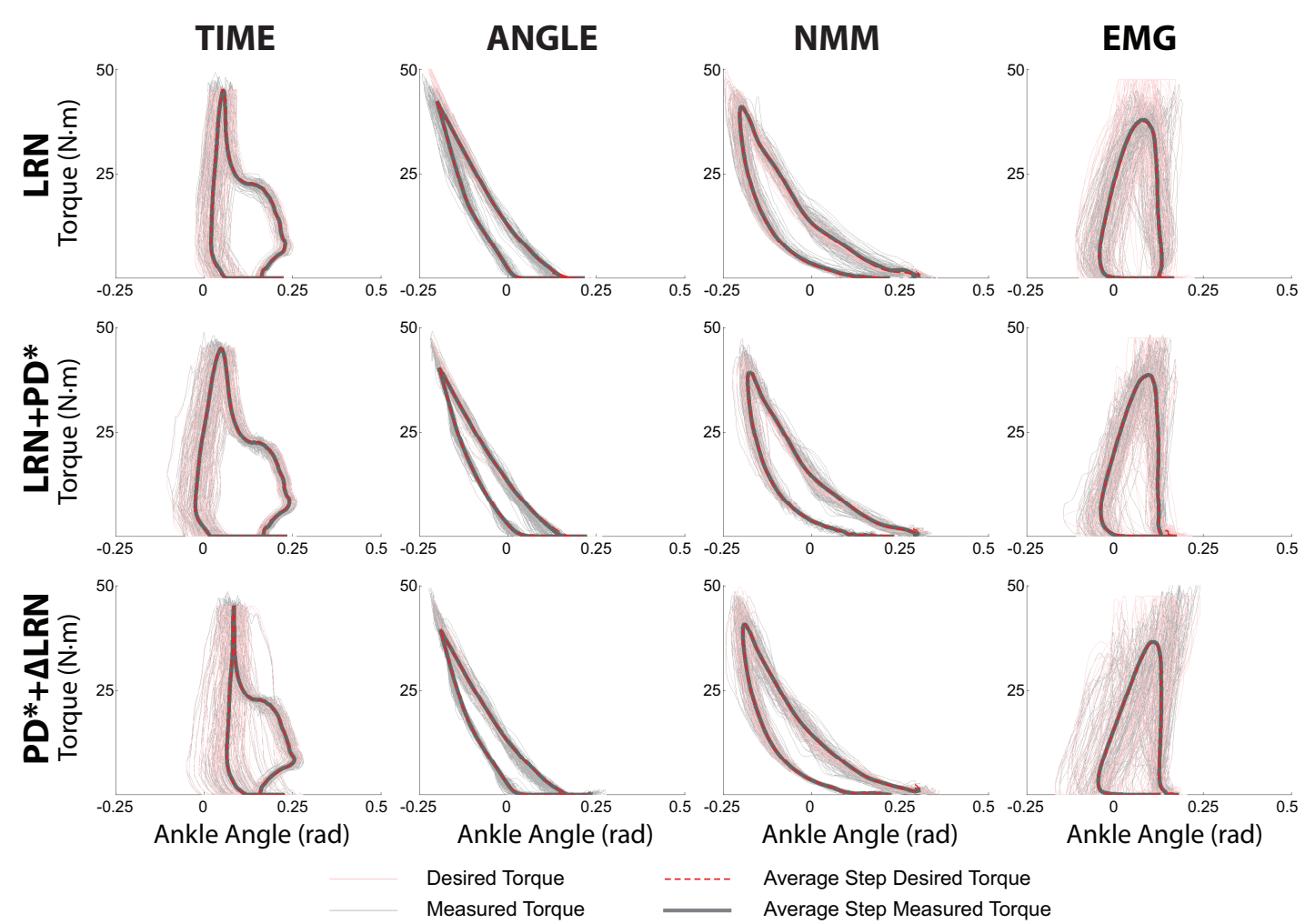

Fig. S2: Overlapped trajectories of measured ankle torque versus measured ankle angle for 100 steady-state strides.

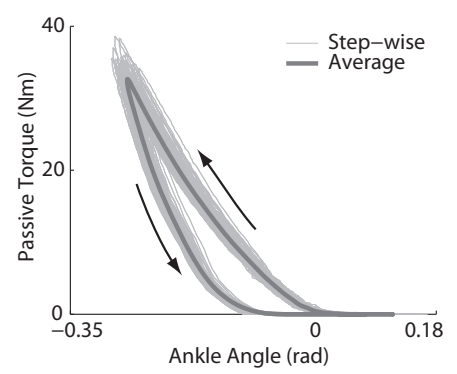

Fig. S3: Passive response: overlapped trajectories of measured ankle torque versus measured ankle angle for 100 strides during which the motor position was fixed.). 


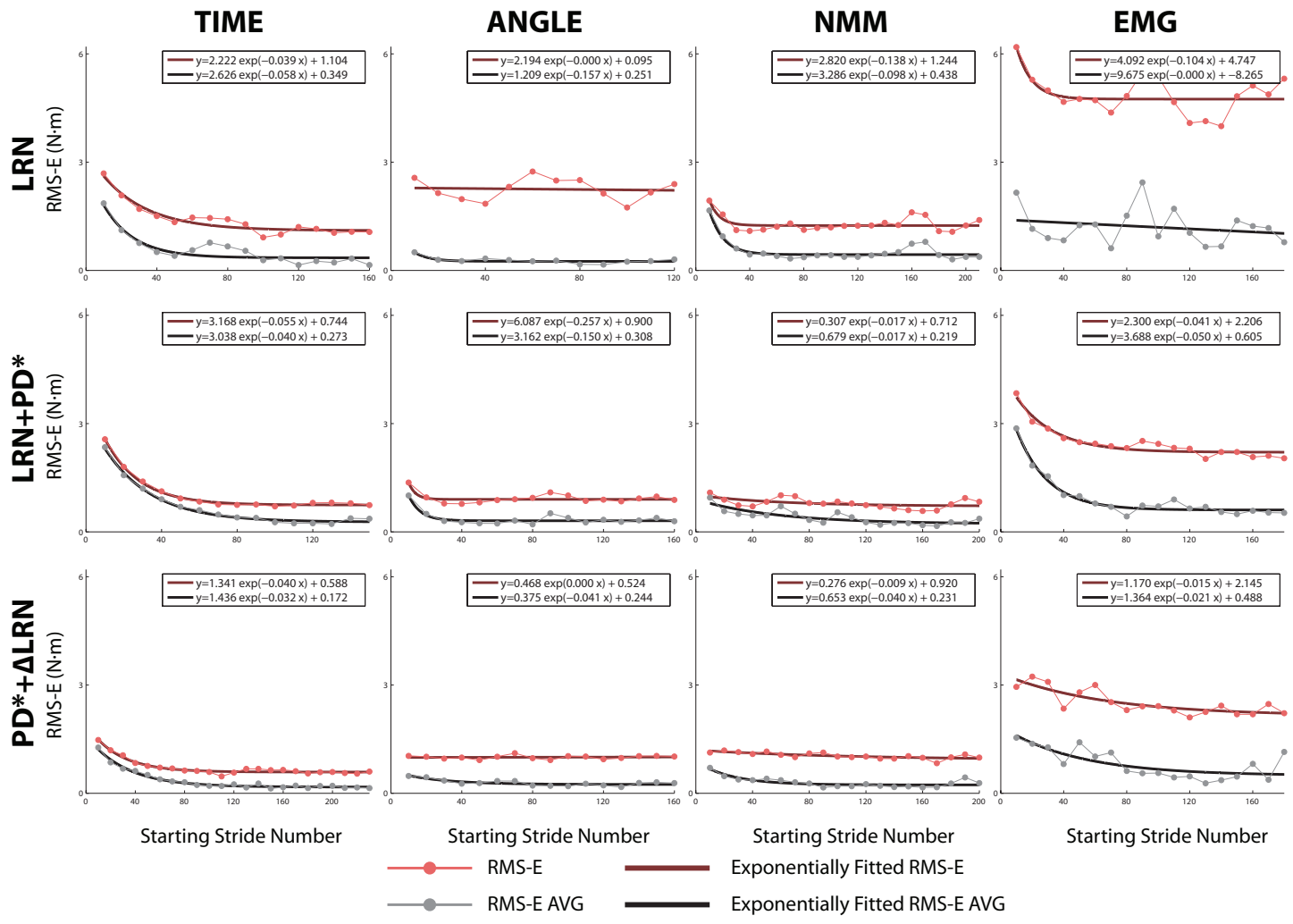

Fig. S4: Error as a function of time for controllers with iterative learning components. Each dot is the average RMS error for 20 strides, beginning with the number indicated on the $\mathrm{x}$ axis. 

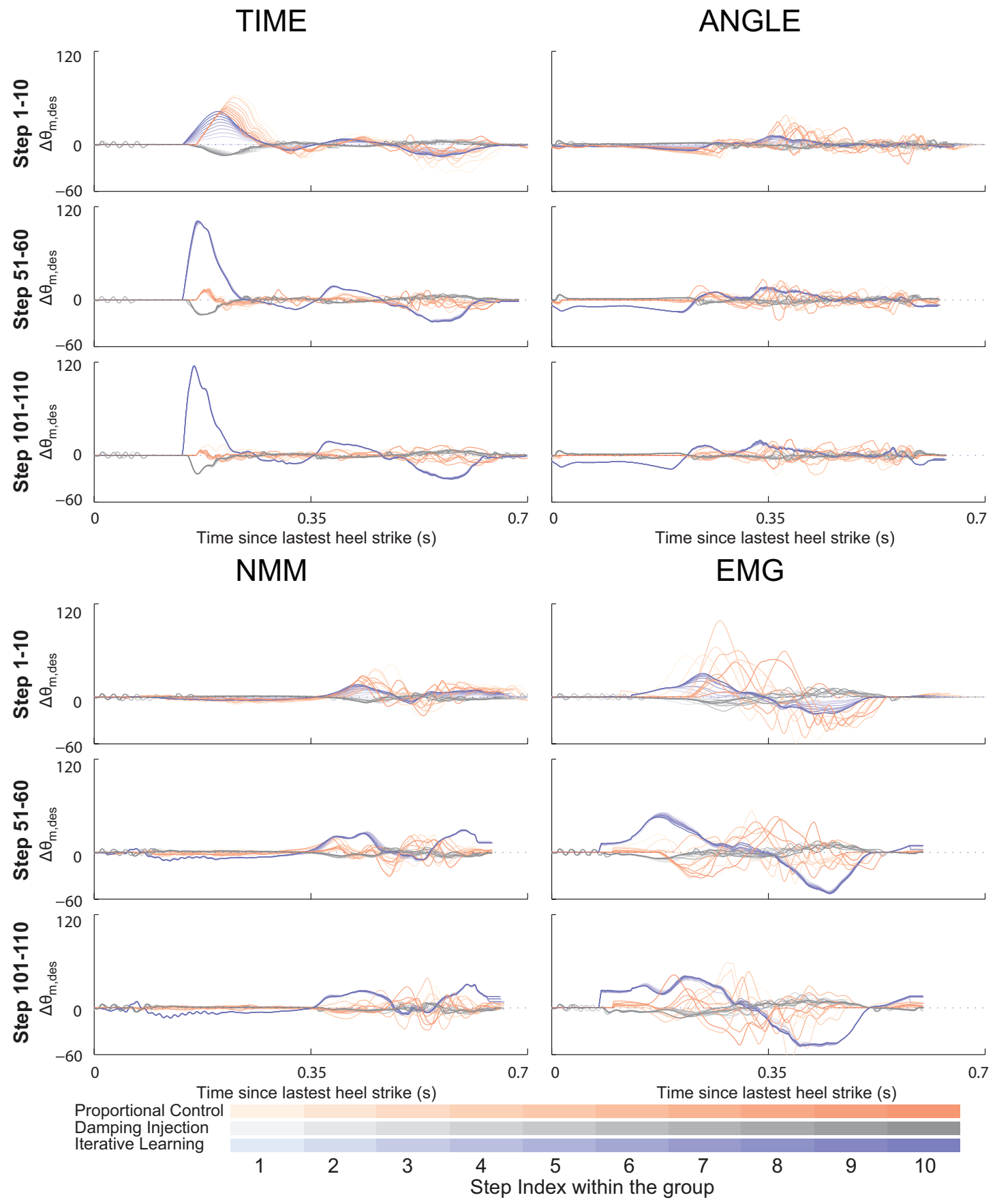

Fig. S5: Contributions from various elements towards the control input, the desired motor displacements, of $\mathrm{PD}^{*}+\Delta \mathrm{LRN}$ controller for four different high level controllers. 


\section{Chapter 5}

\section{Iterative Learning Gain Optimization}

\subsection{Introduction}

Direct manipulation of interaction forces or torques has been used in physical humanrobot interaction to reduce interface impedance, increase reactiveness of the robotic devices and thus improves human safety and comfort [26, 27]. Torque control also provides a simple means of manipulating the flow of energy from the exoskeleton to the human, which can be useful in biomechanics studies [28, 6, 29, 30, 172]. It can also be used to exploit passive dynamics or render virtual systems with alternate dynamics in humanoid robots [76], active prostheses [77-79], and exoskeletons [80-82, 7]. In exoskeletons and prostheses, quality of torque control is a limiting factor in precision of the applied intervention or assistance and can be the limiting factor in human-robot system performance. Therefore, there are always motives to improve torque control performance in these systems.

Various efforts have been made towards accurate torque tracking of lower-limb wearable robotic devices by means of controller design [92, 91, 60, 61]. This type 
of systems have complicated, changing and uncertain dynamics due to human-robot interactions and possible transmission frictions. Inter-subject and inter-step variations of human gait also introduce hard-to-quantify noise and disturbances. Such characteristics result in the effectiveness of a torque tracking structure made of the combination of model-free, integration-free feedback control and iterative learning [92, 60, 61]. In this control architecture, the iterative learning part ignores the complicated and changing system dynamics caused by human-robot interaction and transmission friction. It focuses on the transmission subsystem which was modeled as a linear system with relative degree of one. A P-type learning algorithm was used [37]. Iterative learning serves as a step-wise integral control and reduces steady-state errors by exploiting the cyclic behavior of walking. Due to its lagged response due to feed-forward characteristic and its dependence of effectiveness on the cyclicality of walking, iterative learning was expected to eliminate only errors nominal to stabilized gait. However, previous experiments show interactions of iterative learning gain with real-time tracking performance after stabilization of learning. This chapter explores the possibility to further improve real-time torque control performance in lower-limb exoskeletons, with limited knowledge of the complete system dynamics, by minimizing tracking errors causes by step-to-step gait variations using iterative learning gain of motor position command.

Since the proposal of the basic iterative learning control concept in the 1980s [136, 37], various techniques have been developed to optimize the learning gain. One approach was to enforce system convergence to follow some gradient of an objective function defined by the quadratic cost of tracking errors [137-139], or a weighted combination of tracking errors and change in control inputs [140]. Other works define learning gains by maximize convergence speed of control inputs [141, 142, 128, 143]. These early algorithms dealt with invariant and deterministic system dynamics. More recent work has discussed algorithms to compute optimal and sub-optimal iterative learning gains under measurement noises for time-varying linear [144] and nonlinear systems [145]. 
These previous works mainly optimized learning gains by expediting the convergence process of learning. In addition, fairly good knowledge of the system dynamics and noise level were known. However, in the problem of exoskeleton assisted-walking, there exist step-to-step gait variation and gait adaptation which makes walking not exactly periodical and results in tracking errors after stabilization of learning. The control architecture combining feedback and iterative learning depends mainly on the feedback part to contain these errors, which can be further improved by tuning the gain of the iterative learning part.

This study explores the possibility of optimize the post-stabilization real-time control performance of Arimoto's P-type learning control of motor position on lower-limb walking-related robots driven by series elastic actuators. Under the presence of complicated, varying and uncertain human-robot interaction and transmission friction dynamics, step-variance of human gait, this study investigates whether and how one can maximize real-time control performance by tuning the iterative learning gain with only a simplified partial model of the system and limited knowledge of gait variation distribution. Purpose of this study is to further improve torque tracking performance of control architecture in $[60,61]$ in lower-limb exoskeletons and other robotic locomotion systems.

\subsection{Methods}

The effects of iterative learning gain on the torque tracking performance of lower-limb exoskeletons are investigated on a tethered ankle-foot exoskeleton driven by a unidirectional Bowden cable $[7,60,61]$. Hypothesis for the optimal value iterative learning gain is made based on theoretical analysis and tested separately.

In testing the hypothesis, multiple desired quasi-stiffnesses, i.e., torque versus an- 

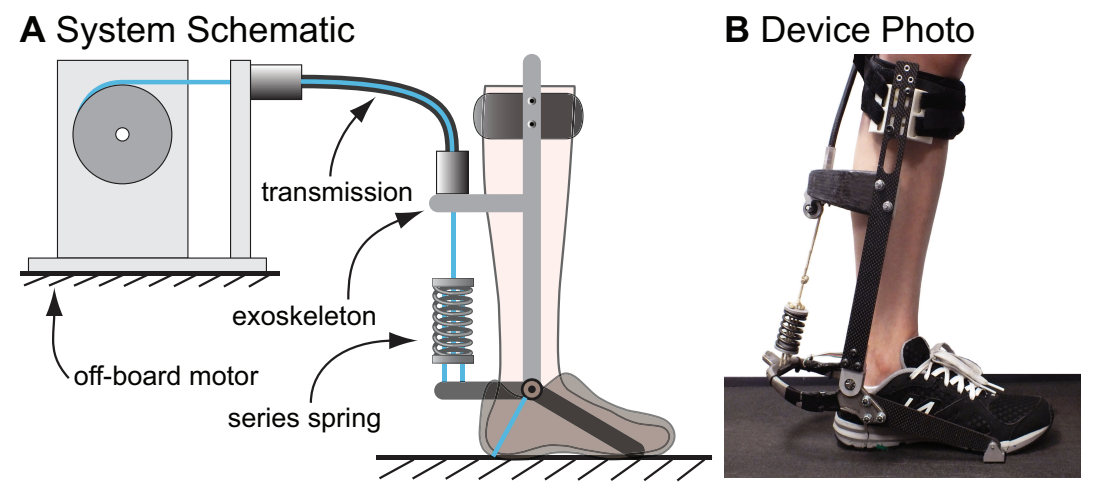

Figure 5.1: Tethered ankle exoskeleton system. (A) A schematic of the system that consists of an off-board motor system, a Bowden cable transmission ended with a series spring and an exoskeleton frame. For each desired quasi-stiffness, passive stiffness of the system was varied by switching the series spring and walking experiments were conducted to identify the optimal passive stiffness value. (B) A photo of the device worn by a subject standing on treadmill.

kle angle relationship were implemented, each tested with multiple actuator passive stiffnesses. For each of the desired stiffness and passive stiffness combination, multiple iterative learning gains were tested. Every experiment session denoted by a unique combination of learning Gain, desired stiffness and passive stiffness requires the subject to walk on the treadmill with a fixed speed for at least one hundred strides after stabilization of the learning process. The existence and value of the learning gain are then investigated by compare the torque tracking errors of different experiment sessions.

\subsubsection{Simplified System Model}

The system we investigated was a tethered ankle exoskeleton comprised of an off-board real-time control module and geared electric motor, a uni-directional Bowden cable transmission with a series spring, and an exoskeleton frame that interfaced with the human foot and shank (Fig. 5.1).

The following assumptions were made in generating the simplified model used for theoretical analysis of the system: 
$\diamond$ There is no friction in Bowden cable transmission, which means the tension in the cable are the same at the motor output pulley and the ankle exoskeleton sides at any time. We define this tension as $F$.

$\diamond$ The transmission system consisting of the Bowden cable and the series spring behaves like a linear spring, i.e.,

$$
F=K_{c} \cdot\left(r_{p} \cdot \theta_{p}-r_{a} \cdot \theta_{e}\right)
$$

in which $K_{C}$ is the total effective stiffness of the Bowden cable transmission and series spring; $\theta_{p}$ and $\theta_{e}$ are the pulley and exoskeleton joint angles relative to a position at which the Bowden cable begins to go slack; $r_{p}$ and $r_{a}$ are the pulley radius and the lever arm at the ankle joint.

$\diamond$ The joint movement of the exoskeleton is minor and the lever arm of the cable tension relative to the device joint is almost constant, i.e., $r_{a}$ is fixed and the torque at the exoskeleton side of the transmission is

$$
\tau=F \cdot r_{a}
$$

Denoting the aspect ratio of transmission as

$$
R=\frac{r_{p}}{r_{a}}
$$

and combining it with Eqs. (5.1) and (5.2), we can express the torque applied by the 
exoskeleton to the human ankle Therefore, the measured torque is

$$
\begin{aligned}
\tau & =F \cdot r_{a} \\
& =r_{a}^{2} \cdot K_{c}\left[\theta_{p} \frac{r_{p}}{r_{a}}-\theta_{e}\right] \\
& =K_{t}\left(\theta_{p} R-\theta_{e}\right)
\end{aligned}
$$

Transmission stiffness $K_{t}$ which relates torque at the exoskeleton to the angles of the motor output pulley and exoskeleton joint is defined as

$$
K_{t}=r_{a}^{2} \cdot K_{c}
$$

\subsubsection{Controllers}

\section{Low Level Control}

Previous work identified model-free, integral-action-free feedback control compensated by iterative learning as the most effective controller for lower-lime exoskeletons torque tracking during walking $[60,61]$. The final torque tracking performance of Eq. (5.6) is a combined effect of feedback control and iterative learning. In order to simplify the process, in analyzing and testing the existence of the optimal learning gains only iterative learning was used as the lower level controller for torque tracking. The format of the controller is described as:

$$
\begin{aligned}
\theta_{p, \text { des }}(i, n+1) & =\theta_{p, \text { des }}(i, n)-K_{l} \cdot e_{\tau}(i, n) \\
\dot{\theta}_{p, \text { des }}(i, n) & =\frac{1}{T}\left(\theta_{p, \text { des }}(i, n)-\theta_{p}\right) \\
\dot{\theta}_{m, \text { des }}(i, n) & =\frac{N}{T}\left(\theta_{p, \text { des }}(i, n)-\theta_{p}\right)
\end{aligned}
$$

where $i$ is the time index or number of control cycles elapsed within this stride, $n$ is this stride and $n+1$ is the next stride. This controller is divided into two parts: 
proportional control and damping injection as feedback, and iterative learning as feedforward. In this formulation, $e_{\tau}=\tau-\tau_{d e s}$ is torque error, $\tau$ is measured exoskeleton torque, $\tau_{d e s}$ is desired exoskeleton torque. Damping injection is used instead of derivative control to reduce the effect of measurement noise. $K_{l}$ is a iterative learning gain. The motor runs in velocity mode with the desire motor output pulley velocity $\dot{\theta}_{p, \text { des }}$ converted to desired motor velocity $\dot{\theta}_{m, \text { des }}$ before asserted. $T$ is a constant related to the rise time of motor position tracking and $N$ is the gear ratio of the motor.

\section{High Level Control: Linear Desired Quasi-Stiffness}

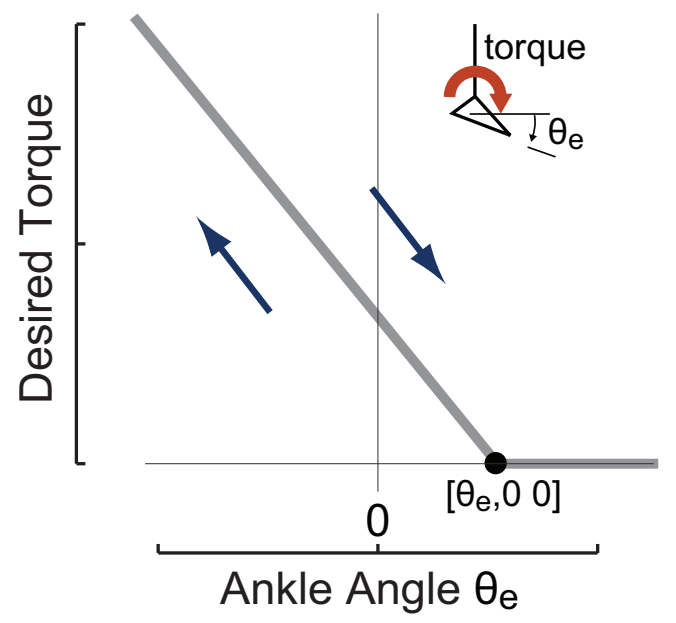

Figure 5.2: The ankle angle based high-level desired torque curve imposed in experiments to realize different desired quasi-stiffness profiles. It commands desired torque that is linearly proportional to exoskeleton joint angle $\theta_{e}$ defined by anchor point $\left[\theta_{e, 0} 0\right]$ and desired quasistiffness $K_{\text {des }}$.

The main high-level controller used in this study was a linear torque versus ankle angle curve, i.e., an equilibrium-controlled stiffness as shown in Fig. 5.2 and expressed in Eq. (5.7).

$$
\begin{aligned}
\tau_{d e s} & =-K_{d e s}\left(\theta_{e}-\theta_{e, 0}\right) \\
\tau_{d e s} & =\max \left(\tau_{d e s}, 0\right)
\end{aligned}
$$

where $\theta_{e, 0}$ denotes the maximum ankle position to apply external force and $K_{d e s}$ is a desired stiffness to be realized. 


\subsubsection{Theoretical Analysis on Optimal Iterative Learning Gain}

In this analysis, we assume perfect motor position tracking, i.e.,

$$
\theta_{p}(i, n) \equiv \theta_{p, d e s}(i, n)
$$

for any index $i . \theta_{p}$ is the measured motor pulley position and $\theta_{p, d e s}$ is the desired one. $n$ denotes the $n^{\text {th }}$ step and $i$ denotes the current index counted from the latest step start time. The iterative learning of desired motor position is expressed as [60, 61]

$$
\theta_{p, d e s}(i, n+1)=\theta_{p, d e s}(i, n)-K_{l} \cdot e_{\tau}(i, n)
$$

Force transmission is modeled in Eq. (4.6) and desired torque is set as Eq. (6.2)

In this study, we are interested in the real-time torque tracking performance under gait variation after stabilization, i.e., disturbance rejection performance of the controller. Therefore, we assume that at step $n-1$ and time index $i$, the learning controller has reached stabilization with perfect torque tracking, i.e.,

$$
\begin{aligned}
\tau(i, n-1) & =\tau_{d e s}(i, n-1) \\
e \tau(i, n-1) & =\tau(i, n-1)-\tau_{d e s}(i, n-1)=0
\end{aligned}
$$

The dynamic changes of the desired and generated torque in the next steps due to human gait changes are then investigated below.

Assuming an ankle kinematics change from step $n-1$ to $n$ at index $i$ by

$$
\Delta \theta_{e}(i, n)=\theta_{e}(i, n)-\theta_{e}(i, n-1)
$$


the desired torque changes by

$$
\begin{aligned}
\Delta \tau_{\text {des }}(i, n) & =\tau_{\text {des }}(i, n)-\tau_{\text {des }}(i, n-1) \\
& =-K_{d e s} \cdot\left[\theta_{e}(i, n)-\theta_{e}(i, n-1)\right] \\
& =-K_{d e s} \cdot \Delta \theta_{e}(i, n)
\end{aligned}
$$

Due to the assumption of perfect motor position tracking in Eq. (5.8), current desired/actual motor position is

$$
\begin{aligned}
\theta_{p, \text { des }}(i, n) & =\theta_{p, \text { des }}(i, n-1)-K_{l} \cdot e_{\tau}(i, n-1) \\
& =\theta_{p, \text { des }}(i, n-1)-K_{l} \cdot 0 \\
& =\theta_{p, \text { des }}(i, n-1)
\end{aligned}
$$

Therefore, combining Eq. (5.12) with Eq. (5.4), the measured torque at stride $n$ and index $i$ is

$$
\tau(i, n)=\tau(i, n-1)-K_{t} \cdot \Delta \theta_{e}(i, n)
$$

and the desired torque is

$$
\begin{aligned}
\tau_{d e s}(i, n) & =\tau_{d e s}(i, n-1)+\Delta \tau_{d e s}(i, n) \\
& =\tau_{d e s}(i, n-1)-K_{d e s} \cdot \Delta \theta_{e}(i, n)
\end{aligned}
$$

Combining equations (5.13) and (5.14), for step $n$ and index $i$, the torque error is

$$
\begin{aligned}
& e_{\tau}(i, n) \\
& =\tau(i, n)-\tau_{d e s}(i, n) \\
& =\tau(i, n-1)-K_{t} \Delta \theta_{e}(i, n)-\left[\tau_{d}(i, n-1)-K_{d e s} \Delta \theta_{e}(i, n)\right] \\
& =e_{\tau}(i, n-1)+\left(K_{d e s}-K_{t}\right) \Delta \theta_{e}(i, n) \\
& =0+\left(K_{d e s}-K_{t}\right) \Delta \theta_{e}(i, n) \\
& =\left(K_{d e s}-K_{t}\right) \Delta \theta_{e}(i, n)
\end{aligned}
$$

This means with perfect torque tracking in step $n-1$, the torque error of step $n$ is 
minimized when the desired and passive stiffness match. Next, at index $i$ of step $n+1$, the desired/actual motor position is

$$
\begin{aligned}
\theta_{p, d e s}(i, n+1) & =\theta_{p, d e s}(i, n)-K_{l} \cdot e \tau(i, n) \\
& =\theta_{p, d e s}(i, n)-K_{l} \cdot\left(K_{d e s}-K_{t}\right) \cdot \Delta \theta_{a}(i, n)
\end{aligned}
$$

In case of no changes in ankle kinematics from step $n$ to $n+1$ at time index $i$, i.e., $\theta_{e}(i, n+1)=\theta_{e}(i, n)$, then, $\tau_{d e s}(i, n+1)=\tau_{d e s}(i, n)$. The actual torque at current step and index is

$$
\begin{aligned}
& \tau(i, n+1) \\
& =K_{t}\left[\theta_{p}(i, n+1) R-\theta_{e}(i, n+1)\right] \\
& =K_{t}\left[\left(\theta_{p, d e s}(i, n)-K_{l}\left(K_{d e s}-K_{t}\right) \Delta \theta_{e}(i, n)\right) R-\theta_{e}(i, n+1)\right] \\
& =K_{t}\left[\left(\theta_{p, d e s}(i, n)-K_{l}\left(K_{d e s}-K_{t}\right) \Delta \theta_{e}(i, n)\right) R-\theta_{e}(i, n)\right] \\
& =K_{t}\left[\theta_{p, d e s}(i, n) R-K_{l}\left(K_{d e s}-K_{t}\right) R \Delta \theta_{e}(i, n)-\theta_{e}(i, n)\right] \\
& =\tau(i, n)-K_{t} K_{l}\left(K_{d e s}-K_{t}\right) R \Delta \theta_{e}(i, n) .
\end{aligned}
$$

Therefore, the latest torque error is

$$
\begin{aligned}
& e_{\tau}(i, n+1) \\
& =\tau(i, n+1)-\tau_{d e s}(i, n+1) \\
& =\tau(i, n+1)-\tau_{d e s}(i, n) \\
& =\tau(i, n)-K_{t} K_{l}\left(K_{d e s}-K_{t}\right) R \Delta \theta_{e}(i, n)-\tau_{d e s}(i, n) \\
& =\left(K_{d e s}-K_{t}\right) \Delta \theta_{e}(i, n)-K_{t} K_{l}\left(K_{d e s}-K_{t}\right) R \Delta \theta_{e}(i, n) \\
& =\left[\left(K_{d e s}-K_{t}\right)-K_{t} K_{l}\left(K_{d e s}-K_{t}\right) R\right] \Delta \theta_{e}(i, n)
\end{aligned}
$$

if $K_{t} \cdot K_{l} \cdot R=1$, i.e., $K_{l}=\frac{1}{K_{t} R}$, we have

$$
e_{\tau}(i, n+1)=0
$$

and balance is restored. 
However, if there is ankle kinematics change from step $n$ to $n+1$ at index $i$, i.e.,

$$
\Delta \theta_{e}(i, n+1)=\theta_{e}(i, n+1)-\theta_{e}(i, n) \neq 0,
$$

we have

$$
\tau_{d e s}(i, n+1)=\tau_{d e s}(i, n)-K_{d e s} \cdot \Delta \theta_{e}(i, n+1),
$$

and the actual torque at $(i, n+1)$ is

$$
\begin{aligned}
& \tau(i, n+1) \\
& =K_{t}\left[\theta_{p}(i, n+1) R-\theta_{e}(i, n+1)\right] \\
& =\tau(i, n)-K_{t} K_{l}\left(K_{d e s}-K_{t}\right) R \Delta \theta_{e}(i, n)-K_{t} \Delta \theta_{e}(i, n+1) .
\end{aligned}
$$

Combining equations (5.20) and (5.21), the torque error at $(i, n+1)$ is

$$
\begin{aligned}
e_{\tau}(i, n+1) \\
=\tau(i, n+1)-\tau_{d e s}(i, n+1) \\
=\left[\left(K_{d e s}-K_{t}\right)-K_{t} K_{l}\left(K_{d e s}-K_{t}\right) R\right] \Delta \theta_{e}(i, n) \\
\quad+\left(K_{d e s}-K_{t}\right) \Delta \theta_{a}(i, n+1)
\end{aligned}
$$

Assuming that the ankle kinematics change is bounded, i.e.,

$$
\left\|\Delta \theta_{e}(i, n)\right\| \leq \varepsilon
$$

the error at $(i, n)$ is

$$
\begin{aligned}
\left\|e_{\tau}(i, n)\right\| & =\left\|\left(K_{\text {des }}-K_{t}\right) \cdot \Delta \theta_{a}(i, n)\right\| \\
& \leq\left\|\left(K_{\text {des }}-K_{t}\right)\right\| \cdot\left\|\Delta \theta_{a}(i, n)\right\| \\
& =\left\|\left(K_{\text {des }}-K_{t}\right)\right\| \cdot \varepsilon
\end{aligned}
$$


The error at $(i, n+1)$ is then

$$
\begin{aligned}
& e_{\tau}(i, n+1) \\
&=\left[\left(K_{d e s}-K_{t}\right)-K_{t} \cdot K_{l} \cdot\left(K_{d e s}-K_{t}\right) \cdot R\right] \cdot \Delta \theta_{e}(i, n) \\
&+\left(K_{d e s}-K_{t}\right) \cdot \Delta \theta_{e}(i, n+1) \\
& \leq\left\|\left(K_{d e s}-K_{t}\right)-K_{t} \cdot K_{l} \cdot\left(K_{d e s}-K_{t}\right) \cdot R\right\| \cdot\left\|\Delta \theta_{e}(i, n)\right\| \\
&+\left\|K_{d e s}-K_{t}\right\| \cdot\left\|\Delta \theta_{e}(i, n+1)\right\| \\
& \leq {\left[\left\|\left(K_{d e s}-K_{t}\right)-K_{t} \cdot K_{l} \cdot\left(K_{d}-K_{t}\right) \cdot R\right\|+\left\|K_{d e s}-K_{t}\right\|\right] \cdot \varepsilon } \\
&= {\left[\left\|\left(K_{d e s}-K_{t}\right)\left(1-K_{t} \cdot K_{l} \cdot R\right)\right\|+\left\|K_{d}-K_{t}\right\|\right] \cdot \varepsilon } \\
& \leq {\left[\left\|K_{d e s}-K_{t}\right\| \cdot\left\|1-K_{t} \cdot K_{l} \cdot R\right\|+\left\|K_{d e s}-K_{t}\right\|\right] \cdot \varepsilon } \\
&=\left\|K_{d e s}-K_{t}\right\| \cdot\left[\left\|1-K_{t} \cdot K_{l} \cdot R\right\|+1\right] \cdot \varepsilon
\end{aligned}
$$

It is still in our best interest to assert

$$
1-K_{t} \cdot K_{l} \cdot R=0
$$

Therefore, we make the following hypothesis.

Hypothesis 1. There is an optimal iterative learning gain that benefits real-time torque tracking performance of iterative learning in exoskeleton assisted walking under a spring-like desired torque profile:

$$
K_{l, o p t}=\frac{1}{K_{t} R}
$$

\subsubsection{Testbed System Configurations}

We tested the hypothesis on a tethered ankle exoskeleton comprised of an off-board real-time control module and geared electric motor, a uni-directional Bowden cable transmission with a series spring, and an exoskeleton frame that interfaced with the 
human foot and shank (Fig. 5.1).

In the tethered ankle exoskeleton testbed as shown in Fig. 5.1, a dedicated real-time control system (ACE1103, dSPACE Inc.) sample sensors at $5000 \mathrm{~Hz}$, filter sensor data at $200 \mathrm{~Hz}$, and generate control commands at $500 \mathrm{~Hz}$. The motor unit was composed of a low-inertia 1.6 kW AC servo motor and a 5:1 planetary gear, with input voltage regulated by a motor driver running in velocity control mode (BSM90N-175AD, GBSM90MRP120-5 and MFE460A010B, Baldor Electric Co.). A digital optical encoder (E5, US Digital Corp.) measured motor position.

The exoskeleton frame applied forces on the front of the human shank below the knee, beneath the heel, and beneath the toe, so as to generate an ankle plantarflexion torque in proportion to transmission force. Torque was measured using strain gauges (MMF003129, Micro-Measurements) applied in a full Wheatstone bridge on the heel lever, with $1000 \mathrm{~Hz}$ signal conditioning (CSG110, Futek Inc.). Joint angle was measured using a digital optical encoder (E5, US Digital Corp.).

A flexible uni-directional Bowden cable transmitted forces from the motor to the exoskeleton frame while minimally restricting leg motions. The cable was composed of a coiled-steel outer conduit (415310-00, Lexco Cable Mfg.) and a $0.003 \mathrm{~m}$ diameter Vectran ${ }^{\circledR}$ inner rope, and was $2 \mathrm{~m}$ in length. A series spring was attached at the end of the rope to provide increased compliance. This spring is switched in tests to investigate the effects of changing passive stiffness on torque tracking in the device.

\subsubsection{Experimental Methods}

Since the purpose of experiments in this study was to quantify not the human reaction but the torque tracking performance of various control conditions, only one healthy subject ( $\mathrm{N}=1$, female, 32 years, $1.65 \mathrm{~m}, 56 \mathrm{~kg}$ ) was involved. The subject walked on a 
Table 5.1: List of desired stiffness tested in experiments with assigned ID

\begin{tabular}{c|ccc} 
Desired Stiffness ID & D1 & D2 & D3 \\
\hline $\mathbf{K}_{\mathbf{d e s}}(\mathbf{N m} / \mathbf{d e g})$ & 2 & 5 & 8.5
\end{tabular}

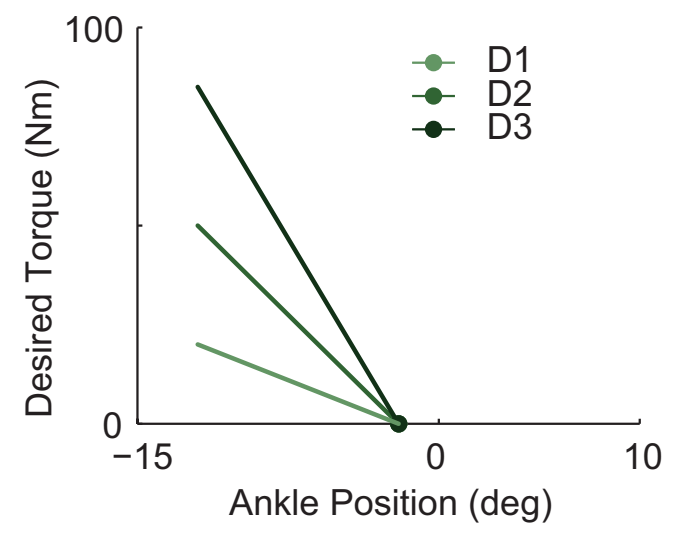

Figure 5.3: All three tested linear desired torque versus ankle angle curves used in the form of Eq. (6.2) with $\theta_{e, 0}=-2(\mathrm{deg})$ and $K_{d e s}$ values listed in Table 5.1.

treadmill with a fixed speed of $1.25 \mathrm{~m} / \mathrm{s}$ with a self-paced step frequency while wearing the tethered ankle exoskeleton on the right leg in all experiments. All experimental protocols were approved by Carnegie Mellon University IRB.

For the ease of readability, this chapter uses Newton-meter, degree and meter as the corresponding units for measurements of torque, angle and distance.

\section{Desired Torque Curves}

Three different desired quasi-stiffnesses in the form of Eq. (5.7) were implemented to test the hypothesis. In all cases, $\theta_{e, 0}=-2(\mathrm{deg})$, in which $\theta_{e}=0$ is defined by neutral standing position. The tested desired stiffness $K_{d e s}$ spans Table 5.1 with a maximum value that is 4.25 times the minimum. The resulted desired torque-ankleangle relationships are demonstrated in Fig. 5.3. 
Table 5.2: List of springs used in experiments with assigned ID

\begin{tabular}{c|ccc}
\hline Passive Stiffness ID & S1 & S2 & S3 \\
\hline Spring Part No. & DWC-148M-13 & DWC-187M-12 & No Spring \\
Length (m) & 0.0635 & 0.0508 & - \\
$\begin{array}{c}\text { Spring Rate } \\
\left(\mathbf{N} / \mathbf{m} \times 10^{3}\right)\end{array}$ & 15.1 & 50.1 & - \\
Max Load $(\mathbf{N})$ & 413.7 & 778.4 & - \\
\hline
\end{tabular}

\section{Realization of Different Passive Stiffness and Evaluation of Their Values}

For each of the desired stiffness profile defined by a torque-angle relationship, three passive series stiffness values of the transmission system were realized by changing the series spring of the ankle exoskeleton (Fig. 5.1.A). Two of them were achieved by attaching different compression springs(Diamond Wire Spring, Glenshaw, PA) at the end of the series elastic actuators. One was realized by getting rid of the spring in the structure, in which case the system passive stiffness is solely determined by the stiffness of the synthetic rope in Bowden cable. The list of springs used and their corresponding properties are available in Table 5.2.

The effective passive stiffness values of various spring configurations, $K_{t}$, are evaluated based on passive walking experiment data. For each of three passive stiffness configurations, the human subject walks on the treadmill for at least one hundred steady steps wearing the exoskeleton with the motor position fixed at the position where force starts to be generated with the subject standing in neutral position. Such walking sessions were repeated multiple times for the same passive stiffness along the study. For each session of one hundred steps, the instantaneous value of passive stiffness at each time stamp was calculated and presented in relation to the measured torque values. Fig. 5.4 presents such plots of passive walking sessions for different spring configurations, one session for each configuration. Median of the instantaneous passive stiffness values within the stabilized region was defined as the stabilized passive stiffness value of the session. For any spring configuration, its stabilized region is defined as a $5.65 \mathrm{Nm}$ 
torque range, within which the change of trend for the instantaneous passive stiffness averaged over all sessions is minimum. The mean of the stabilized passive stiffness values across multiple experimental sessions for the same passive stiffness configuration was then used as its effective passive stiffness value.

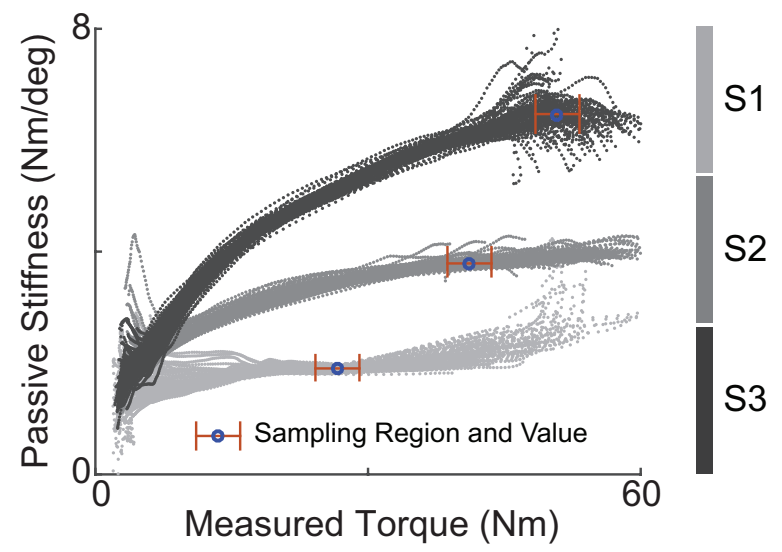

Figure 5.4: Instantaneous passive stiffness values of passive walking sessions are plotted against the measured torques for various spring configurations, one session for each. One experiment session consists of one-hundred steps with motor position fixed. The stablized passive stiffness value for one session is defined as the median of the values over a stabilized region. The effective passive stiffness of the one stiffness configuration is defined as the mean of stabilized stiffness values across multiple sessions.

\section{Experimental Procedures}

For each desired stiffness and passive stiffness combination, ten iterative learning gains that span a range with a 20-times difference between the maximum and minimum. Therefore, $3 \times 3 \times 10$ experiment sessions were conducted in total. During each experiment session defined by a unique combination of learning gain, desired stiffness and passive stiffness, the subject walked for at least one hundred steps after stabilization of the learning processes.

Four different definitions of torque errors were calculated for each combination as its performance indicators. The first as we call an 'absolute error' is defined as the mean of step-wise root-mean-squared torque errors over the one hundred stable steps. The second one is a 'relative error' that was defined by the absolute error divided by the mean 

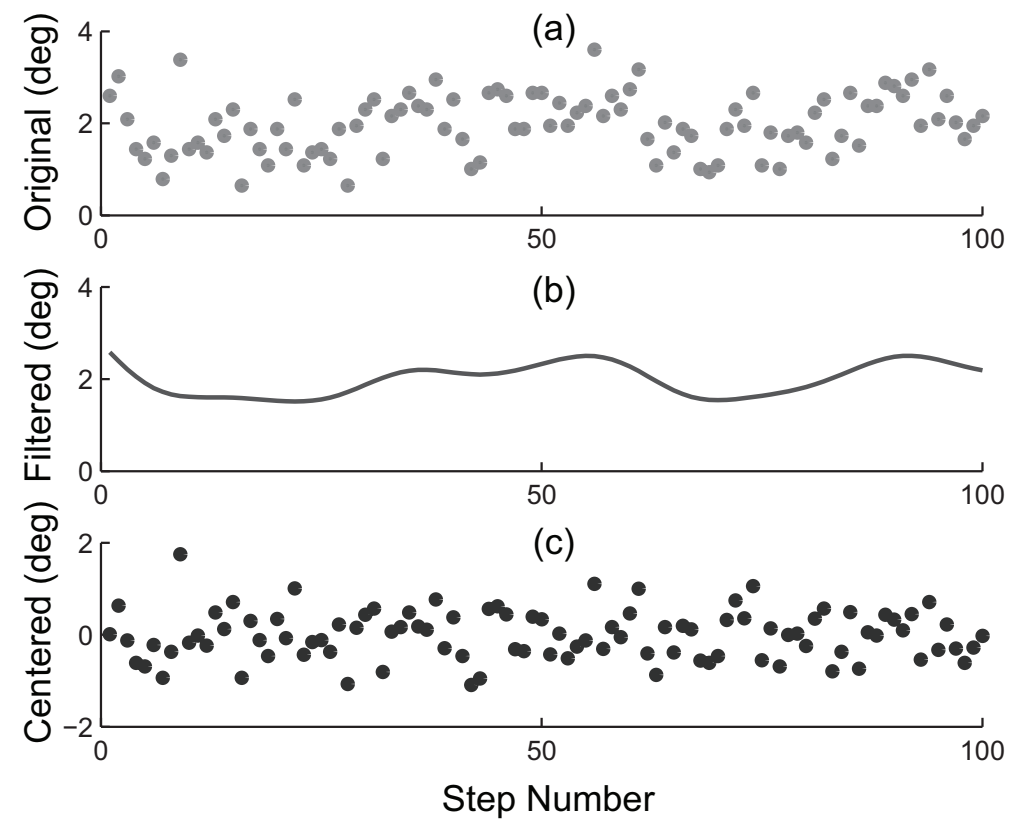

Figure 5.5: Centering process of index-wise ankle positions. a): Ankle position array of the one hundred steps investigated for an example time stamp within steps. b): Ankle position array as shown in a) zero-phase filtered with a 1/20 cut-off frequency butter-worth filter. c): Centered ankle position array achieved by subtracting array in b) from that in a).

of peak desired torque of the one hundred stable steps. A 'normalized absolute error' is also calculated in a similar way compared to the 'absolute error' except that all the instant torque error values were divided by the variance of centered ankle position array of the same time within a step over one hundred steps before used for the calculation of step-wise root-mean-squared values. The centered ankle position array is defined as the difference between the original array and filtered array as demonstrated in Fig. 5.5. The 'normalized relative error' is then calculated by dividing the 'normalized absolute error' by the mean peak desired torque.

For each desired and passive stiffness combination, each of the defined error indexes were plotted against their corresponding logarithm of their learning gains divided by the optimal gains to test the hypothesis. 
Table 5.3: List of passive stiffness values

\begin{tabular}{c|ccc} 
Desired Stiffness ID & $\mathbf{S 1}$ & $\mathbf{S 2}$ & $\mathbf{S 3}$ \\
\hline $\mathbf{K}_{\mathbf{T}}(\mathbf{N m} / \mathbf{d e g})$ & 1.9 & 3.7 & 5.9
\end{tabular}

\subsection{Results}

Their actual values of passive stiffness are calculated as the mean stabilized values across multiple data sets and are listed in Table 5.3. This passive stiffness set span a range with a 3.5 times difference between the maximum and minimum values.

The mean of root-mean-squared torque errors of various learning gains are presented in Fig. 5.6.

From the results, the torque errors normalized to the standard deviation of centered ankle position trajectories seem to be more aligned with the hypothesis, which states that the lowest real-time torque errors happens around an gain value $K_{l}=K_{l, o p t}=$ $1 / K_{t} R$. However, the slope between the error and learning gain is pretty shallow in the area around the expected optimal gain for all combinations of desired and passive stiffnesses. For each of the combination of passive stiffness and desired torque curve, the values of $\ln \left(\frac{K_{l}}{K_{l, o p t}}\right)$ and normalized absolute errors are fitted into a second order polynomial, and its minimum was used to identify the relative iterative learning, $\frac{K_{l}}{K_{l, o p t}}$ at which the error is minimized. The optimal $\frac{K_{l}}{K_{l, o p t}}$ values of all nine combinations are then calculated. Results showed that the optimal gain that minimized root-meansquared tracking errors after stabilization of learning process for all combinations is $0.9929 \pm 0.3846$ times the predicted optimum.

A $100 \%$ increase in learning gain only results in a $4.54 \%$ increase in normalized torque error on average. 
A. Mean of root-mean-squared errors
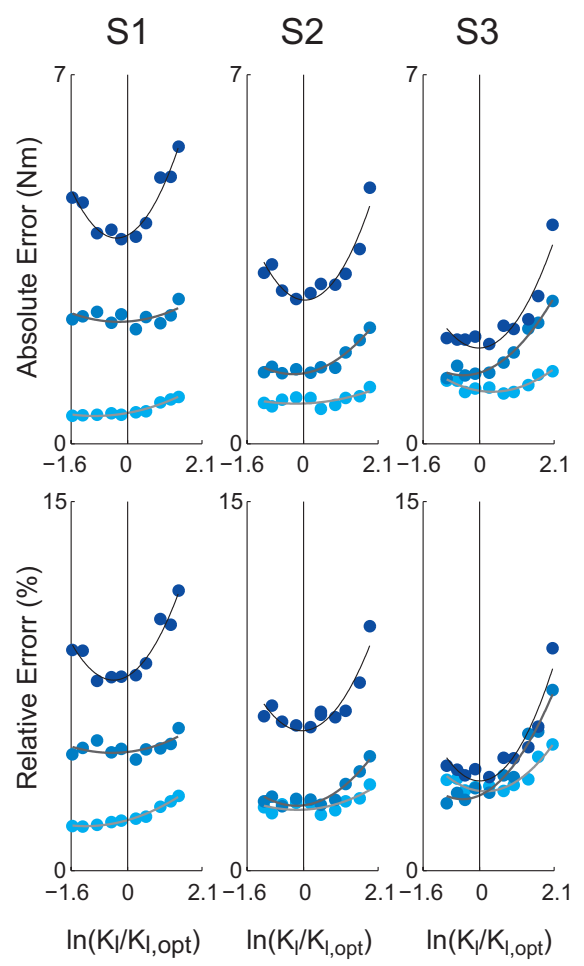

B. Mean of root-mean-square of errors normalized by variance of position-wise ankle position
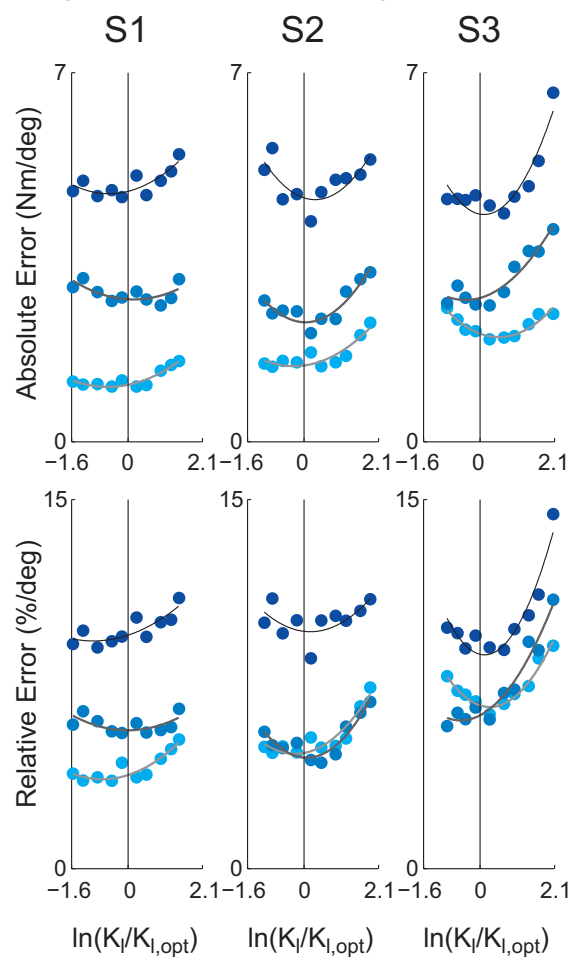

D1

D2

D3

Figure 5.6: Mean of step-wise root-mean-squared torque tracking errors of all combinations of learning gain, desired stiffness and passive stiffness and the relative errors to their peak desired torques are plotted against the natural logarithms of the learning relative to the predicted optimum $\ln \left(K_{l} / K_{l, o p t}\right.$. A: Values computed with raw torque errors. B: Values computed by normalizing raw torque errors by position-wise ankle position variance. 


\subsection{Discussion}

This study examines the possibility of optimizing the real-time torque tracking performance of iterative learning control after the stabilization of learning process in series elastic actuator driven walking robots by theoretical analysis and human walking experiments while wearing an active ankle exoskeleton. Theoretic analysis suggests that to optimize real-time torque tracking using iterative learning feed-forward control, the learning gain should be set as the inverse of the actuator passive stiffness with respect to the motor side. Among the various torque error indices investigated for walking experiments, torque errors normalized by the variance of the centered ankle position array demonstrate strongest agreement with the hypothesis. The optimal iterative learning identified through experiment is $0.9929 \pm 0.3846$ times the predicted optimal gain. These results show that this optimal learning gain mostly suppresses the torque errors due to the step-to-step variation of human gait after stabilization, but not those due to slow adaptation of human gaits. The results also suggest a shallow slope of tracking error increase when the learning deviates from the hypothesized optimum.

Due to the presence of nonlinear, uncertain, highly complex and changing dynamics and the employment of a highly simplified model, a lot of system features were not captured in the theoretical hypothesis, which led to imperfection in the alignment between theory and experiment results. One complication that contributed was the nonlinear property of the system passive stiffness due to the slow stretching property of the Vectran ${ }^{\circledR}$ cable as demonstrated by Fig. 6.3. Due to the unstructured changes of passive stiffness between different loads and trials, only one stabilized value was used for each passive stiffness configuration. Another feature that causes complication into system dyanmics but was not accounted for in theoretical analysis was the highly nonlinear, complex and changing frictions in Bowden cable. We also made the assumption of perfect motor position tracking, which is not true in practical cases due to the 
limitation of motor velocity.

Regardless of the imperfection of system modeling, the torque tracking errors did arrive at a minimum at the neighborhood of the hypothesized optimal iterative learning gain. The shallow slope of changes around the optimum value also suggests a rather relaxed learning gain tuning process. When the iterative learning gain spans a range of [50\% 200\%] relative to the theoretical optimum, the average increase in torque error is less than $5 \%$ of the error at the optimal gain. Considering a relative torque error of only $2 \%-8 \%$ of desired torque at the optimum, a $5 \%$ increase on top is rather insignificant.

\subsection{Conclusions}

This study investigates the existence of an optimal iterative learning gain of desired motor position for torque tracking in ankle exoskeleton during walking under velocity controlled motor operational mode. Theoretical analysis with simplified system model identifies the optimal gain as the inverse of transmission stiffness relative to the motor side. Walking experiments supports the hypothesis with a shallow slope of changes in tracking errors around the neighborhood of the theoretical optimal gain. Results of this study suggests a recommended iterative learning gain range that is [50\% 200\%] of the hypothesized optimum with limited knowledge of the complicated system dynamics. 


\section{Chapter 6}

\section{Passive Series Stiffness Optimization}

\subsection{Introduction}

Previous studies revealed the effectiveness of a torque tracking structure made of the combination of model-free, integration-free feedback control and iterative learning in lower-limb exoskeleton systems [92, 60, 61]. With this control architecture, fairly strong interactions were also observed experimentally in torque tracking between the device passive stiffness and high-level controller that determines desired torques, which suggest potential room for torque tracking performance to be improved. This study explores the possibility to further improve torque control performance in lower-limb exoskeletons under fixed control objectives, with limited knowledge of the complete system dynamics, by optimizing the passive stiffness of actuators.

Traditionally, robot actuation has been made as stiff as possible for improved precision, stability and bandwidth of position control [146, 31]. Compliant actuators have been widely used in wearable devices during the past two decades due to its ability to improve human safety and comfort $[32,25-27,147]$ and reduce shock loads $[31,76$, 
147]. In the realization of compliant actuation, passive compliance is usually involved due to its improved safety, increased interaction control bandwidth and its ability of energy storage $[148,76,147]$. Series elastic actuators are one type of actuators with passive compliance that have gained great popularity due to their enhanced force control performance and improved human experience [31, 33, 32, 83, 84, 149].

The stiffness of elastic element is crucial in design of series elastic actuators since it highly affects the performance of the device in various applications [150]. Multiple criteria have been employed in the selection of passive compliance. Higher stiffness in spring increases the open-loop bandwidth of an actuator and is more desirable for the purpose of increasing force bandwidth [97]. Lower stiffness, however, increases the capacity of energy storage in actuators [97]. Many devices also select series stiffness by balancing torque/force requirements, device geometry and device weight $[6,90,7]$. Multiple works discussed the optimization of passive stiffness in series elastic actuators for fixed applications from the perspective of energy consumption [150-152]. Some aimed to optimize total energy consumption by general analysis $[153,151,152]$, matching the natural frequency of the actuator to the desired motion [154], or matching system source and load impedance [155]. Some tried to optimize application peak power capacity [150].

There were some previous works that addressed the effects of passive stiffness of series elastic actuators on torque tracking performance, most of which were by means of theory $[152,147]$, simulation or benchtop tests without realistic experimental conditions [155]. [88] briefly discussed the role of series elastic actuator passive stiffness in torque tracking, which states that too high and too low stiffness both worsen its control performance. It would be beneficial to investigate thoroughly the existence of the optimum in passive stiffness and its exact value by means of both theoretical analysis and realistic experiments. 
This study attends to the optimization of the passive series stiffness in lower-limb exoskeletons in the context of torque tracking during human walking, under the popular impedance based high level controller $[6,79,7]$ using both theory and experiments. This type of high level controller adjusts the torque applied to human body according to joint position and realizes a desired quasi-stiffness, which is defined as the slope of the desired torque-angle relationship [156]. It is especially popular among locomotion related robots due to the high repeatability and ease of energy input manipulation to human body $[6,79,7]$. The results are expected to guide application-specific hardware design of lower limb wearable robots used in gait assistance or rehabilitation.

\subsection{Methods}

To investigate the influence of passive stiffness on torque tracking performance in series elastic actuators, we did a case study on a tethered ankle-foot exoskeleton driven by a uni-directional Bowden cable [7]. We developed a simplified model of the exoskeleton system, base on which we made hypotheses that relate torque tracking performance, actuator passive stiffness and control parameters. We then conducted walking experiments while one subject wearing the exoskeleton on the right foot. Eight different desired torque-angle relationships were implemented. Each was tested in combination with six passive stiffness configurations by switching the series spring in the transmission sub-system of the device. Every combination of desired and passive stiffness values, which we denote as one 'stiffness combination' hereinafter in this chapter, incurred multiple walking experiments on treadmill while the subject wore the device with different control gains. Comparison of the resulting torque tracking errors of these experiments identifies the best-observed tracking performance and corresponding control parameters of the current stiffness combination, which serve as the estimates of the actual optimal performance and control parameters. The observed optima were then 
investigated against the values of desired and passive stiffnesses to test and validate the hypotheses.

\subsubsection{Exoskeleton system and simplified model}

The exoskeleton testbed system was the same as the one used in Chapter 4 and 5, which comprised of an off-board real-time control module and geared electric motor, a unidirectional Bowden cable transmission with a series spring, and an ankle exoskeleton frame that interfaced with the human foot and shank (Fig. 4.1 and 5.1).

To facilitate and ease mathematical analysis and make prediction for passive stiffness optimum for a pre-determined desired quasi-stiffness, the same simplifications were made in modeling the system as Section 5.2.1.

\subsubsection{Controllers}

\section{Low Level Control: Proportional Control + Damping Injection}

Previous work identified model-free, integral-action-free feedback control compensated by iterative learning as the most effective controller for lower-limb exoskeletons torque tracking during walking $[60,61]$. To simplify the testing of tracking performance, only the feedback part of the controller was used in theoretical analysis and experiments of this study:

$$
\begin{aligned}
& \dot{\theta}_{p, \text { des }}=\underbrace{-K_{p} \cdot e \tau}_{\text {Proportional Control }}+\underbrace{-K_{d} \cdot \dot{\theta}_{p}}_{\text {Damping Injection }} \\
& \dot{\theta}_{m, \text { des }}=N \dot{\theta}_{p, \text { des }}
\end{aligned}
$$


This controller has two parts: proportional control and damping injection. In this formulation, $e_{\tau}=\tau-\tau_{d e s}$ is torque error, $\tau$ is measured exoskeleton torque, $\tau_{d e s}$ is desired exoskeleton torque. Damping injection on motor velocity is used instead of derivative control over torque errors to reduce the effect of measurement noise, which is more severe in the latter since torque is measured and transmitted in analog form while motor position is in digital and nonlinearities is present in Bowden cable transmission. $K_{p}$ is a proportional gain and $K_{d}$ is a damping gain. The motor runs in velocity mode with the desire motor output pulley velocity $\dot{\theta}_{p, d e s}$ converted to desired motor velocity $\dot{\theta}_{m, d e s}$ before asserted. $N$ is the gear ratio of the motor.

\section{High Level Control: Desired Quasi-Stiffness}

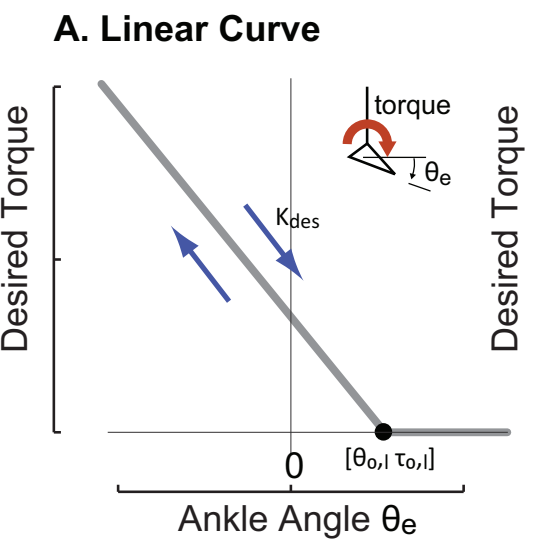

\section{B. Piece-wise Linear Curve}

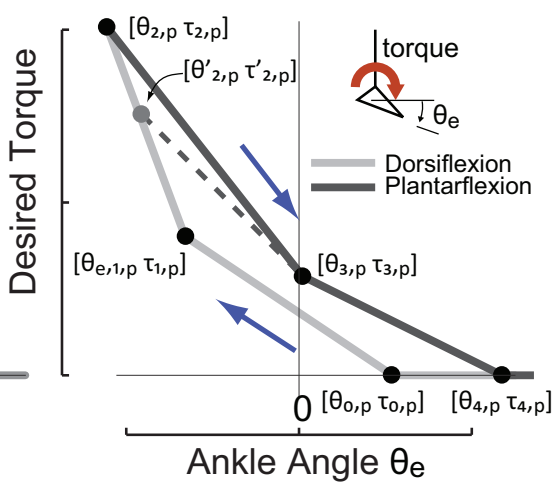

Figure 6.1: The two types of ankle angle based high-level desired torque curves imposed in experiments to realize different desired quasi-stiffness profiles. (A) High-level controller I commands desired torque that is linearly proportional to exoskeleton joint angle $\theta_{e}$ defined by anchor point $\left[\theta_{0, l} \tau_{0, l}\right]$ and desired quasi-stiffness $K_{d e s}$. (B) High-level controller II commands desired torque that is piece-wise linearly proportional to joint angle $\theta_{e}$ with four phases defined by anchor points $\left[\theta_{0, p} \tau_{0, p}\right],\left[\theta_{1, p} \tau_{1, p}\right],\left[\theta_{2, p} \tau_{2, p}\right],\left[\theta_{3, p} \tau_{3, p}\right]$ and $\left[\theta_{4, p} \tau_{4, p}\right]$. In both cases, desired torque is lower-bounded at zero.

Two types of ankle angle based desired torque curves were used to realize different desired quasi-stiffnesses. One was a linear torque versus ankle angle curve as shown in 
Fig. 6.1.A and expressed in Eq. (6.2).

$$
\begin{aligned}
\tau_{d e s} & =S \cdot K_{d e s, 0} \cdot\left(\theta_{e}-\theta_{0, l}\right) \\
& =-K_{d e s} \cdot\left(\theta_{e}-\theta_{0, l}\right) \\
\tau_{d e s} & =\max \left(\tau_{d e s}, 0\right)
\end{aligned}
$$

where $\left(\theta_{0, l}, \tau_{0, l}\right)$ is an anchor node in torque-angle space and $K_{d e s}$ is the resulted desired stiffness. $S$ is a scaling factor on the unit curve with desired quasi-stiffness of $K_{d e s, 0}$ to get different values of desired quasi-stiffness.

Another type of curve tested was a piece-wise linear torque-angle curve with the format of Eq. (6.3) as shown in Fig. 6.1.B.

$$
\begin{aligned}
\tau_{d e s}= & S \cdot \frac{\tau_{i, p}-\tau_{i-1, p}}{\theta_{i, p}-\theta_{i-1, p}} \cdot\left(\theta_{e}-\theta_{i-1, p}\right) \\
\tau_{d e s}= & \max \left(\tau_{d e s}, 0\right) \\
& \text { for } i=\{1,2,3,4\}
\end{aligned}
$$

Here, $\left(\theta_{i, p}, \tau_{i, p}\right)$ defines a node in torque-angle space (Fig. 6.1.B). $i$ denotes the phase number on the curve the gait is currently in. The node $\left(\theta_{2, p}, \tau_{2, p}\right)$ marked the transition from the dorsiflexion phase, in which ankle velocity was negative, to the plantarflexion phase, in which ankle velocity was positive. Since the exact transition point varied on each stride, we used the angle and torque at the moment of transition, $\left(\theta_{2, p}^{\prime}, \tau_{2, p}^{\prime}\right)$, when calculating desired torque in the first portion of Plantarflexion, i.e., phase 3 of the curve.

\subsubsection{Theoretical Analysis and Hypotheses}

We conducted theoretical analysis based on the analytic expressions of the testbed system dynamics, desired torque, and torque controller and made hypotheses about the 
optimum of passive stiffness of series elastic actuators in lower-limb ankle exoskeletons and the interactions between optimal gains, desired stiffness and passive stiffness.

\section{Optimal Passive Stiffness}

To further ease the theoretical analysis for the prediction of passive stiffness optimum in series elastic actuators, we modeled the assisted walking with the ankle exoskeleton as an oscillator. Oscillators are efficient modeling tools in biological and physical sciences due to their capability to synchronize with other oscillators or with external driving signals $[157,158]$. Multiple efforts have been made towards improving the synchronization capabilities of nonlinear oscillators by adapting their frequencies [158]. The concept has been introduced and employed in locomotion to either improve the identification of central pattern generator parameters [159, 157, 160-162], to better estimate state measurements [163, 164, 92], or to help with controller design [165, 166] by exploiting the cyclic behavior of walking. Therefore, we model various states of walking as synchronized oscillations. This method disburden our analysis from dealing with complicated human-robot interactive dynamics, focus on the resulting states like ankle kinematic profile and required motor position profile that are close to be periodical, and significantly simplified our analysis. However, neglecting of step-to-step variations in practical cases does cause potential deviation of experimental results from theoretical hypotheses.

With proportional control and damping injection in Eq. (6.1) used for torque tracking,

$$
\begin{aligned}
\dot{\theta}_{p, d e s} & =-K_{p} e \tau-K_{d} \dot{\theta}_{p} \\
& =-K_{p}\left[K_{t}\left(\theta_{p} R-\theta_{e}\right)+K_{d e s}\left(\theta_{e}-\theta_{0}\right)\right]-K_{d} \dot{\theta}_{p}
\end{aligned}
$$

Due to the employment of a high-speed real-time controller and a high-acceleration servo motor, desired motor velocity is enforced rapidly, based on which we make the 
simplification of immediate motor velocity enforcement, i.e.,

$$
\dot{\theta}_{p}=\dot{\theta}_{p, d e s}
$$

Combining Eq. (6.5) with a linear approximation of desired torque curves, including those expressed by Eqs. (6.2) and (6.3), in the form of

$$
\tau_{d e s}=-K_{d e s}\left(\theta_{e}-\theta_{0}\right)
$$

we have

$$
\left(1+K_{d}\right) \dot{\theta}_{p}=-K_{p}\left[K_{t}\left(\theta_{p} R-\theta_{e}\right)+K_{d e s}\left(\theta_{e}-\theta_{0}\right)\right]
$$

in which $\theta_{0}$ is maximum joint position for the device to exert torque on the human ankle, i.e., the intersection of torque-angle relationship with the angle axis.

Modeling exoskeleton-assisted walking after stabilization as an oscillation process made of $N$ sinusoidal waves of the same frequency $F$, we get a profile of the ankle angle in the form of

$$
\theta_{e}=c+\sum_{n=1}^{N} d_{n} \cdot \exp \left(j 2 \pi F t+\beta_{n}\right),
$$

where $c$ is an constant denoting the offset of the profile on torque axis, $d_{n}$ and $\beta_{n}$ are the magnitude and phase shift of the $n^{\text {th }}$ sinusoidal wave, and $t$ represents the time elapsed within one stride since heel strike. The corresponding stabilized motor position should also oscillate with the same frequency. We can therefore construct a stabilized motor position by equal number of sinusoidal waves with the same phase shifts in the form of

$$
\theta_{p}=e+\sum_{n=1}^{N} f_{n} \cdot \exp \left(j 2 \pi F t+\beta_{n}\right)
$$

in which $e$ is a constant and $f_{n}$ is a complex number. Substituting Eq. (6.8) and (6.9) 
into Eq. (6.7), we get Eq. (6.10).

$$
\begin{array}{r}
{\left[\left(1+K_{d}\right) j 2 \pi F+K_{p} K_{t} R\right] \sum_{n=1}^{N} f_{n} \cdot \exp \left(j 2 \pi F t+\beta_{n}\right)} \\
=-K_{p} K_{t} \operatorname{Re}-K_{p}\left(K_{d e s}-K_{t}\right) c+K_{p} K_{d e s} \theta_{0} \\
-K_{p}\left(K_{d e s}-K_{t}\right) \sum_{n=1}^{N} d_{n} \cdot \exp \left(j 2 \pi F t+\beta_{n}\right)
\end{array}
$$

Equating the coefficients of the various sinusoidal waves and the offset, we have

$$
f_{n}=\frac{-K_{p}\left(K_{d e s}-K_{t}\right)}{\left(1+K_{d}\right) j 2 \pi F+K_{p} K_{t} R} d_{n}
$$

and

$$
e=-\frac{K_{d e s}-K_{t}}{K_{t} R} c+\frac{K_{d e s}}{K_{t} R} \theta_{0}
$$

Motor position profile in Eq. (6.9) can thus be expressed in terms of the ankle position profile and the controller as

$$
\begin{aligned}
\theta_{p}= & -\frac{K_{d e s}-K_{t}}{K_{t} R} c+\frac{K_{d e s}}{K_{t} R} \theta_{0} \\
& +\frac{-K_{p}\left(K_{d e s}-K_{t}\right)}{\left(1+K_{d}\right) j 2 \pi F+K_{p} K_{t} R} \sum_{n=1}^{N} d_{n} \cdot \exp \left(j 2 \pi F t+\beta_{n}\right) .
\end{aligned}
$$

Combining the oscillator assumption with Eq. (6.7), we get the expression of the torque error as

$$
\begin{aligned}
& e_{\tau} \\
& =\tau-\tau_{d e s} \\
& =K_{t}\left(\theta_{p} R-\theta_{e}\right)+K_{d e s}\left(\theta_{e}-\theta_{0}\right) \\
& =K_{t} R \theta_{p}+\left(K_{d e s}-K_{t}\right) \theta_{e}-K_{d e s} \theta_{0} \\
& =\left(K_{d e s}-K_{t}\right) \frac{\left(1+K_{d}\right) j 2 \pi F}{\left(1+K_{d}\right) j 2 \pi F+K_{p} K_{t} R} \sum_{n=1}^{N} d_{n} \cdot \exp \left(j 2 \pi F t+\beta_{n}\right) .
\end{aligned}
$$


It is clear that without considering the control gains, asserting

$$
K_{d e s}-K_{t}=0
$$

will minimize torque tracking error. Therefore, we make the following hypothesis:

Hypothesis 1. In lower-limb exoskeletons, the optimal passive stiffness of the series elastic actuator for torque tracking is

$$
K_{t, o p t}=K_{d e s}
$$

\section{Relationship between Torque Tracking Performance and the Difference of Desired and Passive Stiffnesses}

Another factor that limits torque tracking performance is the inability of the proportional gain to increase indefinitely.

Reformatting Eq. (6.14), we get

$$
\begin{aligned}
& e_{\tau}= \\
& \frac{j 2 \pi F}{\frac{K_{p}}{1+K_{d}} K_{t} R+j 2 \pi F}\left(K_{d e s}-K_{t}\right) \sum_{n=1}^{N} d_{n} \cdot \exp \left(j 2 \pi F t+\beta_{n}\right) .
\end{aligned}
$$

It is clear that when the passive stiffness is fixed but does not match the desired one, i.e., $K_{t}-K_{d e s} \neq 0$, with the same step frequency $F$ and angle profile $\sum_{n=1}^{N} d_{n} \cdot \exp (j 2 \pi F t+$ $\left.\beta_{n}\right)$, torque tracking error $e \tau$ is inversely proportional to $\frac{K_{p}}{1+K_{d}}$.

Meanwhile, combining the controller in Eq. (6.4) and the assumption of perfect 
motor velocity tracking in Eq. (6.5), we have

$$
\dot{\theta}_{p}=-\frac{K_{p}}{1+K_{d}} e \tau
$$

Differentiating the expression of applied torque in Eq. (4.6), we get

$$
\dot{\tau}=K_{t}\left(\dot{\theta}_{p} R-\dot{\theta}_{e}\right)
$$

Therefore, the time derivative of torque error is

$$
\begin{aligned}
\dot{e}_{\tau} & =\dot{\tau}-\dot{\tau}_{d e s} \\
& =K_{t}\left(\dot{\theta}_{p} R-\dot{\theta}_{e}\right)+K_{d e s} \dot{\theta}_{e} \\
& =-K_{t} R \frac{K_{p}}{1+K_{d}} e_{\tau}-K_{t} \dot{\theta}_{e}+K_{d e s} \dot{\theta}_{e}
\end{aligned}
$$

which is a first order dynamics created by feedback control with an effective proportional gain of

$$
\frac{K_{t} \cdot R \cdot K_{p}}{1+K_{d}}
$$

and a time constant of

$$
\varsigma=\frac{1+K_{d}}{K_{t} \cdot R \cdot K_{p}}
$$

However, this dynamics does not exist independently but interacts with the human body in parallel. Therefore, in practical cases, oscillations increase when effective proportional gain increases, which impairs torque tracking performances eventually and causes discomfort or injury to the human body. In our study, motor speed limit was never hit. Thus we suspected that there is a fixed torque tracking bandwidth limit that is dependent on the combined interactive dynamics of motor, motor drive, transmission and human body. This bandwidth limit results in a fixed maximum commanded change rate of torque error, $\dot{e} \tau$,max, which corresponding to the best tracking performance regardless of the passive stiffness of the system. We therefore proposed the following conjecture: 
Conjecture 1. Assisted human walking with a lower-limb exoskeleton experiences a fixed maximum commanded tracking rate of torque error, $\dot{e}_{\tau, \text { max }}$, which limits the tracking performance of the system.

In practical cases, Eq. (6.19) can be further simplified. First, to realize real-time torque tracking, the motor velocity should be a lot faster than device joint velocity, i.e., $\dot{\theta}_{p} \gg \dot{\theta}_{e}$, which combines with the fact that $R=2.5$ results in the following fact about Eq. (6.18):

$$
\dot{\tau} \approx K_{t} R \dot{\theta}_{p}
$$

Successful torque tracking also means a fast changing rate of actual torque compared to the desired torque, $\dot{\tau} \gg \dot{\tau}_{d e s}$, which leads to the results of dominance of applied torque changing rate in torque error changing rate, i.e.,

$$
\dot{e}_{\tau} \approx \dot{\tau}
$$

Therefore, Eq. (6.19) can be estimated as:

$$
\dot{e}_{\tau} \approx-K_{t} R \frac{K_{p}}{1+K_{d}} e_{\tau}
$$

This is equivalent to say that $\lambda$ in Eq. (6.25) is small and neglectable and $\frac{K_{p}}{1+K_{d}}$ and $K_{t}$ are inversely proportional to each other. The application of Conjecture 1 in this case results in a fixed time constant $\frac{1+K_{d}}{K_{t} R K_{p}}$ at optimal control conditions. Together with the assumption of a rather constant step frequency $F$ and a constant angle profile $\sum_{n=1}^{N} d_{n} \cdot \exp \left(j 2 \pi F t+\beta_{n}\right)$, torque error as expressed by Eq. (6.16) is proportional to the difference between passive and desired stiffness values, i.e.,

$$
e_{\tau, o p t} \propto K_{d e s}-K_{t},
$$


which then leads to the hypothesis below.

Hypothesis 2. The root-mean-squared torque tracking errors under optimal feedback control conditions are proportional to the absolute difference between the desired and passive stiffness values, i.e.,

$$
\left\|e_{\tau, o p t, R M S}\right\| \propto\left\|K_{d e s}-K_{t}\right\|
$$

\section{Interactions between Optimal Control Gains and Passive Stiffness}

Dynamics in Eq. (6.19) directly leads to a relationship between $K_{p}$ and $K_{t}$ :

$$
K_{p}=\frac{\left(K_{d e s} \dot{\theta}_{e}-\dot{e} \tau\right)\left(1+K_{d}\right) R^{-1} e_{\tau}^{-1}}{K_{t}}-\dot{\theta}_{e}\left(1+K_{d}\right) R^{-1} e_{\tau}^{-1}
$$

which can be simplified under the same desired torque-angle relationship, i.e., $K_{d e s}$. Previous study shows an root-mean-squared tracking error of $<8 \%$ the peak desired torque under proportional control and damping injection $[60,61]$, which is expected to be improvable with better control parameters and different curve types. This suggests that under optimal torque tracking conditions, the actual applied torque profiles with the same $K_{d e s}$, are expected to be fairly constant regardless of the value of passive stiffness $K_{t}$. Meanwhile, although the exact exoskeleton-human interactive dynamics is difficult to identify, we expect the relationship between applies torque and resulting human ankle kinematics to obey of Newton's law. Therefore, a fairly constant torque profile from the exoskeleton, when applied to the same subject under the same walking speed and step frequencies with low variance, should produce rather constant human and device joint kinematics, $\theta_{e}$ and $\dot{\theta}_{e}$. Therefore, the extreme device joint velocity that would produce the highest torque error rate with fixed control gains and push the con- 
trolled system to its bandwidth limit, $\theta_{e, e x t}$, does not vary significantly across different passive stiffness conditions. Similar assumptions can be made about the extreme torque error $e_{\tau, \text { ext }}$. Meanwhile, gain of the less dominant damping injection control part, $K_{d}$, have been observed to be upper-bounded by the appearance of motor juddering in our experiments at $K_{d, \max }=0.6$ for various stiffness combinations. The approximated invariance of $\theta_{e, \text { ext }}$ and $K_{d, \max }$, combined with a fixed $\dot{e}_{\tau, \text { max }}$ as assumed by Conjecture 1 , lead to the following hypothesis.

Hypothesis 3. With the same desired torque-angle curve, thus the same $K_{d e s}$, the optimal proportional gain $K_{p, o p t}$ is related to the passive stiffness $K_{t}$ by

$$
K_{p, o p t}=\frac{\sigma}{K_{t}}+\lambda
$$

in which $\sigma$ is dependent on the desired stiffness $K_{d e s}$ and can be expressed as

$$
\sigma=\left(K_{d e s} \dot{\theta}_{e, e x t}-\dot{e}_{\tau, \max }\right)\left(1+K_{d, \max }\right) R^{-1} e_{\tau, \text { ext }}^{-1}
$$

and the constant $\lambda$ is

$$
\lambda=-\dot{\theta}_{e, e x t}\left(1+K_{d, \max }\right) R^{-1} e_{\tau, e x t}^{-1}
$$

To ease later presentation, we label the value $\sigma$ here as $\mathbf{K}_{\mathbf{p}}-\mathbf{K}_{\mathbf{t}}$ coefficient hereinafter.

To realize torque tracking, proportional control is always dominant over damping 
injection. Therefore, Eq. (6.17) can be simplified as

$$
\dot{\theta}_{p, d e s} \approx K_{p} e \tau
$$

and accordingly, Eq. (6.22) becomes

$$
\dot{e}_{\tau} \approx-K_{t} R K_{p} e_{\tau}
$$

which suggests that we can further simplify Hypothesis 3 with an approximated inverse proportional relationship between the optimal $K_{p}$ and $K_{t}$. Therefore, the following corollary can be made.

Corollary 1. For a fixed desired torque-angle relationship, i.e, $K_{d e s}$, when the passive stiffness of the series elastic actuator of the device is changed from $K_{t, o l d}$ to $K_{t, n e w}$, an estimate of the new optimal proportional control, $K_{p, n e w}$, can be achieved by

$$
K_{p, \text { new }} \approx \frac{K_{p, \text { old }} \cdot K_{t, \text { old }}}{K_{t, \text { new }}}
$$

in which $K_{p, o l d}$ is the optimal proportional control gain at $K_{t, o l d}$.

Although multiple approximations have been made in the derivation of this corollary, which causes inaccuracies in this estimation, it can be used to set a starting point of proportional control gain tuning when system passive stiffness is changed with only 
the knowledge of the old and new passive stiffness values.

\section{Relationship between $K_{p}-K_{t}$ Coefficient and Desired Stiffness}

Furthermore, combining Eq. (6.19), (6.25) and (6.27) at optimal control conditions, we have

$$
\begin{aligned}
\dot{e} \tau, \text { max } & =-\sigma R\left(1+K_{d, \text { max }}\right)^{-1} e \tau+K_{d e s} \dot{\theta}_{e} \\
& =-\sigma R\left(1+K_{d, \text { max }}\right)^{-1}\left[\tau+K_{d e s}\left(\theta_{e}-\theta_{0}\right)\right]+K_{d e s} \dot{\theta}_{e}
\end{aligned}
$$

which means

$$
\sigma=\frac{\left(1+K_{d, \max }\right) K_{d e s} \dot{\theta}_{e}-\dot{e} \tau, \max }{R\left[K_{d e s}\left(\theta_{e, e x t}-\theta_{0}\right)+\tau\right]}
$$

With relatively invariant extreme ankle velocity values, $\theta_{e, \text { ext }}(t)$, and torque error values $\dot{e} \tau, \max$, across different desired stiffness, at a time of similar measured torque $\tau$, the following hypothesis can then be drawn.

Hypothesis 4. The $K_{d}-K_{t}$ coefficient in Eq. (6.25) is related to the desired quasistiffness $K_{\text {des }}$ by

$$
\sigma=\frac{\varsigma \cdot K_{d e s}+\delta}{K_{d e s}+\xi}
$$

in which $\varsigma, \delta$ and $\xi$ are constant parameters, and

$$
\delta=\frac{\left(1+K_{d, \max }\right)}{R\left(\theta_{e, \max }-\theta_{0}\right)} \dot{e} \tau, \max
$$

is linearly related to the hypothesized maximum commanded torque change rate $e_{\tau, \max }$. 


\subsubsection{Dynamic Complications not Featured}

Multiple assumptions and approximations of the system dynamics were made to simplify our theoretical analysis in forming the hypotheses. Complications of the system that were not captured by the models we used are listed below.

$\diamond$ There are frictions and stictions in the Bowden cable transmission, which are also time-varying depending on the shape of the cable, conditions of the inner rope and conduit, tension in the rope and also velocity of the relative motion between the rope and conduit.

$\diamond$ The transmission stiffness is nonlinear due to the existence of synthetic rope in Bowden cable, which is stretchy under a small tension and stiffer under larger. This stiffness is also subject to changes due to the slow-stretching of the rope and step-to-step gait variation.

$\diamond$ The lever arm of series spring force with respect to the device joint is not a constant.

$\diamond$ There is a communication delay and rise time during enforcement of the desired velocity. Therefore, there can never be perfect tracking.

Due to step-to-step variation, even after stabilization, the assisted walking is not an exactly periodical oscillatory process as assumed.

All these unfeatured complications in system dynamics can cause reality to deviate from the theoretical analysis to some extent. To ensure the guidance for hardware and controller design we provide to be meaningful practically, we conducted walking experiments to test and validate our hypotheses. 


\subsubsection{Testbed System Configurations}

We tested the hypotheses with different springs on a tethered ankle exoskeleton comprised of an off-board real-time control module and geared electric motor, a uni-directional Bowden cable transmission with a series spring, and an exoskeleton frame that interfaced with the human foot and shank (Fig. 4.1 and 5.1).

\subsubsection{Experimental Methods}

Since the purpose of experiments in this study was to quantify not the human reaction but the torque tracking performance of various hardware and control conditions, only one healthy subject $(\mathrm{N}=1$, female, 32 years, $1.65 \mathrm{~m}, 56 \mathrm{~kg})$ was involved. The subject walked on a treadmill with a fixed speed of $1.25 \mathrm{~m} / \mathrm{s}$ with a self-paced step frequency while wearing the tethered ankle exoskeleton on the right leg in all experiments. All experimental protocols were approved by Carnegie Mellon University IRB.

For the ease of readability, this chapter uses Newton-meter, degree and meter as the corresponding units for measurements of torque, angle and distance.

\section{Desired Torque-Angle Curves and Evaluation of Their Desired Stiffness Values}

To test the hypotheses, eight desired quasi-stiffnesses, i.e., torque versus ankle angle relationship, were implemented, including three linear and five piece-wise linear curves. A unit linear curve ( $S=1$ in Eq. 6.2) was defined by parameter values in Table 6.1. The three linear curves, L1, L2 and L3, were achieved by scaling the unit curve on the desired torque axis with factors of $0.4,1$ and 1.7 respectively. A unit piece-wise linear curve ( $S=1$ in Eq. 6.3) was defined by the parameter values listed in Table 6.2. Five piece-wise linear curves, P1, P2, P3, P4 and P5, were then achieved by scaling the unit 
curve with factors $0.4,0.7,1,1.3$ and 1.7 . The resulting desired torque versus ankle angle curves are shown in Fig. 6.2.

Table 6.1: Linear unit curve parameter values

\begin{tabular}{llll}
\hline Param & Value & Param & Value \\
\hline$\left[\theta_{0, l} \tau_{0, l}\right]$ & {$[-2,0]$} & $K_{d e s, 0}$ & 5 \\
\hline
\end{tabular}

Table 6.2: Piece-wise linear unit curve parameter values

\begin{tabular}{llll}
\hline Param & Value & Param & Value \\
\hline$\left[\theta_{0, p} \tau_{0, p}\right]$ & {$[-2,0]$} & {$\left[\theta_{1, p}, \tau_{1, p}\right]$} & {$[-8,20]$} \\
{$\left[\theta_{2, p}, \tau_{2, p}\right]$} & {$[-12,50]$} & {$\left[\theta_{3, p}, \tau_{3, p}\right]$} & {$[0,12.5]$} \\
{$\left[\theta_{4, p}, \tau_{4, p}\right]$} & {$[8,0]$} & & \\
\hline
\end{tabular}

A. Linear Curves

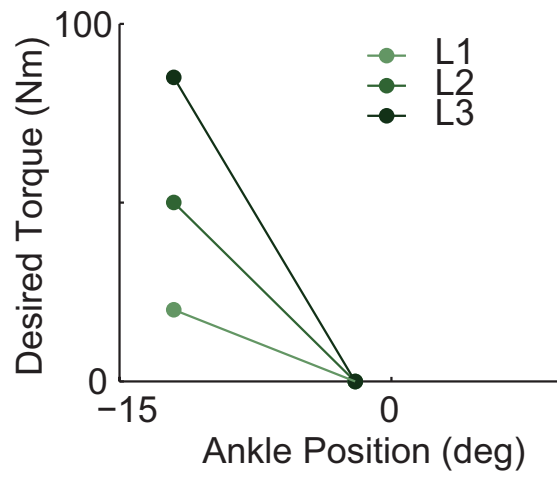

B. Piece-wise Linear Curves

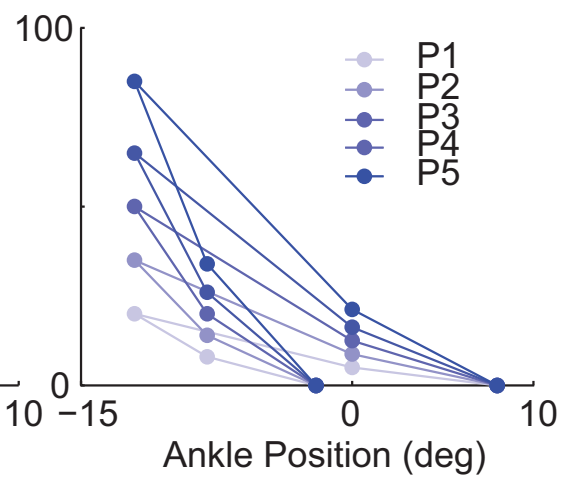

Figure 6.2: All eight tested desired torque versus ankle angle curves used to realize different desired quasi-stiffness values. (A) Three linear curves achieved by scaling unit curve defined by Table 6.1 with scaling factors of $0.4,1$ and 1.7. (B) Five piece-wise linear curves by achieved by scaling unit curve defined by Table 6.2 with scaling factors of $0.4,0.7,1,1.3$ and 1.7.

Calculation of desired quasi-stiffness values are different for linear and piece-wise cases. For linear curves, the values of L1, L2 and L3 can be easily evaluated as 2, 5 and $8.5 \mathrm{Nm} / \mathrm{deg}$ respectively. This set spans a range of $6.5 \mathrm{Nm} / \mathrm{deg}$ with a maximum that is 4.25 times the minimum. For the case of piece-wise linear curves, we primarily used the desired stiffness values of each of the four phases and investigated different phases separately. The desired quasi-stiffness values in this case ranges from 0.625 to 12.75 Nm/deg. 
Table 6.3: List of springs used in experiments with assigned ID

\begin{tabular}{c|cccccc}
\hline $\begin{array}{c}\text { Passive Stiffness } \\
\text { ID }\end{array}$ & S1 & S2 & S3 & S4 & S5 & S6 \\
\hline Spring Part No. & DWC-148M-13 & DWC-162M-12 & DWC-187M-12 & DWC-225M-13 & DWC-250M-12 & No Spring \\
Length (m) & 0.0635 & 0.0508 & 0.0508 & 0.0635 & 0.0508 & - \\
$\begin{array}{c}\text { Spring Rate } \\
\left(\mathbf{N} / \mathbf{m} \times 10^{3}\right)\end{array}$ & 15.1 & 27.5 & 50.1 & 103.1 & 235.7 & - \\
Max Load (N) & 413.7 & 578.3 & 778.4 & 1641.4 & 2246.4 & - \\
\hline
\end{tabular}

\section{Realization of Different Passive Stiffness and Evaluation of Their Values}

For each of the desired stiffness profile defined by a torque-angle relationship, six passive series stiffness values of the transmission system were realized by changing the series spring of the ankle exoskeleton (Fig. 5.1.A), the same way as in Chapter 5. The list of springs used and their corresponding properties are available in Table 6.3.

The effective passive stiffness values of various spring configurations, $K_{t}$, are evaluated the same way as Chapter 5. Fig. 6.3 shows the plots of passive walking sessions for different spring configurations.

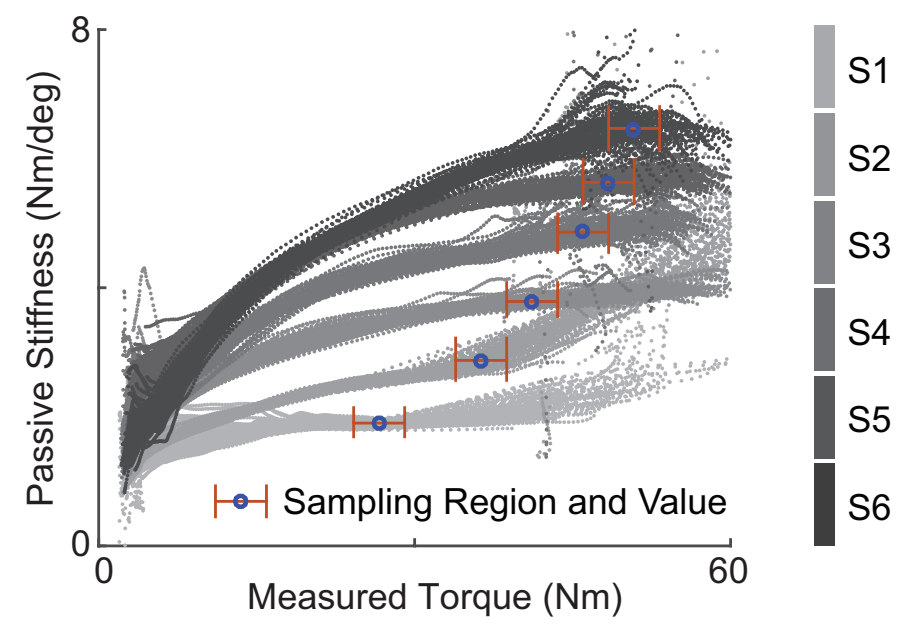

Figure 6.3: Instantaneous passive stiffness values of passive walking sessions are plotted against the measured torques for various spring configurations, one session for each. One experiment session consists of one-hundred steps with motor position fixed. The stablized passive stiffness value for one session is defined as the median of the values over a stabilized region. The effective passive stiffness of the one stiffness configuration is defined as the mean of stabilized stiffness values across multiple sessions. 


\section{Definition of Difference in Desired and Passive Stiffness Values}

The difference between the desired and passive stiffnesses is an important index since Hypotheses 1 and 2 state that the optimal passive stiffness for torque tracking equals the desired quasi-stiffness and torque errors are closely related to the difference between the two. In analyzing our experimental results, this value is defined as the algebraic difference between the desired and passive values, i.e., $K_{t}-K_{d e s}$.

\section{Experiment Procedures}

The key to be able to compare the influence of passive stiffness on torque tracking performance under a fixed desired quasi-stiffness is to evaluate the 'best' tracking performance under each passive stiffness configuration. We did so by experimentally evaluate the tracking errors of multiple experimental sessions, each with different feedback control gains. The lowest error across these trials was then assigned as the estimate of the actual optimal performance with this passive stiffness.

For each combination of desired and passive stiffnesses, the initial session had fairly low proportional and damping gains. The gains were gradually increased across trials until perceptible oscillations were detected with maximum damping gain. Depending on the initial gains and step sizes of gain tuning, number of trials varies for each stiffness combination. Sometimes, the gains are lowered in the final sessions to achieve better gain tuning resolution. On average, around ten trials were conducted for each stiffness combination.

Identification of the best torque tracking performance for a specific desired and passive stiffness combination is crucial. The step-wise root-mean-squared (RMS) torque tracking errors averaged over the one hundred steady steps for each experimental trial was calculated as its performance indicator. For each combination of desired and pas- 
sive stiffnesses, the RMS error values of all trials with different gains were compared.

The lowest of them was recorded as the estimate of optimal torque error for the corresponding stiffness combination. The control gains of the corresponding data set were recorded as the estimates of optimal control gains.

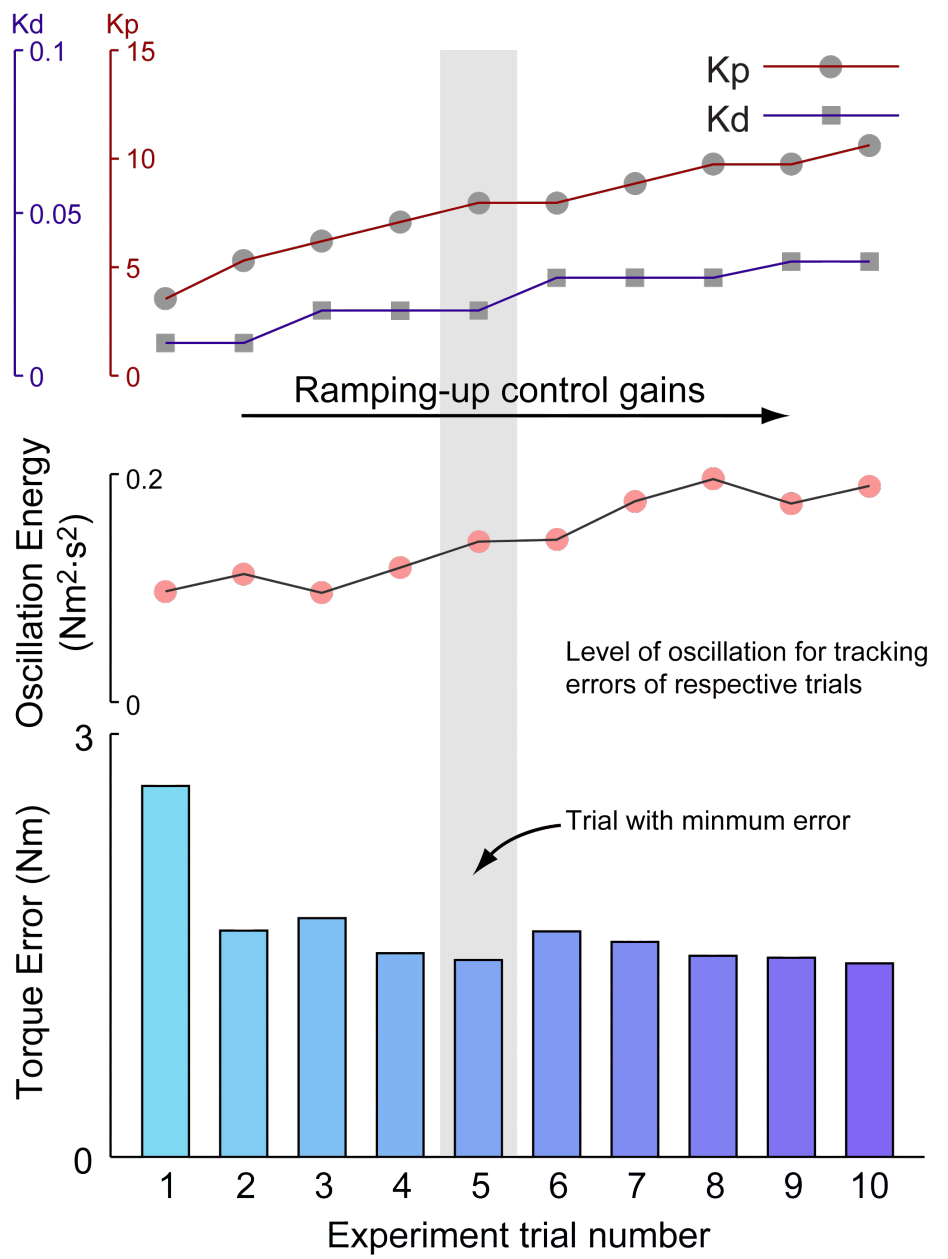

Figure 6.4: Demonstration of the experimental procedure for one example combination of desired and passive stiffness. For stiffness combination, the first experiment session starts with a moderate set of proportional and damping injection gains. For the subsequent sessions, each of which includes at least one-hundred steady strides, the proportional gain was firstly increased until oscillations became noticeable. Then, damping injection gain was increased until oscillations were reduced to be imperceptible. Proportional and damping injection were then increased alternatively until perceptible oscillations seen with maximum damping injection gain. The root-mean-squared torque tracking errors of each session was then calculated and compared against one another. The best-performed session was identified with the lowest error and its tracking error and control gains are then registered as the estimates of optimal tracking performance and optimal control gains for this specific stiffness combination. The level of oscillation for each session is also displayed. It is seen that with the increase of control gains, torque tracking errors first drop, but later increase due to increasing oscillations. 
Then, the lowest torque tracking errors and the control gains of corresponding experimental sessions for all stiffness combinations were investigated against the difference between desired and passive stiffness values to test the hypotheses. This process is demonstrated in Fig. 6.4, which presents the control gains, experimental sequence, resulting RMS torque errors and the corresponding oscillation levels of measured torques for each data set with one combination of desired and passive stiffness.

\section{Level of Oscillation}

The level of oscillation included in Fig. 6.4 is an indicator defined to show the amount of oscillations in the control results of each experiment session. As exemplified in Fig. 6.5, oscillation level of one experiment session is defined as the mean stride-wise oscillation energy of the torque tracking error signal above $10 \mathrm{~Hz}$. The total oscillation energy of a signal $s(t)$ within one stance period is achieved by firstly high-pass filtering it at $10 \mathrm{~Hz}$. The filtered signal, $x(t)$, is converted to frequency domain using Fast Fourier Transform. The resulting signal in frequency domain, $X(f)$, is used to construct the energy spectral density as $X(f)^{2} \cdot T_{S}^{2}$. The total energy of oscillation of signal $s(t)$ is then calculated as the integral of the energy spectral density. The level of oscillation of a signal in one experiment session is then achieved by averaging the stride-wise torque error oscillation energy.

\subsection{Results}

The resulting stabilized passive stiffness values are listed in Table 6.4. Although the reported spring stiffness values span a huge range (Table 6.3), the actual maximum value is only around three times the minimum due to the existence of the Bowden cable synthetic rope in series with the spring, which exhibits the property of a nonlinear 


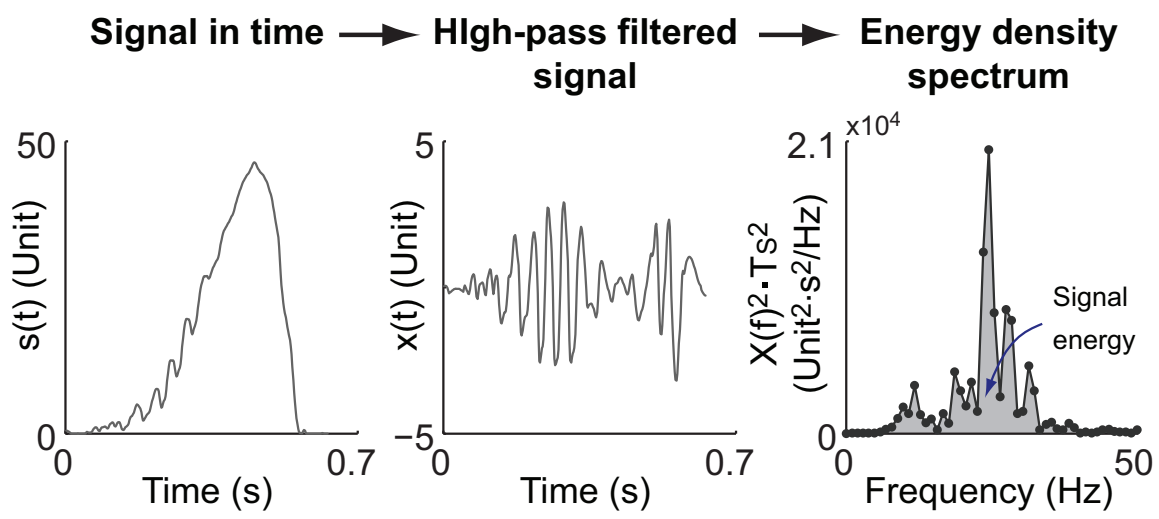

Figure 6.5: Demonstration of signal level of oscillation definition. A signal within one stance period in time domain, $s(t)$, is firstly high-pass filtered at $10 \mathrm{~Hz}$. The filtered signal, $x(t)$, is converted to frequency domain using Fast Fourier Transform. The resulting signal in frequency domain, $X(f)$, is used to construct the energy spectral density as $X(f)^{2} \cdot T_{s}^{2}$, in which $T_{s}$ is the sampling period of the signal. The total energy of oscillation of signal $s(t)$ is then the integral of the energy spectral density. The level of oscillation of a signal in one experiment session is then achieved by averaging that of every stride.

Table 6.4: List of measured stabilized passive stiffness values

\begin{tabular}{|c|c|c|c|c|c|c|}
\hline Passive Stiffness ID & S1 & S2 & S3 & S4 & S5 & S6 \\
\hline $\mathbf{K}_{\mathbf{t}}(\mathbf{N m} / \mathbf{d e g})$ & 1.9 & 2.8 & 3.7 & 4.7 & 5.6 & 5.9 \\
\hline
\end{tabular}

spring.

Over five hundred successful experiment trials, each identified by a unique combination of control gains, desired curve and passive stiffness, were conducted with different linear and piece-wise linear curves and used for data analysis.

Estimated optimal tracking errors, i.e., the RMS torque errors of the data sets with minimum errors, for linear curves are approximately linearly related to the absolute difference between desired and passive stiffness values as hypothesized by Hypothesis 1 and 2 (Fig. 6.6.A). It can be observed that torque errors show strong linear correlation with the absolute value of $K_{t}-K_{d e s}$ in cases of both individual desired curves and all curves combined. Minimum torque errors for all curves combined are linearly related 
to a translated absolute value of $K_{t}-K_{d e s}$, i.e,

$$
e_{\tau, o p t, R M S}=a \cdot\left\|K_{t}-K_{d e s}\right\|+b
$$

with a coefficient of determinant $R^{2}=0.839$ at a slope of $a=0.355$ for the absolute ones and $R^{2}=0.854$ at $a=0.869$ for the relative ones.

For piece-wise linear curves, the RMS torque errors of separate phases for data sets with minimum errors are also well correlated to their corresponding differences between the passive and desired stiffnesses (Fig. 6.6.B). The absolute and relative errors for all phases and curves combined are fitted with the translated absolute value of $K_{t}-$ $K_{d e s}$ with coefficients of determination $R^{2}=0.571$ and $R^{2}=0.497$ respectively. The slopes are $a=0.298$ and $a=0.691$. Note that for phases 1, 2 and 4, a fixed desired slopes exists in all steps of all data sets for the same desired curve. However, for phase 3 , since the peak dorsiflexion angle is different for each step of each data set, the desired slope for a trial with minimum errors is defined as the phase 3 slope in its average stride.

For the cases of both curve types, results (Fig. 6.6) agree with Corollary 2, and thus both Hypothesis 1 and 2, which serve as bases for it.

Control gains show interactions with desired and passive stiffnesses (Fig. 6.7). The proportional gains of the trials with minimum errors for all desired curves, which are the experimental estimates of optimal proportional gains, saw strong inversely proportional correlation with passive stiffness values $\left(R^{2} \geq 0.565\right)$. For each desired curve, data were fitted into a curve with the same format as Eq. (6.25), in which the same $\lambda$ values were asserted for all curves of the same type, i.e., linear or piece-wise linear. This result agrees with Hypothesis 3, which is based on Conjecture 1.

The $K_{p}-K_{t}$ coefficient, $\sigma$, as identified in Fig. 6.7, was also seen to be inversely 

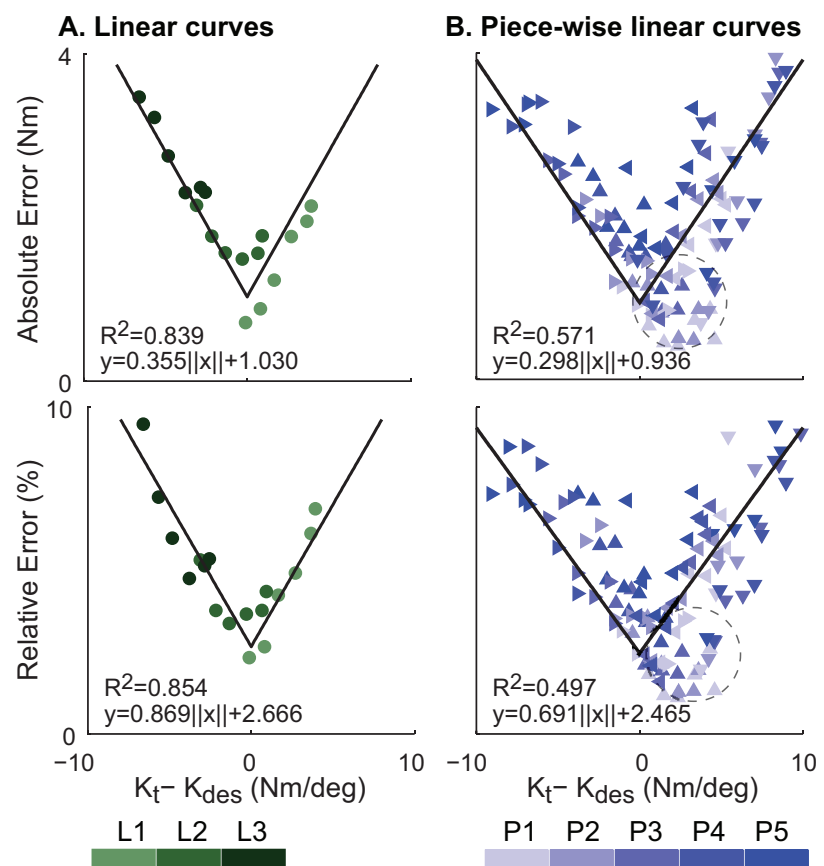

$\Delta$ Phase 1, 2, 3 and 4 in

$\nabla<$ piece-wise linear curves

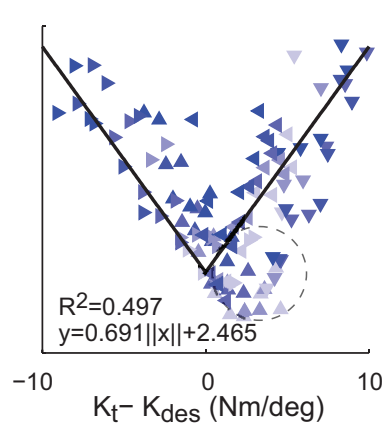

P1 P2 P3 $\quad$ P4 P5

Figure 6.6: Estimates of optimal torque tracking errors, i.e., those of the trials with minimum errors, for all combinations of passive stiffnesses and desired torque curves. (A) Torque errors for linear curves. (B) Torque errors for piece-wise linear curves with four phases presented separately. Row one presents the absolute errors. Row two presents the percentage of absolute errors relative to the peak desired torque of the corresponding experiment session. For both curve types, experimental estimates of minimum torque tracking errors show fairly strong linear correlation with the absolute values of the difference between the passive stiffness and the desired stiffness, $\left\|K_{t}-K_{d e s}\right\|$, which agrees with Hypothesis $\mathbf{1}$ and Hypothesis 2.

proportional to the desired stiffness (Fig. 6.8), which agrees with Hypothesis 4 based on Conjecture 1. Note that for each piece-wise linear curve, its effective desired stiffness is defined the mean of phase-wise desired stiffness values averaged over all the six best-performed data sets, one for each spring configuration.

\subsection{Discussion}

This chapter investigates the existence of an optimal passive stiffness that benefits torque tracking in lower-limb exoskeletons driven by series elastic actuator during walking. Based on theoretical analysis with simple transmission model, we hypoth- 

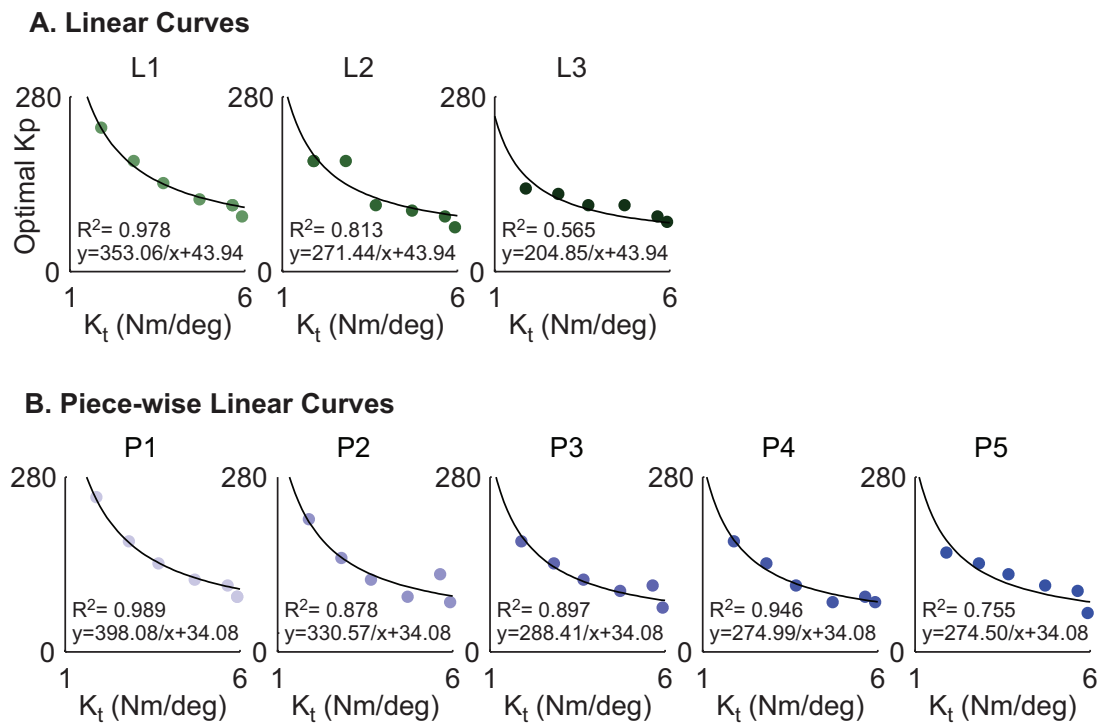

Figure 6.7: Values for the estimates of optimal proportional gain, $K_{p}$, i.e., those of the trials with minimum errors, of various passive stiffness configurations show fairly strong inverse proportional correlation with the respective passive stiffness values for all desired curves $\left(R^{2} \geq\right.$ 0.565), which agrees with Hypothesis 3.

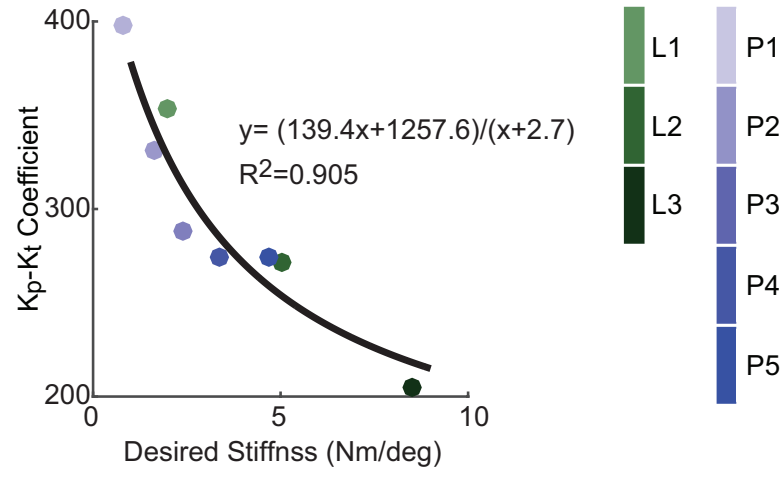

Figure 6.8: Values of gain-stiffness inverse correlation coefficient, $\sigma$, which were achieved by curve fitting in Fig. 6.7, are inversely proportional to the desired stiffness values of various curves. This agrees with Hypothesis 4. The effective desired stiffness of a piece-wise linear curve is defined the mean of desired stiffness values across four phases averaged over all the six best-performed data sets, one for each passive stiffness configuration

esized that to achieve best torque tracking during walking in exoskeletons, the actuator should be designed such that its passive stiffness, defined as the slope of device torqueangle relationship with motor position fixed, matches the desired quasi-stiffness. We also hypothesized a fixed maximum torque error changing rate that leads to the inverse proportional correlations between optimal proportional gains and passive stiffness values for a fixed desired curve. These hypotheses were highly agreed by a large amount 
of walking experiment data.

Although only a simplified model of the transmission sub-system was considered, torque tracking results in Fig. 6.6 for linear curves highly agrees with Hypothesis 1 $\& 2$. However, the phase-wise errors for piece-wise linear curves show slightly less agreement with the hypothesis. One reason is that the control gains were optimized based on full-step instead of phase-wise performance. According to the interactions between optimal proportional gains, desired stiffness and passive stiffness presented in Fig. 6.7, for the same passive stiffness configuration, a larger desired stiffness results in a smaller optimal proportional gain. However, the level of oscillations and step-wise root-mean-squared torque errors are collectively determined by tracking performance of all four phases. Therefore, the optimal proportional gain for a piece-wise linear curve is expected to be higher than the optimal gain for the phase with largest desired stiffness and lower than the one with smallest. This means that the phase-wise torque errors in piece-wise linear curves are noisier than those of linear curves. Another issue was that for some phases, for example phase 1 of $\mathrm{P} 1, \mathrm{P} 2$ and $\mathrm{P} 3$, the desired torques were very low. Since the Bowden cable rope was still slacking at the beginning of stance, the effective passive stiffness values were actually a lot smaller than the stabilized values we used in data analysis. Therefore, many data points as circled in Fig. 6.6.B should be shifted to the left, which will improve the fitting. We also attempted to evaluate the effective difference in desired and passive stiffness, $K_{t}-K_{d e s}$, of piece-wise linear curves for full steps and present torque errors in a way similar to the linear curves in Fig. 6.6.A. One way we tried is to generate the effective desired stiffness of piecewise linear curves by linearly fitting the average stride and use it to then calculate $K_{t}-K_{d e s}$. Another method tried is to calculate the difference as the area between desired stiffness versus torque curve and passive stiffness versus torque curve. For both cases, the relationships between torque errors and effective stiffness differences showed significantly less agreement with Eq. (6.23) than Fig. 6.6.B. This suggest that when Hypothesis 1 and 2 are used in guidance to choose passive stiffness, the concerning 
desired stiffness value $K_{t}$ should be the instantaneous values instead of a collective determined values.

Meanwhile, there are other factors that add noise and complexions to the data, which causes imperfection in curve fitting and non-zero torque errors at $K_{t}=K_{d e s}$ as shown in Fig. 6.6. First factor is the experimental method used. The optimal performance of each desired and passive stiffness combination were achieved by gradually increasing proportional and damping injection gains until perceptible oscillations happen with maximum damping gains. There are multiple noise sources cased by this test scheme. The most obvious one is the testing of discrete gain values, which results in the fact that the gain values of the best-performed experiment session are mostly not the optimal gains but actually values close to them. Second, increase of control gains stop when the oscillations become noticeable for the subject, which makes the stopping criteria subjective. Although the same subject was use throughout all experiments, adaptation and subject physical condition both affect the subject's judgment of when discomfort starts, which potentially leads to higher gains tested when the subject has higher tolerance. In some cases, increase of gains stop before the torque errors hit minimum due to inability of human to tolerate oscillations, which affects the estimation of minimum torque errors and optimal control gains. Besides subjectivity of testing, actual changes in system dynamics also causes noises in data. These changes include subject physical condition across experiment sessions, human body instant mechanical properties changes due to muscle tensioning, gait variations and movements in human-exoskeleton interface. Another reason that led to imperfection in the alignment between theory and experiment results is the employment of a highly simplified system partial model. Due to the presence of nonlinear, uncertain, highly complex and changing dynamics, a lot of system features were not captured in the theoretical hypothesis. One complication that contributed was the nonlinear property of the system passive stiffness due to the slow stretching property of the Vectran ${ }^{\circledR}$ cable as demonstrated by Fig. 6.3. Due to the unstructured changes of passive stiffness between different loads and trials, only one stabilized value was used 
for each passive stiffness configuration. Another feature that causes complication into system dynamics but was not accounted for in theoretical analysis was the highly nonlinear, complex and changing frictions in Bowden cable. We also made the assumption of immediate perfect motor position tracking, which is not true in practical cases due to the limitation of motor velocity. This greatly contributed to the fact that when the passive stiffness matches desired stiffness, i.e., $K_{t}=K_{d e s}$, torque errors is above zero under optimal control conditions.

Regardless of the various approximations made in various hypotheses, the results presented Fig. 6.6, 6.7 and 6.8 support them with fairly strong correlations. The conjecture of a fixed bandwidth and thus a maximum torque error tracking rate, $\dot{e} \tau$, max, as a limit for proportional gain increase suggests a potential way of systematic gain tuning when desired or passive stiffness is changed for the same subject. Since the dependence of this maximum error changing rate on full system dynamics, it is expected it is subject-dependent for the same motor system. However, since the primary goal of this study is the identification of the optimal passive stiffness for a desired stiffness, only one subject is used. Therefore, how the maximum tolerable torque changing rate varies among subjects remains a direction of future work.

\subsection{Conclusions}

This study hypothesizes and confirms by experiments that the optimal passive stiffness for lower-limb exoskeleton torque tracking corresponding to the desired quasi-stiffness, which is the slope of desired torque-angle relationship. The best torque tracking performance is shown to be linearly related to the difference between the desired and passive stiffnesses. This study also hypothesizes a potential maximum torque error tracking rate and therefore control bandwidth that prevents the proportional feedback gain from increasing infinitely. This was also supported by experimental data. These results provide 
guidance for passive stiffness selection of lower-limb exoskeletons and other walking related robots for a fixed desired stiffness. They also provide guidance for optimal gain tuning in case of changing passive or desired stiffness.

\subsection{Acknowledgment}

This material is based upon work supported by the National Science Foundation under Grant No. IIS-1355716. 


\section{Chapter 7}

\section{High Level Control Online \\ Optimization}

\subsection{Introduction}

Despite of various efforts made to improve the controller performance on ankle exoskeletons and prosthesis, it remains unclear what kind of control objectives, i.e., highlevel controller, are best accepted by human body. Due to the complexity of human physical and chemical system, human responses change from person to person and from time to time. Therefore, for each particular subject at a certain experiment day, an automated calibration and tuning process which determines the best suited high level controller will be beneficial to training and experimental studies. This defines an online optimization process that maximize human benefit of certain definition by choosing the best high level controller elements.

Real-time human metabolic energy consumption is one direct indication of human reactions in experiments, in which maximized human benefit is represented by mini- 
mized energy consumption. Previous research has been done for real-time human energetic cost optimization by changing step frequency using gradient based methods [167]. However, none of the methods previous demonstrated is suitable for multivariate parameter optimization and high level control conditions that takes time to be enforced. Evaluation time will blow up fast. This study therefore extends previous concepts to the parameterization of multivariate lower-limb exoskeleton high level controller for minimized metabolic energy cost. It aims to develop a framework for rapidly-converging on-line optimization of assistive robots like exoskeletons, orthoses and prostheses.

Classical gradient-based methods such as gradient descent method [168] have been widely used in optimization due to simplicity. However, the need to calculate local gradients and sensitivity to noise makes it less feasible for our problem, in which fairly long time is needed for evaluation, and a high noise-to-signal ratio is present.

Direct search methods, which are gradient-free, seem more promising for our problem. Covariance Matrix Adaption-Evolutionary Strategies (CMA-ES) [38] are stochastic, derivative-free, and they work for non-linear and non-convex continuous problems, which are beneficial to our issue. The generation size does not increase severely with increasing parameter size. However, there are still relatively large number of evaluations needed to make one decision. Surrogate-based methods [169] can make a decision after each evaluation after the initial process. However, the number of initial evaluations blows up rapidly as the parameter size increases. Nelder-Mead method, i.e., downhill simplex method [170] makes a decision with each new evaluation and has a small initial evaluation size.

This study develops an online optimization system of multivariate high level controllers of assistive robots using metabolic measurements. The system is tested on an ankle exoskeleton with a four-parameter high level controller that determines desired torque based time. The effectiveness of the system and various algorithms were tested 
in walking experiments and verified through validation tests. The resulting protocol is expected to work in various human-robot interactive systems, conduction of which at the begining of long experimental, therapeutic or assistive sessions will help to improve human experience, experimental accuracy, training efficiency or assistive performance.

\subsection{Methods}

Online optimization of multi-parameter high-level controllers of ankle exoskeletons from real-time metabolic energy cost were developed, implemented on a powered ankle exoskeleton. A four-parameter controller was tested in experiments to demonstrate the functionality of the system. Online optimization was accomplished through two algorithms, Nealder-Mead method and Covariance Matrix Adaptation Evolution Strategy (CMA-ES). Each sample point includes an input of a four-dimensional high level controller parameter set and an output that is the estimate of the metabolic rate from real-time respiratory measurements within two minutes of treadmill working while the subject wears the ankle exoskeleton on the right leg under the input condition. For both methods, thirty two conditions generated by the algorithm were evaluated during online optimization. Both optimized conditions were then tested in comparison with two control conditions in validation process to verify the effectiveness of the online optimization process using the two algorithms.

\subsubsection{Exoskeleton Testbed}

The online optimization system was implemented on a tethered ankle exoskeleton comprised of an off-board real-time control module and geared electric motor, a unidirectional Bowden cable transmission with a series leaf spring, and an exoskeleton frame that interfaced with the human foot and shank (Fig. 7.1). 


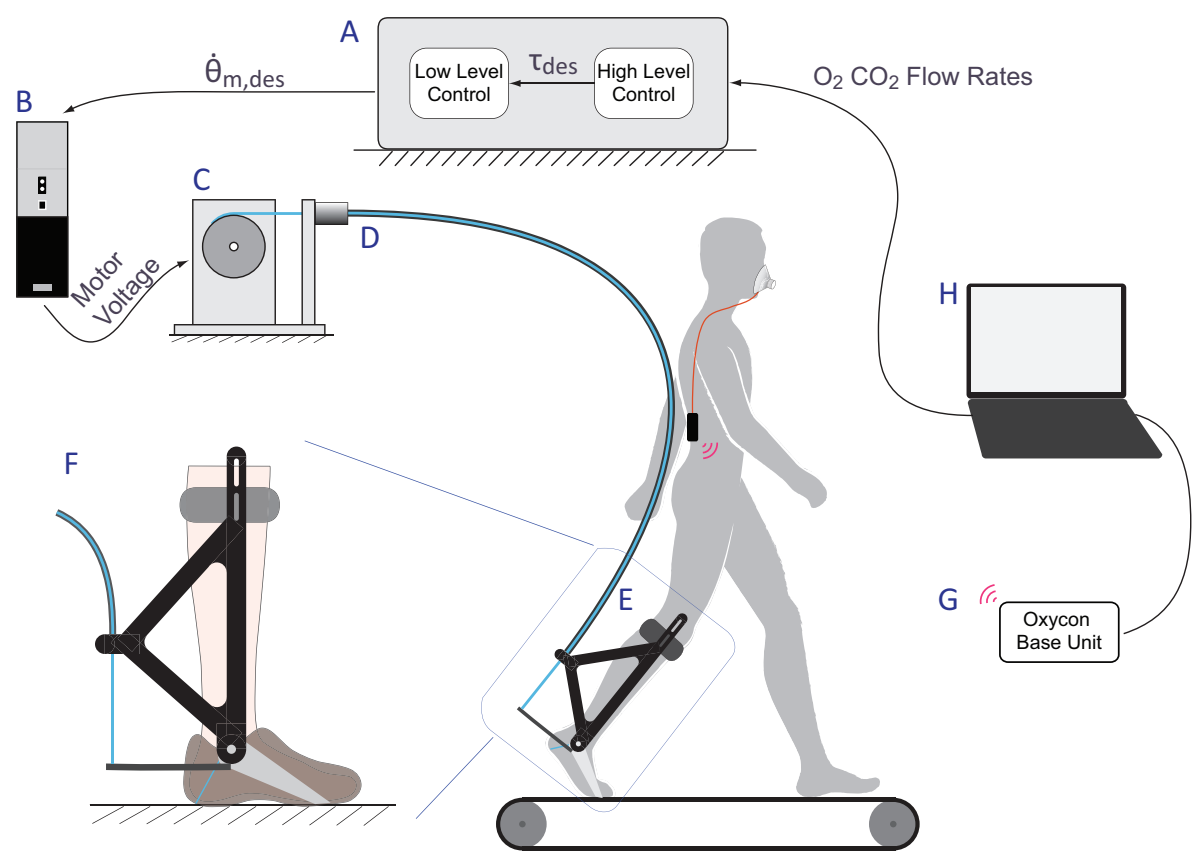

Figure 7.1: The ankle exoskeleton testbed system used in experiments of this study. A) A real-time high-speed controller DS1103 (DSpace Inc.) that reads sensory readings, generates control objectives using high level controllers and generates motor velocity commands using low level controllers. B) Motor drive. C) High speed motor. D) Bowden cable transmission. E,F) The ankle exoskeleton used. G) Base unit of Oxycon mobile that receives and processes flow rates measurements. H) Computer used to display, screen read and transmit respiratory measurements. 
A dedicated real-time control system (ACE1103, dSPACE Inc.) sample sensors at $5000 \mathrm{~Hz}$, filter sensor data at $200 \mathrm{~Hz}$, and generate control commands at $500 \mathrm{~Hz}$. The motor unit was composed of a low-inertia $1.6 \mathrm{~kW}$ AC servo motor and a 5:1 planetary gear, with input voltage regulated by a motor driver running in velocity control mode (BSM90N-175AD, GBSM90-MRP120-5 and MFE460A010B, Baldor Electric Co.). A digital optical encoder (E5, US Digital Corp.) measured motor position.

The exoskeleton frame applied forces on the front of the human shank below the knee, beneath the heel, and beneath the toe, so as to generate an ankle plantarflexion torque in proportion to transmission force. Torque was measured using a load cell (LC201 Series; OMEGA Engineering), with 1000 Hz signal conditioning (CSG110, Futek Inc.). Joint angle was measured using a digital optical encoder (E8, US Digital Corp.).

A flexible uni-directional Bowden cable transmitted forces from the motor to the exoskeleton frame while minimally restricting leg motions. The cable was composed of a coiled-steel outer conduit (415310-00, Lexco Cable Mfg.) and a 0.003 m diameter Vectran ${ }^{\circledR}$ inner rope, and was $2 \mathrm{~m}$ in length. A series spring was attached at the end of the rope to provide increased compliance. This spring is switched in tests to investigate the effects of changing passive stiffness on torque tracking in the device.

Human metabolic rate was indirectly measured through breath-by-breath respiratory measurements. The flow rates of $\mathrm{O}_{2}$ and $\mathrm{CO}_{2}$ were measured by a wireless, portable cardiopulmonary mobile sensory system (Oxycon ${ }^{\mathrm{TM}}$ Mobile, Carefusion Corp.). It is composed of a volume sensor connected to a local preliminary analyzer attached to human back, which also serves as a wireless transmitter and continuously sends data to a stationary volume and gas analyzer. The calibrated and processed measurements was transmitted through wired connection and displayed on a local computer. The real time values of flow rates were then automatically read from the computer screen through 
image-to-text recognition and sent to the controller through serial communication using Sikuli scripts.

\subsubsection{Parameters to Be Optimized: High Level Controller}

The goal of this project is to optimize multiple parameter high level controllers in human-robot interactions, aiming to minimize human metabolic cost. The online optimization system was tested for a four-parameter high level torque controller of ankle exoskeletons as shown in Fig. 7.2. It is a desired torque curve that is based on the

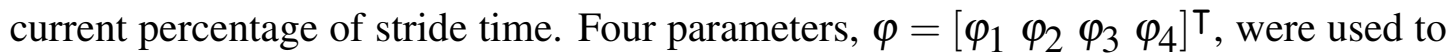
define the three anchor points. $\varphi_{1}$ represents peak desired torque. $\varphi_{2}$ is the percentage of stride time since heel stride when peak desired torque is happening. $\varphi_{3}$ is the percentage of stride time between $A_{1}$ and $A_{2}$, or 'rise time' as called in this study. $\varphi_{4}$ denotes the percentage of stride time between $A_{2}$ and $A_{3}$, or 'drop time'. In between $\left(\varphi_{2}-\varphi_{3}\right) \%$ and $\left(\varphi_{2}+\varphi_{4}\right) \%$ of stride time, the desired torque is a cubic spline that passes anchor points $A_{1}=\left[\varphi_{2}-\varphi_{3}, 2\right], A_{2}=\left[\varphi_{2}, \varphi_{1}\right]$ and $A_{3}=\left[\varphi_{2}+\varphi_{4}, 2\right]$. At heel strike, i.e. $0 \%$ of the stride period, and $65 \%$ of stride time which is roughly when stance ends, desired torque values are set as zeros. Before $A_{1}$ and after $A 3$, the desired torque is then linearly interpolated.

Literature and prior experiments suggested that the peak time $\varphi_{2}$ and drop time $\varphi_{4}$ have relatively smaller ranges that are comfortable for human compared to the other two parameters. Therefore, in order to make all parameters comparable and to improve the performance of optimization process, the original parameter $\varphi=\left[\begin{array}{lll}\varphi_{1} & \varphi_{2} & \varphi_{3}\end{array} \varphi_{4}\right]^{T}$ is re-parameterized into $\psi=\left[\begin{array}{llll}\psi_{1} & \psi_{2} & \psi_{3} & \psi_{4}\end{array}\right]^{T}$, in which $\psi_{1}=\varphi_{1}, \psi_{2}=2 \varphi_{2}, \psi_{3}=\varphi_{3}$ and $\psi_{4}=2 \varphi_{4}$. 


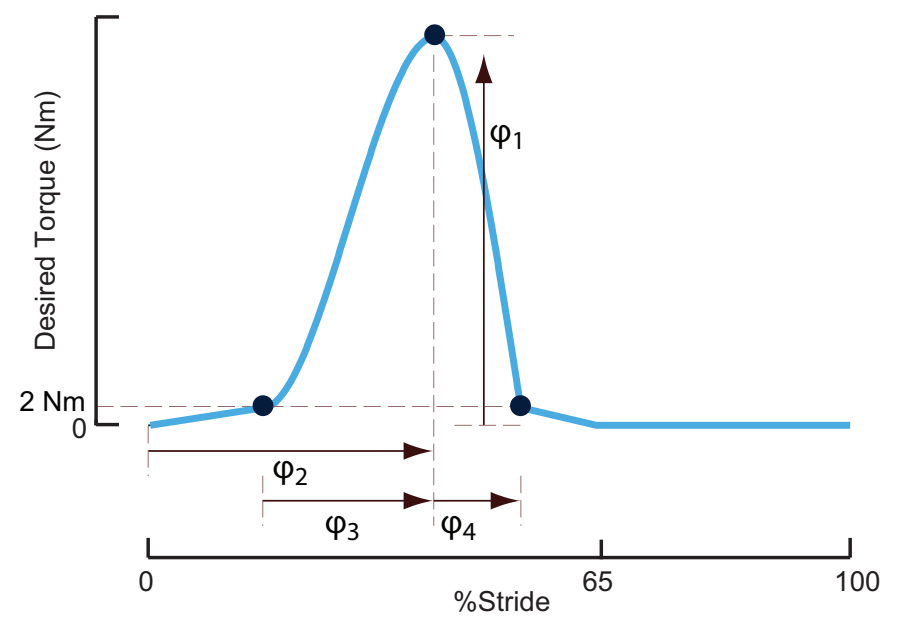

Figure 7.2: The high-level controller used in experiments of this study: a desired torque curve defined by the 4-D design vector $\varphi=\left[\begin{array}{lll}\varphi_{1} & \varphi_{2} & \varphi_{3}\end{array} \varphi_{4}\right]^{\top}$, elements of which represent peak desired torque, \%stride time of peak torque happening, \%stride time of the peak torque rise time, and $\%$ stride time of the peak torque drop time, or 'drop time' as called. In between $\left(\varphi_{2}-\varphi_{3}\right) \%$ and $\left(\varphi_{2}+\varphi_{4}\right) \%$ of stride time, the desired torque is a cubic spline that passes anchor points $\left[\varphi_{2}-\varphi_{3}, 2\right],\left[\varphi_{2}, \varphi_{1}\right]$ and $\left[\varphi_{2}+\varphi_{4}, 2\right]$. Within $\left[0 \varphi_{2}-\varphi_{3}\right] \%$ and $\left[0 \varphi_{2}+\varphi_{3} 0\right] \%$ of stride time, the desired torque is linearly interpolated between 0 and $2 \mathrm{Nm}$.

\subsubsection{Low Level Torque Controller: Proportional Control + Damp- ing Injection + Iterative Learning}

To realize the different high level control conditions, the combination of model-free, integral-action-free feedback control and iterative learning $[60,61]$ was used to generate motor velocity command for torque tracking. The controller is expressed as

$$
\begin{aligned}
\dot{\theta}_{p, d e s}(i, n)= & \underbrace{-K_{p} \cdot e_{\tau}(i, n)}_{\text {Proportional Control }}+\underbrace{-K_{d} \cdot \dot{\theta}_{p}(i, n)}_{\text {Damping Injection }} \\
& +\underbrace{\dot{\theta}_{p, \text { des }}^{L R N}(i+D, n)}_{\text {Iterative Learning }} \\
\dot{\theta}_{p, d e s}^{L R N}(i, n+1)= & \beta \dot{\theta}_{p, \text { des }}^{L R N}(i, n)-K_{l} \cdot e_{\tau}(i, n) \\
\dot{\theta}_{m, d e s}= & N \dot{\theta}_{p, \text { des }}
\end{aligned}
$$


in which $n$ denotes the current step number and $i$ the index count within the current step. $\dot{\theta}_{p, d e s}$ is the desired motor pulley velocity and $\dot{\theta}_{m, d e s}$ is the motor velocity to be commanded. $N$ denotes the gear ratio. $e_{\tau}=\tau-\tau_{d e s}$ is the torque error. $K_{p}, K_{d}$ and $K_{l}$ are the proportional, damping and iterative learning gains. $\dot{\theta}_{p, d e s}^{L R N}$ is the motor velocity command generated from iterative learning. $\beta$ is a forgetting factor used to improve the stability of tracking. $D$ denotes the index count iterative learning command looks into the future.

\subsubsection{Estimation of Metabolic Rates from Respiratory Measure- ments}

The flow rates of $\mathrm{O}_{2}$ and $\mathrm{Co}_{2}$ are converted to raw metabolic rates using a variation of Brockway's equation [173],

$$
y=\dot{E}(\text { Watts })=\frac{16.477 \dot{V} O_{2}+4.484 \dot{V} C O_{2}}{60}\left(\frac{\mathrm{ml}}{\min }\right)
$$

where $\dot{V} O_{2}$ and $\dot{V} C O_{2}$ are the raw measurements of human respiratory $\mathrm{O}_{2}$ and $\mathrm{CO}_{2}$ flow rates respectively, and $\dot{E}$, or $y$ as used in later equations, is the corresponding raw metabolic rate measurement of human body.

Due to the complicated chemical dynamics of human body and high breath-to-breath variation of respiration, the actual human muscle energy consumption is not linearly corrected to the respiratory measurements. Instead, its governing dynamics is shown to be estimable by a first order linear system with a time constant of $42 \pm 12 \mathrm{~s}$ [171]. To better reflect the metabolic energy consumption during walking experiments, the raw respiratory measurements will be converted into better estimates of actual instantaneous energetic cost by reversing the dynamics as follows.

For a constant high level control condition that the exoskeleton is applying to human 
body, a constant actual metabolic cost $\mathrm{R}$ is expected from human body. The measurable metabolic cost $y$ is expected to be related to $\mathrm{R}$ by

$$
\dot{y}(t)=\frac{1}{\delta}(u(t)-y(t))
$$

in which the input $u(t)$ is always $\mathrm{R}$ and $\delta$ denotes the time constant. This continuous dynamics can be discretized into

$$
y(i+1)=\frac{1-d t(i)}{\delta} y(i)+\frac{d t(i)}{\delta} \mathrm{R}
$$

in which $d t(i)=t(i+1)-t(i)$ is the time difference between samples $y(i)$ and $y(i+$ 1). Assume this condition results in a sequence of raw metabolic rate measurements sequence $y_{m}=\left[y_{m}(1) y_{m}(2) \ldots y_{m}(m)\right]$ with time stamps $t=[t(1) t(2) \ldots t(m)]$.

$$
A \cdot\left[\begin{array}{c}
y(1) \\
\mathrm{R}
\end{array}\right]=\left[\begin{array}{c}
y_{m}(1) \\
y_{m}(2) \\
\vdots \\
y_{m}(m)
\end{array}\right]
$$

in which matrix $A \in \mathbb{R}^{m x 2}$ is constructed as below.

$$
\begin{aligned}
& A(1,1)=\frac{1-d t(1)}{\delta} \\
& A(1,2)=\frac{d t(i)}{\delta}
\end{aligned}
$$

and if $i \in[1,2, \cdots, m]$,

$$
\begin{aligned}
& A(i, 1)=\frac{1-d t(i-1)}{\delta} \cdot A(i-1,1) \\
& A(i, 2)=\frac{1-d t(i-1)}{\delta} \cdot A(i-1,2)+\frac{d t(i-1)}{\delta}
\end{aligned}
$$


according to Eq. (7.4). Then the initial metabolic rate output $y(1)$ and the constant actual metabolic cost $\mathrm{R}$ can be estimated as

$$
\left[\begin{array}{c}
\hat{y}(1) \\
\hat{\mathrm{R}}
\end{array}\right]=A^{+} y
$$

This estimate of metabolic rate $\hat{\mathrm{R}}$ is then used as the output cost in online optimization. An example is shown in Fig. 7.3, in which the raw metabolic rate measurements is estimated as the output of a first order low pass filter dynamics with time constant of $42 \mathrm{~s}$ and a step input. The identified constant step input servers as the estimate of the metabolic cost of this period.

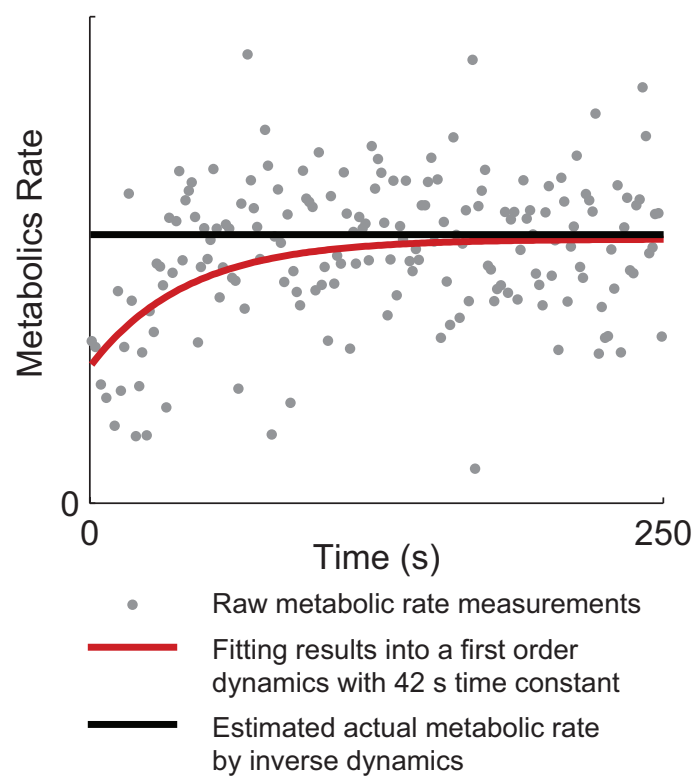

Figure 7.3: Estimation of a single actual metabolic rate [171] from multiple raw measurements within a peroid by inversing a first order dynamics with a time constant of $42 \mathrm{~s}$.

\subsubsection{Optimization Algorithms}

Based on literature research and simulations, two algorithms that were selected in online optimization process: memoryless CMA-ES [38] and Nelder-Mead method [170]. CMA-ES is a stochastic optimization algorithm which causes it to be insensitive to the 
high noises of metabolic measurements. When it is used in a memoryless way, i.e., each new decision is made based on a newly generated set of randomly generated sample conditions called a generation, being trapped to a local minum is avoided. NelderMead is simple and straight forward, which produces a new estimate of the optimum for each evaluation after $N$ ones, in which $N$ is the parameter size. Neither of them needs evaluation of local gradient values, construction of which heavily increases evaluation numbers that we cannot afford due to the long time needed to make one evaluation ( 2 minutes). However, memoryless CMA-ES needs large numbers of evaluations every generation to make one update to the optimum while Nelder-Mead is more sensitive to noise. Both algorithms are expected to scale well,i.e., the total number of evaluations needed during optimization increases relatively slowly as parameter number increases.

\subsubsection{Experimental Methods}

The on-line optimization on high-level torque controller was tested with one healthy subject $(\mathrm{N}=1,32$ years, $55 \mathrm{~kg}, 164.5 \mathrm{~cm})$ walking on a treadmill with a constant speed of $1.25 \mathrm{~m} / \mathrm{s}$ at self-paced step frequency while wearing the tethered ankle exoskeleton on the right leg. All experimental protocols were approved by the Carnegie Mellon University Institutional Review Board.

The initial high level control condition was randomly sampled at a mean of $\psi=$ [30 9025 20] with a covariance of $\operatorname{diag}(100100100$ 100) which was comfortable and reasonable based on pilot sampling of the parameter space. Low level torque controller parameters in Eq. (7.1) were set as $K_{p}=3 \mathrm{deg} / \mathrm{s} / \mathrm{Nm}, K_{d}=0.02, K_{l}=0.8 \mathrm{deg} / \mathrm{s} / \mathrm{Nm}$, $\beta=0.995$ and $D=7$ which meant $14 \mathrm{~ms}$ of prediction from iterative learning, according to initial sweep and tuning.

Pilot tests of six minutes walking with fixed high level conditions demonstrated the high variations in optimal time constant for metabolic cost estimation, i.e., $T$ in 
Eqs. (7.3) (7.4) and (7.5), while the estimate varies less 5\%. Therefore, $42 \mathrm{~s}$ was used for all conditions in optimization. Using this time constant, a length of 120 seconds was used for each condition during online optimization, which was demonstrated to produce estimation errors of less than $15 \%$ than that of six minutes of data.

For CMA-ES, the generation size was set as eight. In Nelder-Mead, the reflection, expansion, contraction and shrink coefficients were $1,2,0.5$ and 0.5 respectively. For both methods, thirty-two conditions (sixty-four minutes) were tested during online optimization, which meant four generations for CMA-ES. The optimized condition for CMA-ES (Condition A) is defined as the mean of the fifth generation and that of Nelder-Mead (Condition B) is the mean of the last five conditions.

For CMA-ES, with guide generation size [38] of

$$
\lambda=4+\text { floor }(3 \ln (N))
$$

in which $N$ is the parameter size, the total evaluation time in optimization process is

$$
T_{\text {opt }}=4 \times \lambda \times \Delta T
$$

in which $\Delta T$ stands for the time length of each evaluation. In our protocol, $\Delta T=2 \mathrm{mins}$, therefore,

$$
T_{\text {opt }}(N)=32+8 \times \text { floor }(3 \ln (N))(\text { mins })
$$

which suggests an average evaluation time in minutes per parameter of

$$
\frac{T_{o p t}}{N}(N)=\frac{32+8 \times \text { floor }(3 \ln (N))}{N}
$$

as illustrated in Fig. 7.4. It can be seen that as parameter size increases, the cost of evaluation exponentially. 


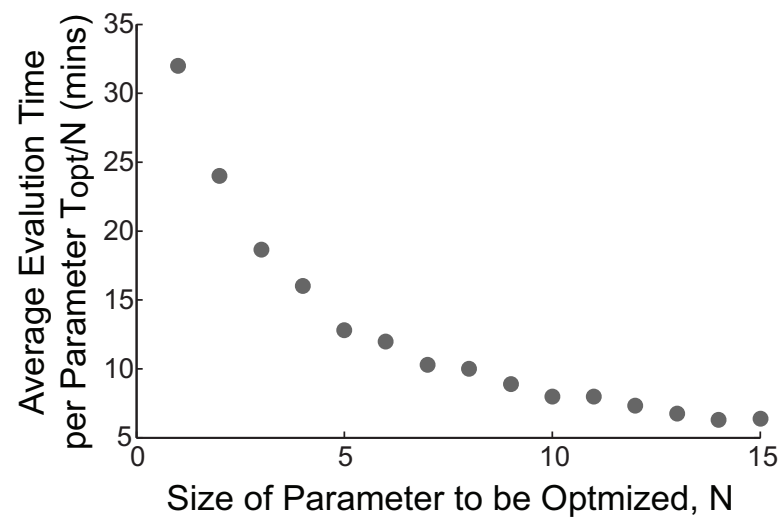

Figure 7.4: Total CMA-ES optimization evaluation time per parameter with four generations of evaluations and evaluation sample length of 2 minutes.

For validation, the optimized conditions from both algorithms were tested in comparison with two control conditions: the zero impedance condition (Condition $\mathrm{C} 1)$ in which the exoskeleton follows human ankle while applying no torques and a previous hand tuned condition (Condition C2) which was a hand tuned time based torque profile which was shown to have a $17 \%$ benefit of metabolic cost from zero impedance in a previous study [172]. To eliminate the effects of potential metabolic cost drift in validation, the four conditions are tested in the sequence of $\mathrm{C} 2 \rightarrow \mathrm{A} \rightarrow \mathrm{B} \rightarrow \mathrm{C} 1 \rightarrow \mathrm{B} \rightarrow \mathrm{A} \rightarrow \mathrm{C} 2$. All conditions except zero impedance were tested in two sessions. Each validation experiment session takes six minutes of walking on the treadmill with the desired condition, and in between sessions, there were three minutes of resting period in which the subject stood still. Each validation session produced one estimation of metabolic rate using the inverse dynamics method (7.8). The average of estimated metabolic costs across sessions with the same conditions were used to indicate the metabolic costs.

\subsection{Results}

Online optimization processes using both CMA-ES and Nelder-Mead, each of which includes full evaluation of thirty two automatically generated conditions, are presented in Fig. 7.5 and 7.6 respectively. CMA-ES optimization process showed drop of mean 
metabolic cost each generation and fair convergence during the last. Nelder-Mead optimization process showed convergence of parameters and metabolic costs faster, but the drop in metabolic cost compared to the initial conditions was less severe.

Results of validation process comparing the metabolic costs of the two optimized conditions, zero impedance condition and a previous identified energy-efficient condition is shown in Fig. 7.7. Both inward (conditions 1-4) and outward (conditions 4-7) comparison cycles showed the same trends of metabolic cost as Zero Impedance > Hand Tuned $>$ Nelder-Mead $>$ CMA-ES. Averaging the results of sessions with the same conditions, a drop of $26.9 \%$ from zero impedance condition was observed for CMA-ES condition. The drops were $20.5 \%$ and $16.4 \%$ for Nelder-Mead and hand tuned conditions respectively.

\subsection{Discussion}

This study develops a system that optimizes the high level controllers of human-robot interactive systems online using CMA-ES and Nelder-Mead algorithms from real-time human respiratory measurements. The system was implemented on an ankle exoskeleton testbed, with a high level controller defined as a four-parameter time based desired torque curve. With thirty-two conditions evaluated in sixty-four minutes during optimization, the optimized conditions of both algorithms metabolic cost drop from zero impedance and an energy-efficient control condition identified in one prior study. CMA-ES showed better performance than Nelder-Mead with a metabolic cost drop of $26.7 \%$ and was less sensitive to noise and is therefore preferred. Nelder-Mead showed faster convergence of parameter but has the risk of being trapped in local minimum or a small evaluation from noise. These results demonstrated the effectiveness of the online optimization system using metabolic measurements and identifies the preferred algorithm of sample generation as CMA-ES. 
A. CMAES online optimization condition parameter values and metabolic measurements
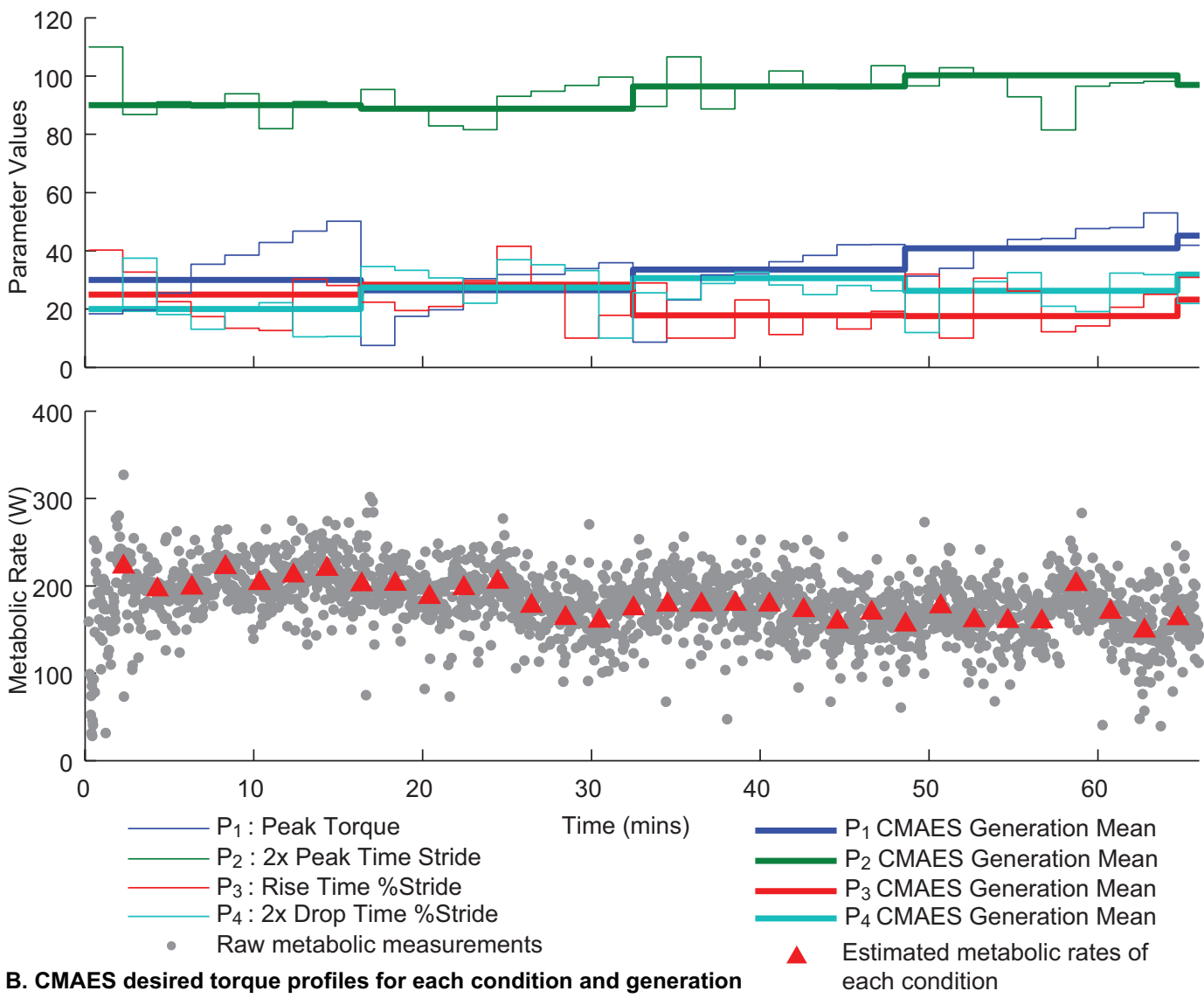

B. CMAES desired torque profiles for each condition and generation each condition
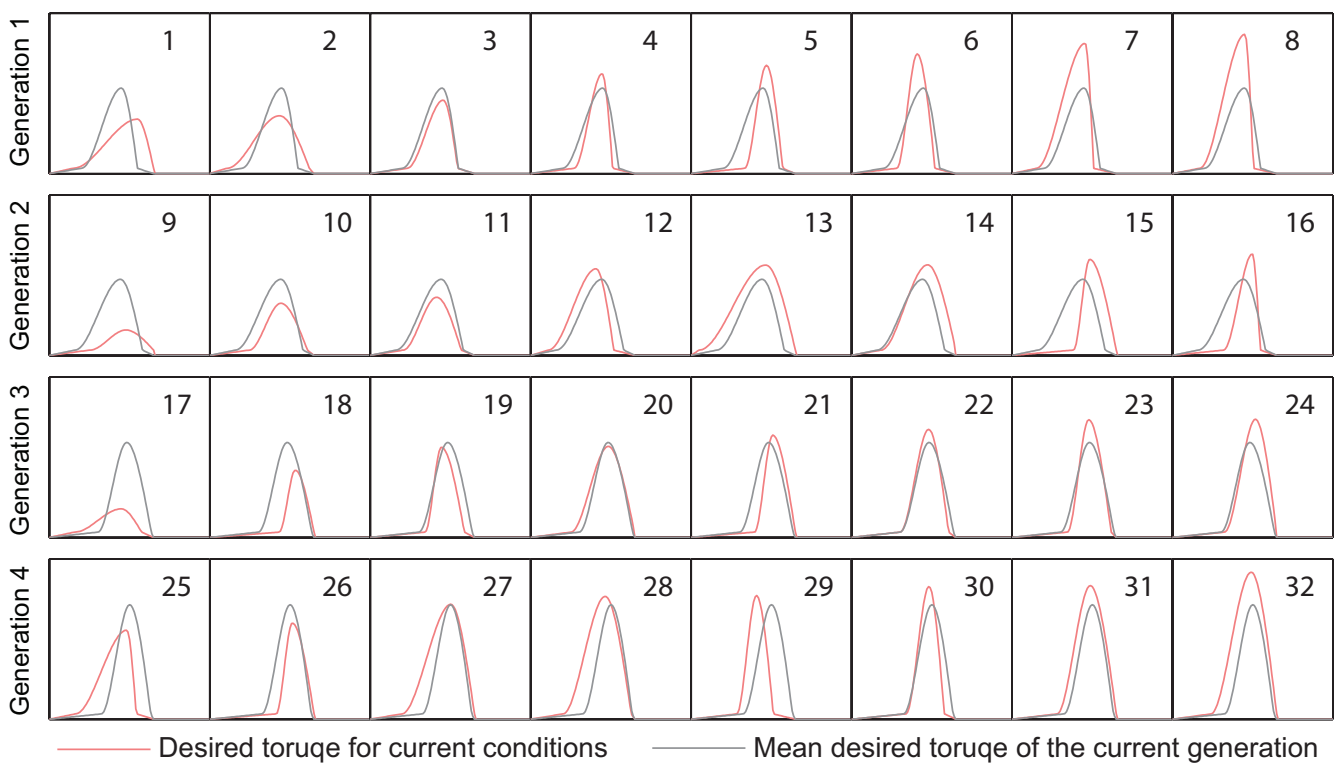

Figure 7.5: Online optimization process using CMA-ES. Four generations, thirty two conditions, sixty-four minutes of walking were automatically generated and evaluated. The optimized high level condition is defined by the parameter means of the fifth generation which were produced at the end of the fourth one. 
A. Nelder-Mead online optimization condition parameter values and metabolic measurements
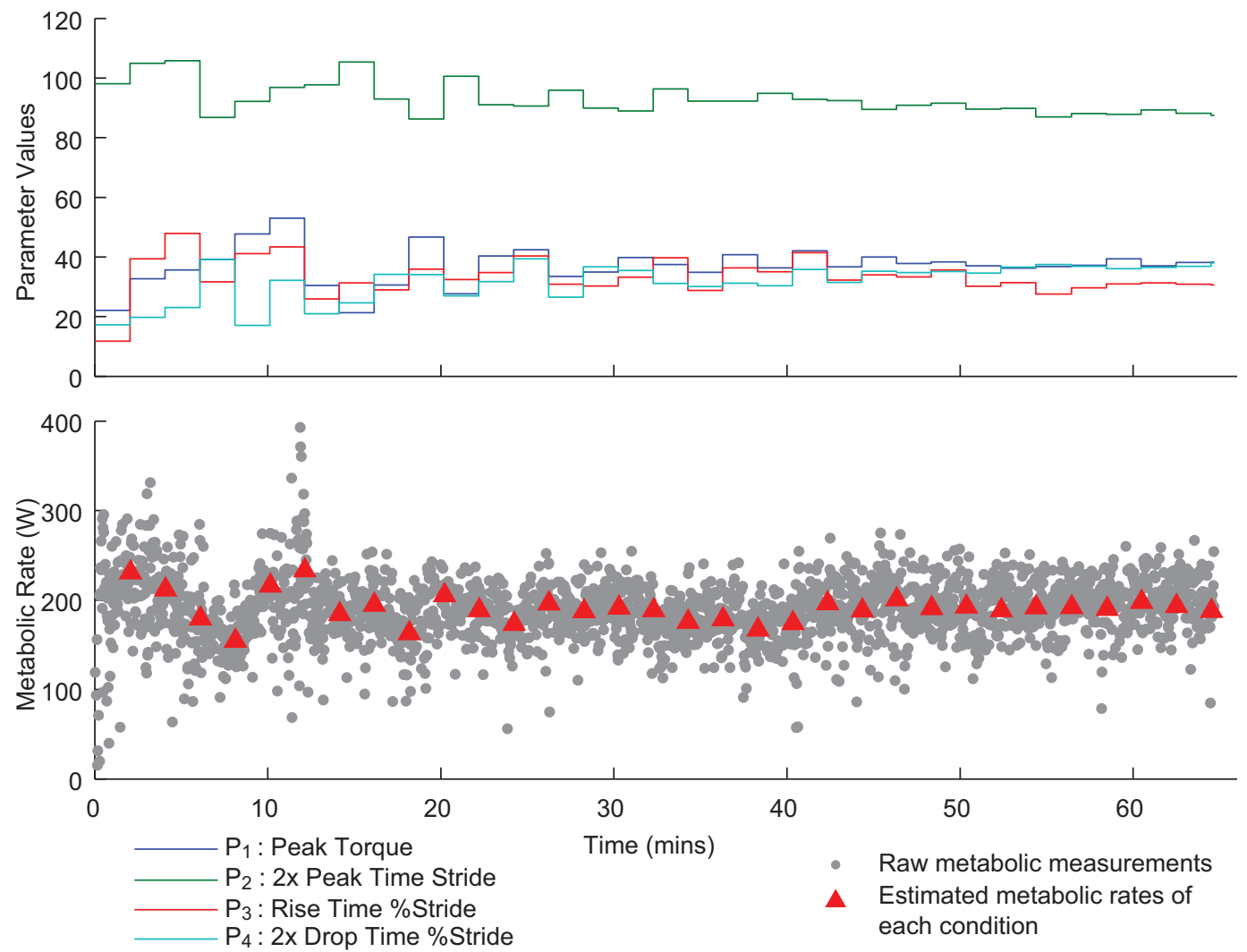

B. Nelder-Mead desired torque profiles for each condition
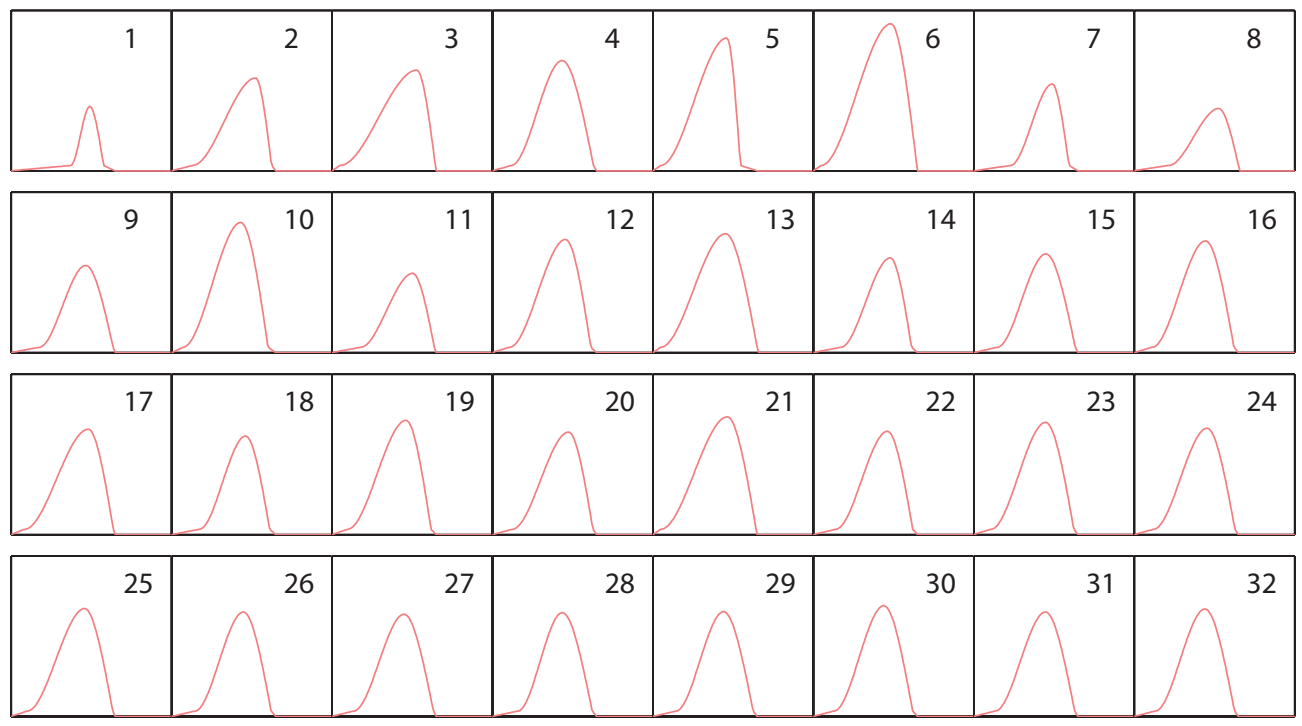

Desired toruqe for current conditions

Figure 7.6: Online optimization process using Nelder-Mead method. Thirty two conditions were automatically generated and evaluated. The optimized high level condition is defined the means of the last four conditions evaluated and the new condition generated at the end of condition thirty two. 

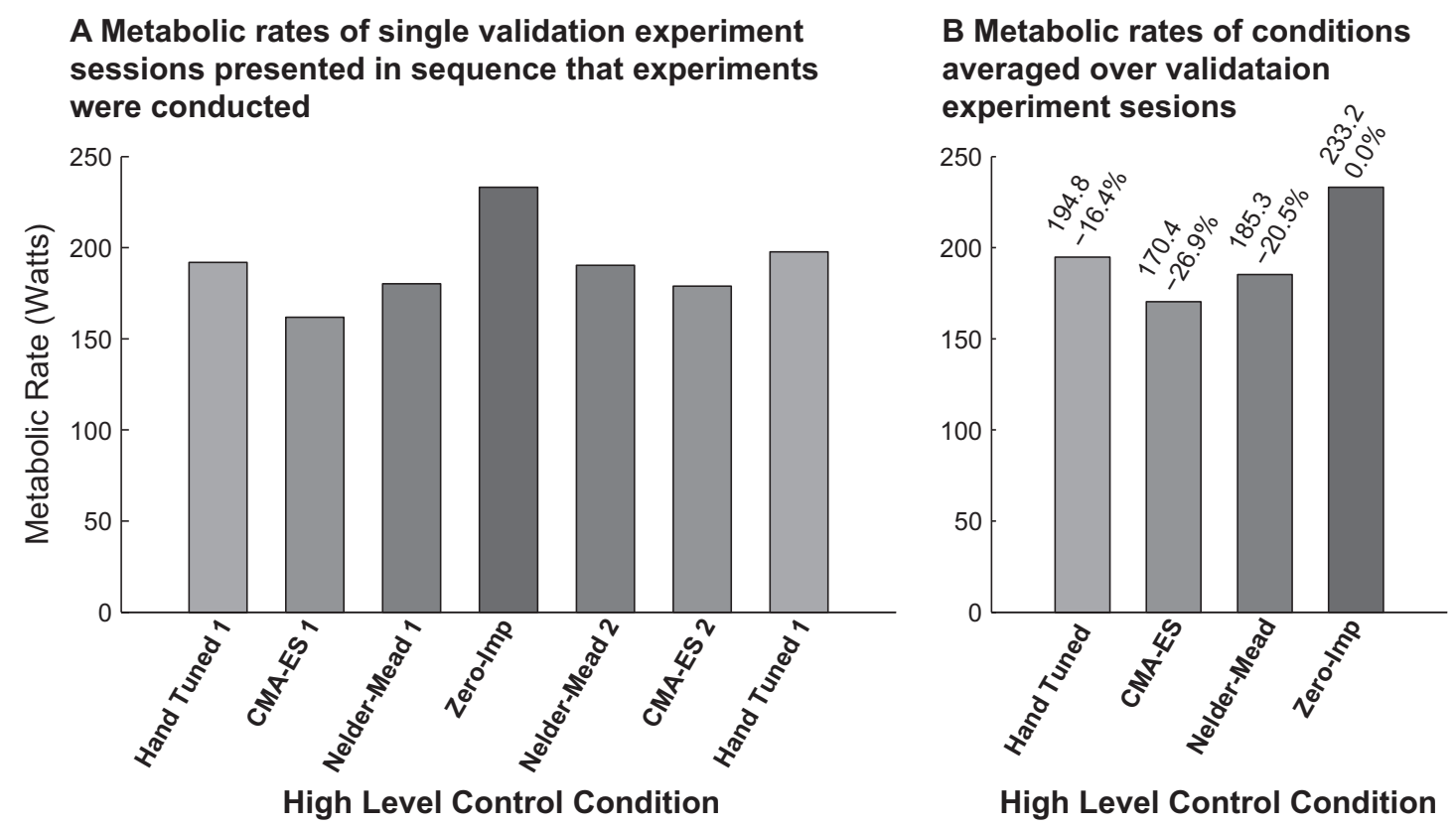

Figure 7.7: Results of online optimization validation process. A) Metabolic rates of various validation experiment sessions are presented in the sequence of test sequence. Four conditions were tested: Zero impedance mode where exoskeleton joint follows human ankle movements without exerting torques; Control condition B which was hand tuned to achieve metabolic efficiency in a prior study [172]; Optimization process identified by CMA-ES and Nelder-Mead methods. The seven experiment sessions forms two testing cycles of the four conditions to eliminate possible drifting of metabolic rates along time. For both cycles, same trends of metabolic rates were observed as zero impedance $>$ control condition B $>$ Nelder-Mead $>$ CMA-ES. B) Metabolic rates of four conditions averaged over different sessions. There is a 26.9\% drop from zero impedance condition for CMA-ES and 20.5\% for Nelder-Mead condition, both outperformed control condition B which showed 16.4\% drop of metabolic rate from the zero impedance condition. 
Due to its characteristics of being stochastic, the potentials to scale well and the option to be memoryless from generation to generation, CMA-ES was expected to work well with our application. This was confirmed by the walking experiments. However, the same Hand Tuned condition was shown to cause far more metabolic rate reduction in this study than in a prior study [172]. This means that the optimization process also realized a enforced learning and adaptation of the human body to the ankle exoskeleton. Therefore, the sucess of CMA-ES used in optimization is a combinational effect of algorithm search and human adptation.A more complete study of this approach has been conducted on multiple subjects, different exoskeletons systems and under different gait conditions, which showed signficant success. A paper based on the study has been accepted by Science [175].

Nelder-Mead, which incurs less computation power and is more straightforward to implement. Fast convergence of parameters and metabolic cost is observed. However, an occasional low output at one condition, no matter whether it is caused by a local minimum or measurement noise, will stay in the memory and result in an attraction of the final optimized value to this condition. This causes the algorithm to be not robust. Therefore, this method may result in very good or not useful results. However, it can be useful if multiple rounds of online optimization using this method are run with different initial conditions and less evaluations. This is another direction of future research. 


\section{Chapter 8}

\section{Conclusions and Future Work}

\subsection{Summary and Conclusions}

This thesis addresses the systematic control of two prominent groups of rehabilitation robotic devices: upper-limb therapeutic devices used for tracking and reaching tasks training which are made of the series connection of robot manipulators and human arms, and lower-limb exoskeletons for gait rehabilitation or human power augmentation. The aim was to identify the fundamental issues in rehabilitation robot control and to provide solutions by developing control architectures that can be generalized to multiple devices with similar traits using both theoretical and experimental methods.

In one study, a controller infrastructure for upper-limb rehabilitative robotics used in reaching and tracking training, which realizes three control modes, namely humandominant mode, robot-dominant mode and safety-stop mode, has been developed and tested through simulation and experiments. With the employment of position-dependent stiffness and position-dependent desired trajectory, the proposed controller possesses the capability of automatic smooth transition between different operation modes to re- 
alize "assist-as-needed" strategy. By this controller, the passivity of upper-limb rehabilitative robotic system has been established; the stability of the closed-loop system has also been solved with system non-linearities, uncertainties and varying human-robot interactions; the conflicts between the movements of robot and human user have also been reduced by the proposed controller in a stable manner. With this controller, the humanrobot interactive rehabilitative system moves mostly inside human-dominant region to ensure human exercise of proper amount and will be pulled back if entering robotdominant region. Built-in safety-stop functionality is realized and triggered in case of over-sized position errors to avoid damage to human limbs. While guaranteeing all the properties described, the proposed controller allows parameter adjustment, which provides the therapists with the freedom to design patient-specific training processes. Computer simulation and pilot tests on hardware have illustrated the performances of the above-mentioned capabilities of the proposed controller.

For high-energy-input low-limb rehabilitation robots used for gait training, such as powered ankle orthoses, torque control is widely used. However, due to the involvement of complicated, hard-to-model, changing human-robot interactive dynamics, and the time-sensitive characteristic of the operation, the tracking performance of the torque controllers proposed previously was far from being optimal or satisfactory in actual human walking.

Many torque control approaches have been proposed for lower-limb exoskeletons, but the relative effectiveness of various methods on the same device during human walking remains unknown, especially under different assistance methods to human, i.e., desired torque trajectories. Therefore, in another study of this thesis, I developed and compared the torque-tracking performance of nine control strategies, including variations on classical feedback control, model-based control, adaptive control and iterative learning. under realistic operating conditions was performed, and found that proportional control with damping injection compensated by iterative learning resulted 
in smaller torque errors relative to peak torque than any other approach tested or previously demonstrated. To account for interactions between patterns in desired torque and tracking performance, we tested each in combination with four high-level exoskeleton controllers that determined desired torque based on time, joint angle, a neuromuscular model, or electromyographic measurements. Controllers were implemented on an ankle exoskeleton with series elastic actuation driven by an off-board motor through a uni-directional Bowden cable. The exoskeleton was worn by one human subject walking on a treadmill at $1.25 \mathrm{~m} \cdot \mathrm{s}^{-1}$ for one hundred steady-state steps under each condition. This study found that the combination of proportional control, damping injection and iterative learning resulted in substantially lower root-mean-squared error than other torque control approaches for all high-level controllers. With time-based desired torque trajectories, rms errors were $0.6 \mathrm{Nm}$ (1.3\% of peak torque) step by step, and $0.1 \mathrm{Nm}(0.2 \%)$ on average, while with electromyography-based torque commands, errors were $2.1 \mathrm{Nm}(5.9 \%$ of peak torque) step by step and $0.2 \mathrm{Nm}(0.6 \%)$ on average. Model-free, integration-free feedback control seems to be well suited to the uncertain, changing dynamics of the human-robot system, while iterative learning is advantageous in the cyclic task of walking. Results of this study identify the best control architecture for lower-limb exoskeletons, prosthesis and even walking robots as the combination of model-free, integration-free feedback control and iterative learning.

With the proper control structure identified for torque control of lower-limb exoskeletons, additional efforts were made in this thesis to further improve the torque tracking performance of the controller by tuning control gains and hardware properties.

In one study, through mathematical analysis based on simple models of lower-limb exoskeletons, it was hypothesized that there existed an optimal iterative learning gain of motor position for real-time tracking under step-to-step gait variations after learning stabilized. This optimal gain was identified as the inverse of series elastic actuator passive stiffness. Experiments showed that for iterative learning only control, the ex- 
pected optimal gain produced lowest torque tracking errors normalized by gait variations which were represented by device joint kinematic variations. This study identified the best iterative learning gain of motor position within the best control architecture.

Another study uses theory and experiments to investigate the relationship between the passive stiffness of series elastic actuators and torque tracking performance in lower-limb exoskeletons during human walking. Through theoretical analysis with our simplified system model, it was found that the optimal passive stiffness corresponding to the slope of the desired torque-angle relationship. It was also conjectured that a bandwidth limit resulted in a maximum rate of change in torque error that can be commanded through control input, which is fixed across desired and passive stiffness conditions. This led to hypotheses about the interactions among optimal control gains, passive stiffness and desired quasi-stiffness. Walking experiments were conducted with multiple angle-based desired torque curves. The observed lowest torque tracking errors identified for each combination of desired and passive stiffnesses were shown to be linearly proportional to the magnitude of the difference between the two stiffnesses. The proportional gains corresponding to the lowest observed errors were seen inversely proportional to passive stiffness values for the same desired stiffness. These findings supported both of our hypotheses, and thus provide guidance to application-specific hardware customization as well as controller design for torque-controlled robotic legged locomotion. This study provides guidance for passive stiffness selection of lower-limb exoskeletons and other walking related robots for a fixed desired stiffness. They also provide guidance for optimal feedback gain tuning in case of changing passive or desired stiffness.

With accurate enforcement of desired assistance conditions solved by previous efforts on improving torque tracking of lower-limb exoskeleton during walking, my last study aimed to answer the question what assistance should be applied to human subjects. Due to the complicated human body inner dynamics, the optimum varies from 
person to person, and even from time to time for the same subject. The solution to these complications is an on-line optimization process based on human responses that will be conducted during the initial experimental or training session of each user. One popular human response to be investigated is the metabolic cost of human body. Previous studies by other researchers demonstrated the possibility to optimize step frequencies by metabolic cost. However, due to the essential conflicts between the long delay of human metabolic costs to actual respiratory measurements, high noise in the measurements and inability for human subjects to walk too long, the previous methods involving brutal force parameter sweeping or gradient-based optimization cannot be directly scaled to multiple parameters. In this study, through literature research and simulations, I selected two algorithms, Nelder-Mead method (NM), and Covariance Matrix Adaptation Evolution Strategy (CMA-ES), to be implemented on hardware and used to optimize exoskeleton high level controllers. In experiments, a high level controller defined as a four-parameter desired torque in time trajectory was used. Within thirty two evaluations conducted in sixty-four minutes during online optimization, both CMA-ES and Nelder-Mead showed convergence of parameters and metabolic cost. Validation process showed a $26.9 \%$ drop in metabolic cost from zero-impedance mode to the optimized condition from CMA-ES. For Nelder-Mead, the drop was 20.5\%. Both conditions outperformed an energy-efficient hand tuned condition from a prior study [172], which had a $16.4 \%$ drop from zero impedance mode. Although taking long time in optimization, CMA-ES is more robust and preferred due to its insensitivity to measurement noise and the ability to escape local minima. This study developed an integrated system to on-line optimize multiple-parameter exoskeleton high level controller using metabolic costs. The same system can be used on other devices involving human-robot interactions. This system makes it possible to easily conduct an optimization of energyefficient robot assistance for each subject at the initial session of every long rehabilitation or experiential period, results of which will improve significance of experimentbased studies and effectiveness of rehabilitation training. 
This thesis investigates the general and basic issues in control of upper-limb rehabilitation robots and lower-limb exoskeletons and provides some systematic solutions, which can be generalized to other human-robot interactive robotic devices and robot locomotive systems. The control architectures developed in this thesis provide base and starting points for researchers and developers working on similar devices.

\subsection{Future Work}

In the future, both torque control in robotic legged locomotion and high level control optimization of human-robot interactive systems have potentials to be improve.

For torque tracking, current methodologies are highly limited by our understanding of human body. Better predictions of human reactions to applied torque might leads to benefits from model-based control elements. More robust mechanical human robot interfaces can also reduce the complexity in control.

Although studies presented in this thesis and a later publication [175] have proved the effectiveness of human-in-the-loop optimization of high level controllers in humanrobot interaction systems, this topic remain a vast research area. First, although 64 min of walking experiment is fairly short for optimizing lower-lime exoskeleton high level controllers considering the high noise and slow dynamics of human response measurements, it is still exhausting for human body. CMA-ES is expected to scale well, but the total number of evaluation needed for optimization and parameter dimension still have a montonically increasing relationship. Thus, there is always motivation to reduce the total time needed for optimization, which is especially crucial when parameter dimension increases. There are several ways to address this issue. For example, certain combination of memory-based an momeoryless optimization algorithms might reduce the length of evaluation time. User preference could added as a secondary output to op- 
timization to help eliminating invalid sample conditions generated. The evaluation time for each single condition also needs to be balanced between metabolic rate estimation accuracy of single conditions and the total time needed for optimization to converge. This is a sub-optimization problem that requires modeling of the relationship between evaluation time and estimation error, simulation of human body and optimization process, and expreriments to solve.

Second, the limits of the method we used, CMA-ES, on human-robot interaction system high level controllers need to be addressed, for example, the maximum number of parameters this method can optimize. Similary, this problem needs the combination of theory, simulation and experiments to solve.

Third, the optimization process we used in this thesis is actually a combination effect of algorithmic search of the best assistance condition using CMA-ES and enforced learning of human body to the robot device. Therefore, it is a co-adaption process in human-robot interaction. Isolation of human learning and robot searching can be used to understand human dynamics and improve the optimization process design.

Finally, running various experiments of different asisstance conditions on a simulated human model can also help to address questions like the limits of human benefits with robot assistance, e.g., what happens if we apply torque equals to $100 \%$ human ankle torque?

These questions will be addressed in my future research. 


\section{Appendices}

\section{Appendix A. Stability and Convergence of the Passivity Based Controller}

\section{Passivity}

Substituting Eq. (4.19) into Eq. (4.18), we have the closed-loop equation:

$$
\frac{1}{K_{v}} \dot{s}+\frac{K_{1}}{K_{v}} s+K_{p} e \tau+K_{s} s+Y_{d}\left(\tau, \dot{\tau}_{r}, \ddot{\tau}_{r}, \dot{\theta}_{e}\right) \Delta \Gamma=\frac{K_{h}}{K_{v}} \tau_{h}
$$

in which $\Delta \Gamma=\Gamma-\tilde{\Gamma}$.

Let the output be $y=s$, multiplying both side of Eq. (1) by the output and then 
integrating it, we have

$$
\begin{aligned}
\int_{0}^{t} & \frac{K_{h}}{K_{v}} s(\varsigma) \tau_{h}(\varsigma) d \varsigma \\
= & \int_{0}^{t}\left[\frac{1}{K_{v}} s(\varsigma) \dot{s}(\varsigma)+\frac{K_{1}}{K_{v}} s^{2}(\varsigma)\right. \\
& +K_{p} \dot{e} \tau(\varsigma) e_{\tau}(\varsigma)+K_{p} \lambda e_{\tau}^{2}(\varsigma) \\
& \left.+K_{S} s^{2}(\varsigma)+s(\varsigma) Y_{d}(\varsigma) \Delta \Gamma(\varsigma)\right] d \varsigma \\
= & \underbrace{P(t)-P(0)}_{\text {Stored Energy Change }}+\underbrace{\int_{0}^{t} W(\varsigma) d \varsigma}_{\text {Dissipated Energy }}
\end{aligned}
$$

in which the stored system energy is

$$
P=\frac{1}{2 K_{v}} s^{2}+\frac{1}{2} K_{p} e_{\tau}^{2}+\frac{1}{2} \Delta \Gamma^{\top} L^{-1} \Delta \Gamma \geq 0
$$

and the energy dissipating rate is

$$
W=\left(\frac{K_{1}}{K_{v}}+K_{S}\right) s^{2}+K_{p} \lambda e_{\tau}^{2} \geq 0 .
$$

Since both $V$ and $W$ are non-negative, we can conclude the passivity of output $y$ for the system (1).

With no disturbance from human, i.e., $\tau_{h}=0$, we have

$$
\dot{P}=-W
$$

\section{Convergence}

To compensate for non-zero human loading, $\tau_{h} \neq 0$, an additional switching term is added to the controller

$$
V_{a}=-K_{p} e \tau-K_{s} s+Y_{d}\left(\tau, \dot{\tau}_{r}, \ddot{\tau}_{r}, \dot{\theta}_{e}\right) \tilde{\Gamma}-K_{s w} \operatorname{sign}(s)
$$


where $K_{S w}$ is the compensation gain. With Eq. (4), the closed loop system equation becomes

$$
\begin{aligned}
& \frac{1}{K_{v}} \dot{s}+\frac{K_{1}}{K_{v}} s+K_{p} e \tau+K_{s} s+Y_{d}\left(\tau, \dot{\tau}_{r}, \ddot{\tau}_{r}, \dot{\theta}_{e}\right) \Delta \Gamma \\
& -\frac{K_{h}}{K_{v}} \tau_{h}+K_{S w} \operatorname{sign}(s)=0 .
\end{aligned}
$$

The integral of the product of $y=s$ with the above equation is

$$
\begin{gathered}
\int_{0}^{t}\left[\frac{1}{K_{v}} s(\varsigma) \dot{s}(\varsigma)+\frac{K_{1}}{K_{v}} s^{2}(\varsigma)\right. \\
+K_{p} \dot{e} \tau(\varsigma) e \tau(\varsigma)+K_{p} \lambda e_{\tau}^{2}(\varsigma) \\
+K_{s} s^{2}(\varsigma)+s(\varsigma) Y_{d}(\varsigma) \Delta \Gamma(\varsigma) \\
\left.-s \frac{K_{h}}{K_{v}} \tau_{h}+s K_{s w} \operatorname{sign}(s)\right] d \varsigma \\
=\quad \underbrace{P(t)-P(0)}+\underbrace{\int_{0}^{t} W(\varsigma) d \varsigma}_{\text {Dissipated Energy }} \\
+\int_{0}^{t}\left[-s \frac{K_{h}}{K_{v}} \tau_{h}+s K_{s w} \operatorname{sign}(s)\right] d \varsigma \\
=0, \quad
\end{gathered}
$$

i.e.,

$$
\dot{P}=-W+s \frac{K_{h}}{K_{v}} \tau_{h}-s K_{s w} \operatorname{sign}(s)
$$

Note that

$$
\begin{aligned}
s \frac{K_{h}}{K_{v}} \tau_{h}-s K_{s w} \operatorname{sign}(s) & =s \frac{K_{h}}{K_{v}} \tau_{h}-K_{s w}|s| \\
& \leq \frac{K_{h}}{K_{v}}|s| k_{0}-K_{s w}|s|
\end{aligned}
$$

where $k_{0}$ is a finite non-negative number defined by the boundedness of the human loading:

$$
\left|\tau_{h}\right| \leq k_{0}
$$


By choosing a $K_{S w}$ such that

$$
K_{s w} \geq \frac{K_{h}}{K_{v}} k_{0}
$$

we have

$$
s \frac{K_{h}}{K_{v}} \tau_{h}-s K_{s w} \operatorname{sign}(s) \leq 0 .
$$

It is already established that

$$
W \geq 0,
$$

therefore, it is proved that

$$
\dot{P} \leq 0
$$

Based on the non-negativeness of $P$ and non-positiveness of $\dot{P}$, we have

$$
P(t) \leq P(0),
$$

i.e., $P(t)$ is bounded. Since $P$ is quadratic on $s, e \tau$ and $\Delta \Gamma$, these three terms are bounded.

Eq. (6) means

$$
\begin{aligned}
& P(t)-P(0) \\
& =-\int_{0}^{t} W(\varsigma) d \varsigma+\int_{0}^{t}\left[s(\varsigma) \frac{K_{h}}{K_{v}} \tau_{h}(\varsigma)-K_{s w}|s(\varsigma)|\right] d \varsigma .
\end{aligned}
$$

Combining Eq. (8) with Eq. (7) and (3), we have

$$
P(t)-P(0) \leq-\int_{0}^{t} W(\varsigma) d \varsigma \leq 0 .
$$

By definition, Eq. (3) and (9) lead to

$$
s, e_{\tau} \in L_{2}(0, \infty)
$$


Based on the definition of $s, \dot{e}_{\tau}$ is also bounded, and hence so is $\dot{\tau}$ since $\dot{\tau}_{d e s}$ is bounded. The boundedness of $\dot{e} \tau$ proves the uniform continuity of $e_{\tau}$.

Now due to the boundedness of $\dot{\tau}_{d e s}$ and $\ddot{\tau}_{d e s}, \dot{\tau}_{r}$ and $\ddot{\tau}_{r}$ are also bounded, which then lead to the boundedness of $Y_{d}\left(\tau, \dot{\tau}_{r}, \ddot{\tau}_{r}, \dot{\theta}_{e}\right)$. Based on Eq. (5), $\dot{s}$ is then bounded, which then proves the uniform continuity of $s$.

Combining the conclusions of uniform continuity, boundedness and Eq. (10), we have $[174,35]$ :

$$
\begin{aligned}
& s \rightarrow 0, \text { as } t \rightarrow \infty, \\
& e_{\tau} \rightarrow 0, \text { as } t \rightarrow \infty .
\end{aligned}
$$

By the definition of $s$ and $e \tau$, we then can conclude

$$
\begin{aligned}
& \tau \rightarrow \tau_{d e s}, \text { as } t \rightarrow \infty, \\
& \dot{\tau} \rightarrow \dot{\tau}_{d e s}, \text { as } t \rightarrow \infty
\end{aligned}
$$

i.e., both the actual ankle torque and its changing rate converge to the desired values.

\section{Appendix B. Neuromuscular Reflex Model}

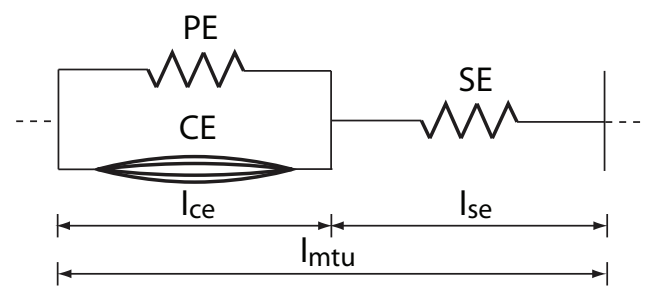

Figure 1: The Hill-type muscle-tendon-unit model includes a contractile element (CE), a parallel elastic element (PE) and series elastic element (SE).

The neuromuscular model included a Hill-type muscle-tendon-unit (MTU) (Fig. 1), with total length, $l_{m t u}$, equal to the length of the series elastic element, $l_{s e}$, plus that of the contractile element, $l_{c e}$. The length of the parallel elastic element, $l_{p e}$, was identical to $l_{c e}$. The total force produced by the MTU, $F_{m t u}$, was equal to the force on the series 
elastic element, $F_{S e}$, and also equal to the sum of the contractile element force, $F_{c e}$, and the parallel elastic element force, $F_{p e}$ (which was usually zero). The contractile element force was expressed as:

$$
F_{c e}=f_{L}\left(l_{c e}\right) \cdot f_{V}\left(i_{c e}\right) \cdot A c t \cdot F_{\max }
$$

in which $f_{L}\left(l_{c e}\right)$ and $f_{V}\left(i_{c e}\right)$ are force scaling factors that reflect the force-length and force-velocity properties of muscle, Act is the muscle activation state, and $F_{\max }$ is the maximum force that can be produced by the muscle. The force-length and forcevelocity relationships were:

$$
f_{L}\left(l_{c e}\right)=\exp \left[-\frac{\left(l_{c e}-l_{c e, o p t}\right)^{2}}{\left(W \cdot l_{c e, o p t}\right)^{2}}\right]
$$

and

$$
\begin{aligned}
& f_{V}\left(i_{c e}\right)= \\
& \begin{cases}A \frac{v_{\max }+F_{v}^{\text {max }} \cdot i_{c e}}{v_{\max } \cdot A+\dot{l}_{c e}}, & i_{c e}<0 \\
\frac{v_{\max } \cdot A+F_{v}^{\max } \cdot(A+1) \cdot\left(F_{v}^{\max }-1\right)}{v_{\max } \cdot A+i_{c e} \cdot(A+1) \cdot\left(F_{v}^{\text {max }}-1\right)}, & i_{c e} \geq 0 .\end{cases}
\end{aligned}
$$

in which $l_{c e, o p t}$ is the optimal contractile element length, $W$ is a force-length constant, $i_{c e}$ is the contractile element velocity, $A$ is a force-velocity constant, $v_{\max }$ is the maximum contraction velocity, and $F_{v}^{\max }$ is the maximum eccentric (lengthening) muscle scaling factor.

Series and parallel elastic element forces were determined as:

$$
F_{s e}=F_{\max } \cdot \frac{1}{\left(u_{\max } \cdot l_{s e, s l}\right)^{2}} \cdot \max \left(\delta l_{s e}, 0\right)^{2}
$$


and

$$
F_{p e}= \begin{cases}k_{1} \cdot \delta l_{p e}+F_{\max } \cdot \frac{k_{p e}}{l_{c e, o p t}^{2}} \cdot \delta l_{p e}^{2}, & \delta l_{p e}>0, \\ k_{1} \cdot \delta l_{p e}, & \delta l_{p e} \leq 0 .\end{cases}
$$

where $\delta l_{p e}=l_{c e}-1.5 \cdot l_{c e, o p t}$ and $\delta l_{s e}=l_{s e}-l_{s e, s l}$, and $l_{s e, s l}$ is the slack length of the series elastic element, $u_{\max }$ is an elastic element curve parameter and $k_{1}$ is the sub-slack elastic element stiffness of the parallel elastic element.

The virtual muscle had activation dynamics as:

$$
\begin{aligned}
& A c t(t=0)=\text { PreAct } \\
& \dot{A c t}=(\text { Stim }- \text { Act }) \cdot\left(\frac{\text { Stim }}{t_{a}}-\frac{1-\text { Stim }}{t_{d}}\right)
\end{aligned}
$$

where Stim is neural stimulation from the positive reflex mechanism of the virtual neural system, $t_{a}$ and $t_{d}$ are muscle activation and deactivation time constants respectively, and the initial activation value $A(t=0)$ is defined by PreAct.

The parameter values used in this study are listed in Table 1.

Table 1: Muscle model parameter values used in NMM

\begin{tabular}{llll}
\hline Param & Value & Param & Value \\
\hline$l_{c e, o p t}$ & $0.055 \mathrm{~m}$ & $l_{\text {se }, s l}$ & $0.245 \mathrm{~m}$ \\
$l_{\text {mtu }, 0}$ & $0.284 \mathrm{~m}$ & $F_{\max }$ & $1000 \mathrm{~N}$ \\
$A$ & 0.25 & $W$ & 0.56 \\
$F_{v, \max }$ & 1.8 & $u_{\max }$ & 0.04 \\
$v_{\max }$ & $0.55 \mathrm{~m} / \mathrm{s}$ & $k_{1}$ & 1 \\
$t_{a}$ & $0.02 \mathrm{~s}$ & $t_{d}$ & $0.05 \mathrm{~s}$ \\
PreAct & 0.05 & & \\
\hline
\end{tabular}




\section{Appendix C. $\mathbf{P D}^{*}+\Delta \mathrm{LRN}$ versus $\mathrm{LRN}+\mathbf{P D}^{*}$}

For controller $L^{9}$, i.e., $\mathrm{PD}^{*}+\Delta \mathrm{LRN}$, as described in Eq. (4.27), the total desired motor displacement at a certain time stamp $t$ within step $n$, with a forgetting factor $\beta=1$ and a filtering factor $\mu=1$, is

$$
\begin{aligned}
&\left.\Delta \theta_{p, \text { des }}(n)\right|_{t}= \underbrace{\left.\Delta \theta_{p, d e s}^{L R N}(0)\right|_{t}-\left.K_{l} \sum_{m=1}^{n-1} e_{\tau}(m)\right|_{t}}_{\text {Step-wise Integral Control }} \\
& \underbrace{-\left.K_{p} e \tau(n)\right|_{t}}_{\text {Proportional Control Damping Injection }} \underbrace{-\left.K_{d} \dot{\theta}_{p}(n)\right|_{t}}
\end{aligned}
$$

in which the operator

$$
\left.*(n)\right|_{t}
$$

denotes the variable $*$ at time $t$ within step $n$, i.e., time lapsed from the latest heel strike. The term

$$
\left.\Delta \theta_{p, d e s}^{L R N}(0)\right|_{t}
$$

denotes the initial value of the desired motor displacement to be learned. Iterative learning of desired motor displacement realizes a step-wise integral control action. Considering Eq. (11), it can be seen that $L^{9}$ is analogous to traditional PID control; damping injection improves system stability in the manner of derivative control, and iterative learning takes eliminates steady-state errors across steps similar to integral control. 


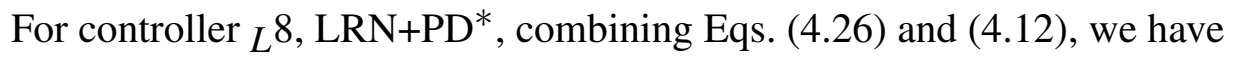

$$
\begin{aligned}
\left.\dot{\theta}_{p, d e s}(n)\right|_{t}= & \left.\frac{1}{T} \Delta \theta_{p, d e s}(n)\right|_{t} \\
= & \frac{1}{T}\left[\left.\theta_{p, d e s}^{L R N}(0)\right|_{t}-\left.K_{l} \sum_{m=1}^{n-1} e \tau(m)\right|_{t}-\left.\theta_{p}(n)\right|_{t}\right. \\
& \left.-\left.K_{p} e \tau(n)\right|_{t}-\left.K_{d} \dot{\theta}_{p}(n)\right|_{t}\right]
\end{aligned}
$$

when $\beta=1$ and $\mu=1$.

Assuming perfect motor velocity tracking:

$$
\left.\dot{\theta}_{p}(n)\right|_{t}=\left.\dot{\theta}_{p, d e s}(n)\right|_{t}
$$

then Eq. (4.26) becomes

$$
\begin{aligned}
\left.\dot{\theta}_{p}(n)\right|_{t}= & \frac{1}{T}\left[\left.\theta_{p, d e s}^{L R N}(0)\right|_{t}-\left.K_{l} \sum_{m=1}^{n-1} e_{\tau}(m)\right|_{t}-\left.\theta_{p}(n)\right|_{t}\right. \\
& \left.-\left.K_{p} e_{\tau}(n)\right|_{t}-\left.K_{d} \dot{\theta}_{p}(n)\right|_{t}\right]
\end{aligned}
$$

which can be written as

$$
\begin{aligned}
\left.\dot{\theta}_{p}(n)\right|_{t}= & -\left.\frac{1}{T+K_{d}} \theta_{p}(n)\right|_{t} \\
& +\frac{1}{T+K_{d}}\left[\left.\theta_{p, \text { des }}^{L R N}(0)\right|_{t}-\left.K_{l} \sum_{m=1}^{n-1} e_{\tau}(m)\right|_{t}\right. \\
& \left.-\left.K_{p} e_{\tau}(n)\right|_{t}\right]
\end{aligned}
$$

Assuming the same ankle kinematics for each step and the same motor position at each heel strike:

$$
\left.\theta_{p}(n)\right|_{0}=\left.\theta_{p}\right|_{0},
$$

the dynamics described in Eq. (15) can be treated as a linear-time-invariant system 
starting from the latest heel strike in the format of

$$
\dot{x}=A x+B u
$$

where $x$ is $\theta_{p}(n), A$ is $-\frac{1}{T+K_{d}}$, and $B u$ is

$$
\frac{1}{T+K_{d}}\left[\left.\theta_{p, d e s}^{L R N}(0)\right|_{t}-\left.K_{l} \sum_{m=1}^{n-1} e_{\tau}(m)\right|_{t}-\left.K_{p} e_{\tau}(n)\right|_{t}\right]
$$

Therefore, the solution of LTI system as described in Eq. (15) is

$$
\begin{aligned}
\left.\theta_{p}(n)\right|_{t}= & \left.\theta_{p}\right|_{0} \exp \left(-\frac{t}{T+K_{d}}\right) \\
& +\frac{1}{T+K_{d}} \int_{0}^{t} \exp \left(-\frac{t-\varsigma}{T+K_{d}}\right)\left[\left.\theta_{p, d e s}^{L R N}(0)\right|_{t}\right. \\
& \left.-\left.K_{l} \sum_{m=1}^{n-1} e_{\tau}(m)\right|_{\varsigma}-\left.K_{p} e_{\tau}(n)\right|_{\varsigma}\right] d \varsigma
\end{aligned}
$$

in which $t$ denotes the time lapsed since the last heel strike and $\varsigma \in[0, t]$ is a variable tracing $t$.

Therefore, the control input of $L^{8}$ can be expressed as in Eq. (18). It can be seen that the $\mathrm{LRN}+\mathrm{PD}^{*}$ controller differs from the $\mathrm{PD}^{*}+\Delta \mathrm{LRN}$ controller in that it has additional exponential and low-pass filtered continuous-time integral terms. These arise from the 
presence of $-\theta_{p}(n)$ in the control input.

$$
\begin{aligned}
\left.\Delta \theta_{p, \text { des }}(n)\right|_{t}= & \underbrace{\left.\theta_{p, d e s}^{L R N}(0)\right|_{t}-\left.K_{l} \sum_{m=1}^{n-1} e_{\tau}(m)\right|_{t}}_{\text {Step-wise Integral Control }} \underbrace{-\left.K_{p} e_{\tau}(n)\right|_{t}}_{\text {Proportional Control Damping Injection }} \underbrace{-\left.K_{d} \dot{\theta}_{p}(n)\right|_{t}} \\
& -\left.\theta_{p}\right|_{0} \exp \left(-\frac{t}{T+K_{d}}\right)-\frac{1}{T+K_{d}} \int_{0}^{t} \exp \left(-\frac{t-\varsigma}{T+K_{d}}\right)\left[\theta_{p, d e s}^{L R N}(0)-\left.K_{l} \sum_{m=1}^{n-1} e_{\tau}(m)\right|_{\zeta}-\left.K_{p} e_{\tau}(n)\right|_{\zeta}\right] d \varsigma
\end{aligned}
$$

This difference can also be illustrated in the frequency domain. The convolution term

$$
\begin{array}{r}
\int_{0}^{t} \exp \left(-\frac{t-\varsigma}{T+K_{d}}\right)\left[\left.\theta_{p, d e s}^{L R N}(0)\right|_{t}\right. \\
\left.-\left.K_{l} \sum_{m=1}^{n-1} e_{\tau}(m)\right|_{\varsigma}-\left.K_{p} e \tau(n)\right|_{\varsigma}\right] d \varsigma
\end{array}
$$

translates to

$$
\frac{1}{s+\frac{1}{T+K_{d}}}\left[\left.\theta_{p, d e s}^{L R N}(0)\right|_{s}-\left.K_{l} \sum_{m=1}^{n-1} e_{\tau}(m)\right|_{s}-\left.K_{p} e_{\tau}(n)\right|_{s}\right]
$$

in frequency domain. The low-pass filter term

$$
\frac{1}{s+\frac{1}{T+K_{d}}}
$$

realizes constant scaling at low frequency and integration at high frequency. Each evaluation of the term to be filtered,

$$
\left.\theta_{p, d e s}^{L R N}(0)\right|_{t}-\left.K_{l} \sum_{m=1}^{n-1} e_{\tau}(m)\right|_{t}-\left.K_{p} e \tau(n)\right|_{t}
$$


is generated independently at the sampling frequency. Therefore, the term is expected to have high frequency. A continuous-time integration effect is therefore included in the control input with the $\mathrm{LRN}+\mathrm{PD}^{*}$ controller.

In our experimental comparison, we found that continuous-time integral action is not desirable due to the linear accumulation of terms that are not linearly correlated. Therefore, $\mathrm{PD}^{*}+\Delta \mathrm{LRN}$ is preferred due to its simplicity and lack of continuous-time integration. 


\section{Publications}

\section{Journal Articles}

1) J. Zhang, P. Fiers, K. A. Witte, R. W. Jackson, K. L. Poggensee, C. G. Atkeson and S. H. Collins, "Human-in-the-loop optimization of exoskeleton assistance during walking”, Science, accepted.

2) J. Zhang and S. H. Collins, "The passive series stiffness that optimizes torque tracking for a lower-limb exoskeleton in human walking”, Frontiers in Neurorobotics, in revision.

3) J. Zhang, and S. H. Collins, "The iterative learning gain that optimizes real-time torque tracking after stabilization for lower-limb exoskeleton during walking.", In preparation for submission to IEEE Robotics and Automation Letters (R-AL).

4) J. Zhang and C. C. Cheah, "Passivity and Stability of Human-Robot Interaction Control for Upper-limb Rehabilitation Robot.” IEEE Transactions of Robotics, vol. 31, issue 2. 2015. 


\section{Book Chapters}

1) J. Zhang, C. C. Cheah, and S. H. Collins, "Chapter 5: Torque control in legged locomotion.", in book Bioinspired Legged Locomotion, Editor: Andre Seyfarth, Maziar Ahmad Sharbafi. Elsevier. In press.

\section{Peer-reviewed Conference Proceedings}

1) J. Zhang, C. C. Cheah, and S. H. Collins, "Experimental comparison of torque control methods on an ankle exoskeleton during human walking.", 2015 IEEE International Conference of Robotics and Automation (ICRA), 2015.

2) K. A. Witte, J. Zhang, R. W. Jackson, and S. H. Collins, "Design of Two Lightweight, High Bandwidth Torque-Controlled Ankle Exoskeletons.”, 2015 IEEE International Conference of Robotics and Automation (ICRA), 2015.

3) J. Zhang, C. C. Cheah, and S. H. Collins, "Stable human-robot interaction control for upper-limb rehabilitation robotics." in Proceedings of IEEE International Conference of Robotics and Automation (ICRA). Munich, Germany, 2013. 


\section{Bibliography}

[1] H. I. Krebs, N. Hogan, M. L. Aisen, and B. T. Volpe, "Robot-aided neurorehabilitation," IEEE transactions on rehabilitation engineering, vol. 6, pp. 75-87, Mar. 1998.

[2] G. L. Cobb, "Walking motion,” U.S. Patent 2010 482, 1945.

[3] P. Filippi, "Devide for the automatic control of the articulation of the knee applicable to a prosthesis of the thigh," U.S. Patent 2305 291, 1942.

[4] N. Yagn, "Apparatus for facilitating walking, running, and jumping," U.S. Patents 420179 and 438 830, 1890.

[5] N. Hogan, H. I. Krebs, J. Charnnarong, P. Srikrishna, and A. Sharon, "Mitmanus: A workstation for manual therapy and training," in Proceedings of International Workshop on Robot Human Communication, Sep. 1992, pp. 161-165.

[6] G. S. Sawicki and D. P. Ferris, "Powered ankle exoskeletons reveal the metabolic cost of plantar flexor mechanical work during walking with longer steps at constant step frequency," Journal of Experimental Biology, vol. 212, no. 1, pp. 2131, 2009.

[7] K. A. Witte, J. Zhang, R. W. Jackson, and S. H. Collins, "Design of two lightweight, high-bandwidth torque-controlled ankle exoskeletons," in Proceedings of the IEEE International Conference on Robotics and Automation (ICRA), Seattle, WA, 2015.

[8] T. McGeer, "Principles of walking and running," Advances in Comparative and Environmental Physiology, 1992.

[9] D. A. Rosenbaum, Human motor control. Academic press, 2009.

[10] S. Freud, Project for a scientific psychology, 1895.

[11] D.O.Hebb, The organization of behavior. New York: Willey \& Sons, 1949.

[12] C. Shatz, “The developing brain,” Scientific American, vol. 267, pp. 60-67, 1992.

[13] B. B. Johansson, "Brain plasticity and stroke rehabilitation the willis lecture," Stroke, vol. 31, no. 1, pp. 223-230, 2000. 
[14] D. J. Reinkensmeyer, L. E. Kahn, M. Averbuch, A. McKenna-Cole et al., "Understanding and treating arm movement impairment after chronic brain injury: progress with the arm guide," Journal of rehabilitation research and development, vol. 37, no. 6, p. 653, 2000.

[15] C. G. Burgar, P. S. Lum, P. C. Shor, and H. M. Van der Loos, "Development of robots for rehabilitation therapy: the palo alto va/stanford experience," Journal of rehabilitation research and development, vol. 37, no. 6, pp. 663-674, 2000.

[16] J. J. Daly, N. Hogan, E. M. Perepezko, H. I. Krebs et al., "Response to upperlimb robotics and functional neuromuscular stimulation following stroke," Journal of rehabilitation research and development, vol. 42, no. 6, p. 723, 2005.

[17] J. Basmajian, C. Gowland, M. Finlayson, A. Hall, L. Swanson, P. Stratford, J. Trotter, and M. Brandstater, "Stroke treatment: comparison of integrated behavioral-physical therapy vs traditional physical therapy programs." Archives of Physical Medicine and Rehabilitation, vol. 68, no. 5 Pt 1, pp. 267-272, 1987.

[18] D. A. Winter and D. Robertson, "Joit torque and energy patterns in normal gait," Biological cybernetics, vol. 29, no. 3, pp. 137-142, 1978.

[19] D. A. Winter, The biomechanics and motor control of human gait: normal, elderly and pathological, 2nd ed. Waterloo: University of Waterloo Press, 1991.

[20] D. P. Ferris, K. E. Gordon, G. S. Sawicki, and A. Peethambaran, "An improved powered ankle-foot orthosis using proportional myoelectric control," Gait \& posture, vol. 23, no. 4, pp. 425-428, 2006.

[21] N. Hogan and S. P. Buerger, "Impedance and interaction control," in Robotics and Automation Handbook. New York: CRC Press, 2005, pp. 1901-1924.

[22] J. R. Andres and N. Hogan, "Impedance cotrol as a framework for implementing obstacle avoidance in a manipulator," Control of Manufacturing Processes and Robotic Systems, pp. 243-251, 1983.

[23] N. Hogan, "Impedance control: an approach to manipulation," ASME Journal of Dynamic Systems, Measurement, and Control, vol. 107, no. 1, Mar. 1985.

[24] L. Marchal-Crespo and D. J. Reinkensmeyer, "Review of control strategies for robotic movement training after neurologic injury," Journal of NeuroEngineering and Rehabilitation, vol. 6, no. 20, Jun. 2009.

[25] A. de Luca, A. Albu-Schaffer, S. Haddadin, and G. Hirzinger, "Collision detection and safe reaction with the DLR-III lightweight manipulator arm," in Proceedings of the IEEE/RSJ International Conference on Intelligent Robots and Systems (IROS). IEEE, 2006, pp. 1623-1630.

[26] S. Haddadin, A. Albu-Schaffer, A. De Luca, and G. Hirzinger, "Collision detection and reaction: A contribution to safe physical human-robot interaction," in Proceedings of the IEEE/RSJ International Conference on Intelligent Robots and Systems (IROS). IEEE, 2008, pp. 3356-3363. 
[27] P. A. Lasota, G. F. Rossano, and J. A. Shah, "Toward safe close-proximity human-robot interaction with standard industrial robots," in Automation Science and Engineering (CASE), 2014 IEEE International Conference on. IEEE, 2014, pp. 339-344.

[28] J. F. Veneman, R. Kruidhof, E. E. Hekman, R. Ekkelenkamp, E. H. Van Asseldonk, and H. Van Der Kooij, "Design and evaluation of the lopes exoskeleton robot for interactive gait rehabilitation," Neural Systems and Rehabilitation Engineering, IEEE Transactions on, vol. 15, no. 3, pp. 379-386, 2007.

[29] A. H. A. Stienen, E. E. G. Hekman, H. ter Braak, A. M. M. Aalsma, F. C. T. van der Helm, and H. van der Kooij, "Design of a rotational hydroelastic actuator for a powered exoskeleton for upper limb rehabilitation," IEEE Transactions on Biomedical Engineering, vol. 57, pp. 728-735, 2010.

[30] P. Malcolm, W. Derave, S. Galle, and D. De Clercq, "A simple exoskeleton that assists plantarflexion can reduce the metabolic cost of human walking," PloS one, vol. 8, no. 2, p. e56137, 2013.

[31] G. A. Pratt and M. M. Williamson, "Series elastic actuators," in Proceedings of the IEEE/RSJ International Conference on Intelligent Robots and Systems (IROS), vol. 1. IEEE, 1995, pp. 399-406.

[32] M. Zinn, O. Khatib, B. Roth, and J. K. Salisbury, "Playing it safe [humanfriendly robots]," IEEE Robotics \& Automation Magazine, vol. 11, no. 2, pp. 12-21, 2004.

[33] D. W. Robinson, J. E. Pratt, D. J. Paluska, and G. A. Pratt, "Series elastic actuator development for a biomimetic walking robot," in Advanced Intelligent Mechatronics, 1999. Proceedings. 1999 IEEE/ASME International Conference on. IEEE, 1999, pp. 561-568.

[34] J.-J. E. Slotine and W. Li, Applied Nonlinear Control. Prentice-Hall International, Inc, 1991.

[35] S. Arimoto, Control Theory of Non-linear Mechanical Systems: A Passivitybased and Circuit-theoretic Approach. Oxford University Press, Dec. 1996.

[36] I. M. R. Ortega, A. J. Schaft and B. Maschke, "Putting energy back in control," IEEE Control Systems Magazine, pp. 0272-1708, 2001.

[37] S. Arimoto, S. Kawamura, and F. Miyazaki, "Bettering operation of robots by learning," Journal of Robotic systems, vol. 1, no. 2, pp. 123-140, 1984.

[38] N. Hansen, A. Ostermeier, and A. Gawelczyk, "On the adaptation of arbitrary normal mutation distributions in evolution strategies: The generating set adaptation." in ICGA. Citeseer, 1995, pp. 57-64.

[39] N. Hansen, "The cma evolution strategy: a comparing review," in Towards a new evolutionary computation. Springer, 2006, pp. 75-102. 
[40] J. Zhang, C. C. Cheah, and S. H. Collins, "Stable human-robot interaction control for upper-limb rehabilitation robotics," in Proceedings of IEEE International Conference on Robotics and Automation, Karlsruhe, Germany, May 2013, pp. 2201-2206.

[41] J. Zhang and C. C. Cheah, "Passivity and stability of human-robot interaction control for upper-limb rehabilitation robots," IEEE Transactions on Robotics, vol. 31, pp. 233-245, 2015.

[42] M. Hallett, "Plasticity in the human motor system," Neuroscientist, vol. 5, pp. 324-332, Sep. 1999.

[43] W. M. Jenkins and M. M. Merzenich, "Reorganization of neurocortical representations after brain injury: A neurophysiological model of the bases of recovery from stroke," Progress in Brain Research, pp. 249-266, 1987.

[44] M. Takegaki and S. Arimoto, "A new feedback method for dynamic control of manipulators," Journal of Dynamic Systems, Measurement, and Control, vol. 103, pp. 119-125, 1981.

[45] J. J. E. Slotine and W. Li, "On the adaptive control of robot manupulators," International Journal of Robotics Research, vol. 6, no. 3, pp. 45-59, 1987.

[46] C. C. Cheah, M. Hirano, S. Kawamura, and S. Arimoto, "Approximate jacobian control for robots with uncertain kinematics and dynamics," IEEE Transactions on Robotics and Automation, vol. 19, no. 4, pp. 692-702, Aug. 2003.

[47] H. Kazerooni, J.-L. Racine, L. Huang, and R. Steger, "On the control of the berkeley lower extremity exoskeleton (bleex)," in Proceedings of IEEE International Conference of Robotics and Automation, Barcelona, Spain, Apr. 2005, pp. 4353-4360.

[48] J. A. Saglia, N. G. Tsagarakis, J. S. Dai, and D. G. Caldwell, "Control strategies for ankle rehabilitation using a high performance ankle exerciser," in Proceedings of IEEE International Conference on Robotics and Automation, May 2010, pp. 2221-2227.

[49] E. D. Fasse and N. Hogan, "Control of physical contact and dynamic interaction," in Proceedings of the 7th International Symposium on Robotics Research, Munich, 1996, pp. 28-38.

[50] T. Nef, M. Mihelj, and R. Riener, "Armin: a robot for patient-cooperative arm therapy," Medical and Biological Engineering and Computing, pp. 887-900, 2007.

[51] S. K. Banala, S. K. Agrawal, and J. P. Scholz, "Active leg exoskeleton (alex) for gait rehabilitation of motor-impaired patients," in Proceedings of IEEE 10th International Conference on Rehabiliatation Robotics, 2007, pp. 401-407. 
[52] L. L. Cai, A. J. Fong, C. K. Otoshi, Y. Liang, J. W. Burdick, R. R. Roy, and V. R. Edgerton, "Implications of assist-as-needed robotic step training after a complete spinal cord injury on intrinsic strategies of motor learning," Journal of Neuroscience, vol. 26, no. 41, pp. 10654-10 658, 2006.

[53] N. Vitiello, T. Lenzi, S. Roccella, S. M. M. D. Rossi, E. Cattin, F. Vecchi, and M. C. Carrozza, "Neuroexos: a powered elbow exoskeleton for physical rehabilitation," IEEE Transactions on Robotics, vol. 29, pp. 220-235, 2013.

[54] G. A. Ollinger, J. Colgate, M. A. Peshkin, and A. Goswami, "Inertia compensation control of a one-degree-of-freedom exoskeleton for lower-limb assistance: initial experiments," IEEE Transactions on Neural Systems and Rehabilitation Engineering, vol. 20, pp. 68-77, 2012.

[55] S. Haddadin, A. Albu-Schäffer, and G. Hirzinger, "Safety analysis for a humanfriendly manipulator," International Journal of Social Robotics, vol. 2, pp. 235$252,2010$.

[56] R. Riener, L. Lunenburger, S. Jezernik, J. M. Anderschitz, G. Colombo, and V. Dietz, "Patient-cooperative strategies for robot-aided treadmill training: first experimental results," IEEE Transactions of Neural Systems and Rehabilitation Engineering, vol. 13, no. 3, pp. 380-394, 2005.

[57] S. Hanneton, A. Berthoz, J. Droulez, and J. J. E. Slotine, "Does the brain use sliding variables for the control of movements?" Biological Cybernetics, vol. 77, pp. 381-393, 1997.

[58] M. A. Finley, S. E. Fasoli, L. Dipietro, J. Ohlhoff, L. MacClellan, C. Meister, J. Whitall, R. Macko, C. T. Bever, H. I. Krebs, and N. Hogan, "Short-duration robotic therapy in stroke patients with severe upper-limb motor impairment," Journal of Rehabilitation Research \& Development, vol. 42, no. 5, pp. 683-692, 2005.

[59] S. Masiero, M. Armani, and G. Rosati, "Upper-limb robot-assisted therapy in rehabilitation of acute stroke patients: Focused review and results of new randomized controlled trial," Journal of Rehabilitation Research \& Development, vol. 48, no. 4, pp. 355-366, 2011.

[60] J. Zhang, C. C. Cheah, and S. H. Collins, "Experimental comparison of torque control methods on an ankle exoskeleton during human walking," in Proceedings of the IEEE International Conference on Robotics and Automation (ICRA), Seattle, WA, 2015.

[61] J. Zhang, C. C. Cheah, and S. H. Colins, "Torque control in legged locomotion," in Bio-inspired Legged Locomotion, M. A. Sharbafi and A. Seyfarth, Eds. Elsevier, 2016, in process.

[62] W. Cloud, "Man amplifiers: Machines that let you carry a ton," Popular Science, vol. 187, no. 5, pp. 70-73, 1965. 
[63] R. S. Mosher, "Handyman to hardiman," SAE Technical Paper, Tech. Rep., 1967.

[64] G. Schmeissser and W. Seamone, "An upper limb prosthesis-orthosis power and control system with multi-level potential," The Journal of Bone \& Joint Surgery, vol. 55, no. 7, pp. 1493-1501, 1973.

[65] M. Vukobratovic, D. Hristic, and Z. Stojiljkovic, "Development of active anthropomorphic exoskeletons," Medical and Biological Engineering, vol. 12, no. 1, pp. 66-80, 1974.

[66] S. Jezernik, G. Colombo, T. Keller, H. Frueh, and M. Morari, "Robotic orthosis lokomat: A rehabilitation and research tool," Neuromodulation, vol. 6, no. 2, pp. 108-115, 2003.

[67] G. Aguirre-Ollinger, J. E. Colgate, M. A. Peshkin, and A. Goswami, "Activeimpedance control of a lower-limb assistive exoskeleton," in Proceedings of the IEEE International Conference on Rehabilitation Robotics (ICORR). IEEE, 2007, pp. 188-195.

[68] K. Suzuki, G. Mito, H. Kawamoto, Y. Hasegawa, and Y. Sankai, "Intentionbased walking support for paraplegia patients with robot suit HAL," Advanced Robotics, vol. 21, no. 12, pp. 1441-1469, 2007.

[69] B.-C. Tsai, W.-W. Wang, L.-C. Hsu, L.-C. Fu, and J.-S. Lai, "An articulated rehabilitation robot for upper limb physiotherapy and training," in Proceedings of the IEEE/RSJ International Conference on Intelligent Robots and Systems (IROS). IEEE, 2010, pp. 1470-1475.

[70] J. Hidler, D. Nichols, M. Pelliccio, K. Brady, D. D. Campbell, J. H. Kahn, and T. G. Hornby, "Multicenter randomized clinical trial evaluating the effectiveness of the Lokomat in subacute stroke," Neurorehabilitation \& Neural Repair, vol. 23, no. 1, pp. 5-13, 2009.

[71] S. Mochon and T. McMahon, "Ballistic walking: an improved model," Mathematical Biosciences, vol. 52, pp. 241-260, 1980.

[72] T. McGeer, "Passive dynamic walking," International Journal of Robotics Research, vol. 9, no. 2, pp. 62-82, 1990.

[73] S. Collins, A. Ruina, R. Tedrake, and M. Wisse, "Efficient bipedal robots based on passive-dynamic walkers," Science, vol. 307, no. 5712, pp. 1082-1085, 2005.

[74] B. W. Verdaasdonk, H. F. J. M. Koopman, and F. C. van der Helm, "Energy efficient walking with central pattern generators: from passive dynamic walking to biologically inspired control," Biological Cybernetics, vol. 101, pp. 49-61, 2009.

[75] A. J. Ijspeert, "Biorobotics: Using robots to emulate and investigate agile locomotion," Science, vol. 346, pp. 196-203, 2014. 
[76] J. Pratt, P. Dilworth, and G. Pratt, "Virtual model control of a bipedal walking robot," in Robotics and Automation, 1997. Proceedings., 1997 IEEE International Conference on, vol. 1. IEEE, 1997, pp. 193-198.

[77] S. Au, M. Berniker, and H. Herr, "Powered ankle-foot prosthesis to assist levelground and stair-descent gaits," Neural Networks, vol. 21, no. 4, pp. 654-666, 2008.

[78] F. Sup, H. A. Varol, J. Mitchell, T. J. Withrow, and M. Goldfarb, "Preliminary evaluations of a self-contained anthropomorphic transfemoral prosthesis," IEEE Transactions on Mechatronics, vol. 14, no. 6, pp. 667-676, 2009.

[79] J. M. Caputo and S. H. Collins, "An experimental robotic testbed for accelerated development of ankle prostheses," in Proceedings of the IEEE International Conference on Robotics and Automation (ICRA). IEEE, 2013, pp. 2645-2650.

[80] H. Kawamoto, S. Taal, H. Niniss, T. Hayashi, K. Kamibayashi, K. Eguchi, and Y. Sankai, "Voluntary motion support control of robot suit HAL triggered by bioelectrical signal for hemiplegia," in Proceedings of the IEEE International Conference of the Engineering in Medicine and Biology Society. IEEE, 2010, pp. 462-466.

[81] O. Unluhisarcikli, M. Pietrusinski, B. Weinberg, P. Bonato, and C. Mavroidis, "Design and control of a robotic lower extremity exoskeleton for gait rehabilitation," in Proceedings of the IEEE/RSJ International Conference on Intelligent Robots and Systems (IROS). IEEE, 2011, pp. 4893-4898.

[82] F. Giovacchini, F. Vannetti, M. Fantozzi, M. Cempini, M. Cortese, A. Parri, T. Yan, D. Lefeber, and N. Vitiello, "A light-weight active orthosis for hip movement assistance," Robotics and Autonomous Systems, 2014.

[83] G. A. Pratt, P. Willisson, C. Bolton, and A. Hofman, "Late motor processing in low-impedance robots: impedance control of series-elastic actuators," in Proceedings of the American Control Conference, vol. 4. IEEE, 2004, pp. 32453251.

[84] G. Wyeth, "Control issues for velocity sourced series elastic actuators," in Proceedings of the Australasian Conference on Robotics and Automation 2006. Australian Robotics and Automation Association Inc, 2006.

[85] A. Schiele, P. Letier, R. van der Linde, and F. Van der Helm, "Bowden cable actuator for force-feedback exoskeletons," in Proceedings of the IEEE/RSJ International Conference on Intelligent Robots and Systems (IROS). IEEE, 2006, pp. 3599-3604.

[86] D. Hobbelen, T. de Boer, and M. Wisse, "System overview of bipedal robots flame and tulip: Tailor-made for limit cycle walking," in Proceedings of the IEEE/RSJ International Conference on Intelligent Robots and Systems (IROS). IEEE, 2008, pp. 2486-2491. 
[87] L. M. Mooney, E. J. Rouse, and H. M. Herr, "Autonomous exoskeleton reduces metabolic cost of human walking during load carriage," Journal of NeuroEngineering and Rehabilitation, vol. 11, no. 80, pp. 0003-11, 2014.

[88] J. F. Veneman, R. Ekkelenkamp, R. Kruidhof, F. C. van der Helm, and H. van der Kooij, "A series elastic-and bowden-cable-based actuation system for use as torque actuator in exoskeleton-type robots," International Journal of Robotics Research, vol. 25, no. 3, pp. 261-281, 2006.

[89] J. M. Caputo and S. H. Collins, "A universal ankle-foot prosthesis emulator for human locomotion experiments," Journal of Biomechanical Engineering, vol. 136, no. 035002, Mar. 2014.

[90] K. Kong, J. Bae, and M. Tomizuka, "Control of rotary series elastic actuator for ideal force-mode actuation in human-robot interaction applications," IEEE Transactions on Mechatronics, vol. 14, no. 1, pp. 105-118, 2009.

[91] D. Zanotto, T. Lenzi, P. Stegall, and S. K. Agrawal, "Improving transparency of powered exoskeletons using force/torque sensors on the supporting cuffs," in Proceedings of the IEEE International Conference on Rehabilitation Robotics (ICORR). IEEE, 2013, pp. 1-6.

[92] W. van Dijk, H. Van Der Kooij, B. Koopman, and E. van Asseldonk, "Improving the transparency of a rehabilitation robot by exploiting the cyclic behaviour of walking," in Proceedings of the IEEE International Conference on Rehabilitation Robotics (ICORR). IEEE, 2013, pp. 1-8.

[93] S. H. Collins and R. W. Jackson, "Inducing self-selected human engagement in robotic locomotion training," in Proceedings of the IEEE International Conference on Rehabilitation Robotics (ICORR). IEEE, 2013, pp. 1-6.

[94] S. H. Collins, M. B. Wiggin, and G. S. Sawicki, "Reducing the energy cost of human walking using an unpowered exoskeleton," Nature, vol. 522, pp. 212$215,2015$.

[95] H. Vallery, R. Ekkelenkamp, H. Van Der Kooij, and M. Buss, "Passive and accurate torque control of series elastic actuators," in Proceedings of the IEEE/RSJ International Conference on Intelligent Robots and Systems (IROS). IEEE, 2007, pp. 3534-3538.

[96] T. Lenzi, N. Vitiello, S. M. M. De Rossi, S. Roccella, F. Vecchi, and M. C. Carrozza, "Neuroexos: a variable impedance powered elbow exoskeleton," in Proceedings of the IEEE International Conference on Robotics and Automation (ICRA). IEEE, 2011, pp. 1419-1426.

[97] N. Paine, S. Oh, and L. Sentis, "Design and control considerations for highperformance series elastic actuators," Mechatronics, IEEE/ASME Transactions on, vol. 19, no. 3, pp. 1080-1091, 2014. 
[98] A. Gupta, M. K. O’Malley, V. Patoglu, and C. Burgar, "Design, control and performance of RiceWrist: A force feedback wrist exoskeleton for rehabilitation and training," International Journal of Robotics Research, vol. 27, no. 2, pp. 233-251, 2008.

[99] R. J. Farris, H. Quintero, M. Goldfarb et al., "Preliminary evaluation of a powered lower limb orthosis to aid walking in paraplegic individuals," Neural Systems and Rehabilitation Engineering, IEEE Transactions on, vol. 19, no. 6, pp. 652-659, 2011.

[100] R. Kelly, "Regulation of manipulators in generic task space: An energy shaping plus damping injection approach," Robotics and Automation, IEEE Transactions on, vol. 15, no. 2, pp. 381-386, 1999.

[101] S. K. Banala, S. H. Kim, S. K. Agrawal, and J. P. Scholz, "Robot assisted gait training with active leg exoskeleton (ALEX)," IEEE Transactions on Neural Systems and Rehabilitation Engineering, vol. 17, no. 1, pp. 2-8, 2009.

[102] C. Fleischer, C. Reinicke, and G. Hommel, "Predicting the intended motion with EMG signals for an exoskeleton orthosis controller," in Proceedings of the IEEE/RSJ International Conference on Intelligent Robots and Systems (IROS). IEEE, 2005, pp. 2029-2034.

[103] R. Ortega and M. W. Spong, "Adaptive motion control of rigid robots: A tutorial," Automatica, vol. 25, no. 6, pp. 877-888, 1989.

[104] A. Calanca and P. Fiorini, "Human-adaptive control of series elastic actuators," Robotica, vol. 32, no. 08, pp. 1301-1316, 2014.

[105] J. Bae and M. Tomizuka, "A gait rehabilitation strategy inspired by an iterative learning algorithm," Mechatronics, vol. 22, no. 2, pp. 213-221, 2012.

[106] P. Malcolm, R. E. Quesada, J. M. Caputo, and S. H. Collins, "The influence of push-off timing in a robotic ankle-foot prostheses on the energetics and mechanics of walking," Journal of NeuroEngineering and Rehabilitation, vol. 12, no. 21, pp. 1-14, 2015.

[107] K. B. Fite and M. Goldfarb, "Design and energetic characterization of a proportional-injector monopropellant-powered actuator," IEEE Transactions on Mechatronics, vol. 11, no. 2, pp. 196-204, 2006.

[108] J. K. Hitt, T. G. Sugar, M. Holgate, and R. Bellman, "An active foot-ankle prosthesis with biomechanical energy regeneration," Journal of Medical Devices, vol. 4, no. 1, p. 011003, 2010.

[109] J. Rosen, M. Brand, M. B. Fuchs, and M. Arcan, "A myosignal-based powered exoskeleton system," Systems, Man and Cybernetics, Part A: Systems and Humans, IEEE Transactions on, vol. 31, no. 3, pp. 210-222, 2001. 
[110] E. Cavallaro, J. Rosen, J. C. Perry, S. Burns, and B. Hannaford, "Hill-based model as a myoprocessor for a neural controlled powered exoskeleton arm parameters optimization," in Proceedings of the IEEE International Conference on Robotics and Automation (ICRA), Barcelona, Spain, Apr. 2005.

[111] J. C. Perry, J. Rosen, and S. Burns, "Upper-limb powered exoskeleton design," IEEE Transactions on Mechatronics, vol. 12, no. 4, pp. 408-417, 2007.

[112] M. F. Eilenberg, H. Geyer, and H. Herr, "Control of a powered ankle-foot prosthesis based on a neuromuscular model," IEEE Transactions on Neural Systems and Rehabilitation Engineering, vol. 18, no. 2, pp. 164-173, 2010.

[113] H. Geyer and H. Herr, "A muscle-reflex model that encodes principles of legged mechanics produces human walking dynamics and muscle activities," IEEE Transactions on Neural Systems and Rehabilitation Engineering, vol. 18, no. 3, pp. 263-273, 2010.

[114] A. H. Shultz, J. E. Mitchell, D. Truex, B. E. Lawson, E. Ledoux, and M. Goldfarb, "A walking controller for a powered ankle prosthesis," in Engineering in Medicine and Biology Society (EMBC), 2014 36th Annual International Conference of the IEEE. IEEE, 2014, pp. 6203-6206.

[115] T. W. Dorn, J. M. Wang, J. L. Hicks, and S. L. Delp, "Predictive simulation generates human adaptations during loaded and inclined walking," PloS one, vol. 10, no. 4, 2015.

[116] N. Van der Noot, A. J. Ijspeert, and R. Ronsse, "Biped gait controller for large speed variations, combining reflexes and a central pattern generator in a neuromuscular model," in Proceedings of the IEEE International Conference on Robotics and Automation (ICRA). IEEE, 2015, pp. 6267-6274.

[117] J. Markowitz, P. Krishnaswamy, M. F. Eilenberg, K. Endo, C. Barnhart, and H. Herr, "Speed adaptation in a powered transtibial prosthesis controlled with a neuromuscular model," Philosophical Transactions of the Royal Society B: Biological Sciences, vol. 366, no. 1570, pp. 1621-1631, 2011.

[118] C. Loconsole, S. Dettori, A. Frisoli, C. A. Avizzano, and M. Bergamasco, "An emg-based approach for on-line predicted torque control in robotic-assisted rehabilitation," in Haptics Symposium (HAPTICS), 2014 IEEE. IEEE, 2014, pp. 181-186.

[119] S. Huang, J. P. Wensman, and D. P. Ferris, "An experimental powered lower limb prosthesis using proportional myoelectric control," Journal of Medical Devices, vol. 8, no. 2, p. 024501, 2014.

[120] K. Z. Takahashi, M. D. Lewek, and G. S. Sawicki, "A neuromechanics-based powered ankle exoskeleton to assist walking post-stroke: a feasibility study," Journal of neuroengineering and rehabilitation, vol. 12, no. 1, p. 23, 2015. 
[121] A. B. Zoss, H. Kazerooni, and A. Chu, "Biomechanical design of the berkeley lower extremity exoskeleton (bleex)," IEEE Transactions on Mechatronics, vol. 11, no. 2, pp. 128-138, 2006.

[122] S. Wang, C. Meijneke, and H. Van Der Kooij, "Modeling, design, and optimization of mindwalker series elastic joint," in Proceedings of the IEEE International Conference on Rehabilitation Robotics (ICORR). IEEE, 2013, pp. 1-8.

[123] J. S. Sulzer, R. Roiz, M. Peshkin, J. L. Patton et al., "A highly backdrivable, lightweight knee actuator for investigating gait in stroke," IEEE Transactions on Robotics, vol. 25, no. 3, pp. 539-548, 2009.

[124] T. Kobayashi, Y. Akazawa, H. Naito, M. Tanaka, S. W. Hutchins et al., "Design of an automated device to measure sagittal plane stiffness of an articulated anklefoot orthosis," Prosthetics and Orthotics International, vol. 34, no. 4, pp. 439448, 2010.

[125] D. Accoto, G. Carpino, F. Sergi, N. L. Tagliamonte, L. Zollo, and E. Guglielmelli, "Design and characterization of a novel high-power series elastic actuator for a lower limb robotic orthosis," International Journal of Advanced Robotic Systems, vol. 10, p. 359, 2013.

[126] J. Gordon and C. Ghez, "Trajectory control in targeted force impulses," Experimental brain research, vol. 67, no. 2, pp. 253-269, 1987.

[127] A. Klimchik, A. Pashkevich, D. Chablat, and G. Hovland, "Compensation of compliance errors in parallel manipulators composed of non-perfect kinematic chains," pp. 51-58, 2012.

[128] G. Heinzinger, D. Fenwick, B. Paden, and F. Miyazaki, "Stability of learning control with disturbances and uncertain initial conditions," IEEE Transactions on Automatic Control, vol. 37, no. 1, pp. 110-114, 1992.

[129] J. Van de Wijdeven, T. Donkers, and O. Bosgra, "Iterative learning control for uncertain systems: robust monotonic convergence analysis," Automatica, vol. 45, no. 10, pp. 2383-2391, 2009.

[130] E. Schuitema, M. Wisse, T. Ramakers, and P. Jonker, "The design of LEO: a 2D bipedal walking robot for online autonomous reinforcement learning," in Proceedings of the IEEE/RSJ International Conference on Intelligent Robots and Systems (IROS). IEEE, 2010, pp. 3238-3243.

[131] S. M. Cain, K. E. Gordon, and D. P. Ferris, "Locomotor adaptation to a powered ankle-foot orthosis depends on control method," Journal of NeuroEngineering and Rehabilitation, vol. 4, 2007.

[132] S. K. Au, P. Dilworth, and H. Herr, "An ankle-foot emulation system for the study of human walking biomechanics," in Proceedings of the IEEE International Conference on Robotics and Automation (ICRA). IEEE, 2006, pp. 29392945. 
[133] S. Song, J. Kim, and K. Yamane, "Development of a bipedal robot that walks like an animation character," in Proceedings of the IEEE International Conference on Robotics and Automation (ICRA), 2015, pp. 3596-3602.

[134] J. G. Ziegler and N. B. Nichols, "Optimum settings for automatic controllers," trans. ASME, vol. 64, no. 11, 1942.

[135] S. W. Sung and I.-B. Lee, "Limitations and countermeasures of pid controllers," Industrial \& engineering chemistry research, vol. 35, no. 8, pp. 2596-2610, 1996.

[136] M. Uchiyama, "Formation of high-speed motion pattern of a mechanical arm by trial," Transactions of the Society of Instrument and Control Engineers, vol. 14, pp. 706-712, 1978.

[137] M. Togai and O. Yamano, "Analysis and design of an optimal learning control scheme for industrial robots: A discrete system approach," in Decision and Control, 1985 24th IEEE Conference on. IEEE, 1985, pp. 1399-1404.

[138] M. Fukuda and S. Shin, "Model reference learning control with a wavelet network," in Iterative learning control. Springer, 1998, pp. 211-226.

[139] K. L. Moore, Iterative learning control for deterministic systems. Springer Science \& Business Media, 2012.

[140] N. Amann, D. H. Owens, and E. Rogers, "Robustness of norm-optimal iterative learning control," in Control'96, UKACC International Conference on (Conf. Publ. No. 427), vol. 2. IET, 1996, pp. 1119-1124.

[141] C. G. Atkeson and J. Mclntyre, "Robot trajecgtory learning through practice," in Proceedings of the IEEE International Conference on Robotics and Automation (ICRA), 1986, pp. 1737 - 1742.

[142] A. Hać, "Learning control in the presence of measurement noise," in American Control Conference, 1990. IEEE, 1990, pp. 2846-2851.

[143] S. S. Saab, W. G. Vogt, and M. H. Mickle, "Learning control algorithms for tracking "slowly" varying trajectories," Systems, Man, and Cybernetics, Part B: Cybernetics, IEEE Transactions on, vol. 27, no. 4, pp. 657-670, 1997.

[144] S. S. Saab, "Stochastic p-type/d-type iterative learning control algorithms," International Journal of Control, vol. 76, no. 2, pp. 139-148, 2003.

[145] _ _ "Selection of the learning gain matrix of an iterative learning control algorithm in presence of measurement noise," Automatic Control, IEEE Transactions on, vol. 50, no. 11, pp. 1761-1774, 2005.

[146] K. Salisbury, B. Eberman, M. Levin, and W. Townsend, "The design and control of an experimental whole-arm manipulator," in The fifth international symposium on Robotics research. MIT Press, 1991, pp. 233-241. 
[147] R. v. Ham, T. G. Sugar, B. Vanderborght, K. W. Hollander, and D. Lefeber, "Compliant actuator designs," Robotics \& Automation Magazine, IEEE, vol. 16, no. 3, pp. 81-94, 2009.

[148] D. E. Whitney, "Historical perspective and state of the art in robot force control," International Journal of Robotics Research, vol. 6, no. 1, pp. 3-14, 1987.

[149] J. W. Sensinger et al., "Improvements to series elastic actuators," in Proceedings of the IEEE/ASME International Conference on Mechatronic and Embedded Systems and Applications. IEEE, 2006, pp. 1-7.

[150] K. W. Hollander, R. Ilg, T. G. Sugar, and D. Herring, “An efficient robotic tendon for gait assistance," Journal of biomechanical engineering, vol. 128, no. 5, pp. 788-791, 2006.

[151] B. Vanderborght, B. Verrelst, R. Van Ham, M. Van Damme, P. Beyl, and D. Lefeber, "Development of a compliance controller to reduce energy consumption for bipedal robots," Autonomous Robots, vol. 24, no. 4, pp. 419-434, 2008.

[152] B. Vanderborght, R. Van Ham, D. Lefeber, T. G. Sugar, and K. W. Hollander, "Comparison of mechanical design and energy consumption of adaptable, passive-compliant actuators," The International Journal of Robotics Research, vol. 28 , no. 1 , pp. 90-103, 2009.

[153] B. Vanderborght, B. Verrelst, R. Van Ham, M. Van Damme, D. Lefeber, B. M. Y. Duran, and P. Beyl, "Exploiting natural dynamics to reduce energy consumption by controlling the compliance of soft actuators," The International Journal of Robotics Research, vol. 25, no. 4, pp. 343-358, 2006.

[154] A. Jafari, N. G. Tsagarakis, and D. G. Caldwell, "A novel intrinsically energy efficient actuator with adjustable stiffness (awas)," Mechatronics, IEEE/ASME Transactions on, vol. 18, no. 1, pp. 355-365, 2013.

[155] R. Ozawa, H. Kobayashi, and R. Ishibashi, "Adaptive impedance control of a variable stiffness actuator," Advanced Robotics, vol. 29, no. 4, pp. 273-286, 2015.

[156] E. J. Rouse, R. D. Gregg, L. J. Hargrove, and J. W. Sensinger, "The difference between stiffness and quasi-stiffness in the context of biomechanical modeling," Biomedical Engineering, IEEE Transactions on, vol. 60, no. 2, pp. 562-568, 2013.

[157] J. J. Collins and I. N. Stewart, "Coupled nonlinear oscillators and the symmetries of animal gaits," Journal of Nonlinear Science, vol. 3, no. 1, pp. 349-392, 1993.

[158] L. Righetti, J. Buchli, and A. J. Ijspeert, "Adaptive frequency oscillators and applications," The Open Cybernetics and Systemics Journal, vol. 3, no. BIOROBARTICLE-2010-001, pp. 64-69, 2009. 
[159] J. S. Bay and H. Hemami, "Modeling of a neural pattern generator with coupled nonlinear oscillators," Biomedical Engineering, IEEE Transactions on, no. 4, pp. 297-306, 1987.

[160] J. Collins and I. Stewart, "Hexapodal gaits and coupled nonlinear oscillator models," Biological Cybernetics, vol. 68, no. 4, pp. 287-298, 1993.

[161] J. Buchli, F. Iida, and A. J. Ijspeert, "Finding resonance: Adaptive frequency oscillators for dynamic legged locomotion," in Intelligent Robots and Systems, 2006 IEEE/RSJ International Conference on. IEEE, 2006, pp. 3903-3909.

[162] J. Morimoto, G. Endo, J. Nakanishi, S.-H. Hyon, G. Cheng, D. Bentivegna, and C. G. Atkeso, "Modulation of simple sinusoidal patterns by a coupled oscillator model for biped walking," in Robotics and Automation, 2006. ICRA 2006. Proceedings 2006 IEEE International Conference on. IEEE, 2006, pp. 1579-1584.

[163] M. S. Dutra, A. C. de Pina Filho, and V. F. Romano, "Modeling of a bipedal locomotor using coupled nonlinear oscillators of van der pol," Biological Cybernetics, vol. 88, no. 4, pp. 286-292, 2003.

[164] R. Ronsse, B. Koopman, N. Vitiello, T. Lenzi, S. M. M. De Rossi, J. van den Kieboom, E. Van Asseldonk, M. C. Carrozza, H. van der Kooij, and A. J. Ijspeert, "Oscillator-based walking assistance: A model-free approach," in Proceedings of the IEEE International Conference on Rehabilitation Robotics (ICORR). IEEE, 2011, pp. 1-6.

[165] K. Tsuchiya, S. Aoi, and K. Tsujita, "Locomotion control of a biped locomotion robot using nonlinear oscillators," in Intelligent Robots and Systems, 2003.(IROS 2003). Proceedings. 2003 IEEE/RSJ International Conference on, vol. 2. IEEE, 2003, pp. 1745-1750.

[166] S. Aoi and K. Tsuchiya, "Locomotion control of a biped robot using nonlinear oscillators," Autonomous robots, vol. 19, no. 3, pp. 219-232, 2005.

[167] W. Felt, J. C. Selinger, J. M. Donelan, and C. D. Remy, "Body-in-the-loop: Optimizing device parameters using measures of instantaneous energetic cost," PloS one, vol. 10, no. 8, p. e0135342, 2015.

[168] G. Arfken, "The method of steepest descents," Mathematical methods for physicists, vol. 3, pp. 428-436, 1985.

[169] N. V. Queipo, R. T. Haftka, W. Shyy, T. Goel, R. Vaidyanathan, and P. K. Tucker, "Surrogate-based analysis and optimization," Progress in aerospace sciences, vol. 41, no. 1, pp. 1-28, 2005.

[170] J. A. Nelder and R. Mead, "A simplex method for function minimization," The computer journal, vol. 7, no. 4, pp. 308-313, 1965.

[171] J. C. Selinger and J. M. Donelan, "Estimating instantaneous energetic cost during non-steady-state gait," Journal of Applied Physiology, pp. 1406-1415, 2014. 
[172] R. J. Jackson and S. H. Collins, "An experimental comparison of the relative benefits of work and torque assistance in ankle exoskeletons," Journal of Applied Physiology, vol. 119, pp. 541-557, 2015.

[173] J. Brockway, "Derivation of formulae used to calculate energy expenditure in man." Human nutrition. Clinical nutrition, vol. 41, no. 6, pp. 463-471, 1987.

[174] C. A. Desoer and M. Vidyasagar, Feedback systems: input-output properties. SIAM, 1975, vol. 55.

[175] J. Zhang, P. Fiers, K. A. Witte, R. W. Jackson, K. L. Poggensee, C. G. Atkeson and S. H. Collins, "Human-in-the-loop optimization of exoskeleton assistance during walking”, Science, accepted, 2017. 